\title{
Health Technology Assessment
}

Volume $25 \bullet$ Issue 48 • August 2021

ISSN 1366-5278

\section{Progressive exercise compared with best-practice advice, with or without corticosteroid injection, for rotator cuff disorders: the GRASP factorial RCT}

Sally Hopewell, David J Keene, Peter Heine, loana R Marian, Melina Dritsaki, Lucy Cureton, Susan J Dutton, Helen Dakin, Andrew Carr, Willie Hamilton, Zara Hansen, Anju Jaggi, Chris Littlewood, Karen Barker, Alastair Gray and Sarah E Lamb on behalf of the GRASP Trial Group 



\title{
Progressive exercise compared with best-practice advice, with or without corticosteroid injection, for rotator cuff disorders: the GRASP factorial RCT
}

\author{
Sally Hopewell@,$^{1 *}$ David J Keene $\odot,{ }^{2}$ Peter Heine $\odot,{ }^{2}$ \\ Ioana R Marian $\odot,{ }^{1}$ Melina Dritsaki®, ${ }^{1}$ Lucy Cureton $\odot,{ }^{2}$ \\ Susan J Dutton®, ${ }^{1}$ Helen Dakin $\odot,{ }^{3}$ Andrew Carr® ${ }^{2}$ \\ Willie Hamilton®, ${ }^{4}$ Zara Hansen $₫,{ }^{2}$ Anju Jaggi®, ${ }^{5}$ \\ Chris Littlewood®, 6 Karen Barker®, ${ }^{2}$ Alastair Gray ${ }^{3}$ \\ and Sarah E Lamb® ${ }^{2,4}$ on behalf of the GRASP Trial Group
}

${ }^{1}$ Oxford Clinical Trials Research Unit, Nuffield Department of Orthopaedics, Rheumatology and Musculoskeletal Sciences, University of Oxford, Oxford, UK ${ }^{2}$ Nuffield Department of Orthopaedics, Rheumatology and Musculoskeletal Sciences, University of Oxford, Oxford, UK

${ }^{3}$ Nuffield Department of Population Health, University of Oxford, Oxford, UK

${ }^{4}$ College of Medicine and Health, University of Exeter, Exeter, UK

${ }^{5}$ Royal National Orthopaedic Hospital NHS Trust, Stanmore, UK

${ }^{6}$ Manchester Metropolitan University, Manchester, UK

${ }^{*}$ Corresponding author

Declared competing interests of authors: Sally Hopewell is a member of the National Institute for Health Research (NIHR) Health Technology Assessment (HTA) Clinical Evaluation and Trials committee (2018 to present). David J Keene holds a NIHR Postdoctoral Fellowship (PDF-2016-09-056). Ioana R Marian holds a NIHR Pre-Doctoral Fellowship (reference NIHR300479). Helen Dakin is partly funded by a NIHR Senior Research Fellowship through the Biomedical Research Centre (Oxford, UK). Andrew Carr has a patent BioPatch issued and is a member of the UK Research and Innovation/Medical Research Council Developmental Pathway Funding Scheme Panel. He was chief investigator of the NIHR HTA UK Rotator Cuff Surgery (UKUFF) trial and the Can Shoulder Arthroscopy Work (CSAW) trial. $\mathrm{He}$ is a consultant to the Novartis Pharmaceuticals UK Ltd Musculoskeletal Board (London, UK). He is chief investigator of a NIHR Innovation for Innovation (i4i) trial of a novel electrospun patch and of a Wellcome Trust (London, UK)-funded trial of a novel electrospun suture in rotator cuff repair surgery. He was director of the NIHR Musculoskeletal British Research Unit (2008-17) and is musculoskeletal theme lead for the NIHR Oxford Biomedical Research Centre (Oxford, UK). He reports personal fees from Novartis Pharmaceuticals UK Ltd Musculoskeletal Advisory Board. Zara Hansen receives personal fees from various health-care trusts and individuals to train health-care professionals in cognitive-behavioural approaches outside the submitted work. Anju Jaggi is currently a council member of the British Shoulder \& Elbow Society (London, UK). She is co-applicant of the NIHR HTA PRO CURE (Partial Rotator Cuff Repair) trial. Chris Littlewood is chairperson of the Chartered Society of Physiotherapy Scientific Panel (London, UK). He is chief investigator of the NIHR postdoctoral fellowship-funded SPeEDy (Surgery versus PhysiothErapist-leD exercise for traumatic tears of the rotator cuff) study 

(reference PDF-2018-11-ST2-005). He was previously lead researcher for the NIHR Research for Patient Benefit-funded RaCeR (Rehabilitation following Rotator Cuff RepaiR) study (PB-PG-081620009) and chief investigator for the NIHR Doctoral Fellowship-funded SELF study (DRF-2011-04-090). Sarah E Lamb reports grants from the NIHR HTA programme during the conduct of the study and was a member of the following boards: HTA Additional Capacity Funding Board (2012-15); HTA Clinical Trials Board (2010-15); HTA End of Life Care and Add on Studies (2015); HTA Funding Boards Policy Group (formerly Clinical Studies Group) (2010-15); HTA Maternal, Newborn and Child Health Methods Group (2013-15); HTA post-board funding teleconference (2010-15); HTA Primary Care Themed Call board (2013-14); HTA Prioritisation Group (2010-15); and the NIHR Clinical Trials Unit Standing Advisory Committee (2012-16).

\section{Published August 2021}

DOI: 10.3310/hta25480

This report should be referenced as follows:

Hopewell S, Keene DJ, Heine P, Marian IR, Dritsaki M, Cureton L, et al. Progressive exercise compared with best-practice advice, with or without corticosteroid injection, for rotator cuff disorders: the GRASP factorial RCT. Health Technol Assess 2021;25(48).

Health Technology Assessment is indexed and abstracted in Index Medicus/MEDLINE, Excerpta Medica/EMBASE, Science Citation Index Expanded (SciSearch ${ }^{\circledR}$ ) and Current Contents ${ }^{\circledR} /$

Clinical Medicine. 



\title{
Health Technology Assessment
}

\author{
ISSN 1366-5278 (Print) \\ ISSN 2046-4924 (Online)
}

Impact factor: 4.014

Health Technology Assessment is indexed in MEDLINE, CINAHL, EMBASE, the Cochrane Library and Clarivate Analytics Science Citation Index.

This journal is a member of and subscribes to the principles of the Committee on Publication Ethics (COPE) (www.publicationethics.org/).

Editorial contact: journals.library@nihr.ac.uk

The full HTA archive is freely available to view online at www.journalslibrary.nihr.ac.uk/hta. Print-on-demand copies can be purchased from the report pages of the NIHR Journals Library website: www.journalslibrary.nihr.ac.uk

\section{Criteria for inclusion in the Health Technology Assessment journal}

Reports are published in Health Technology Assessment (HTA) if (1) they have resulted from work for the HTA programme, and (2) they are of a sufficiently high scientific quality as assessed by the reviewers and editors.

Reviews in Health Technology Assessment are termed 'systematic' when the account of the search appraisal and synthesis methods (to minimise biases and random errors) would, in theory, permit the replication of the review by others.

\section{HTA programme}

Health Technology Assessment (HTA) research is undertaken where some evidence already exists to show that a technology can be effective and this needs to be compared to the current standard intervention to see which works best. Research can evaluate any intervention used in the treatment, prevention or diagnosis of disease, provided the study outcomes lead to findings that have the potential to be of direct benefit to NHS patients. Technologies in this context mean any method used to promote health; prevent and treat disease; and improve rehabilitation or long-term care. They are not confined to new drugs and include any intervention used in the treatment, prevention or diagnosis of disease.

The journal is indexed in NHS Evidence via its abstracts included in MEDLINE and its Technology Assessment Reports inform National Institute for Health and Care Excellence (NICE) guidance. HTA research is also an important source of evidence for National Screening Committee (NSC) policy decisions.

\section{This report}

The research reported in this issue of the journal was funded by the HTA programme as project number $15 / 26 / 06$. The contractual start date was in October 2016. The draft report began editorial review in November 2020 and was accepted for publication in April 2021. The authors have been wholly responsible for all data collection, analysis and interpretation, and for writing up their work. The HTA editors and publisher have tried to ensure the accuracy of the authors' report and would like to thank the reviewers for their constructive comments on the draft document. However, they do not accept liability for damages or losses arising from material published in this report.

This report presents independent research funded by the National Institute for Health Research (NIHR). The views and opinions expressed by authors in this publication are those of the authors and do not necessarily reflect those of the NHS, the NIHR, NETSCC, the HTA programme or the Department of Health and Social Care. If there are verbatim quotations included in this publication the views and opinions expressed by the interviewees are those of the interviewees and do not necessarily reflect those of the authors, those of the NHS, the NIHR, NETSCC, the HTA programme or the Department of Health and Social Care.

Copyright $\odot 2021$ Hopewell et al. This work was produced by Hopewell et al. under the terms of a commissioning contract issued by the Secretary of State for Health and Social Care. This is an Open Access publication distributed under the terms of the Creative Commons Attribution CC BY 4.0 licence, which permits unrestricted use, distribution, reproduction and adaption in any medium and for any purpose provided that it is properly attributed. See: https://creativecommons.org/licenses/by/4.0/. For attribution the title, original author(s), the publication source - NIHR Journals Library, and the DOI of the publication must be cited.

Published by the NIHR Journals Library (www.journalslibrary.nihr.ac.uk), produced by Prepress Projects Ltd, Perth, Scotland (www.prepress-projects.co.uk). 
Professor Ken Stein Professor of Public Health, University of Exeter Medical School, UK

\section{NIHR Journals Library Editors}

Professor John Powell Chair of HTA and EME Editorial Board and Editor-in-Chief of HTA and EME journals. Consultant Clinical Adviser, National Institute for Health and Care Excellence (NICE), UK, and Professor of Digital Health Care, Nuffield Department of Primary Care Health Sciences, University of Oxford, UK

Professor Andrée Le May Chair of NIHR Journals Library Editorial Group (HS\&DR, PGfAR, PHR journals) and Editor-in-Chief of HS\&DR, PGFAR, PHR journals

Professor Matthias Beck Professor of Management, Cork University Business School, Department of Management and Marketing, University College Cork, Ireland

Dr Tessa Crilly Director, Crystal Blue Consulting Ltd, UK

Dr Eugenia Cronin Senior Scientific Advisor, Wessex Institute, UK

Dr Peter Davidson Consultant Advisor, Wessex Institute, University of Southampton, UK

Ms Tara Lamont Senior Scientific Adviser (Evidence Use), Wessex Institute, University of Southampton, UK

Dr Catriona McDaid Senior Research Fellow, York Trials Unit, Department of Health Sciences, University of York, UK

Professor William McGuire Professor of Child Health, Hull York Medical School, University of York, UK

Professor Geoffrey Meads Emeritus Professor of Wellbeing Research, University of Winchester, UK

Professor James Raftery Professor of Health Technology Assessment, Wessex Institute, Faculty of Medicine, University of Southampton, UK

Dr Rob Riemsma Reviews Manager, Kleijnen Systematic Reviews Ltd, UK

Professor Helen Roberts Professor of Child Health Research, UCL Great Ormond Street Institute of Child Health, UK

Professor Jonathan Ross Professor of Sexual Health and HIV, University Hospital Birmingham, UK

Professor Helen Snooks Professor of Health Services Research, Institute of Life Science, College of Medicine, Swansea University, UK

Professor Ken Stein Professor of Public Health, University of Exeter Medical School, UK

Professor Jim Thornton Professor of Obstetrics and Gynaecology, Faculty of Medicine and Health Sciences, University of Nottingham, UK

Please visit the website for a list of editors: www.journalslibrary.nihr.ac.uk/about/editors

Editorial contact: journals.library@nihr.ac.uk 


\title{
Abstract
}

\section{Progressive exercise compared with best-practice advice, with or without corticosteroid injection, for rotator cuff disorders: the GRASP factorial RCT}

\author{
Sally Hopewell@, ${ }^{1 *}$ David J Keene®,$^{2}$ Peter Heine ${ }^{2},{ }^{2}$ Ioana R Marian®,$^{1}$ \\ Melina Dritsakiø, ${ }^{1}$ Lucy Cureton $\odot,{ }^{2}$ Susan J Dutton $\odot,{ }^{1}$ Helen Dakin $\odot,{ }^{3}$ \\ Andrew Carr®, ${ }^{2}$ Willie Hamilton $\odot,{ }^{4}$ Zara Hansen $\odot,{ }^{2}$ Anju Jaggi®, ${ }^{5}$ \\ Chris Littlewood@, ${ }^{6}$ Karen Barker@, ${ }^{2}$ Alastair Gray ${ }^{3}$ and Sarah E Lamb ${ }^{2,4}$ \\ on behalf of the GRASP Trial Group
}

\author{
1Oxford Clinical Trials Research Unit, Nuffield Department of Orthopaedics, Rheumatology and \\ Musculoskeletal Sciences, University of Oxford, Oxford, UK \\ ${ }^{2}$ Nuffield Department of Orthopaedics, Rheumatology and Musculoskeletal Sciences, University of \\ Oxford, Oxford, UK \\ ${ }^{3}$ Nuffield Department of Population Health, University of Oxford, Oxford, UK \\ ${ }^{4}$ College of Medicine and Health, University of Exeter, Exeter, UK \\ ${ }^{5}$ Royal National Orthopaedic Hospital NHS Trust, Stanmore, UK \\ 6 Manchester Metropolitan University, Manchester, UK \\ *Corresponding author sally.hopewell@csm.ox.ac.uk
}

Background: Rotator cuff-related shoulder pain is very common, but there is uncertainty regarding which modes of exercise delivery are optimal and the long-term benefits of corticosteroid injections.

Objectives: To assess the clinical effectiveness and cost-effectiveness of progressive exercise compared with best-practice physiotherapy advice, with or without corticosteroid injection, in adults with a rotator cuff disorder.

Design: This was a pragmatic multicentre superiority randomised controlled trial (with a $2 \times 2$ factorial design).

Setting: Twenty NHS primary care-based musculoskeletal and related physiotherapy services.

Participants: Adults aged $\geq 18$ years with a new episode of rotator cuff-related shoulder pain in the previous 6 months.

Interventions: A total of 708 participants were randomised (March 2017-May 2019) by a centralised computer-generated 1:1:1:1 allocation ratio to one of four interventions: (1) progressive exercise ( $n=174)$ (six or fewer physiotherapy sessions), (2) best-practice advice $(n=174)$ (one physiotherapy session), (3) corticosteroid injection then progressive exercise $(n=182)$ (six or fewer physiotherapy sessions) or (4) corticosteroid injection then best-practice advice $(n=178)$ (one physiotherapy session).

Main outcome measures: The primary outcome was Shoulder Pain and Disability Index (SPADI) score over 12 months. Secondary outcomes included SPADI subdomains, the EuroQol 5 Dimensions, five-level version, sleep disturbance, fear avoidance, pain self-efficacy, return to activity, Global Impression of Treatment and health resource use. Outcomes were collected by postal questionnaires at 8 weeks and at 6 and 12 months. A within-trial economic evaluation was also conducted. The primary analysis was intention to treat. 
Results: Participants had a mean age of 55.5 (standard deviation 13.1) years and 49.3\% were female. The mean baseline SPADI score was 54.1 (standard deviation 18.5). Follow-up rates were $91 \%$ at 8 weeks and $87 \%$ at 6 and 12 months. There was an overall improvement in SPADI score from baseline in each group over time. Over 12 months, there was no evidence of a difference in the SPADI scores between the progressive exercise intervention and the best-practice advice intervention in shoulder pain and function (adjusted mean difference between groups over 12 months $-0.66,99 \%$ confidence interval -4.52 to 3.20). There was also no difference in SPADI scores between the progressive exercise intervention and best-practice advice intervention when analysed at the 8-week and 6- and 12-month time points. Injection resulted in improvement in shoulder pain and function at 8 weeks compared with no injection (adjusted mean difference $-5.64,99 \%$ confidence interval -9.93 to -1.35 ), but not when analysed over 12 months (adjusted mean difference -1.11 , 99\% confidence interval -4.47 to 2.26 ), or at 6 and 12 months. There were no serious adverse events. In the base-case analysis, adding injection to best-practice advice gained 0.021 quality-adjusted life-years $(p=0.184)$ and increased the cost by $£ 10$ per participant $(p=0.747)$. Progressive exercise alone was $£ 52(p=0.247)$ more expensive per participant than best-practice advice, and gained 0.019 QALYs ( $p=0.220)$. At a ceiling ratio of $£ 20,000$ per quality-adjusted life-year, injection plus best-practice advice had a $54.93 \%$ probability of being the most cost-effective treatment.

Limitations: Participants and physiotherapists were not blinded to group allocation. Twelve-month follow-up may be insufficient for identifying all safety concerns.

Conclusions: Progressive exercise was not superior to a best-practice advice session with a physiotherapist. Subacromial corticosteroid injection improved shoulder pain and function, but provided only modest short-term benefit. Best-practice advice in combination with corticosteroid injection was expected to be most cost-effective, although there was substantial uncertainty.

Future work: Longer-term follow-up, including any serious adverse effects of corticosteroid injection.

Trial registration: Current Controlled Trials ISRCTN16539266 and EudraCT 2016-002991-28.

Funding: This project was funded by the National Institute for Health Research (NIHR) Health Technology Assessment programme and will be published in full in Health Technology Assessment; Vol. 25, No. 48. See the NIHR Journals Library website for further project information. 


\section{Contents}

List of tables $\quad$ xiii

List of figures $\quad$ xvii

List of abbreviations $\quad$ xix

Plain English summary $\quad$ xxi

Scientific summary $\quad$ xxiii

Chapter 1 Introduction 1

Scientific background 1

Problem and diagnosis 1

Explanation of rationale 1

Choice of comparators 2

Exercise interventions 2

Corticosteroid injection 3

Objectives 3

Chapter 2 Methods $\quad 5$

Trial design 5

Internal pilot $\quad 5$

Study setting $\quad 6$

Participants 6

Inclusion criteria 6

Exclusion criteria $\quad 6$

Recruitment 6

Recruitment of participants, screening and eligibility assessment 6

$\begin{array}{ll}\text { Informed consent and baseline assessment } & 7\end{array}$

Randomisation 8

Blinding 8

Interventions 8

$\begin{array}{lr}\text { Subacromial corticosteroid injection } & 8\end{array}$

Progressive exercise intervention 9

Best-practice advice intervention 9

Concomitant care 9

Training and monitoring of intervention delivery 9

$\begin{array}{lr}\text { Outcomes } & 10\end{array}$

$\begin{array}{ll}\text { Primary outcome } & 10\end{array}$

Secondary outcomes 10

$\begin{array}{ll}\text { Adverse events } & 11\end{array}$

Follow-up data collection $\quad 11$

Data management 12

Statistical methods 12

Sample size 12 
$\begin{array}{lr}\text { Statistical analysis } & 12\end{array}$

$\begin{array}{ll}\text { Interaction } & 13\end{array}$

$\begin{array}{ll}\text { Primary outcome analysis } & 14\end{array}$

$\begin{array}{ll}\text { Secondary outcome analyses } & 14\end{array}$

$\begin{array}{ll}\text { Missing data } & 14\end{array}$

$\begin{array}{ll}\text { Sensitivity analysis } & 14\end{array}$

Prespecified subgroup analysis $\quad 14$

$\begin{array}{ll}\text { Supplementary/additional analyses } & 15\end{array}$

$\begin{array}{lr}\text { Cost-effectiveness analysis } & 15\end{array}$

Patient and public involvement 15

Ethics approval and monitoring 16

$\begin{array}{ll}\text { Ethics committee approval } & 16\end{array}$

Trial Management Group 16

Trial Steering Committee 16

Data Monitoring and Ethics Committee 16

$\begin{array}{ll}\text { Chapter } 3 \text { Intervention description and rationale } & 17\end{array}$

Developing the GRASP trial exercise interventions 17

Intervention development 17

$\begin{array}{ll}\text { Internal pilot } & 18\end{array}$

The GRASP trial interventions 18

GRASP trial exercise interventions $\quad 21$

Strategies to encourage exercise adherence 26

Monitoring, quality assurance and safety 26

$\begin{array}{ll}\text { Conclusion } & 27\end{array}$

Chapter 4 Results 29

Trial sites 29

$\begin{array}{ll}\text { Centre characteristics } & 29\end{array}$

$\begin{array}{ll}\text { Participating clinicians } & 29\end{array}$

Participant flow 29

Recruitment 29

$\begin{array}{lr}\text { Screening } & 29\end{array}$

Baseline data 32

Baseline characteristics of participants $\quad 32$

Follow-up 35

Withdrawals $\quad 35$

Intervention delivery $\quad 35$

Compliance with intervention $\quad 35$

$\begin{array}{ll}\text { Timing of delivery of interventions } & 37\end{array}$

$\begin{array}{ll}\text { Content and adherence to interventions } & 37\end{array}$

Best-practice advice intervention 38

Progressive-exercise intervention 38

Exercise progression $\quad 39$

Participant-reported adherence to exercise 41

Quality assurance $\quad 41$

Outcomes and estimations $\quad 41$

Primary outcome: shoulder pain and function - SPADI 41

Interaction 41

SPADI: progressive exercise compared with best-practice advice $\quad 42$

SPADI: injection compared with no injection 42 
$\begin{array}{ll}\text { Sensitivity analysis } & 44\end{array}$

Complier-average causal effect analysis $\quad 44$

Progressive exercise compared with best-practice advice 44

Injection compared with no injection 44

Secondary outcomes 44

Progressive exercise compared with best-practice advice 45

SPADI: shoulder pain subscale $\quad 46$

SPADI: shoulder function subscale $\quad 46$

Fear Avoidance Belief Questionnaire - Physical Activity 46

Pain Self-efficacy Questionnaire $\quad 47$

Insomnia Severity Index $\quad 47$

Return to desired activities $\quad 47$

Global Impression of Treatment $\quad 47$

$\begin{array}{ll}\text { Injection compared with no injection } & 47\end{array}$

$\begin{array}{ll}\text { SPADI: shoulder pain subscale } & 47\end{array}$

SPADI: shoulder function subscale $\quad 49$

Fear Avoidance Belief Questionnaire - Physical Activity 49

Pain Self-efficacy Questionnaire 49

Insomnia Severity Index $\quad 50$

Return to desired activities $\quad 50$

Global Impression of Treatment 50

Other outcomes 50

Participant-reported shoulder condition $\quad 50$

Participant-reported injection outside the trial $\quad 50$

Harms 50

Missing data 51

Prespecified subgroup analysis $\quad 51$

Progressive exercise compared with best-practice advice 51

Injection compared with no injection $\quad 52$

Chapter 5 Health economics 53

Introduction $\quad 53$

Methods 53

Aim 53

Measurement of resource use and costs 53

Costing of the interventions $\quad 54$

Subacromial corticosteroid injection $\quad 54$

Best-practice advice $\quad 54$

Progressive exercise $\quad 54$

Training $\quad 55$

Base-case analysis $\quad 56$

Worst-case scenario $\quad 57$

Best-case scenario $\quad 57$

Measurement of broader resource use $\quad 57$

Valuation of resource use $\quad 58$

Calculation of utilities and quality-adjusted life-years 58

Missing data $\quad 58$

Analyses of resource use, costs and outcome data 59

Cost-effectiveness analysis $\quad 59$

Regression analysis with interaction term (base-case analysis) 59

Regression analysis without interaction term (sensitivity analysis) 60 
$\begin{array}{ll}\text { Results of economic analysis } & 60\end{array}$

$\begin{array}{ll}\text { Completion rate } & 60\end{array}$

$\begin{array}{ll}\text { Cost-effectiveness results } & 64\end{array}$

Base-case results $\quad 64$

$\begin{array}{ll}\text { Discussion } & 66\end{array}$

$\begin{array}{ll}\text { Chapter } 6 \text { Discussion } & 69\end{array}$

Interpretation 69

Aim and overview of trial findings $\quad 69$

$\begin{array}{ll}\text { Generalisability } & 70\end{array}$

$\begin{array}{ll}\text { Internal validity and methodology } & 70\end{array}$

$\begin{array}{ll}\text { External validity and generalisability of study findings } & 71\end{array}$

Overall evidence: comparison with other literature $\quad 72$

$\begin{array}{ll}\text { Exercise intervention } & 72\end{array}$

$\begin{array}{ll}\text { Corticosteroid injection } & 73\end{array}$

$\begin{array}{ll}\text { Chapter } 7 \text { Conclusion } & 75\end{array}$

$\begin{array}{ll}\text { Implications for health care } & 75\end{array}$

$\begin{array}{ll}\text { Implications for research } & 76\end{array}$

$\begin{array}{ll}\text { Acknowledgements } & 77\end{array}$

$\begin{array}{ll}\text { References } & 81\end{array}$

$\begin{array}{ll}\text { Appendix } 1 \text { Chapter } 1 \text { appendix } & 91\end{array}$

$\begin{array}{ll}\text { Appendix } 2 \text { Chapter } 2 \text { appendix } & 93\end{array}$

$\begin{array}{ll}\text { Appendix } 3 \text { Chapter } 3 \text { appendix } & 95\end{array}$

$\begin{array}{ll}\text { Appendix } 4 \text { Chapter } 4 \text { appendix } & 105\end{array}$

$\begin{array}{ll}\text { Appendix } 5 \text { Chapter } 5 \text { appendix } & 125\end{array}$

$\begin{array}{ll}\text { Appendix } 6 \text { Chapter } 6 \text { appendix } & 141\end{array}$ 


\section{List of tables}

$\begin{array}{ll}\text { TABLE } 1 \text { Outcomes measured and time points assessed } & 7\end{array}$

$\begin{array}{ll}\text { TABLE } 2 \text { Factorial } 2 \times 2 \text { analysis diagram } & 13\end{array}$

TABLE 3 Overview of GRASP trial exercise interventions, as per Template for Intervention Description and Replication criteria 18

TABLE 4 Contents of the GRASP trial participant information booklet 21

$\begin{array}{ll}\text { TABLE } 5 \text { Number of participants screened by site } & 31\end{array}$

TABLE 6 Participant baseline characteristics ('inside the table') 32

TABLE 7 Participant withdrawals and reason for withdrawal 35

$\begin{array}{ll}\text { TABLE } 8 \text { Intervention received } & 36\end{array}$

TABLE 9 Content of subacromial corticosteroid injection 38

TABLE 10 Content of best-practice advice intervention 39

TABLE 11 Content of progressive-exercise intervention 40

TABLE 12 Shoulder Pain and Disability Index summary at each time point by randomised group 42

TABLE 13 Progressive exercise vs. best-practice advice at-the-margins analysis of SPADI 43

TABLE 14 Injection vs. no injection at-the-margins analysis of SPADI 43

TABLE 15 Analysis of secondary outcomes for progressive exercise vs. best-practice advice at 8 weeks, 6 months and 12 months

TABLE 16 Analysis of secondary outcomes for injection vs. no injection at 8 weeks, 6 months and 12 months

TABLE 17 Unit cost of consumables associated with trial per participant

TABLE 20 Completion rate (\%) of health resource use by treatment interventions and follow-up time points

TABLE 21 Response rate (\%) of EQ-5D-5L by follow-up time point and treatment

TABLE 22 Health-care cost $(£)$ over the 12-month follow-up (available cases, without imputation of missing data) 
TABLE 23 Non-NHS costs (£) over the 12 months (available cases, without imputation of missing data)

TABLE 24 Utility and QALY estimates: EQ-5D-5L scores (available cases with and without imputation and adjustment for baseline utility)

TABLE 25 Regression analysis with an interaction term, including imputation of missing values and adjustment for baseline utility, sex and age (base-case analysis)

TABLE 26 Categories of exercise considered by the delegates of the GRASP trial intervention development meeting

TABLE 27 Behavioural strategies to address barriers to exercise, highlighting common and discrete components to each exercise intervention

TABLE 28 Characteristics of participating NHS trusts

TABLE 29 Characteristics of participating physiotherapists

TABLE 30 Reasons participants were ineligible or declined to participate

TABLE 31 Baseline characteristics detailed (inside the table)

TABLE 32 Participant demographics at baseline: categorical outcomes (at the margins)

TABLE 33 Participant demographic characteristics at baseline: continuous outcomes (at the margins)

TABLE 34 Stratification factors (at the margins)

TABLE 35 Response rates

TABLE 36 Timing of intervention delivery

TABLE 37 Exercises prescribed: progressive-exercise intervention

TABLE 38 Progression of exercise intervention: progressive-exercise intervention

TABLE 39 Participant-reported exercise adherence

TABLE 40 Progressive exercise vs. best-practice advice and injection vs. best-practice advice: inside-the-table analysis of SPADI overall

TABLE 41 Progressive exercise vs. best-practice advice and injection vs. best-practice advice: inside-the-table analysis of SPADI at each time point

TABLE 42 Secondary outcomes analysis: unadjusted mean and SD

TABLE 43 Summary of prescribed medication unit cost (in $2019 £$ )

TABLE 44 Unit costs of health and social care items and additional financial cost due to rotator cuff disorder 
TABLE 45 Mean health resource utilisation: baseline to 8 weeks (available-case analysis, excluding patients with missing data on that resource)

TABLE 46 Mean health resource utilisation: 8 weeks to 6 months (available-case analysis, excluding patients with missing data on that resource)

TABLE 47 Mean health resource utilisation: 6-12 months (available-case analysis, excluding patients with missing data on that resource)

TABLE 48 Mean NHS costs (SD) by treatment group and follow-up time point (2019 prices): baseline to 8 weeks (available cases)

TABLE 49 Mean NHS costs (SD) by treatment group and follow-up time point (2019 prices): 8 weeks to 6 months (available cases)

TABLE 50 Mean NHS costs (SD) by treatment group and follow-up time point (2019 prices): 6-12 months (available cases)

TABLE 51 Mean prescribed drug cost

TABLE 52 Utilisation of steroid injections outside the GRASP trial

TABLE 53 Utility and QALY estimates: EQ-5D visual analogue scale scores (available cases with no adjustment for baseline utility)

TABLE 54 Regression analysis without interaction term, including imputation of missing values and adjustment for baseline utility, sex and age

TABLE 55 Regression analysis with an interaction term, including both NHS and broader societal perspective

TABLE 56 Results of the worst-case scenario for training costs: regression analysis with an interaction term, including imputation of missing values and adjustment for baseline utility, sex and age

TABLE 57 Results of the best-case scenario: regression analysis with an interaction term, including imputation of missing values and adjustment for baseline utility, sex and age

TABLE 58 Summary of trials comparing the effects of supervised vs. unsupervised exercise interventions

TABLE 59 Studies comparing subacromial corticosteroid injection with no injection or placebo injection 



\section{List of figures}

FIGURE 1 Process map of the GRASP trial progressive exercise intervention

FIGURE 2 Guidance for setting level 2 resistance exercises in the GRASP trial

FIGURE 3 Participant flow diagram

FIGURE 4 Marginal adjusted mean SPADI values from the repeated measures mixed-effects model and associated $99 \% \mathrm{Cls}$ for the two treatment groups from baseline to 12 months: best-practice advice vs. progressive exercise

FIGURE 5 Marginal adjusted mean SPADI values from the repeated measures mixed-effects model and associated $99 \% \mathrm{Cls}$ for the two treatment groups from baseline to 12 months: injection vs. no injection

FIGURE 6 Marginal adjusted mean SPADI pain values from the repeated measures mixed-effects model and associated $95 \%$ Cls for the two treatment groups from baseline to 12 months: best-practice advice vs. progressive exercise

FIGURE 7 Marginal adjusted mean SPADI function values from the repeated measures mixed-effects model and associated $95 \% \mathrm{Cls}$ for the two treatment groups from baseline to 12 months: best-practice advice vs. progressive exercise

FIGURE 8 Marginal adjusted mean SPADI pain values from the repeated measures mixed-effects model and associated $95 \% \mathrm{Cls}$ for the two treatment groups from baseline to 12 months: injection vs. no injection

FIGURE 9 Marginal adjusted mean SPADI function values from the repeated measures mixed-effects model and associated $95 \% \mathrm{Cls}$ for the two treatment groups from baseline to 12 months: injection vs. no injection

FIGURE 10 Subgroup-adjusted SPADI analysis for progressive exercise over 12 months

FIGURE 11 Subgroup-adjusted SPADI analysis for injection over 12 months

FIGURE 12 The CEAC for the comparison between treatment groups (base-case analysis)

FIGURE 13 The BESS diagnostic algorithm

FIGURE 14 Injection referral form and treatment log

FIGURE 15 Progressive exercise intervention: action planner

FIGURE 16 Progressive exercise intervention: exercise diary

FIGURE 17 Progressive exercise intervention: level 1 simple shoulder movement exercises 
FIGURE 19 Progressive exercise intervention: level 2 optional resistance exercises

FIGURE 20 Progressive exercise intervention: level 2 optional resistance exercises

FIGURE 21 Best-practice advice intervention: exercise progression ladder

FIGURE 22 Best-practice advice intervention: exercise diary

FIGURE 23 Best-practice advice intervention: action planner

FIGURE 24 Predicted vs. actual recruitment rate per month

FIGURE 25 Subgroup-adjusted SPADI analysis for progressive exercise vs. best-practice advice at 8 weeks

FIGURE 26 Subgroup-adjusted SPADI analysis for progressive exercise vs. best-practice advice at 6 months

FIGURE 27 Subgroup-adjusted SPADI analysis for progressive exercise vs.

best-practice advice at 12 months

FIGURE 28 Subgroup-adjusted SPADI analysis for injection vs. no injection at 8 weeks

FIGURE 29 Subgroup-adjusted SPADI analysis for injection vs. no injection at 6 months

FIGURE 30 Subgroup-adjusted SPADI analysis for injection vs. no injection at 12 months 


\section{List of abbreviations}

\begin{tabular}{|c|c|c|c|}
\hline BESS & British Elbow \& Shoulder Society & ISI & Insomnia Severity Index \\
\hline \multirow{4}{*}{$\begin{array}{l}\text { BNF } \\
\text { CACE } \\
\text { CEAC }\end{array}$} & British National Formulary & ITT & intention to treat \\
\hline & \multirow{3}{*}{$\begin{array}{l}\text { complier-average causal effect } \\
\text { cost-effectiveness acceptability } \\
\text { curve }\end{array}$} & MCID & minimally clinically important \\
\hline & & & difterence \\
\hline & & MD & mean difference \\
\hline \multirow{3}{*}{$\begin{array}{l}\mathrm{Cl} \\
\mathrm{CINHAL}\end{array}$} & \multirow{3}{*}{$\begin{array}{l}\text { confidence interval } \\
\text { Cumulative Index to Nursing and } \\
\text { Allied Health Literature }\end{array}$} & NICE & National Institute for Health and \\
\hline & & & Care Excellence \\
\hline & & NIHR & National Institute for Health \\
\hline DMEC & $\begin{array}{l}\text { Data Monitoring and Ethics } \\
\text { Committee }\end{array}$ & NMB & net monetary benefit \\
\hline & digital versatile disc & OCTRU & Oxford Clinical Trials Research \\
\hline EQ-5D & EuroQol-5 Dimensions & & \\
\hline \multirow{2}{*}{ EQ-5D-5L } & \multirow{2}{*}{$\begin{array}{l}\text { EuroQol-5 Dimensions, five-level } \\
\text { version }\end{array}$} & PPI & patient and public involvement \\
\hline & & PSEQ-2 & $\begin{array}{l}\text { Pain Self-Efficacy Questionnaire, } \\
\text { two-item version }\end{array}$ \\
\hline FABQ & $\begin{array}{l}\text { Fear Avoidance Belief } \\
\text { Questionnaire }\end{array}$ & PSS & Personal Social Services \\
\hline \multirow[t]{2}{*}{ FABQ-PA } & \multirow{2}{*}{$\begin{array}{l}\text { Fear Avoidance Belief } \\
\text { Questionnaire - Physical Activity }\end{array}$} & QALY & quality-adjusted life-year \\
\hline & & RDA & return to desired activities \\
\hline GCP & Good Clinical Practice & RPE & Rating of Perceived Exertion \\
\hline GIT & \multirow{2}{*}{$\begin{array}{l}\text { Global Impression of Treatment } \\
\text { general practitioner }\end{array}$} & SAE & serious adverse event \\
\hline GP & & SAP & statistical analysis plan \\
\hline GRASP & $\begin{array}{l}\text { Getting it Right: Addressing } \\
\text { Shoulder Pain }\end{array}$ & SD & standard deviation \\
\hline \multirow{3}{*}{$\begin{array}{l}\text { HTA } \\
\text { ICER }\end{array}$} & \multirow{3}{*}{$\begin{array}{l}\text { Health Technology Assessment } \\
\text { incremental cost-effectiveness } \\
\text { ratio }\end{array}$} & SE & standard error \\
\hline & & SMD & standardised mean difference \\
\hline & & SPADI & Shoulder Pain and Disability Index \\
\hline IQR & interquartile range & TSC & Trial Steering Committee \\
\hline
\end{tabular}





\section{Plain English summary}

he rotator cuff is a group of muscles and tendons that stabilise the shoulder and allow it to move.
Problems with the rotator cuff are very common. Symptoms include pain, which can affect a
person's ability to work, sleep well or perform daily tasks. It is not known which treatments work best
for shoulder pain, how exactly they should be delivered and whether or not people do better if they
are given a steroid injection.

The GRASP (Getting it Right: Addressing Shoulder Pain) trial tested whether or not people with a rotator cuff disorder would do better after a progressive exercise programme (supervised by a physiotherapist over six appointments spread out over 16 weeks) compared with a one-off best-practice advice session with a physiotherapist. The trial also tested whether or not giving a corticosteroid injection in the shoulder before starting either regime would help people recover more. We assessed the cost of delivering these treatments to the NHS.

We recruited 708 people from 20 NHS-based musculoskeletal centres in the UK. People were allocated to one of four treatment groups at random: (1) progressive exercise (six or fewer physiotherapy sessions), (2) best-practice advice (one physiotherapy session), (3) corticosteroid injection then progressive exercise (six or fewer physiotherapy sessions) or (4) corticosteroid injection then best-practice advice (one physiotherapy session). Trial participants were asked to complete a questionnaire that asked about their level of shoulder pain and their ability to perform basic daily tasks before treatment, and then again at 8 weeks and at 6 and 12 months.

Participants' shoulder pain and function improved over time in each of the four treatment groups. The GRASP trial showed that there was no difference between the best-practice advice session with a physiotherapist and the more comprehensive exercise programme. Corticosteroid injection improved people's shoulder pain and function, but only by a small amount and in the short term. No serious side effects were observed during the 12-month follow-up period. Best-practice advice in combination with corticosteroid injection is likely to be most cost-effective to the NHS. 



\section{Scientific summary}

\section{Background}

Shoulder pain is very common, with around $70 \%$ of cases due to rotator cuff-related shoulder pain. Despite the widespread use of physiotherapy, there is uncertainty regarding which type of exercise therapy is associated with the best outcomes. There is also uncertainty about the long-term benefits and harms of corticosteroid injection therapy, which is often used in addition to physiotherapy.

\section{Objectives}

The GRASP (Getting it Right: Addressing Shoulder Pain) trial assessed (1) if an individually tailored progressive home exercise programme prescribed and supervised by a physiotherapist provided greater improvement in shoulder pain and function over 12 months compared with a best-practice advice session with a physiotherapist supported by high-quality self-management materials; and (2) if subacromial corticosteroid injection provided greater improvement in shoulder pain and function over 12 months compared with no injection.

\section{Methods}

\section{Design}

This was a pragmatic multicentre superiority randomised controlled trial using a $2 \times 2$ factorial design. Participants and physiotherapists were not blinded to group allocation.

\section{Setting}

Participants were recruited from 20 NHS primary care-based musculoskeletal and related physiotherapy services.

\section{Participants}

Adults aged $\geq 18$ years with a new episode of shoulder pain (i.e. in the previous 6 months) attributable to a rotator cuff disorder (e.g. cuff tendonitis, impingement syndrome, tendinopathy or rotator cuff tear), as per British Elbow \& Shoulder Society guidelines, not currently receiving physiotherapy or being considered for surgery.

\section{Interventions}

Participants $(n=708)$ were randomised (March 2017-May 2019) using a centralised computergenerated $1: 1: 1: 1$ allocation ratio to one of four interventions: (1) progressive exercise $(n=174)$ (six or fewer physiotherapy sessions), (2) best-practice advice $(n=174)$ (one physiotherapy session), (3) corticosteroid injection then progressive exercise $(n=182)$ (six or fewer physiotherapy sessions) or (4) corticosteroid injection then best-practice advice $(n=178)$ (one physiotherapy session).

Participants randomised to the progressive exercise intervention received up to six individual face-to-face sessions with a physiotherapist over 16 weeks. Participants were provided with a folder containing an advice booklet, an exercise action planner and diary, and instructions on their exercise programme, which was set up in collaboration with their physiotherapist. A resistance band was issued as required. The progressive exercise programme was highly structured, but could be tailored to the needs and preferences of participants. 
Participants randomised to the best-practice advice intervention received a single individual face-to-face session with a physiotherapist. Participants were given an advice booklet. The content of the advice in the booklet was the same as that provided for the progressive exercise group, with the exception of a different exercise programme. Participants were given a simple set of self-guided exercises, at least one level of resistance band and access to an exercise video (available on a website and a digital versatile disc), which could be progressed and regressed, depending on their capability. The exercises were designed using similar concepts to the progressive exercise intervention, such as increased resistance, but these were a simpler range. An exercise diary was provided in addition to an exercise action planner that was simpler than the one provided to those in the progressive exercise group.

\section{Follow-up}

Measurements for the primary and secondary outcomes were collected by postal questionnaires at 8 weeks and at 6 and 12 months after randomisation. Telephone follow-up was used to contact those who did not respond or fully complete the returned questionnaire.

\section{Clinical outcomes and analysis}

The primary outcome was the mean difference in Shoulder Pain and Disability Index (SPADI) total score over 12 months. The scale is from 0 to 100, with higher values representing worse pain. Secondary outcomes were the pain and function SPADI subdomains, health-related quality of life (assessed using the EuroQol-5 Dimensions, five-level version), sleep disturbance, fear avoidance, pain self-efficacy, return to activity, global impression of treatment, health resource use, out-of-pocket expenses and work disability. Prespecified subgroup analyses included age, sex, smoking status, higher baseline SPADI score $(\geq 50)$ and higher baseline pain self-efficacy score $(\geq 8)$. The planned sample size was 704 participants, assuming 20\% loss to follow-up at 12 months, and based on $90 \%$ power and $1 \%$ two-sided statistical significance to detect a minimally clinically important difference of eight points on the SPADI total scale. The primary analysis was intention to treat. The two main effect comparisons for this $2 \times 2$ factorial trial were (1) progressive exercise compared with best-practice advice to determine the efficacy of progressive exercise and (2) subacromial corticosteroid injection compared with no injection to determine the efficacy of subacromial corticosteroid injection. The presence of an interaction effect was formally investigated before testing their effects on the primary outcome. The difference in SPADI score between the two intervention groups was estimated overall and at each data collection time point using a repeated measures linear mixed-effects regression model adjusted for baseline and other covariates.

\section{Economic analysis}

The cost-utility of interventions was evaluated from an NHS and Personal Social Services perspective, using a within-trial intention-to-treat analysis. Quality-adjusted life-years were estimated from data collected from the EuroQol-5 Dimensions, five-level version, at baseline, 8 weeks and 6 and 12 months. Costs were estimated for each participant over 12 months of follow-up based on patientreported use of health-care services attributable to their rotator cuff disorder. The cost of delivering each intervention, including physiotherapists' training, materials, delivery of the progressive exercise and advice sessions, and corticosteroid injections, was also estimated.

\section{Results}

The mean age of participants was 55.5 (standard deviation 13.1) years, $49.3 \%$ of participants were female and the mean duration of symptoms was 4 (interquartile range 3-6) months. Intervention groups were well matched in terms of demographic data and clinical and generic health-related quality-of-life measures. Overall, 92\% (324/352) of participants randomised to the best-practice advice intervention and $95 \%$ (339/356) of participants allocated to progressive exercise either partially or fully completed the intervention. High levels of protocol adherence were achieved across all intervention groups. Follow-up data were obtained for $87 \%$ (618/708), 87\% (615/708) and $91 \%(641 / 708)$ of participants at 12 months, 6 months and 8 weeks, respectively. 
The overall mean baseline SPADI score was 54.1 (standard deviation 18.5), with higher baseline levels of shoulder pain (mean SPADI pain subscale score 63.9; standard deviation 17.1) than impaired function (mean SPADI function subscale score 44.3; standard deviation 22.1). There was an overall improvement in SPADI score in each of the four groups from baseline over time, representing a 32.2-point improvement (standard deviation 23.9 points) on the SPADI scale [with a SPADI score of 21.9 (standard deviation 23.4) at 12 months]. There was no evidence of an interaction effect and so results were analysed for the two main effect comparisons.

\section{Clinical results}

Over 12 months, there was no evidence of a difference in the SPADI scores between the progressive exercise intervention and best-practice advice intervention (adjusted mean difference between groups over 12 months -0.66 , $99 \%$ confidence interval -4.52 to 3.20 ); nor was there evidence of a difference when analysed at the 8-week and 6- and 12-month time points (adjusted mean difference at 12 months $-3.10,99 \%$ confidence interval -7.85 to 1.64 ). There was also no difference between groups for secondary outcome measures, with the exception of progressive exercise, which resulted in an improvement in patient-reported global impression of treatment over the 12 months (adjusted mean difference over 12 months $0.38,95 \%$ confidence interval 0.10 to 0.66 ) and at the 6 - and 12 -month time points.

Over 12 months, there was also no evidence of a difference in SPADI scores between the injection and the no injection groups (adjusted mean difference over 12 months -1.11 , 99\% confidence interval -4.47 to 2.26). There was a small difference in SPADI scores at 8 weeks (adjusted mean difference at 8 weeks $-5.64,99 \%$ confidence interval -9.93 to -1.35 ) in favour of the injection group, but not at the 6- and 12-month time points (adjusted mean difference at 12 months 1.93, 99\% confidence interval -2.41 to 6.27$)$. There was no difference between groups for secondary outcome measures, with the exception of the injection group at 8 weeks, which resulted in a small improvement in shoulder pain, shoulder function, sleep disturbance, return to desired activities and global impression of treatment.

Prespecified subgroup analysis showed that the effect of injection was stronger at 8 weeks in people with a higher baseline SPADI score (adjusted mean difference at 8 weeks $-9.67,99 \%$ confidence interval -15.37 to -3.97 ) than in those who received injections but had a lower baseline SPADI score (adjusted mean difference at 8 weeks $-0.36,99 \%$ confidence interval -8.87 to 6.16 ). No differences were observed for other prespecified subgroup analyses. No serious adverse events were associated with treatment interventions.

\section{Economics results}

The base-case cost-effectiveness analysis showed that, over the 12-month period, participants in the best-practice advice treatment group gained, on average, 0.74 quality-adjusted life-years ( $95 \%$ confidence interval 0.710 to 0.763 ) and an NHS cost of $£ 195$. Adding progressive exercise to best-practice advice resulted in a gain of an additional 0.019 quality-adjusted life-years $(p=0.220)$, compared with best-practice advice alone, at an additional cost of $£ 52(p=0.247)$. Adding corticosteroid injection to best-practice advice resulted in a gain of 0.021 quality-adjusted life-years $(p=0.184)$, compared with best-practice advice alone, and increased the cost by $£ 10$ per participant $(p=0.747)$. At a $£ 20,000$ per quality-adjusted life-year ceiling ratio, best-practice advice plus injection was found to have a $54.93 \%$ probability of being best value for money of the four treatments evaluated in the trial. Best-practice advice plus injection cost $£ 475.59$ per quality-adjusted life-year gained compared with best-practice advice alone, and strongly dominated progressive exercise alone and progressive exercise plus injection, being less costly and accruing more quality-adjusted life-years. Sensitivity analyses assuming additive effects, taking a societal perspective and varying the cost of training physiotherapists, confirmed the base-case conclusion that best-practice advice plus injection is expected to be best value for money at a ceiling ratio of $£ 20,000$ per quality-adjusted life-year, although there was substantial uncertainty around this conclusion in all analyses. 


\section{Conclusion}

\section{Implications for health care}

The GRASP trial shows that the progressive exercise intervention was not superior to a best-practice advice session with a physiotherapist. Subacromial corticosteroid injection improved shoulder pain and function at 8 weeks, but provided modest short-term benefit only, with the greatest benefit being in those with higher levels of pain and functional impairment. Best-practice advice in combination with corticosteroid injection has a $54.93 \%$ probability of being the most cost-effective intervention for the NHS.

\section{Recommendations for research}

There is a case to extend follow-up to assess long-term outcomes, as some participants still reported ongoing pain and impaired shoulder function at 12 months. There is a need to better understand the natural history of rotator cuff disorders, including whether symptoms resolve over an extended period or persist in the longer term. Longer-term follow-up would also address concerns regarding later surgery and corticosteroid injection, and potential long-term harm due to its possible effects on tendon structure.

\section{Trial registration}

This trial is registered as ISRCTN16539266 and EudraCT 2016-002991-28.

\section{Funding}

This project was funded by the National Institute for Health Research (NIHR) Health Technology Assessment programme and will be published in full in Health Technology Assessment; Vol. 25, No. 48. See the NIHR Journals Library website for further project information. 


\section{Chapter 1 Introduction}

\section{Scientific background}

\section{Problem and diagnosis}

Shoulder pain is common. Annually, around $1 \%$ of adults aged $>45$ years in primary care present with a new episode of shoulder pain, accounting for $2.4 \%$ of all general practitioner (GP) consultations in the UK. ${ }^{1}$ This is most commonly attributed to the rotator cuff, which causes around $70 \%$ of cases. ${ }^{2}$ The rotator cuff is a group of four muscles and their tendons/attachments. The rotator cuff actively moves and stabilises the shoulder joint, enabling a wide range of efficient movements at the shoulder. Disorders of the rotator cuff can be associated with substantial, persistent disability (e.g. being unable to dress independently) and pain. Rotator cuff disorders can persist for long periods. Up to half of those who present for treatment, particularly older people, continue to have pain and/or functional disturbance for up to 2 years. ${ }^{3}$

The majority of shoulder pain is managed in primary care or in musculoskeletal interface services by physiotherapists and GPs. Musculoskeletal interface services are led by specialist practitioners who manage patients with musculoskeletal disorders through assessment, treatment, investigation and by referring to appropriate health-care professionals. Musculoskeletal interface services aim to promote more community-based management options for patients, rather than traditional hospital-based secondary care, and provide a more efficient, cost-effective and sustainable model for dealing with high-volume conditions. Treatments for rotator cuff disorders aim to improve pain and function. Standard primary care options include rest, advice, analgesia, non-steroidal anti-inflammatory drugs, physiotherapy and corticosteroid injections. 4,5 However, usual care can be highly variable and there are no National Institute for Health and Care Excellence (NICE) clinical guidelines.

A diagnostic algorithm ${ }^{2}$ has been developed as part of the NICE-accredited standards developed by the British Elbow \& Shoulder Society (BESS) and other professional bodies (e.g. the Royal College of Surgeons, the Chartered Society of Physiotherapy and the British Orthopaedic Association) to confirm when a diagnosis of rotator cuff disorder is highly likely, based on a patient's history and simple shoulder tests $^{4}$ (see Appendix 1, Figure 13). The recommended tests have been selected with primary care application in mind, ${ }^{6}$ although they do require a reasonable degree of clinical skill. Imaging is not recommended in primary care because of the poor fit between structural change and symptomatic presentation. ${ }^{7}$ We have used the BESS algorithm (see Appendix 1, Figure 13) to define the entry criteria for the GRASP (Getting it Right: Addressing Shoulder Pain) trial, thereby ensuring that the trial is consistent with national guidance.

\section{Explanation of rationale}

Problems associated with rotator cuff disorders can seriously affect patient health and well-being. The prevalence of shoulder complaints in the UK is estimated at around $14 \%,{ }^{8}$ increasing with age ${ }^{1}$ and highest in those aged $\geq 60$ years. Shoulder problems are a significant cause of morbidity and disability in the general population and have a significant socioeconomic burden, as they affect an individual's capacity to work and ability to perform daily tasks and social activities. They have a significant impact on primary care services. The average cost per patient with a musculoskeletal condition in the NHS is $£ 461.13$ per head per year, ${ }^{9}$ with wide geographical variability. The estimated cost to the UK economy is $£ 7.4 \mathrm{~B}$ per year. 
The NHS currently invests considerable amounts of money in unproven therapies and corticosteroid injections. One common treatment prescribed for rotator cuff disorders is corticosteroid injection, which typically costs $£ 47-332$, depending on the mode of delivery (the cheapest of which is by a physiotherapist without ultrasound guidance). In comparison, a set of six physiotherapy sessions costs approximately $£ 206$ and an assessment and advice session costs $£ 45$ (see Chapter 5). It is important for the NHS to develop cost-effective, pragmatic methods for dealing with high-volume conditions. Rotator cuff disorders may be self-limiting if they are managed effectively in primary care, as patients can regain function and pain can be reduced. However, the consequences of poor initial management are an increased likelihood of recurrent or persistent problems in older age and the need for surgical intervention. $^{4}$

We planned to conduct a large, well-powered randomised controlled trial, using a factorial design, to co-test two interventions commonly used in the management of rotator cuff disorders in primary care: (1) progressive exercise delivered by a physiotherapist and (2) corticosteroid injection. We used a bestpractice advice session with a physiotherapist and no injection as the respective comparators. The interventions tested used current patient pathways for people with a rotator cuff disorder. We wanted to assess which of these interventions, or combination of interventions, are most clinically effective and cost-effective for the NHS. The primary outcome for the trial is shoulder pain and function, which is assessed using the well-validated Shoulder Pain and Disability Index (SPADI).10,11 The SPADI is a tool that was developed to measure current shoulder pain and disability in an outpatient setting.

\section{Choice of comparators}

\section{Exercise interventions}

In designing the trial, we looked at existing evidence regarding the choice of comparator interventions. There is promising evidence from small, short-term trials that physiotherapist-prescribed exercise is effective. ${ }^{12-14}$ However, there is a lack of evidence regarding its long-term clinical effectiveness and cost-effectiveness, ${ }^{12-14}$ despite the widespread provision of physiotherapy for these conditions. There is also uncertainty about which types of exercise and delivery mechanisms (e.g. supervised or home based) are associated with the best outcomes. ${ }^{12,13,15-17}$ This evidence is limited by problems in study design and choice of comparators. ${ }^{13}$ There are also competing ideologies around which exercise programmes should be considered to ensure a worthwhile trial. Resistance training to improve muscular strength, whether supervised or home based, has been identified as a core component of exercise for rotator cuff disorders, although there is no evidence that any specific programme is superior. ${ }^{18,19}$ Manipulation of the exercise volume and intensity is achieved by varying the frequency, load, number of sets, repetitions and rest intervals. ${ }^{20} \mathrm{~A}$ trial of strength training found that duration, specificity of exercises, progression criteria and individualisation (i.e. adjusting the programme to suit each participant) were also important. ${ }^{21} \mathrm{We}$ did not consider other forms of physiotherapy-led interventions, such as electrotherapy, acupuncture, soft tissue mobilisation, manipulation or stratified care, because of the lack of evidence of their efficacy. ${ }^{22,23}$

Little attention has been paid to the need for behavioural frameworks to enhance adherence to advice and exercise programmes and to tackle pain beliefs and behaviour in this context. ${ }^{24}$ Non-adherence to physiotherapy treatment is estimated to be up to $70 \% .{ }^{25}$ In a large trial of exercise for lower back pain that did not include a behavioural component to increase exercise adherence, only half of the participants attended the minimum number of treatment sessions. ${ }^{26}$ Risk factors for low adherence include low levels of physical activity, low self-efficacy, depression, anxiety, poor social support and greater perceived barriers to exercise. ${ }^{24}$ Some of these risk factors are modifiable in the context of a physiotherapy intervention. We have previous expertise in this area ${ }^{27}$ and planned to include a behavioural component as part of the progressive exercise intervention. 


\section{Corticosteroid injection}

There is systematic review evidence that, in comparison with placebo, corticosteroid injections have a short-term benefit in the shoulder, as in other areas of the body. However, there are some concerns about the longer-term benefits and harms of corticosteroid injections. ${ }^{28-30}$ The combination of injection and physiotherapy has intuitive appeal, with some evidence of an additive, but not interactive, effect in the short term (3-4 months). ${ }^{30-33}$ The longer-term benefits of injections require further study. We planned to use a no-injection comparison, as finding an inert robust placebo is challenging and, given the existing evidence, ${ }^{28-30}$ we believed that it was unethical and undesirable to progress a placebo arm in a large Phase III trial. In our study based in NHS musculoskeletal services, extended-scope physiotherapists typically deliver the corticosteroid injections. This is increasingly common practice in the NHS, where therapists undertake additional post-registration training to deliver injections, working within a local Patient Group Direction and/or becoming qualified non-medical independent prescribers. ${ }^{34}$ Although the use of ultrasound to guide injections in primary care has become increasingly common, evidence from the SUPPORT (SUbacromial imPingement syndrome and Pain: a randomised controlled trial Of exeRcise and injecTion) trial ${ }^{35}$ and other trials ${ }^{3}$ have demonstrated that it is no more effective than standard injection practice. Ultrasound guidance also substantially increases the cost and reduces the practicality of injection therapy. Therefore, we planned to deliver injections without the use of ultrasound guidance.

\section{Objectives}

The aim of the GRASP trial was to assess the clinical effectiveness and cost-effectiveness of individually tailored progressive exercise compared with best-practice advice, with or without corticosteroid injection, in patients with a new episode of a rotator cuff disorder. The primary objectives were to assess the following:

- whether or not an individually tailored progressive exercise programme, including behavioural change strategies and led by a physiotherapist, provides greater improvement in shoulder pain and function over the 12 months post randomisation compared with a best-practice advice session with a physiotherapist supported by high-quality materials

- whether or not subacromial corticosteroid injection provides greater improvement in shoulder pain and function over the 12 months post randomisation compared with no injection.

The secondary objectives of the GRASP trial were to investigate if there were any differences at 8 weeks and at 6 and 12 months in shoulder pain, shoulder function, health-related quality of life, fear avoidance, pain self-efficacy, sleep disturbance, return to desired activities (RDA) (including work, social life and sport activities), patients' global impression of change, adherence to exercises, use of medication (prescribed and over the counter), time off work, health resource use (consultation with primary and secondary care) and additional out-of-pocket expenses.

A parallel within-trial health economic analysis was also conducted. 



\section{Chapter 2 Methods}

Darts of this chapter have been reproduced with permission from Hopewell et al. ${ }^{36}$ This is an Open

Access article distributed in accordance with the terms of the Creative Commons Attribution (CC BY 4.0) license, which permits others to distribute, remix, adapt and build upon this work, for commercial use, provided the original work is properly cited. See: http://creativecommons.org/licenses/ by/4.0/. The text below includes minor additions and formatting changes to the original text.

Parts of this chapter have been reproduced with permission from Marian et al..$^{37}$ This article is licensed under a Creative Commons Attribution 4.0 International License, which permits use, sharing, adaptation, distribution and reproduction in any medium or format, as long as you give appropriate credit to the original author(s) and the source, provide a link to the Creative Commons licence, and indicate if changes were made. The images or other third party material in this article are included in the article's Creative Commons licence, unless indicated otherwise in a credit line to the material. If material is not included in the article's Creative Commons licence and your intended use is not permitted by statutory regulation or exceeds the permitted use, you will need to obtain permission directly from the copyright holder. To view a copy of this licence, visit http://creativecommons.org/licenses/by/4.0/. The Creative Commons Public Domain Dedication waiver (http://creativecommons.org/publicdomain/zero/1.0/) applies to the data made available in this article, unless otherwise stated in a credit line to the data.

\section{Trial design}

The GRASP trial protocol ${ }^{36}$ and the statistical analysis plan (SAP) have been reported previously. ${ }^{37}$

The GRASP trial is a $2 \times 2$ factorial trial, which was used to test the following four physiotherapy-led interventions:

1. Progressive exercise programme (i.e. an individually tailored, progressive, home exercise programme prescribed and supervised by a physiotherapist, involving up to six face-to-face sessions over 16 weeks).

2. best-practice advice (i.e. one face-to-face session with a physiotherapist and a home exercise programme supported by high-quality self-management materials).

3. Progressive exercise programme (as described above) preceded by a subacromial corticosteroid injection.

4. best-practice advice session (as described above) preceded by a subacromial corticosteroid injection.

A parallel within-trial health economic analysis was also conducted.

The factorial design allowed two primary comparisons, based on the assumption that there was no interaction effect: (1) progressive exercise programme compared with best-practice advice session and (2) subacromial corticosteroid injection compared with no injection.

\section{Internal pilot}

An internal pilot was included as an integral part of the GRASP trial design, which mirrored the procedures and logistics undertaken in the main GRASP trial. The purpose of the internal pilot was to test and refine the recruitment process and explore treatment acceptability. The decision to progress to the main trial was made in collaboration with the Trial Steering Committee (TSC) and the National Institute for Health Research (NIHR) Health Technology Assessment (HTA) programme based on a predefined progression criterion: reaching the target recruitment rate (42 participants) within the specified time frame (4 months). Data from the internal pilot trial contributed to the final analysis, as there were no substantive changes in design or delivery of the trial interventions. 


\section{Study setting}

The GRASP trial was conducted across 20 primary care-based musculoskeletal services and their related physiotherapy services in the NHS. These services treat people with a range of musculoskeletal conditions and are run by specialist practitioners, including extended-scope physiotherapists, GPs with a specialist interest in musculoskeletal conditions, clinical nurse specialists and, in some instances, rheumatologists and orthopaedic consultants. Sites were chosen so that they reflected a range of settings (urban and rural) and were able to deliver the trial interventions. The local principal investigator was responsible for the conduct of the research at their site.

\section{Participants}

Participants were recruited if referred by their GP or physiotherapist for treatment of a new, but not necessarily first, episode of shoulder pain attributable to a rotator cuff disorder. Participants were predominantly seeking treatment for one shoulder. People who self-referred directly to the musculoskeletal service were also assessed for eligibility, as the typical route of referral varied across services. The participants did not routinely undergo diagnostic imaging, such as magnetic resonance imaging or ultrasound, as a requirement of the trial, as this is generally not recommended in primary care.

\section{Inclusion criteria}

- Men and women aged $\geq 18$ years.

- A new episode of shoulder pain (i.e. within the previous 6 months) attributable to a rotator cuff disorder (e.g. cuff tendonitis, impingement syndrome, tendinopathy or rotator cuff tear), using the diagnostic criteria set out in the BESS guidelines ${ }^{4}$ (see Appendix 1, Figure 13).

- Not currently receiving physiotherapy.

- Not being considered for surgery.

- Able to understand spoken and written English.

\section{Exclusion criteria}

- Participants with a history of recent significant shoulder trauma (e.g. dislocation, fracture or full-thickness tear requiring surgery).

- Those with a neurological disease affecting the shoulder.

- Those with other shoulder disorders (e.g. inflammatory arthritis, frozen shoulder or glenohumeral joint instability) or with red flags consistent with the criteria set out in the BESS guidelines. ${ }^{4}$

- Those who had received corticosteroid injection or physiotherapy for shoulder pain in the previous 6 months.

- Those with contraindications to corticosteroid injections.

\section{Recruitment}

\section{Recruitment of participants, screening and eligibility assessment}

Potentially eligible participants were identified by clinicians in NHS musculoskeletal services. People attended their clinic appointments in accordance with standard NHS procedures. The treating practitioner in the musculoskeletal services undertook a clinical assessment according to their usual practice. If a patient fulfilled the criteria for a rotator cuff disorder, they were assessed to see whether or not they met the GRASP trial eligibility criteria. Patients were provided with a copy of the participant information sheet and asked if they wished to be considered for the trial. Those who met the eligibility criteria and wanted to participate were approached for informed consent. Participants who did not meet the eligibility criteria or who did not wish to participate received standard NHS treatment. We recorded anonymous information on the age and sex of those who declined to participate so that we could assess the generalisability of those recruited. Reasons for declining were also recorded. 


\section{Informed consent and baseline assessment}

After participants had been assessed for eligibility, informed consent for participation in the GRASP trial was obtained by a research facilitator at the site who was trained in Good Clinical Practice (GCP). The following was explained to the participant: the exact nature of the study; what it would involve for the participant, including expectations that the participant would be willing and able to attend sessions to receive the study intervention; and any risks involved. The potential participant was provided with a patient information sheet and was given the opportunity to discuss issues and ask questions. In most cases, the process of obtaining informed consent took place during the initial musculoskeletal clinic appointment. Some participants required a second research appointment because they required more time to consider the study or because of local resources at site. Participants were then asked to complete a baseline assessment questionnaire that recorded simple demographic information and baseline measurements for the primary and secondary outcomes (Table 1).

TABLE 1 Outcomes measured and time points assessed

\begin{tabular}{|c|c|c|}
\hline Outcome & Measurement & Time point \\
\hline Demographic & $\begin{array}{l}\text { Age, sex, height, weight, ethnicity, marital status, smoking, date of } \\
\text { rotator cuff diagnosis, duration of symptoms, hand dominance, } \\
\text { affected shoulder, current work status, level of education, place } \\
\text { of residence, household income and state benefits }\end{array}$ & Baseline \\
\hline \multicolumn{3}{|l|}{ Primary } \\
\hline Pain and function & SPADI $^{10,11} 13$-item total scale & $\begin{array}{l}\text { Baseline, } 8 \text { weeks, } \\
6 \text { months, } 12 \text { months }\end{array}$ \\
\hline \multicolumn{3}{|l|}{ Secondary } \\
\hline Pain & SPADI ${ }^{10,11}$ five-item subscale & $\begin{array}{l}\text { Baseline, } 8 \text { weeks, } \\
6 \text { months, } 12 \text { months }\end{array}$ \\
\hline Function & SPADI ${ }^{10,11}$ eight-item subscale & $\begin{array}{l}\text { Baseline, } 8 \text { weeks, } \\
6 \text { months, } 12 \text { months }\end{array}$ \\
\hline Health-related quality life & EQ-5D-5L score 38 & $\begin{array}{l}\text { Baseline, } 8 \text { weeks, } \\
6 \text { months, } 12 \text { months }\end{array}$ \\
\hline Psychological factors & FABQ-PA five-item subscale ${ }^{39}$ and PSEQ- $2^{40}$ & $\begin{array}{l}\text { Baseline, } 8 \text { weeks, } \\
6 \text { months, } 12 \text { months }\end{array}$ \\
\hline Sleep disturbance & $|S|^{41}$ & $\begin{array}{l}\text { Baseline, } 8 \text { weeks, } \\
6 \text { months, } 12 \text { months }\end{array}$ \\
\hline GIT & Patient-rated Likert scale ${ }^{42}$ & $\begin{array}{l}8 \text { weeks, } 6 \text { months, } \\
12 \text { months }\end{array}$ \\
\hline RDA & $\begin{array}{l}\text { Patient-reported RDA, including work, social life and } \\
\text { sport activities }\end{array}$ & $\begin{array}{l}\text { Baseline, } 8 \text { weeks, } \\
6 \text { months, } 12 \text { months }\end{array}$ \\
\hline Exercise adherence & Patient-reported adherence to exercise & $\begin{array}{l}8 \text { weeks, } 6 \text { months, } \\
12 \text { months }\end{array}$ \\
\hline Medication usage & $\begin{array}{l}\text { Prescribed and over-the-counter medications, additional } \\
\text { steroid injection }\end{array}$ & $\begin{array}{l}8 \text { weeks, } 6 \text { months, } \\
12 \text { months }\end{array}$ \\
\hline Work disability & Sick leave (days) & $\begin{array}{l}8 \text { weeks, } 6 \text { months, } \\
12 \text { months }\end{array}$ \\
\hline Health-care use & $\begin{array}{l}\text { NHS outpatient and community services (e.g. GP, additional } \\
\text { physical therapy), NHS inpatient and day case (e.g. radiography, } \\
\text { MRI) and private health-care services }\end{array}$ & $\begin{array}{l}8 \text { weeks, } 6 \text { months, } \\
12 \text { months }\end{array}$ \\
\hline Out-of-pocket expenses & Patient-related out-of-pocket expenses recording form & $\begin{array}{l}8 \text { weeks, } 6 \text { months, } \\
12 \text { months }\end{array}$ \\
\hline
\end{tabular}

EQ-5D-5L, EuroQol 5 Dimensions, five-level version; FABQ-PA, Fear Avoidance Belief Questionnaire - Physical Activity; GIT, Global Impression of Treatment; ISI, Insomnia Severity Index; MRI, magnetic resonance imaging; PSEQ-2, Pain Self-Efficacy Questionnaire, two-item version. 


\section{Randomisation}

Consented participants were randomised to one of the four physiotherapy-led intervention groups $(1: 1: 1: 1)$ (see Trial design), using a centralised computer randomisation service RRAMP (Registration/ Randomisation and Management of Product; URL: https://rramp.octru.ox.ac.uk) provided by the Oxford Clinical Trials Research Unit (OCTRU). This was undertaken directly by the research facilitator at the site or by the research facilitator contacting the central randomisation centre by telephone, who then accessed the system on their behalf, depending on the facilities available at the study sites. Randomisation was computer generated and stratified by centre, age (18-35 years, > 35 years) and sex, using a variable block size to ensure that participants from each study site had an equal chance of receiving each intervention.

\section{Blinding}

Both the physiotherapists delivering the intervention and the study participants were informed of treatment allocation at the initial appointment. Because of the nature of the interventions being tested, it was not possible to blind them to the treatment allocation once treatment allocation was revealed. Where practical, team members were blinded until after data analysis was complete. Trial statisticians had access to treatment assignment during the study for the purposes of data monitoring and safety. Data entry personnel entered data from anonymised questionnaires, which included some details on treatments received.

\section{Interventions}

Full details of the exercise interventions are described in Chapter 3 and have been reported previously. ${ }^{43} \mathrm{~A}$ summary is provided here for continuity.

\section{Subacromial corticosteroid injection}

The subacromial corticosteroid injection was delivered prior to the progressive-exercise or best-practice advice intervention. The injections were predominantly carried out by extended-scope physiotherapists with appropriate post-registration qualifications in injection therapy who worked within a local patient group directive or as non-medical independent prescribers. ${ }^{34}$ This reflects an increasingly common practice in the NHS and ensured that the injections were delivered in the most cost-effective manner possible. The corticosteroid injection was given as per its marketing authorisation and in accordance with its normal indication and therapeutic dosage. ${ }^{44}$

The corticosteroid and local anaesthetic were given together in one injection or separately in two injections, depending on local treatment protocols at sites. The corticosteroid injected was either methylprednisolone acetate (Depo-Medrone ${ }^{\circledR}$, Pfizer Ltd, Walton Oaks, UK; up to $40 \mathrm{mg}$ ) or triamcinolone acetonide (Kenalog ${ }^{\mathrm{TM}}$, Bristol-Myers Squibb, Mulhuddart, Ireland; up to $40 \mathrm{mg}$ ), as per local treatment protocols. These are the two routinely injected corticosteroids for shoulder pain. There is no clear evidence that either corticosteroid is more effective than the other. ${ }^{30}$ The local anaesthetic was either $1 \%$ lidocaine (up to $5 \mathrm{ml}$ ) or $0.5 \%$ bupivacaine hydrochloride (up to $10 \mathrm{ml}$ ). We selected sites that adhered to these prescribing boundaries. The choice and dose of corticosteroid, local anaesthetic (including volume) and the injection site were recorded for each participant on a trial injection data collection form (see Appendix 2, Figure 14).

Participants were advised to take care and avoid heavy lifting for 24-48 hours after the injection. Appointments were co-ordinated so that participants typically received their injection within 10 days of randomisation. Very occasionally, a second injection was given after 6 weeks in accordance with the trial protocol (but within 16 weeks of the patient being randomised), but this injection was administered to only those patients who received good initial benefit from their first injection and who requested further pain relief to facilitate their exercises. Any participants who received a second injection had the dose, drug and date of administration recorded on a trial injection data collection form. 


\section{Progressive-exercise intervention}

Participants randomised to the progressive-exercise intervention received up to six individual faceto-face sessions with a physiotherapist over 16 weeks. These sessions included a behavioural component to encourage adherence to the exercises. A similar rationale has been used to good effect in other trials. ${ }^{21,45}$ We chose the number of sessions, spread over this time, to enable progression of the intensity of exercise and provide a sufficient amount of time for a physiological response in the neuromuscular system. ${ }^{46}$ Appointments were co-ordinated so that participants typically started their first exercise session within 14-28 days of randomisation, as per local appointment availability. The initial session lasted up to 60 minutes for assessment and setting up the home exercise programme, followed by up to five 20- to 30-minute follow-up sessions. Participants were provided with a folder containing an advice booklet, an exercise action planner and diary, and instructions on their exercise programme set up in collaboration with their physiotherapist. A resistance band was issued as required. The physiotherapists recorded the number of treatment sessions attended by each participant. The intervention was designed to support participants through a progressive dose of exercises and optimise adherence to the home exercise plan. The progressive-exercise programme was highly structured, but could be tailored to the needs and preferences of participants, with the aim of helping them achieve their rehabilitation goals. Importantly, the intervention could be delivered within the current NHS commissioning paradigm. ${ }^{47}$

\section{Best-practice advice intervention}

Participants randomised to the best-practice advice intervention received a single, individual faceto-face session with a physiotherapist, lasting up to 60 minutes. Again, appointments were co-ordinated so that participants typically started their exercise session within 14-28 days of randomisation, as per local appointment availability. After a comprehensive shoulder assessment, participants were given an advice booklet. The content of the advice in the booklet was the same as that provided for the progressiveexercise group, with the exception of the different exercise programme. An exercise diary was also provided, along with a simplified version of the exercise action planner (see Chapter 3). Tailored education, reassurance and self-management exercise advice, including advice on pain management and activity modification, was offered. Participants were also given a simple set of self-guided exercises, including at least one level of resistance band and an exercise video [available on a website and digital versatile disc (DVD)], which could be progressed and regressed, depending on their capability. The exercises were designed using similar concepts to those of the progressive-exercise intervention, such as increased resistance, but with a simpler range of exercise options that were not supervised.

The best-practice advice intervention was selected as the comparator because it is consistent with current clinical practice guidelines regarding the self-management advice that should be provided to people with rotator cuff disorders. ${ }^{4,5}$ In addition, people may find a single advice session and DVD preferential to a course of face-to-face physiotherapy sessions, as they do not have to come back to the hospital or clinic, take time off work or make carer arrangements.

\section{Concomitant care}

All participants were advised that they could take over-the-counter analgesia as required (e.g. paracetamol with or without codeine, or an oral non-steroidal anti-inflammatory drug) in accordance with the BESS guidelines. ${ }^{4}$ Participants could seek other forms of treatment during the follow-up period, but were informed that they should use usual routes (predominantly NHS referral) to do so. Additional treatments, including contact with their GP or other health professional, changes in medication, use of physical treatment and alternative therapies, were recorded as a treatment outcome through the patient questionnaires at 8 weeks, 6 months and 12 months post randomisation.

\section{Training and monitoring of intervention delivery}

All physiotherapists delivering study interventions, progressive exercise and best-practice advice had access to a comprehensive intervention manual and were required to have undertaken trial-specific training by a GRASP trial research physiotherapist. A rigorous quality control programme was also conducted to ensure intervention fidelity (see Chapter 3 ). 


\section{Outcomes}

\section{Primary outcome}

The primary outcome was shoulder pain and function over the 12 months post randomisation measured using the SPADI, ${ }^{10,11}$ which was developed to measure current (i.e. in the last week) shoulder pain and disability in an outpatient setting. The SPADI scale is based on 13 questions, all scored on a 0-10 scale, on which 10 is the worst score. In addition, the SPADI scale has a five-item pain subscale and an eight-item disability subscale. The subscale items are summed and converted to a 0-100 scale, where a higher value denotes more pain and/or disability. A systematic review of outcome measurement sets for shoulder pain trials showed that SPADI is the most commonly used measure to assess pain and disability. ${ }^{48}$ The SPADI scale has good psychometric properties, is used widely in the field and can be completed using a postal questionnaire.

\section{Secondary outcomes}

Secondary outcomes (see Table 1) were subdomains of the SPADI, which are pain measured using the SPADI five-item pain subscale ${ }^{10,11}$ and function measured using the SPADI eight-item disability subscale; ${ }^{10,11}$ health-related quality of life, measured using the well-validated EuroQol 5 Dimensions, five-level version (EQ-5D-5L) score; 38 psychological factors, measured using the Fear Avoidance Belief Questionnaire Physical Activity (FABQ-PA) five-item subscale ${ }^{39}$ and the Pain Self-Efficacy Questionnaire, two-item version (PSEQ-2);40 sleep disturbance, measured using the Insomnia Severity Index (ISI);41 patient global impression of change;42 RDA, including work, social life and sport activities; patient adherence to exercise; any serious adverse events (SAEs); health resource use, including consultation with primary and secondary care, prescribed and over-the-counter medication use, additional physiotherapy or injection use, and hospital admission; additional out-of-pocket expenses; and work absence (i.e. number of sickness days).

The EQ-5D-5 $\mathrm{L}^{38}$ is a validated, generic health-related quality-of-life measure comprising five dimensions, each with a five-level answer possibility and a health thermometer scale. The EQ-5D-5L can be used to report health-related quality of life in each of the five dimensions and each combination of answers can be converted into a health utility score, where 1 represents perfect health and 0 indicates health states equal to death. The health thermometer scale (EuroQol visual analogue scale) takes values between 0 and 100, where 0 represents worst imaginable health and 100 best imaginable health. It has good test-retest reliability and gives a single preference-based index value for health status that can be used for broader cost-effectiveness comparative purposes.

The Fear Avoidance Belief Questionnaire $(F A B Q)^{39}$ is a validated measure of fear-avoidance behaviour. The FABQ-PA is a subscale of the FABQ that measures fear-avoidance beliefs about physical activity using five items scored on a 0 (strongly disagree) to 6 (strongly agree) scale. The total score range for FABQ-PA is $0-24$, with higher scores representing greater levels of fear-avoidance behaviour.

The PSEQ ${ }^{40}$ is a well-established 10-item measure of pain self-efficacy (i.e. a belief in one's ability to carry out activities despite pain). PSEQ-2 is a two-item, short measure of pain self-efficacy. ${ }^{40}$ The two items reflect the confidence in ability to work and lead a normal life despite the pain, on a 0 (not at all confident) to 6 (completely confident) scoring scale. The total PSEQ-2 score is summed from the two items, giving a range from 0 to 12, with higher values representative of higher confidence levels despite the pain.

The ISI ${ }^{41}$ is a brief self-report measure of a patient's perception of their insomnia, targeting the subjective symptoms and consequences of insomnia, as well as the degree of concerns or distress caused by those difficulties. The ISI has seven items rated on a 0-4 scale and the total score is a summation of these 
items, with a value ranging from 0 to 28 . Higher scores are suggestive of more severe insomnia. A detailed interpretation of the ISI total score is as follows:

- A score of 0-7 indicates no clinically significant insomnia.

- A score of 8-14 indicates subthreshold insomnia.

- A score of 15-21 indicates clinical insomnia of moderate severity.

- A score of 22-28 indicates severe clinical insomnia.

The Global Impression of Treatment (GIT) ${ }^{42}$ is a simple method of measuring change in health status, with respect to shoulder problems, by charting self-assessed clinical progress on an 11-point scale that ranges from -5 (very much worse) to 5 (completely recovered). Psychometric properties include a minimum detectable change of 0.45 points and a minimally clinically important difference (MCID) of 2 points.

Return to desired activities is a self-reported outcome that aims to measure physical function during social life, recreational activities and work. RDA is an adapted version of the Disabilities of the Arm, Shoulder and Hand (QuickDASH), using three questions with a five-point Likert scale answer option, with lower scores indicating better function.

Other secondary outcomes (including medication usage, work disability, health-care use and out-of-pocket expenses) are analysed separately as part of a health economics analysis (see Chapter 5).

\section{Adverse events}

Expected adverse events occurring as a result of the trial intervention(s) were not recorded as part of the trial. Participants were provided with information on the potential adverse events resulting from exercise and corticosteroid injection (if applicable) as part of their treatment, including what they should do if they experienced an adverse event, as would happen as part of standard NHS procedures. SAEs (defined as any medical occurrence that could result in death, is life-threatening or results in hospitalisation or incapacity) were considered highly unlikely to occur as a result of either the exercise or the corticosteroid injection therapy delivered in this trial. However, if a SAE arose in the period from the participant's enrolment in the trial to their final visit for their allocated intervention, standard procedures for recording and reporting SAEs applied.

\section{Follow-up data collection}

Measurements for the primary and secondary outcomes were all patient reported and collected using postal questionnaires at 8 weeks, 6 months and 12 months after randomisation (see Table 1). Participants were asked to complete the questionnaire and return it to the GRASP study team in the prepaid envelope. For those who did not respond to the initial questionnaire, at least one postal reminder was sent. A web-based version of the questionnaire, and telephone and e-mail follow-up were used to contact those who did not respond to the postal questionnaire. Telephone and e-mail follow-up was also used to collect a core set of questionnaire items if these had not been fully completed on the returned questionnaire. To maximise response rates for the 12-month follow-up, a small monetary incentive (in the form of a gift voucher) was sent to all participants along with their 12-month follow-up questionnaire as a thank you for the time and effort involved. 


\section{Data management}

All data were processed according to the General Data Protection Regulation 2018 and all documents were stored safely in confidential conditions. ${ }^{49}$ All trial-specific documents, except for the signed consent form and follow-up contact details, referred to the participant using a unique study participant number/code and not by name. Participant identifiable data were stored separately from study data and in accordance with local procedures. All trial data were stored securely in offices that were accessible using a swipe card by the central co-ordinating team staff and authorised personnel only.

\section{Statistical methods}

\section{Sample size}

The target sample size for the trial was 704 randomised participants (176 in each treatment arm). This sample size was based on $90 \%$ power and $1 \%$ two-sided statistical significance to detect a minimally clinically important between-group difference of 8 points on the SPADI total scale,${ }^{10}$ assuming a baseline standard deviation (SD) of 24.3 (chosen as representative of the patient population ${ }^{50}$ ). This difference is the equivalent of a standardised effect size of 0.33 , which required a sample size of 550 participants [Power Analysis and Sample Size 13 NCSS Statistical Software Kaysville, UT, USA; URL: www.ncss.com (accessed 20 May 2021)]. Allowing for a potential loss to follow-up of $20 \%$ at 12 months inflated the sample size to 688. We further inflated the sample size to take into account the potential for a small clustering by physiotherapist effect in the progressive-exercise intervention group. We used an interclass correlation (ICC) of 0.001 , based on our experience with individually tailored physiotherapy interventions $\mathrm{s}^{51}$ and the expectation that each physiotherapist would treat approximately 20 participants in the progressiveexercise intervention group. This lead to an inflation of:

$$
f=1+(m-1) \times I C C=1+(20-1) \times 0.001=1.019
$$

and increased the sample size to the total of 704 participants.

This sample size was based on the assumption that there was no interaction effect and was powered for the two main effect comparisons: (1) progressive exercise compared with best-practice advice and (2) corticosteroid injection compared with no injection. However, this number of participants also provided $80 \%$ power and $5 \%$ two-sided significance to detect an interaction standardised effect size of 0.35 , if an interaction effect did exist. The interaction effect was tested before the main effect comparisons were undertaken. It should be noted that a non-significant interaction effect did not preclude a smaller interaction that this study was not powered to detect. We chose $90 \%$ power and $1 \%$ two-sided significance to provide more convincing evidence of any treatment effects discovered. No further adjustments to the sample size were made because of multiple testing. The Data Monitoring and Ethics Committee (DMEC) reviewed the sample size assumptions after 338 participants were recruited and no changes were made to the final sample size.

\section{Statistical analysis}

A separate $\mathrm{SAP}^{37}$ provides full details of all planned statistical analyses and was finalised prior to any primary outcome analysis. A summary is provided below.

The SAP was reviewed and received input from the TSC and DMEC. Any changes or deviations from the original SAP are described and justified in Chapter 4 and any additional publications, as appropriate. All statistical analyses were undertaken using Stata ${ }^{\circledR}$ (StataCorp LP, College Station, TX, USA). 
The primary statistical analysis was carried out on the basis of intention to treat (ITT), with all randomised participants included and analysed according to their allocated treatment group, irrespective of which treatment they actually received or their compliance with the proposed interventions. The two main effect comparisons for this $2 \times 2$ factorial trial were (1) progressive exercise compared with best-practice advice to determine the efficacy of progressive exercise [group $A$ (progressive exercise) + group $C$ (progressive exercise + injection) vs. group B (best-practice advice) + group D (best-practice advice + injection)] and (2) subacromial corticosteroid injection compared with no injection to determine the efficacy of subacromial corticosteroid injection ( $A+B$ vs. $C+D$ ). This 'factorial analysis' was conducted 'at the margins' of the table (Table 2). The sample size for this type of analysis was calculated under the assumption that there will be no intervention interaction effect (i.e. that progressive exercise would not interact with the steroid injection, such that it would work, work better or work worse only when used together rather than used alone). If a substantial interaction between the two interventions was present, the factorial analysis of the groups would lead to biased results and, therefore, the efficacy of each intervention would need to be drawn from comparisons within the intervention groups (i.e. 'inside-the-table' comparisons). Regardless of being able to detect a significant interaction effect, the results of the trial for the primary outcome, SPADI, meant that estimates were to be presented both 'inside the table' and 'at the margins', together with the size of the interaction. ${ }^{52}$ The success or otherwise of the interventions would be evaluated from the analysis results conducted based on evidence for presence/absence of a treatment interaction.

\section{Interaction}

An interaction between the two main effect comparisons was not expected, but the trial was powered to identify a moderate standardised interaction effect of 0.35 . The presence of an interaction between the two interventions was formally investigated before testing their effects on the primary outcome. An initial regression model was fitted for the primary outcome to predict the outcome of interest and included the two effects of interest [i.e. (1) individually tailored progressive-exercise programme compared with best-practice advice and (2) subacromial corticosteroid injection compared with no injection] and their interaction.

In the presence of a non-statistically significant treatment interaction (i.e. $p \geq 0.05$ ), a factorial analysis was planned to determine the success of the trial. The effects for the individually tailored progressiveexercise programme and corticosteroid injection were determined separately from this model as mean differences (MDs) with associated $99 \%$ confidence intervals (Cls), as appropriate, adjusted for the relevant covariates. The model used did not include an intervention interaction term.

If a statistically significant treatment interaction (i.e. $p<0.05$ ) had been detected then the effect of the individually tailored progressive-exercise programme and corticosteroid injection would have been evaluated from comparisons within the intervention groups, referred to as 'inside-the-table' comparisons (i.e. group A vs. group B to test the effect of the individually tailored progressive exercise programme and group $B$ vs. group $D$ to test for the effect of the corticosteroid injection) (see Table 2). The main effects and their interaction terms would have been included in the analysis model, their regression coefficient with corresponding $95 \% \mathrm{Cl}$ for the interaction terms presented and the reduced statistical power of this model noted.

TABLE 2 Factorial $2 \times 2$ analysis diagram

\begin{tabular}{llll} 
& No corticosteroid injection & Corticosteroid injection & $\begin{array}{l}\text { Effect of ProgEx } \\
\text { intervention }\end{array}$ \\
ProgEx intervention & Group A (ProgEx) & Group C (ProgEx + injection) & A + C vs. B + D \\
BPA intervention & Group B (BPA) & Group D (BPA + injection) & \\
Effect of corticosteroid injection & A + B vs C + D & & \\
\hline
\end{tabular}

BPA, best-practice advice; ProgEx, progressive exercise. 


\section{Primary outcome analysis}

The difference in SPADI between the two intervention groups was estimated overall and at each data collection time point using a repeated measures linear mixed-effects regression model. ${ }^{53}$ The model was adjusted for the fixed effects of age, sex and baseline SPADI, and random intercepts by centre and observations within participants. Robust standard errors (SEs) for treatment effects from all time points were reported. Clustering by physiotherapist in the progressive-exercise group was accounted for using cluster-robust SEs as part of the mixed-effects model. The final trial results were based on the adjusted model. A non-parametric statistical test (e.g. Mann-Whitney test for comparison of means) with no adjustment and medians and interquartile ranges (IQRs) were reported where approximate normality for the model residual terms was not established. Statistical significance was set at the $1 \%$ level and corresponding $99 \% \mathrm{Cls}$ were reported for the primary outcome. Flooring effects in the SPADI outcome over the 12 months were explored and reported for each treatment group.

\section{Secondary outcome analyses}

The intervention effects on secondary outcomes were analysed following the analysis method described for the primary outcome on the basis that the outcomes are clinically similar. If there was no statistically significant evidence of an interaction effect for the primary outcome, then no interaction effect would be assumed for the secondary outcomes. Likewise, if a statistically significant interaction effect was identified for the primary outcome, then the secondary outcomes would be analysed, assuming an interaction effect was present. Continuous secondary outcomes analyses were conducted following similar methods to the outline for the primary outcome analysis, using linear regression for continuous outcomes and logistic/ multinomial logistic regression (i.e. logit, mlogit or ologit, as appropriate) for binary and ordinal outcomes. Statistical significance for secondary outcomes was set at $5 \%$ and $95 \%$ Cls were reported.

\section{Missing data}

Missing data were reported and summarised by treatment group. Item-level imputation for the primary outcome SPADI was carried out for items where no more than two out of five items in the pain subscale were missing and no more than three out of eight items in the function subscale were missing, given that no more than $10 \%$ of cases had missing data. 54,55 Missing continuous primary and secondary outcomes were handled as part of the likelihood-based estimation of the repeated measures mixed-effects model, assuming the data were missing at random. ${ }^{56}$ This method took account of missing observations owing to missed visits or a participant leaving the study prematurely. The distribution of missing data was explored to assess the assumption of data being missing at random. Full details are provided in the SAP. ${ }^{37}$

\section{Sensitivity analysis}

Sensitivity analyses were used to assess the robustness of the trial results in the light of the assumptions made about the underlying missing data mechanism. Most analyses assume data to be missing at random or missing completely at random. The sensitivity analysis, therefore, assumed missing not at random, such that missing outcomes were assumed to be worse or better than the observed outcomes.

\section{Prespecified subgroup analysis}

Subgroup effects in the following prespecified subgroups were analysed for the primary outcome, utilising subgroup-by-treatment interactions:

- Age: $\leq 64$ years vs. $\geq 65$ years. (Rationale: increasing age has been shown to be associated with poorer outcome. ${ }^{57,58)}$

- Sex: male vs. female. (Rationale: prevalence is higher in males than in females. ${ }^{1}$ )

- Smoking status: never smoked vs. former smoker or current smoker. (Rationale: smoking has been shown to be associated with a negative effect on tendon healing. ${ }^{59}$ ) 
- Higher SPADI score at baseline. (Rationale: higher pain and functional disability at baseline may be associated with poorer outcome. We defined a higher SPADI score as $\geq 50$ at baseline when the SPADI is converted to the 0 to 100 scale. ${ }^{19,57}$ )

- Higher pain self-efficacy (PSEQ-2) score at baseline. (Rationale: higher belief in one's ability to carry out activities despite pain may be associated with better outcome. We define a higher PSEQ-2 score as $\geq 8$ at baseline when the PSEQ- 2 is converted to the $0-12$ scale.)

\section{Supplementary/additional analyses}

A complier-average causal effect (CACE) analysis was used to investigate the role of compliance in the treatment effect, given the CACE assumptions described in the SAP. Compliance with intervention was defined in the $\mathrm{SAP}^{37}$ as follows. For the progressive exercise intervention, participants were considered compliant with treatment if they had been signed off for completing treatment or if they receive all six physiotherapy sessions. For corticosteroid injection intervention, participants were considered compliant if they received at least one injection. If no evidence of a statistically significant interaction between the two treatments was identified, then this analysis was planned to be conducted 'at the margins' and using a similar mixed-effects model. If a statistically significant treatment interaction for the primary outcome was identified, the CACE analysis would be conducted 'inside the table'.

\section{Cost-effectiveness analysis}

An economic evaluation was integrated within the GRASP trial design. Full details are described in Chapter 5 and a summary is described here for continuity.

The economic evaluation, in the form of a cost-utility analysis, was conducted from the recommended NHS and Personal Social Services (PSS) perspective. .00 Individual patient data on the use of health and social services were collected at 8 weeks, 6 months and 12 months post randomisation as part of the follow-up data collection process (see Table 1). The cost of delivering each intervention, including physiotherapists' training, materials, delivery of the progressive exercise and advice sessions, and corticosteroid injections, were also estimated. Participants' health-related quality of life was captured through the EQ-5D-5L at baseline and at 8 weeks, 6 months and 12 months post randomisation.

\section{Patient and public involvement}

Patient and public involvement (PPI) was central to the design of the GRASP trial and was maintained throughout the trial set-up, implementation and dissemination. PPI representatives were involved in a number of ways. First, as part of the initial trial design, we held a PPI study development meeting, supported by the Research Design Service South Central (Oxford, UK), where the proposed research was presented and discussed with attendees. The views expressed by the patients contributed to the trial design and subsequent trial protocol. In particular, an outcome looking at sleep disturbance was included, as this was deemed to be very important to patients. The PPI representatives also advised keeping the number of self-reported outcomes to a minimum to avoid an undue burden on the participants and recommended using diaries so that the patients could record and monitor their own progress.

Patient and public involvement representatives also attended the GRASP intervention development meeting (see Chapter 3) and provided valuable practical input regarding delivery and acceptability of the progressive-exercise and best-practice advice interventions. In addition, PPI representatives were involved in reviewing the participant information sheets and participant questionnaires to ensure that they were accessible and user-friendly. Two PPI representatives were formal members of the GRASP TSC and attended and actively contributed to these meetings, including the final results meeting where data were shared and interpreted. As part of the dissemination process, the PPI representatives also reviewed and advised on the wording of the letter to trial participants, advising them of the GRASP trial results. 


\section{Ethics approval and monitoring}

\section{Ethics committee approval}

The GRASP trial protocol and all related documentation (e.g. informed consent forms, participant information leaflets, patient questionnaires and any proposed advertising material) was approved by the Berkshire B Research Ethics Committee (reference 16/SC/0508) and Integrated Research Application System (ID 199243). The trial was also approved by the UK competent authority the Medicines and Healthcare products Regulatory Agency, as it was classified as a clinical trial of an investigational medicinal product (EudraCT number 2016-002991-28). The trial was conducted in accordance with the principles of the Declaration of Helsinki ${ }^{61}$ and the Medical Research Council's GCP guidelines. ${ }^{62}$

\section{Trial Management Group}

A Trial Management Group, consisting of the core trial team, chief investigator and co-applicants, was responsible for the day-to-day running of the trial and met monthly to report on progress and ensure that milestones were met. A trial manager oversaw all aspects of the day-to-day trial management.

\section{Trial Steering Committee}

A TSC was responsible for monitoring the trial's progress and providing independent advice. The TSC comprised an independent clinician, two specialist physiotherapists, a statistician, a health economist and two patient representatives.

\section{Data Monitoring and Ethics Committee}

A DMEC was responsible for monitoring the trial's progress and providing independent advice. It advised the chairperson of the TSC if, at any time, in its view, the trial should be stopped for ethics reasons, including concerns about participant safety. The DMEC comprised an independent clinician, health service researchers, a specialist physiotherapist and a statistician. 


\section{Chapter 3 Intervention description and rationale}

Darts of this chapter have been reproduced with permission from Keene et al. ${ }^{43}$ This is an Open Access article distributed in accordance with the terms of the Creative Commons Attribution (CC BY 4.0) license, which permits others to distribute, remix, adapt and build upon this work, for commercial use, provided the original work is properly cited. See: http://creativecommons.org/licenses/ by/4.0/. The text below includes minor additions and formatting changes to the original text.

\section{Developing the GRASP trial exercise interventions}

The GRASP trial interventions were developed using Medical Research Council guidance for developing and evaluating complex interventions. ${ }^{63}$ We took into account clinical guidelines, research evidence, current practice variation, deliverability in the NHS (in terms of staffing, resources and time), expert and patient opinion, acceptability to clinicians and patients, and the need to ensure consistency in delivery and reproducibility. The intervention development and descriptions have been reported previously. ${ }_{4}^{43}$

\section{Intervention development}

In Chapter 1, the lack of evidence for particular exercises, treatment intensities or durations were outlined. With no clear evidence or clinical consensus, advice from clinicians, patient and public representatives and other experts was crucial to the development of the GRASP trial interventions. Twenty-six clinicians, researchers, patients and public representatives attended a GRASP intervention development meeting (June 2016). Delegates discussed and evaluated a comprehensive list of 22 exercise types commonly used in clinical practice and/or reported in trials of shoulder pain treatments (see Appendix 3, Table 26). The delegates categorised the exercises into essential exercises, optional exercises or exercises considered not important for managing rotator cuff disorders.

Those selected as essential exercises generally strengthen the posterior rotator cuff muscles (i.e. supraspinatus, infraspinatus and teres minor) and load the shoulder into an elevated position, consistent with current trial evidence. ${ }^{17,64}$ The GRASP trial, therefore, included these exercise types as an essential component of both the progressive-exercise and best-practice advice exercise interventions. Delegates agreed that both exercise interventions should be as practical and simple as possible. Some exercises were considered important for patients with specific presentations or in particular circumstances. These were included as optional exercises in the progressive-exercise intervention. They were not incorporated into the best-practice advice intervention, which prioritised a simple, progressive set of exercises that were likely to benefit most patients and could be easily understood and performed at home without supervision.

Delegates agreed that stretching was not a priority target in rotator cuff disorders and that range of motion could be incorporated into other active exercises. A posterior capsule/soft tissue stretch for the shoulder was included as an option in the progressive-exercise intervention as we recognised that these stretches feature in many exercise programmes evaluated in other clinical trials. ${ }^{14}$ Isolated exercises to correct posture towards a theoretical ideal were not included, as there is limited evidence for this approach. ${ }^{65}$ Experts disagree on whether exercises can or should provoke symptoms or should be symptom-free. There is limited evidence for these alternatives, although evidence is building for the acceptability of mild-to-moderate pain symptoms during exercise. ${ }^{66,67}$ The participant information booklet, which was included as part of the GRASP trial intervention materials, and treating physiotherapists advised participants that some pain during the exercises is acceptable, provided that they found it manageable and symptoms resolved to an acceptable level within a few hours. 
After the meeting, we developed detailed intervention manuals for the treating physiotherapists that described the key components of the progressive-exercise and best-practice advice interventions. We also developed patient-facing materials, which were reviewed by patient representatives.

\section{Internal pilot}

As part of the GRASP trial, we ran an internal pilot from February to June 2017 at three sites, recruiting 42 participants. One of the aims of the internal pilot was to test and refine the recruitment process and explore treatment acceptability. The physiotherapists delivering the GRASP exercise interventions were also asked to provide feedback on the delivery of the intervention. The exercise intervention manuals and training materials were subsequently refined to clarify identified misconceptions. The interventions did not require significant modifications.

\section{The GRASP trial interventions}

Table 3 summarises the key components of the GRASP exercise interventions. The interventions were delivered face to face and one to one by UK-registered physiotherapists based at physiotherapy and musculoskeletal services across England.

TABLE 3 Overview of GRASP trial exercise interventions, as per Template for Intervention Description and Replication criteria

\begin{tabular}{|c|c|c|}
\hline TIDieR items ${ }^{68}$ & Progressive exercise & Best-practice advice \\
\hline Brief name & \multicolumn{2}{|l|}{ GRASP } \\
\hline Why & \multicolumn{2}{|c|}{$\begin{array}{l}\text { Physiotherapy-led exercise and advice are commonly used to manage rotator cuff disorders; } \\
\text { however, evidence is lacking in terms of how exactly it should be delivered and whether or } \\
\text { not patients do better if they receive a corticosteroid injection before starting an exercise } \\
\text { programme }\end{array}$} \\
\hline What & $\begin{array}{l}\text { Home exercise and advice programme } \\
\text { overseen by physiotherapist over six or fewer } \\
\text { sessions within } 16 \text { weeks }\end{array}$ & $\begin{array}{l}\text { Home exercise and advice programme } \\
\text { initiated during a single face-to-face session } \\
\text { with a physiotherapist, and then performed } \\
\text { unsupervised by participant at home }\end{array}$ \\
\hline Materials: participants & $\begin{array}{l}\text { Participant information booklet (see Table 4) } \\
\text { and exercise instruction sheets with photos } \\
\text { Action planner and exercise diary } \\
\text { (see Appendix 3, Figure 15) } \\
\text { Resistance bands (if applicable) }\end{array}$ & $\begin{array}{l}\text { Participant information booklet } \\
\text { (see Table 4), incorporating exercise } \\
\text { instructions with photos } \\
\text { Action planner and exercise diary } \\
\text { (see Appendix 3, Figure 22) } \\
\text { Resistance bands } \\
\text { Exercise instruction videos available on } \\
\text { website or DVD }\end{array}$ \\
\hline $\begin{array}{l}\text { Materials: } \\
\text { physiotherapists }\end{array}$ & $\begin{array}{l}\text { Therapist manuals detailing all aspects of the } \\
\text { trial and the progressive-exercise intervention, } \\
\text { and a quick reference guide for use in the } \\
\text { clinic } \\
\text { Training, including } 1 \text { full day (at least } \\
4-5 \text { hours) of face-to-face training delivered } \\
\text { by GRASP trial research physiotherapists }\end{array}$ & $\begin{array}{l}\text { A therapist manual detailing all aspects } \\
\text { of the trial and the best-practice advice } \\
\text { intervention } \\
\text { At least half a day of training }\end{array}$ \\
\hline Procedures & $\begin{array}{l}\text { Initial appointment } \\
\text { Assess participant as per normal } \\
\text { physiotherapy practice }\end{array}$ & $\begin{array}{l}\text { Single face-to-face appointment } \\
\text { Assess participant as per normal } \\
\text { physiotherapy practice }\end{array}$ \\
\hline
\end{tabular}


TABLE 3 Overview of GRASP trial exercise interventions, as per Template for Intervention Description and Replication criteria (continued)

\section{TIDieR items ${ }^{68}$}

\section{Who provides}

How

Where

\section{Progressive exercise}

Issue folder containing the progressiveexercise participant information booklet

Agree level of exercise that is most appropriate for the participant initially (see Figures 1 and 2)

Advice should address barriers to exercise identified during assessment

Provide education regarding pain during and after exercise

Help participant to complete exercise documentation (i.e. the exercise diary and action planner)

Make follow-up appointment(s)

Complete treatment log

Appointments 2-6

Reassess as per normal physiotherapy practice

Assist participant to progress/regress exercises

Reassure the participant and reinforce key messages from the advice and education

Review home exercise programme using the exercise diary

Discuss return to functional activities

Review action planner

Complete treatment log (after every session)

Physiotherapists already working in NHS musculoskeletal services who have attended the progressive-exercise intervention training

The GRASP trial does not exclude physiotherapists based on number of years qualified or experience in treating shoulder conditions

Participants receive up to six sessions with a physiotherapist over 16 weeks

The initial session lasts up to 60 minutes for assessment. It is then followed by up to five 20- to 30-minute sessions

Physiotherapy sessions are in outpatien clinics based in the NHS

The exercise programme is performed by the participant at home

\section{Best-practice advice}

Issue best-practice advice participant information booklet

Explain exercise ladder (see Appendix 3, Figure 21) and agree with participant what level of the ladder is most appropriate initially

Educate participants on how to progress and regress their exercises

Ensure that the participant knows how to access the exercise videos online or issue DVD (or both)

Advice should address barriers to exercise identified during assessment

Provide education regarding pain during and after exercise

Help participant to complete exercise documentation (i.e. the exercise diary and action planner)

Strategies to encourage adherence to exercise were less extensive than in the progressive-exercise intervention, as they need to be feasible to deliver within a single session

Discharge participant with advice/ encouragement to continue with the selfmanagement exercise programme for at least 16 weeks

Complete treatment log

Physiotherapists already working in NHS musculoskeletal services who have attended the best-practice advice intervention training

The GRASP trial does not exclude physiotherapists based on number of years qualified or experience treating shoulder conditions

Participants receive a single face-to-face session with a physiotherapist lasting up to 60 minutes

Same as progressive-exercise intervention 
TABLE 3 Overview of GRASP trial exercise interventions, as per Template for Intervention Description and Replication criteria (continued)

\section{TIDieR items ${ }^{68}$}

When and how much

Tailoring

\section{Intervention fidelity}

How well: training

How well: physiotherapists

How well: participants

Physiotherapists review the exercise diary at each subsequent session

Participants asked to report exercise frequency in postal follow-up questionnaires at 8 weeks, 6 months and 12 months
14-28 days of randomisation, as per local appointment availability

Up to five follow-up sessions arranged within 16 weeks

Can be fewer than six sessions if participant has met rehabilitation goals and is selfmanaging condition

Volume of exercise described in Figure 2

Education and advice

Focus of education and advice are individualised and based on assessment

\section{Exercises}

Selection, manipulation of sets, repetitions and/or load is a joint decision-making process

Range of motion and position may be modified to accommodate the patient's comfort and preferences comprehensive manuals completed anonymously with the Trial Management Team and the site

\section{Best-practice advice}

Same as progressive exercise, but without any follow-up appointments

\section{Education and advice}

Focus of education and advice are individualised and based on assessment

\section{Exercises}

The range of motion through which an exercise is performed, and the load and volume, may be increased or decreased

(he pilot phase and contained all key operational procedures for each intervention to supplement the

All aspects of training delivery, content, structure, duration and therapists' confidence to implement the intervention were evaluated using the post-training feedback forms that were

Intervention fidelity is monitored centrally using treatment logs and during site monitoring and quality assurance visits. If performance is found to be below the required standard of protocol adherence, further measures (e.g. further training) will be instituted after discussion

How well: reporting Intervention fidelity is reported with main trial results

Research physiotherapists from the GRASP trial team provided 1 full day (at least 4-5 hours) of faceto-face training on the progressive-exercise intervention and at least half a day of face-to-face training on the best-practice advice intervention to the relevant delivering physiotherapists. Details on who provided the interventions are provided in Chapter 4. Physiotherapists were trained to deliver either the progressive-exercise intervention or the best-practice advice intervention (not both) to reduce the risk of contamination. Training included the trial background, how to deliver the intervention, 
the exercises permitted within the trial protocol, behavioural techniques to improve adherence, trial reporting and paperwork, and practical examples using case studies. Physiotherapist manuals were provided, which detailed all aspects of the trial and interventions. GRASP trial team members were available post training to provide support and answer queries.

\section{GRASP trial exercise interventions}

\section{Assessment and advice}

Appointments were co-ordinated so that participants typically started their first exercise session within 14-28 days of randomisation. The physiotherapist carried out an initial assessment, as per their usual practice, to identify which shoulder movements, in relation to the rotator cuff, were particularly problematic in terms of pain, weakness and restriction, and the associated functional deficits. All GRASP trial participants received a participant information booklet, which contained education and advice relevant to people with a rotator cuff disorder (Table 4). All parts of the booklet contained the same information in the progressive-exercise and best-practice advice intervention groups, with the exception of the specifics of the exercise guidance. Advice included use of over-the-counter analgesia, as per BESS guidance. ${ }^{69}$ The treating physiotherapist reinforced the education and advice aspects relevant to that participant. All participants were advised to perform the exercises for at least 4 months and to continue to do so for longer if they were still improving or finding them helpful. Physiotherapists delivering the interventions were trained in questioning techniques based on cognitive-behavioural models ${ }^{70}$ to elicit and address unhelpful beliefs about shoulder pain or exercise that can impede exercise adherence. ${ }^{71}$

TABLE 4 Contents of the GRASP trial participant information booklet

\section{Section}

Introduction

What is the rotator cuff?

What does the rotator cuff do?

What can go wrong with the rotator cuff?

What can I do to help my rotator cuff problem?

\section{Maintaining physical fitness}

Return to usual physical activities

Modifying activities

Work

Sleeping

Looking after your mental well-being

Coping with flare-ups

Exercise guide

\section{Content}

Summary of common treatments for rotator cuff disorders

Description of rotator cuff muscles

Function of rotator cuff muscles

Possible mechanisms and common symptoms of rotator cuff disorders

Introduction to role of self-management advice, includes simple pain education, pain management (including use of heat and cold, medication and pacing)

Importance of staying active

Fear avoidance and deconditioning, and how to address these

Modifying rather than avoiding activities

Advice on returning to work activities

Sleep positions, pacing to lessen night-time pain

Activities to maintain a positive mood and when to seek medical advice

Planning exercise and activity for times when pain is more severe

Benefits of exercise and how long to continue with exercise

Pain during and after exercise

Guidance on doing GRASP trial exercises with full instructions (e.g. colour photographs and text explaining technique and modifications that can be made)

Note

Exercise details were specific to the progressive-exercise or best-practice advice intervention. 


\section{Progressive-exercise intervention}

Participants received up to six sessions with a physiotherapist over 16 weeks. The initial session was set to last up to 60 minutes for assessment and treatment initiation, followed by up to five 20- to 30-minute follow-up sessions. The physiotherapist and participant decided how frequently to schedule the review appointments within the 16-week time frame. Six sessions over 16 weeks was set to allow the exercise intensity to be progressed and because it was a volume of physiotherapy within the range of feasible delivery in the NHS. It was also deemed to be a sufficient time for a physiological response in the neuromuscular system. ${ }^{46}$ The participant information booklet was provided in a file so that instructions on the progressive exercises selected could be inserted, including detailed guidance and photos for each exercise. The physiotherapist and participant jointly chose one to three exercises to address the problems identified during the assessment (Figure 1). We anticipated participants presenting with different problems and pain irritability and, therefore, the exercises were categorised into three difficulty levels (level 1, 2 and 3 exercises), with progressions within each level and different aims and guidelines to match indications for use.



FIGURE 1 Process map of the GRASP trial progressive exercise intervention. 


\section{Level 1 exercises: simple shoulder movements}

Level 1 exercises aimed to reduce fear, encourage normal movement, improve shoulder mobility and build confidence in exercising independently at home (see Appendix 3, Figure 17). The exercises were considered appropriate for participants with irritable and/or severe shoulder pain and/or fear avoidance. The frequency and the number of sets and repetitions for each exercise were agreed between the physiotherapist and participant. Depending on symptom severity, it was recommended that some participants could reasonably move straight to level 2 exercises.

\section{Level 2 exercises: progressive structured resistance training}

Level 2 exercises included the essential exercises that target strengthening of the posterior rotator cuff muscles and other optional exercises (see Appendix 3, Figure 18). The essential exercises focused on movements commonly affected by a rotator cuff disorder (i.e. resisted external rotation, flexion and abduction of the shoulder). ${ }^{64}$ Resistance exercises for other shoulder movements were optional. If participants were prescribed exercises at level 2 , at least one had to be from the essential exercise category.

The American College of Sports Medicine guidance for progressive resistance training recommends two to three sessions of resistance training per week, ${ }^{20}$ whereas many studies of resistance training in patients with musculoskeletal disorders use daily exercise programmes.. ${ }^{21,72} \mathrm{We}$ attempted to strike a balance, ensuring that the resistance training was effective, but also regular enough to address other aims, such as building confidence in moving the arm and re-learning motor skills. We asked GRASP trial participants to do their exercises 5 days per week, with 2 non-consecutive recovery days.

Physiotherapists were taught to regulate the exercise intensity using the modified Borg scale of perceived exertion, an 11-point version of the Rating of Perceived Exertion (RPE) scale, ${ }^{73}$ validated for quantifying the intensity of resistance exercise. ${ }^{74}$ Participants started at a moderate load ( 3 or 4 on the Borg scale) to enhance motivation and adherence and reduce the likelihood of symptom flare-up. Figure 2 contains detailed guidance on the scale and how level 2 exercises were initiated, progressed and regressed. Where appropriate, resistance bands or hand weights were incorporated into the exercise, with the level of resistance recommended being guided by the RPE scale feedback from the participant.

\section{Level 3 exercise: patient-specific functional restoration}

It was recommended that participants progress to level 3 when their initial problems (e.g. weakness) began to resolve. The physiotherapist altered the exercise programme to be more task specific (e.g. returning to sport) by devising a new exercise in consultation with the participant. Level 3 exercises aimed to modify the essential resistance-training exercises towards the specific movements required to achieve the participant's individual functional goals. The exercise could be high or low load, using many or few repetitions, depending on the participant's needs. It was recognised that not all GRASP trial participants would reach or need this stage of the programme. We anticipated that level 3 exercise instructions would be given face to face and reinforced with written guidance to aid recall.

\section{Optional stretching exercise}

Posterior capsule and/or soft tissue stretches of the shoulder (see Appendix 3, Figure 19) were included as optional exercises at any stage. We recommend selective use, generally for younger adults engaged in throwing or other overhead athletic or physical activities ${ }^{75}$ who have posterior capsule tightness. We anticipated these exercises to be suitable for participants with low irritability and if they did not provoke symptoms. Although discomfort and stretching sensations were considered acceptable during stretches, we did not anticipate provocation of anterior shoulder pain or reproduction of the participant's specific symptoms. We advised holding stretches for $20-30$ seconds, but the physiotherapist and participant decided the number of repetitions and frequency. 
(a)

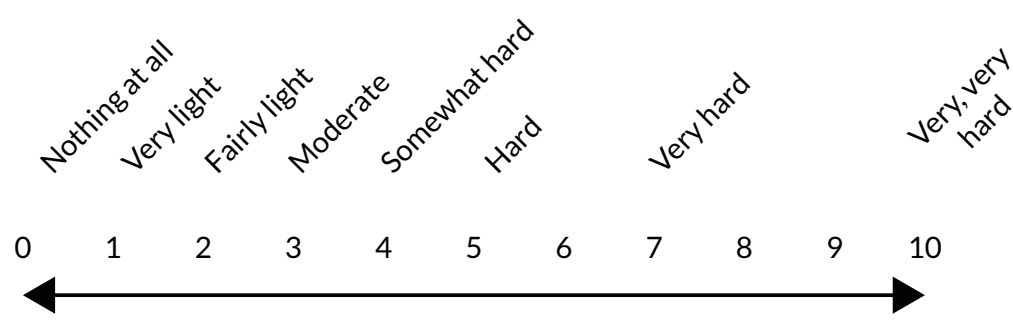

Using a number between 0 and 10, indicate on the scale above how hard it is to do the exercise. Use the descriptions above the scale to help you

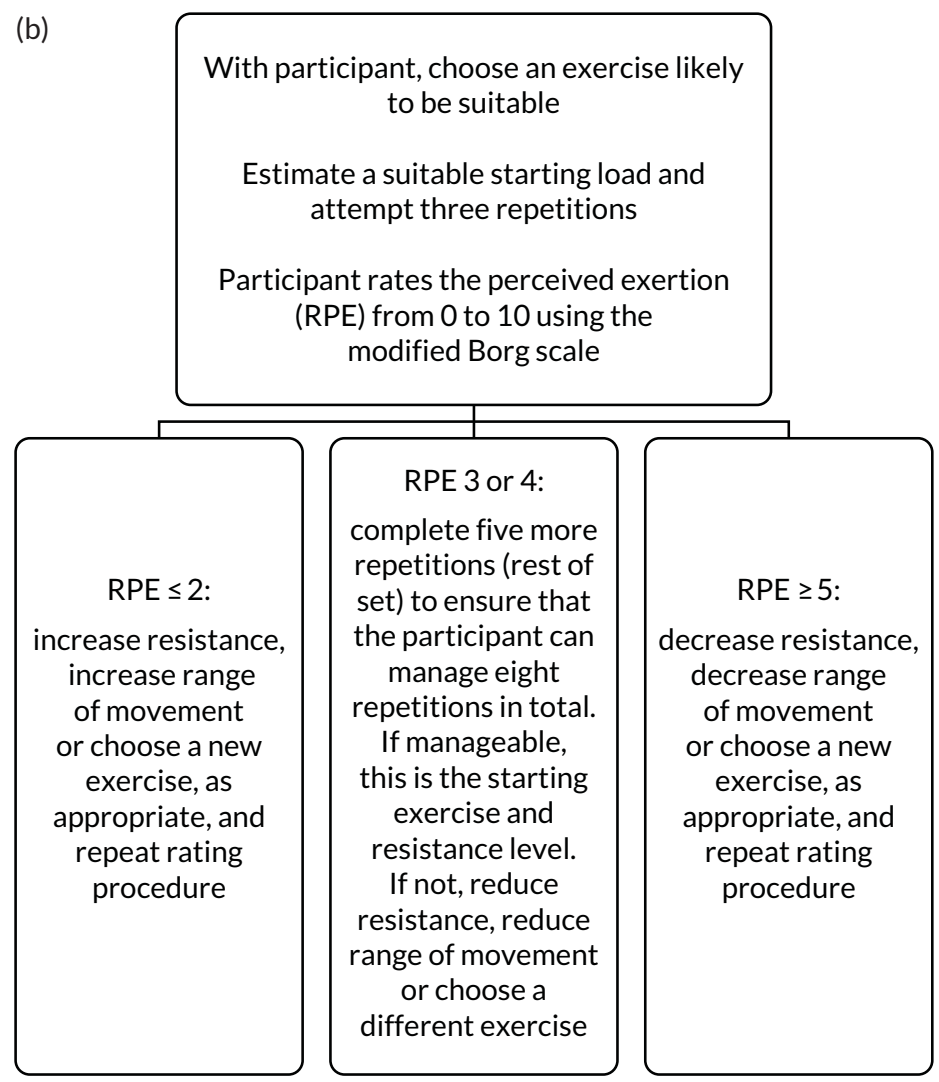

(c)

Initial

Once RPE is at 3 or 4 , start home exercise with one set of eight repetitions (advising to build up to 12 repetitions as appropriate), once per day, five times per week (with 2 non-consecutive rest days in between)

Repeat for each level 2 exercise in programme up to maximum of three exercises; advise 2 minutes' rest between exercises (and sets)

\section{Progression}

If RPE lower than 3 and has successfully progressed to 12 repetitions, exercises may be progressed:

- Add sets up to two or three sets of 8-12 repetitions; rest 2 minutes between sets

- Increase load/resistance to get back to RPE 3 or 4

- Further increase load/resistance to RPE 4-5, or

$5-7$, if participant is confident

- Increase in sets and load could be done together if the participant and therapist agree

A more difficult exercise should be considered when either:

- The maximum volume of sets and repetitions $(3 \times 12)$ at Borg RPE $=7$ is achieved

- If change of exercise is deemed appropriate to progress the exercise programme

\section{Regression}

If the patient cannot do eight repetitions of a particular exercise at the selected load and target RPE, or can manage this but experiences an unacceptable increase in pain, reduce the load to a level at which they are able to do at least eight repetitions

If they are still unable to do one set of eight repetitions using the lowest load, then you can:

- Remove load altogether

- Reduce repetitions to fewer than eight

- Reduce range of movement or alter the position

- Choose another exercise

FIGURE 2 Guidance for setting level 2 resistance exercises in the GRASP trial. (a) Modified Borg scale; (b) setting the initial exercise; and (c) progression and regression decision guidance. 


\section{Exercise progression}

During the physiotherapist training, emphasis was placed on the need to progress the exercise interventions as much as possible. To determine that this had in fact occurred, treatment logs completed for each session by the physiotherapists [detailing prescribed exercises, number of sets/repetitions (i.e. volume) and exercise intensity ${ }^{73}$ ] were analysed to allow an estimation of treatment progression for the progressiveexercise intervention (see Chapter 4). Exercises were categorised and ranked in order of difficulty level ( 1 = least difficult, $8=$ most difficult) according to the following scale:

1. passive movements

2. isometric

3. active assisted

4. active (unassisted)

5. loaded exercises (isotonic) in neutral $\left(0^{\circ}\right)$

6. loaded exercises (isotonic) at $90^{\circ}$ (supported)

7. as for 6 , but unsupported

8. as for 7 , but through range.

Progression of the progressive-exercise intervention was defined as an increase in exercise difficulty, or volume and/or load across attended sessions. Maintained indicates no changes in exercise parameters. Regressed is defined as a decrease in exercise level, or volume and/or load.

\section{Best-practice advice intervention}

The best-practice advice intervention was a single face-to-face session with a physiotherapist, lasting up to 60 minutes. With only one session, there was a substantially greater reliance on self-management than in the progressive-exercise intervention. As per the progressive-exercise intervention, participants were given a participant information booklet and the treating physiotherapist reinforced the education and advice aspects that were particularly relevant.

Participants were given a simple set of self-guided exercises, including at least one level of resistance band, to improve shoulder strength and function, which could be progressed and regressed independently at home, depending on their capability. The exercise instructions were given in the participant information booklet and in exercise videos available on the internet and DVDs. We aimed to make the information accessible and appealing to a wide range of individuals by using different media. ${ }^{76}$

This intervention offered a simpler range of exercise options than the progressive-exercise intervention, although the underlying principles remained the same. The physiotherapist and participant chose one or two exercises from the best-practice advice exercise ladder together (see Appendix 3, Figure 21). The exercises on the ladder were arranged with easier exercises on the lower rungs and more difficult exercises on the higher rungs. Participants were recommended to start at the level of exercise that they were capable of undertaking, not necessarily the lowest rung. If two exercises were selected, then these did not need to be from the same rung. The exercises at the lowest level were simple shoulder exercises to reduce fear of movement, encourage normal movement, build shoulder mobility and build confidence in carrying out self-directed exercises. Higher levels introduced a greater extent of resistance exercises for posterior rotator cuff strengthening.

The Borg RPE scale was not used to measure intensity for participants in the best-practice advice group to simplify the programme and maximise independent participant progression. We advised participants that they should find the exercise(s) moderately difficult (i.e. not easy but not extremely hard). They began with one set of eight repetitions at the selected load and aimed to build up to 12 repetitions, exercising once daily for 5 days per week, with 2 non-consecutive rest days. If the exercise(s) became too easy, another set could be added (up to a maximum of three sets). If the expanded set became too easy, the advice was that the exercise(s) should be exchanged for the next level of difficulty. 
The next exercise, again, started with one set of eight repetitions and built up in the same way. If the participant encountered difficulties, they were advised to reduce the load and/or number of sets/repetitions or regress to an easier exercise.

\section{Strategies to encourage exercise adherence}

Estimates suggest that up to $70 \%$ of patients do not adhere to prescribed physiotherapy treatments. ${ }^{25}$ As part of the GRASP trial interventions, we, therefore, incorporated strategies to promote adherence to the exercise interventions. These strategies were less extensive in the best-practice advice intervention than in the progressive-exercise intervention, as they needed to be feasibly delivered within a single session (see Appendix 3, Table 27).

Modifiable behavioural targets were identified from a systematic review of barriers to physiotherapy adherence, including in-treatment exercise adherence, low self-efficacy, depression, anxiety, helplessness, greater perceived barriers to exercise and pain levels during exercise. ${ }^{24}$ For a summary of the behaviour change techniques used in the GRASP exercise interventions, based on the behaviour change techniques taxonomy, ${ }^{77}$ see Appendix 3, Table 27. These techniques were selected based on their evidence base, ${ }^{78}$ successful use in other trials ${ }^{72}$ and recommendations in the NHS Health Trainer Handbook. ${ }^{79}$ Participants were asked to complete an exercise action planner and exercise diary, which were tailored to the interventions (see Appendix 3, Figures 15, 22 and 23). Although exercise diaries have questionable reliability for measuring adherence because of issues with real-time compliance and recall bias, ${ }^{80}$ there is evidence to suggest that they promote adherence. ${ }^{81}$ We assessed adherence to exercises in the follow-up questionnaires, in which participants reported exercise frequency.

\section{Monitoring, quality assurance and safety}

We monitored intervention delivery fidelity using site monitoring visits, quality assurance visits and central monitoring of treatment session logs. Each site was visited annually to ensure that the physiotherapists adhered to the intervention protocol for each exercise arm. A member of the GRASP trial research team attended an appointment session for each trial intervention and completed a standardised checklist covering knowledge of trial procedures, returning trial paperwork, storing trial materials, intervention delivery timing and adherence to intervention protocols. The delivering physiotherapist received feedback at the end of each quality assurance visit. If protocol adherence was below the required standard, further measures (e.g. retraining) were to be instituted after discussion with the trial team and site. Common problems or failings were also shared across sites through regular trial newsletters, e-mails and meetings.

Clinicians delivering the GRASP trial intervention were asked to complete treatment logs at the end of each session. The logs included a checklist that documented the key elements of the session (e.g. exercises prescribed, sets and repetitions, load, injection type and dosage/volume). These were returned to the central GRASP trial team, which reviewed the data for each participant and entered them onto the trial database. For the progressive-exercise intervention, exercise prescription details were checked on arrival. An assessment of progression of exercises over the sessions was not part of the ongoing monitoring. As described above, participants were also allowed to maintain or regress their exercises over the sessions, if clinically appropriate. However, these data were carefully recorded, analysed on completion of the trial and are reported in Chapter 4.

Custom-designed reports of treatment log data were generated and regularly reviewed during the conduct of the trial for central monitoring of the intervention delivery, including the timing, number and duration of sessions, injection details (e.g. volume/dosage), content of treatment sessions, participant attendance and paperwork return. Intervention delivery was, therefore, monitored 
throughout the trial on a site-by-site and overall basis. Ongoing monitoring during the trial aimed to reveal deviations or discrepancies from the trial protocol so that they could be investigated and, if required, measures taken to address them at an early stage throughout the trial.

Completion of the best-practice advice intervention was defined as attendance at one session. For the progressive-exercise intervention, completion of treatment was defined as attendance at all six treatment sessions, discharged by the treating physiotherapist or referred on for further investigation/treatment (e.g. to orthopaedic consultant). Partial completion was defined as attendance of at least one session but not fulfilling the criteria for full completion described above.

\section{Conclusion}

This chapter described the development and details of the GRASP trial interventions for treating rotator cuff disorders tested as part of the trial. The results on how the interventions were implemented and the outcomes from onsite monitoring are detailed in Chapter 4. 



\section{Chapter 4 Results}

eproduced with permission from Hopewell et al.82 @ 2021 The Author(s). Published by Elsevier Ltd. This is an Open Access article under the CC BY 4.0 license.

\section{Trial sites}

\section{Centre characteristics}

Twenty English NHS trusts participated in the GRASP trial (see Appendix 4, Table 28). The recruitment period (20 February 2017 to 2 May 2019) at each trust varied from 9 to 26 months, with resulting variation in the number of participants recruited at each site.

\section{Participating clinicians}

A total of 223 clinicians across the 20 trial sites were involved in providing the study treatment interventions. Fifty-three physiotherapists and three doctors delivered subacromial corticosteroid injections to $329(97 \%)$ and 10 (3\%) participants, respectively. The best-practice advice and progressiveexercise interventions were delivered by 83 and 104 physiotherapists to 324 and 339 participants, respectively (see Appendix 4, Table 29). The physiotherapists and doctors worked in hospital physiotherapy, orthopaedic outpatient departments or at satellite clinics (e.g. general practices). Nine physiotherapists at two sites were working for a private company contracted to provide services to NHS patients [Buckinghamshire Musculoskeletal Integrated Care Service (High Wycombe, UK) and Medway Community Healthcare (Rochester, UK)]. The number of physiotherapists involved at each site varied from 3 to 43 (see Appendix 4, Table 28). Several physiotherapists provided both the corticosteroid injection and either the best-practice advice or progressive-exercise intervention. Two physiotherapists swapped treatment groups during the trial because of staffing issues at sites and, therefore, delivered both exercise interventions.

The level of experience of each physiotherapist (as determined by Agenda for Change pay banding)72 is described in Appendix 4, Table 29. Sixty-four per cent (34/53) of physiotherapists delivering injections were band 8 . Exercise interventions were predominantly delivered by band 6 and 7 physiotherapists ( $73 \%$ and $80 \%$ for best-practice advice and progressive-exercise interventions, respectively).

\section{Participant flow}

The overall flow of participants through the study is described in the CONSORT (Consolidated Standards of Reporting Trials) diagram (Figure 3). Further details for each stage are provided in the following sections.

\section{Recruitment}

\section{Screening}

Screening and recruitment took place between February 2017 and April 2019. A total of 2287 patients were screened and assessed to see whether or not they met the GRASP trial eligibility criteria (Table 5). A total of 1284 (56\%) patients were eligible and 708 agreed to participate. Of those participants who did not meet the eligibility criteria (1003/2287), the most common reasons were owing to another shoulder disorder (e.g. inflammatory arthritis, frozen shoulder, glenohumeral joint instability) or red flags consistent with BESS criteria ( $n=434,43 \%$ ), or had received corticosteroid injection or physiotherapy for shoulder pain in last 6 months $(n=167,17 \%)$. Of those participants who were eligible but declined to participate $(n=576)$, the most common reasons given were not interested in taking part in research 


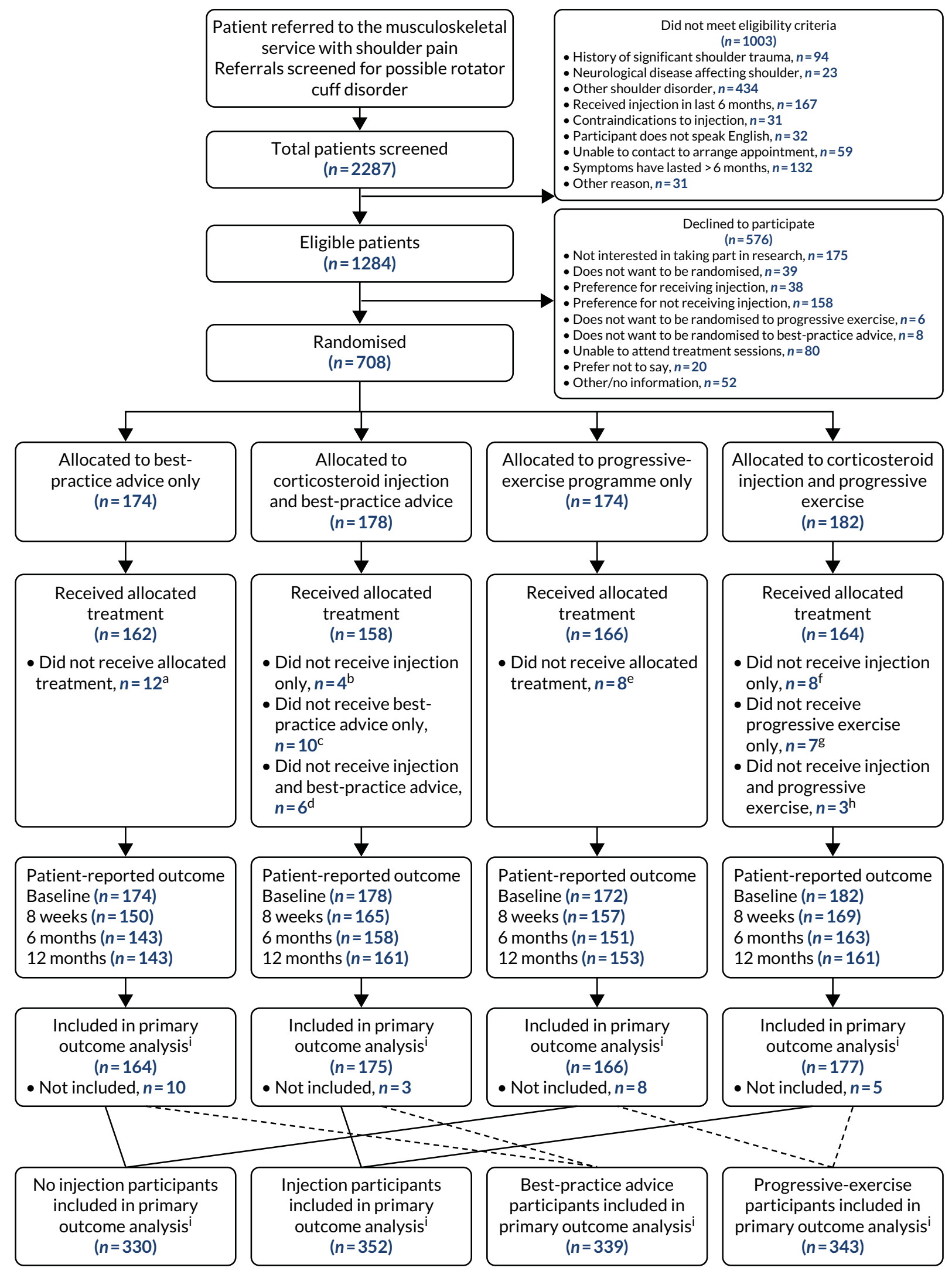

FIGURE 3 Participant flow diagram. a, Reasons for not receiving best-practice advice: participant did not attend session $(n=5)$, withdrawal $(n=4)$ and other medical condition $(n=3)$; b, reasons for not receiving injection only: participant declined treatment $(n=2)$ and contraindications (taking anticoagulants, $n=1$; previous reaction to injection, $n=1$ ); 
c, reasons for not receiving best-practice advice only: participant did not attend session $(n=5)$, other medical condition $(n=2)$ and received progressive exercise in error $(n=3)$; d, reasons for not receiving injection and best-practice advice: participant did not attend session $(n=1)$, participant declined treatment $(n=2)$, other medical condition $(n=2)$ and previous reaction to injection and received non-GRASP trial treatment $(n=1)$; e, reason for not receiving progressive exercise: participant did not attend session $(n=3)$, received best-practice advice in error $(n=2)$, received injection in error $(n=1)$, received non-GRASP trial treatment $(n=1)$ and withdrawal $(n=1)$; f, reasons for not receiving injection only: participant declined treatment $(n=5)$ and clinician declined treatment $(n=3) ; g$, reasons for not receiving progressive exercise only: received best-practice advice in error $(n=2)$, received non-GRASP trial treatment $(n=3)$, other medical condition $(n=1)$ and participant did not attend session $(n=1) ; h$, reasons for not receiving injection and progressive exercise: participant did not attend session $(n=2)$ and other medical condition $(n=1)$; and i, included in the analysis' is all participants with at least one follow-up time point SPADI outcome and the baseline variables used in the model.

TABLE 5 Number of participants screened by site

\begin{tabular}{|c|c|c|c|c|}
\hline Site & $\begin{array}{l}\text { Screened } \\
(N=2287), n\end{array}$ & $\begin{array}{l}\text { Ineligible } \\
(N=1003), n\end{array}$ & $\begin{array}{l}\text { Declined } \\
(N=576), n\end{array}$ & $\begin{array}{l}\text { Randomised } \\
(N=708), n\end{array}$ \\
\hline $\begin{array}{l}\text { University Hospitals of Derby and Burton NHS } \\
\text { Foundation Trust }\end{array}$ & 152 & 55 & 51 & 46 \\
\hline East Lancashire Hospitals NHS Trust & 387 & 193 & 92 & 102 \\
\hline Gloucestershire Hospitals NHS Foundation Trust & 435 & 181 & 134 & 120 \\
\hline Birmingham Community Healthcare NHS Trust & 81 & 17 & 35 & 29 \\
\hline Sandwell and West Birmingham Hospitals NHS Trust & 89 & 23 & 31 & 35 \\
\hline Buckinghamshire Musculoskeletal Integrated Care Service & 41 & 22 & 5 & 14 \\
\hline East Cheshire NHS Trust & 44 & 17 & 13 & 14 \\
\hline Bedfordshire Hospitals NHS Foundation Trust & 72 & 39 & 10 & 23 \\
\hline Wirral University Teaching Hospital NHS Foundation Trust & 58 & 30 & 15 & 13 \\
\hline Medway Community Healthcare & 34 & 3 & & 31 \\
\hline Bristol Community Health & 6 & 1 & 1 & 4 \\
\hline Somerset Partnership NHS Foundation Trust & 255 & 167 & 39 & 49 \\
\hline $\begin{array}{l}\text { Doncaster and Bassetlaw Teaching Hospitals NHS } \\
\text { Foundation Trust }\end{array}$ & 81 & 28 & 20 & 33 \\
\hline Sherwood Forest Hospitals NHS Foundation Trust & 66 & 14 & 23 & 29 \\
\hline Kent Community Health NHS Foundation Trust & 62 & 10 & 16 & 36 \\
\hline Northern Devon Healthcare NHS Trust & 127 & 50 & 41 & 36 \\
\hline Airedale NHS Foundation Trust & 69 & 36 & 8 & 25 \\
\hline North West Boroughs Healthcare NHS Foundation Trust & 163 & 92 & 31 & 40 \\
\hline Warrington and Halton Hospitals NHS Foundation Trust & 52 & 25 & 9 & 18 \\
\hline Staffordshire and Stoke-on-Trent Partnership NHS Trust & 13 & & 2 & 11 \\
\hline
\end{tabular}


( $n=175,30 \%$ ) or had a treatment preference for not receiving injection ( $n=158,27 \%)$. For full details see Appendix 4, Table 30. The mean age of those who declined to participate was 55 years (median 56 years, IQR 46-65 years) and 53\% $(n=306)$ were female.

A total of 708 participants were recruited between March 2017 and May 2019 (see Appendix 4, Figure 24). This exceeded the target of 704 participant; four participants were randomised once the target was reached as they were already in the process of joining the study.

\section{Baseline data}

\section{Baseline characteristics of participants}

The baseline characteristics of participants recruited into the trial are shown in Table 6 (for more detail see Appendix 4, Tables 31-33). Intervention groups were generally well matched in terms of baseline demographic data and primary and secondary outcome measurements. Stratification factors used in the randomisation are shown in Appendix 4, Table 34. The majority of participants were white British (89.6\%), with similar numbers of men and women (50.7\% vs. 49.3\%). The overall mean age was 55.5 (SD 13.1) years. The majority of participants reported being married (64.8\%) or living with a partner (13.1\%). More than two-thirds of participants were classified as either overweight (38.7\%) or obese (30.6\%). Approximately half (58.9\%) reported being in employment, with one-quarter (25.8\%) being retired. The majority of participants reported being right-handed (87\%) and the numbers were similar in terms of whether the right or left shoulder was affected (49.3\% vs. 46.9\%). The average duration of rotator cuff symptoms was 4 (median 4, IQR 3-6) months.

TABLE 6 Participant baseline characteristics ('inside the table')

\begin{tabular}{|c|c|c|c|c|}
\hline Characteristic & $\begin{array}{l}\text { Best-practice } \\
\text { advice } \\
(\mathrm{N}=174)\end{array}$ & $\begin{array}{l}\text { Injection plus } \\
\text { best-practice } \\
\text { advice } \\
(\mathrm{N}=178)\end{array}$ & $\begin{array}{l}\text { Progressive } \\
\text { exercise } \\
(N=174)\end{array}$ & $\begin{array}{l}\text { Injection plus } \\
\text { progressive } \\
\text { exercise } \\
(\mathbf{N}=182)\end{array}$ \\
\hline Age (years), mean (SD), $n$ & 55.9 (13.1), 174 & 56.5 (12.4), 178 & 54.6 (13.7), 174 & 54.8 (13.2), 182 \\
\hline $18-35$ years, $n(\%)^{\mathrm{a}}$ & $11(6.3)$ & $13(7.3)$ & $14(8.0)$ & $17(9.3)$ \\
\hline$\geq 36$ years, $n(\%)^{a}$ & $163(93.7)$ & $165(92.7)$ & $160(92.0)$ & $165(90.7)$ \\
\hline \multicolumn{5}{|l|}{ Sex, $n(\%)^{a}$} \\
\hline Male & $87(50.0)$ & $89(50.0)$ & $90(51.7)$ & $93(51.1)$ \\
\hline Female & $87(50.0)$ & $89(50.0)$ & $84(48.3)$ & $89(48.9)$ \\
\hline \multicolumn{5}{|l|}{ Ethnicity, n (\%) } \\
\hline White & $160(92.0)$ & $167(93.8)$ & $154(88.5)$ & $167(91.8)$ \\
\hline Other & $14(8.0)$ & $11(6.2)$ & $18(10.4)$ & $15(8.2)$ \\
\hline Missing & $0(0.0)$ & $0(0.0)$ & $2(1.1)$ & $0(0.0)$ \\
\hline \multicolumn{5}{|l|}{ Marital status, $n(\%)$} \\
\hline Married/civil union & 118 (67.8) & $107(60.1)$ & $114(65.5)$ & $120(65.9)$ \\
\hline Living with partner & $24(13.8)$ & $23(12.9)$ & $22(12.6)$ & $24(13.2)$ \\
\hline Other & $32(18.4)$ & $48(27.0)$ & $36(20.8)$ & $38(20.9)$ \\
\hline Missing & $0(0.0)$ & $0(0.0)$ & $2(1.1)$ & $0(0.0)$ \\
\hline Height (m), mean (SD), $n$ & 1.7 (0.0), 174 & $1.7(0.0), 178$ & 1.7 (0.2), 172 & $1.7(0.2), 182$ \\
\hline Weight (kg), mean (SD), $n$ & 80.9 (16.6), 174 & 82.9 (17.4), 176 & 81.2 (18.4), 170 & 81.7 (18.0), 180 \\
\hline
\end{tabular}


TABLE 6 Participant baseline characteristics ('inside the table') (continued)

\begin{tabular}{|c|c|c|c|c|}
\hline Characteristic & $\begin{array}{l}\text { Best-practice } \\
\text { advice } \\
(\mathrm{N}=174)\end{array}$ & $\begin{array}{l}\text { Injection plus } \\
\text { best-practice } \\
\text { advice } \\
(\mathrm{N}=178)\end{array}$ & $\begin{array}{l}\text { Progressive } \\
\text { exercise } \\
(N=174)\end{array}$ & $\begin{array}{l}\text { Injection plus } \\
\text { progressive } \\
\text { exercise } \\
(\mathrm{N}=182)\end{array}$ \\
\hline Body mass index $\left(\mathrm{kg} / \mathrm{m}^{2}\right)$, mean (SD), $n$ & 27.9 (5.0), 174 & $28.7(5.4), 176$ & $28.0(5.4), 170$ & $28.1(4.8), 180$ \\
\hline Underweight $\left(<18.5 \mathrm{~kg} / \mathrm{m}^{2}\right), n(\%)$ & $3(1.7)$ & $1(0.6)$ & $0(0.0)$ & $0(0.0)$ \\
\hline Normal weight (18.5-24.9 kg/m²), n (\%) & $51(29.3)$ & $51(28.7)$ & $50(28.7)$ & $53(29.1)$ \\
\hline Overweight (25-29.9 kg/m²), n (\%) & $68(39.1)$ & $63(35.4)$ & $73(42.0)$ & $70(38.5)$ \\
\hline Obese $\left(\geq 30 \mathrm{~kg} / \mathrm{m}^{2}\right), n(\%)$ & $52(29.9)$ & $61(34.3)$ & $47(27.0)$ & $57(31.3)$ \\
\hline Missing & $0(0.0)$ & $2(1.1)$ & $4(2.3)$ & $2(1.1)$ \\
\hline \multicolumn{5}{|l|}{ Smoking status, $n$ (\%) } \\
\hline Never smoked & $85(48.9)$ & $100(56.2)$ & $99(56.9)$ & $101(55.5)$ \\
\hline Former smoker & $66(37.9)$ & $66(37.1)$ & $61(35.1)$ & $63(34.6)$ \\
\hline Current smoker & $23(13.2)$ & $12(6.7)$ & $12(6.9)$ & $18(9.9)$ \\
\hline Missing & $0(0.0)$ & $0(0.0)$ & $2(1.1)$ & $0(0.0)$ \\
\hline $\begin{array}{l}\text { Cigarettes smoked per day } \\
\text { (current smoker), median (IQR), } n\end{array}$ & $10(10,15), 21$ & $15(5,18), 11$ & 13 (8, 18), 12 & $10(6,12), 17$ \\
\hline $\begin{array}{l}\text { Cigarettes smoked per day } \\
\text { (former smoker), median (IQR), } n\end{array}$ & $13.5(6-20), 66$ & $15.0(10-20), 66$ & 10.0 (5-20), 60 & 10.0 (8-20), 63 \\
\hline Symptoms duration, median (IQR), $n$ & $4.0(2-6), 173$ & $4.0(3-6), 178$ & $4.0(3-6), 172$ & $4.0(3-6), 182$ \\
\hline \multicolumn{5}{|l|}{ Affected shoulder, $n$ (\%) } \\
\hline Left shoulder & 89 (51.1) & $78(43.8)$ & $83(47.7)$ & $82(45.1)$ \\
\hline Right shoulder & $78(44.8)$ & $94(52.8)$ & $84(48.3)$ & $93(51.1)$ \\
\hline Both shoulders & $7(4.0)$ & $6(3.4)$ & $5(2.9)$ & $7(3.8)$ \\
\hline Missing & $0(0.0)$ & $0(0.0)$ & $2(1.1)$ & $0(0.0)$ \\
\hline \multicolumn{5}{|l|}{ Hand dominance, $n$ (\%) } \\
\hline Left-handed & $13(7.5)$ & $16(9.0)$ & $21(12.1)$ & $21(11.5)$ \\
\hline Right-handed & $157(90.2)$ & $153(86.0)$ & $148(85.1)$ & $158(86.8)$ \\
\hline Both & $4(2.3)$ & $9(5.1)$ & $3(1.7)$ & $3(1.6)$ \\
\hline Missing & $0(0.0)$ & $0(0.0)$ & $2(1.1)$ & $0(0.0)$ \\
\hline \multicolumn{5}{|l|}{ Current work status, $n$ (\%) } \\
\hline Retired & $44(25.3)$ & $50(28.1)$ & $40(23.0)$ & $49(26.9)$ \\
\hline Semi-retired & $13(7.5)$ & $10(5.6)$ & $9(5.2)$ & $7(3.8)$ \\
\hline Employed & $84(48.2)$ & 91 (51.1) & $98(56.3)$ & $82(45.1)$ \\
\hline Other & $33(19.0)$ & $27(15.2)$ & $25(14.4)$ & $43(23.7)$ \\
\hline Missing & $0(0.0)$ & $0(0.0)$ & $2(1.1)$ & $1(0.5)$ \\
\hline \multicolumn{5}{|l|}{ SPADI, mean (SD), $n$} \\
\hline Pain subscale & 66.0 (17.7), 174 & 64.2 (18.3), 178 & 60.7 (17.1), 172 & 64.6 (17.5), 182 \\
\hline Function subscale & 48.1 (23.2), 174 & 46.3 (22.0), 178 & 40.3 (21.0), 172 & 42.6 (21.6), 182 \\
\hline SPADI overall & 57.0 (19.2), 174 & 55.3 (18.9), 178 & 50.5 (17.5), 172 & 53.6 (17.8), 182 \\
\hline
\end{tabular}


TABLE 6 Participant baseline characteristics ('inside the table') (continued)

\begin{tabular}{|c|c|c|c|c|}
\hline Characteristic & $\begin{array}{l}\text { Best-practice } \\
\text { advice } \\
(\mathrm{N}=174)\end{array}$ & $\begin{array}{l}\text { Injection plus } \\
\text { best-practice } \\
\text { advice } \\
(\mathbf{N}=178)\end{array}$ & $\begin{array}{l}\text { Progressive } \\
\text { exercise } \\
(N=174)\end{array}$ & $\begin{array}{l}\text { Injection plus } \\
\text { progressive } \\
\text { exercise } \\
(\mathbf{N}=182)\end{array}$ \\
\hline FABQ-PA, mean (SD), $n$ & 15.6 (5.8), 172 & $15.7(5.4), 177$ & $14.2(5.5), 172$ & 14.8 (5.3), 182 \\
\hline PSEQ-2, mean (SD), $n$ & 9.6 (2.4), 174 & 9.5 (2.3), 178 & 9.8 (2.3), 172 & 9.7 (2.3), 182 \\
\hline ISI, mean (SD), $n$ & 11.0 (6.7), 173 & $10.4(6.2), 176$ & 9.5 (5.7), 170 & 11.1 (6.4), 180 \\
\hline RDA, mean (SD), $n$ & 8.0 (2.7), 174 & 8.2 (2.5), 178 & $7.6(2.7), 172$ & 7.7 (2.6), 182 \\
\hline \multicolumn{5}{|l|}{ Trial centre, $n(\%)^{a}$} \\
\hline 1 & $12(6.9)$ & $9(5.1)$ & $12(6.9)$ & $13(7.1)$ \\
\hline 2 & $26(14.9)$ & $26(14.6)$ & $25(14.4)$ & $25(13.7)$ \\
\hline 3 & $29(16.7)$ & $30(16.9)$ & $31(17.8)$ & $30(16.5)$ \\
\hline 4 & $8(4.6)$ & 7 (3.9) & $7(4.0)$ & $7(3.8)$ \\
\hline 5 & $9(5.2)$ & $8(4.5)$ & $8(4.6)$ & $10(5.5)$ \\
\hline 6 & $3(1.7)$ & $3(1.7)$ & $4(2.3)$ & $4(2.2)$ \\
\hline 7 & $3(1.7)$ & $4(2.2)$ & $3(1.7)$ & $4(2.2)$ \\
\hline 8 & $5(2.9)$ & $6(3.4)$ & $7(4.0)$ & $5(2.7)$ \\
\hline 9 & $4(2.3)$ & $3(1.7)$ & $4(2.3)$ & $2(1.1)$ \\
\hline 10 & $7(4.0)$ & 7 (3.9) & $8(4.6)$ & $9(4.9)$ \\
\hline 11 & $1(0.6)$ & $1(0.6)$ & $1(0.6)$ & $1(0.5)$ \\
\hline 12 & $12(6.9)$ & $12(6.7)$ & $13(7.5)$ & $12(6.6)$ \\
\hline 13 & $8(4.6)$ & $8(4.5)$ & $7(4.0)$ & $10(5.5)$ \\
\hline 14 & $7(4.0)$ & 9 (5.1) & $6(3.4)$ & 7 (3.8) \\
\hline 15 & $9(5.2)$ & $9(5.1)$ & $8(4.6)$ & $10(5.5)$ \\
\hline 16 & $9(5.2)$ & $10(5.6)$ & $8(4.6)$ & $9(4.9)$ \\
\hline 17 & $6(3.4)$ & 7 (3.9) & $6(3.4)$ & $6(3.3)$ \\
\hline 18 & $9(5.2)$ & 11 (6.2) & $9(5.2)$ & $11(6.0)$ \\
\hline 19 & $2(1.1)$ & $3(1.7)$ & $3(1.7)$ & $3(1.6)$ \\
\hline 20 & $5(2.9)$ & $5(2.8)$ & $4(2.3)$ & $4(2.2)$ \\
\hline
\end{tabular}

a Stratification factor used in randomisation.

The overall mean baseline SPADI score was 54.1 (SD 18.5) (on a scale of 0-100, with higher values being worse), suggesting a moderate level of disability, with higher levels of shoulder pain (mean SPADI pain subscale 63.9, SD 17.1) compared with impairment of shoulder function (mean SPADI function subscale 44.3, SD 22.1). Overall baseline scores for secondary outcome measures were mean 15.1 (SD 5.5) for FABQ-PA, mean 9.7 (SD 2.3) for PSEQ-2, mean 10.5 (SD 6.3) for ISI and mean 7.9 (SD 2.6) for RDA. Mean baseline values were in the moderate to low range, suggesting that a participant's rotator cuff disorder may not be having considerable impact on sleep and daily activities despite higher levels of pain. 


\section{Follow-up}

Participants were followed up at 8 weeks and at 6 and 12 months; overall response rates were $91 \%$ (641/708), 87\% (615/708) and 87\% (618/708), respectively. Response rates were generally well balanced across intervention groups. Appendix 4, Table 35, shows the number of responses according to type of follow-up (i.e. postal or telephone questionnaire).

\section{Withdrawals}

Overall, 26 (3.7\%) participants withdrew from the trial: 15 withdrew from the intervention delivery only and 11 withdrew from both the intervention and follow-up questionnaire completion. Numbers were very similar across intervention groups. One participant withdrew completely from the trial and withdrew permission to use their data. Details of the reasons for withdrawal by intervention group are shown in Table 7. Two participants died and both deaths were unrelated to the trial intervention(s).

\section{Intervention delivery}

\section{Compliance with intervention}

Of the 708 participants randomised, the majority received treatment as allocated, with 94\% (339/360) of participants randomised to receive subacromial corticosteroid injection prior to either the bestpractice advice or progressive-exercise intervention receiving the injection as allocated (Table 8).

TABLE 7 Participant withdrawals and reason for withdrawal

\begin{tabular}{|c|c|c|c|c|c|c|}
\hline & $\begin{array}{l}\text { Best- } \\
\text { practice } \\
\text { advice } \\
(\mathrm{N}=174)\end{array}$ & $\begin{array}{l}\text { Injection } \\
\text { plus best- } \\
\text { practice } \\
\text { advice } \\
(\mathbf{N}=178)\end{array}$ & $\begin{array}{l}\text { Progressive } \\
\text { exercise } \\
(\mathrm{N}=174)\end{array}$ & & $\begin{array}{l}\text { on } \\
\text { ssive } \\
\text { se } \\
32 \text { ) }\end{array}$ & $\begin{array}{l}\text { Overall } \\
(N=708)\end{array}$ \\
\hline Reason for withdrawal & $\%$ & $\%$ & $\%$ & $n$ & $\%$ & $\%$ \\
\hline
\end{tabular}

Overall $^{\mathrm{a}}$

Complete withdrawal from use of data

Withdrawn from intervention ${ }^{\mathrm{a}}$

Believes intervention not working

Change of diagnosis since randomisation

Had treatment preference

No reason given

Pain has improved

Unable to continue with treatment

Withdrawn from intervention and future assessments $^{\mathrm{a}}$

Believes intervention not working

Change of diagnosis since randomisation

No reason given

Missing

$\begin{array}{rrrrrrrrrr}0 & 0 & 0 & 0 & 1 & 1 & 0 & 0 & 1 & 0 \\ 3 & 2 & 4 & 2 & 2 & 1 & 6 & 3 & 15 & 2 \\ 1 & 1 & 1 & 1 & 0 & 0 & 0 & 0 & 2 & 0 \\ 0 & 0 & 3 & 2 & 1 & 1 & 4 & 2 & 8 & 1 \\ 1 & 1 & 0 & 0 & 0 & 0 & 0 & 0 & 1 & 0 \\ 1 & 1 & 0 & 0 & 0 & 0 & 0 & 0 & 1 & 0 \\ 0 & 0 & 0 & 0 & 1 & 1 & 0 & 0 & 1 & 0 \\ 0 & 0 & 0 & 0 & 0 & 0 & 2 & 1 & 2 & 0 \\ 4 & 2 & 3 & 2 & 3 & 2 & 0 & 0 & 11 & 2 \\ 0 & 0 & 1 & 1 & 1 & 1 & 0 & 0 & 2 & 0 \\ 3 & 2 & 0 & 0 & 1 & 1 & 0 & 0 & 4 & 1 \\ 1 & 1 & 2 & 1 & 1 & 1 & 0 & 0 & 4 & 1 \\ 0 & 0 & 0 & 0 & 0 & 0 & 0 & 0 & 1 & 0\end{array}$

a Death (unrelated to the study intervention) was reported for two participants at the 6-month follow-up. One participant withdrew from the intervention and another was classified as withdrawn from intervention and future assessments following notice of their death. 
TABLE 8 Intervention received

\begin{tabular}{|c|c|c|c|c|}
\hline Intervention received & $\begin{array}{l}\text { Best- } \\
\text { practice } \\
\text { advice }^{\mathrm{a}} \\
(\mathrm{N}=174)\end{array}$ & $\begin{array}{l}\text { Injection plus } \\
\text { best-practice } \\
\text { advice }^{\mathrm{a}}(\mathrm{N}=178)\end{array}$ & $\begin{array}{l}\text { Progressive } \\
\text { exercise }^{b} \\
(N=174)\end{array}$ & $\begin{array}{l}\text { Injection plus } \\
\text { progressive } \\
\text { exercise }^{\mathbf{b}} \\
\text { (N= 182) }\end{array}$ \\
\hline Injection received, $n$ (\%) & & $168(94)$ & & $171(94)$ \\
\hline Injection not received, $n$ (\%) & & $10(6)$ & & $11(6)$ \\
\hline Did not attend, $n$ (participants) & & 1 & & 2 \\
\hline Participant declined, $n$ (participants) & & 4 & & 4 \\
\hline Clinician declined, $n$ (participants) & & 5 & & 5 \\
\hline Received extra injection, $n$ (participants) & & 0 & & 2 \\
\hline Completed exercise treatment, ${ }^{c} n(\%)$ & $162(93)$ & $162(91)$ & $138(79)$ & $139(76)$ \\
\hline Partial exercise completion, ${ }^{d} n(\%)$ & & & $29(17)$ & $33(18)$ \\
\hline Received no treatment, $n(\%)$ & $12(7)$ & $16(9)$ & $7(4)$ & $10(5)$ \\
\hline $\begin{array}{l}\text { Did not attend/unable to contact, } \\
n \text { (participants) }\end{array}$ & 5 & 6 & 3 & 3 \\
\hline Withdrawal/declined, $n$ (participants) & 4 & 2 & 1 & 0 \\
\hline Other condition, $n$ (participants) & 3 & 4 & 0 & 2 \\
\hline $\begin{array}{l}\text { Received wrong trial intervention, } \\
n \text { (participants) }\end{array}$ & 0 & 3 & 2 & 2 \\
\hline $\begin{array}{l}\text { Received non-GRASP trial treatment, } \\
n \text { (participants) }\end{array}$ & 0 & 1 & 1 & 3 \\
\hline Median number of sessions (IQR) & $1(1-1)$ & $1(1-1)$ & $4(3-6)$ & $4(3-5)$ \\
\hline \multicolumn{5}{|l|}{ Attendance, $n(\%)$} \\
\hline Session 1 & $162(93)$ & $162(91)$ & $167(96)$ & $172(95)$ \\
\hline Session 2 & & & $161(93)$ & $160(88)$ \\
\hline Session 3 & & & $144(83)$ & $136(75)$ \\
\hline Session 4 & & & $101(58)$ & $100(55)$ \\
\hline Session 5 & & & $72(41)$ & $69(38)$ \\
\hline Session 6 & & & $44(25)$ & $38(21)$ \\
\hline Participants receiving additional sessions, $n$ (\%) & $3(2)$ & $5(3)$ & $3(2)$ & $2(1)$ \\
\hline Additional contact sessions, ${ }^{\mathrm{e}} n$ & 4 & 6 & 3 & 5 \\
\hline Telephone & 2 & 1 & 0 & 1 \\
\hline Face to face & 2 & 5 & 3 & 4 \\
\hline
\end{tabular}

a Maximum of one session of best-practice advice.

b Up to six sessions of progressive exercise.

c Best-practice advice: attendance at one or more sessions. Progressive exercise: six sessions attended, discharged by clinician as treatment completed (as marked on treatment log), discharged by clinician following patient-initiated follow-up period with no further contact or referred on for further investigation/treatment.

d Defined as attendance at one or more sessions.

e Some participants received more than one additional contact. 
For the exercise interventions, there was no difference in attendance rates between those receiving the exercise interventions only and those who received exercise in conjunction with a corticosteroid injection. Overall, $92 \%$ (324/352) of participants of those allocated to receive the best-practice advice intervention attended, and 95\% (339/356) of participants allocated to receive progressive exercise either partially or fully completed the exercise intervention.

The proportion of participants not attending any treatment sessions was roughly equal between intervention groups. For the progressive-exercise intervention, just over half of participants received four out of a maximum of six sessions, with almost $80 \%$ receiving at least three sessions. Note that, participants could be discharged prior to attending six sessions if their symptoms had improved sufficiently.

A small number of participants received extra treatment over and above the interventions defined by the GRASP trial protocol. Two sites reported that two participants received a second subacromial corticosteroid injection in addition to that received as part of their treatment allocation. With regard to the exercise interventions, eight participants allocated to receive best-practice advice received a total of 10 additional sessions. For progressive exercise, five participants received a total of eight additional sessions over and above the six-session maximum. Three of the 13 participants who received additional sessions required further reassurance regarding their condition and/or the exercise programme, four required further session(s) before treatment was concluded satisfactorily, three reported a re-occurrence or suffered re-injury and three reported no improvement in symptoms. There was no sizeable difference in the proportion of participants receiving additional sessions (nor the reasons for further contact) between the four intervention groups.

\section{Timing of delivery of interventions}

Appendix 4, Table 36, presents median time from randomisation to first appointments for each intervention group. Subacromial corticosteroid injections prior to receiving the best-practice advice or progressiveexercise interventions were largely delivered within 10 days of randomisation in accordance with the GRASP trial protocol. The first session of the exercise interventions was also delivered within the required time frames for all trial groups, with the majority of participants being seen between 14 and 28 days after randomisation. Participants randomised to injection received their first exercise session 6 days later, on average, than those who were not allocated to receive an injection, possibly because of logistical issues with booking appointments. The progressive-exercise intervention was generally completed within the recommended 16-week time frame, with the median time corresponding to between 13 and 15 weeks for both intervention groups. The initial delay experienced by those who received an injection prior to their exercise sessions is reflected in the longer time to the last session, although the 'actual' exercise treatment period (i.e. first to last exercise session) was almost identical for both progressive-exercise arms.

\section{Content and adherence to interventions}

Injecting clinicians and physiotherapists completed a treatment log for each session, detailing the type of treatment provided.

\section{Subacromial corticosteroid injection}

Triamcinolone acetonide was the most commonly delivered corticosteroid, accounting for $64 \%(218 / 339)$ of injections delivered overall. The remaining 36\% (121/339) of participants received methylprednisolone injection (Table 9). Lidocaine $1 \%$ was the most commonly delivered local anaesthetic $(294 / 339,87 \%)$, with only $10 \%$ (33/339) receiving $0.5 \%$ bupivacaine hydrochloride. Twelve participants received no local anaesthetic (4\%), usually because of clinician preference or previous history of adverse reactions. The majority of injections were administered into the posterior subacromial space $(278 / 339,82 \%)$. There were no meaningful differences in injection delivery between the two injection groups. The median dose of corticosteroid prescribed was $40 \mathrm{mg}$ (range of 10-40 mg for methylprednisolone and $20-40 \mathrm{mg}$ for triamcinolone acetonide) and the median volume of local anaesthetic was 4 (range of 0.2-5) $\mathrm{ml}$ for $1 \%$ lidocaine and 9 (range $2-10$ ) $\mathrm{ml}$ for $0.5 \%$ bupivacaine hydrochloride, indicating that the injection protocol was adhered to by all injecting therapists. 
TABLE 9 Content of subacromial corticosteroid injection

\begin{tabular}{|c|c|c|c|}
\hline Content of injection ${ }^{a}$ & $\begin{array}{l}\text { Injection plus progressive } \\
\text { exercise ( } \mathrm{N} \text { received } \\
\text { injection }=171 \text { ) }\end{array}$ & $\begin{array}{l}\text { Injection plus } \\
\text { best-practice } \\
\text { advice ( } N \text { received } \\
\text { injection = 168) }\end{array}$ & $\begin{array}{l}\text { Total (N received } \\
\text { injection }=339 \text { ) }\end{array}$ \\
\hline \multicolumn{4}{|c|}{ Corticosteroid, $n$ (\%), median dose/volume (IQR) } \\
\hline Methylprednisolone (mg) & 60 (35), 40 (32-40) & $61(36), 40(30-30)$ & $121(36), 40(30-40)$ \\
\hline Triamcinolone acetonide (mg) & 111 (65), 40 (20-20) & $107(64), 40(20-40)$ & $218(64), 40(20-40)$ \\
\hline \multicolumn{4}{|c|}{ Local anaesthetic, $n(\%)$, median dose/volume (IQR) } \\
\hline $1 \%$ lidocaine (ml) & $148(87), 4(2-5)$ & $146(87), 4(2-5)$ & $294(87), 4(2-5)$ \\
\hline $0.5 \%$ bupivacaine hydrochloride $(\mathrm{ml})$ & 18 (11), 9 (9-9) & 15 (9), 9 (9-10) & 33 (10), 9 (9-9) \\
\hline None & $5(3)$ & $7(4)$ & $12(4)$ \\
\hline \multicolumn{4}{|l|}{ Shoulder, $n$ (\%) } \\
\hline Right shoulder & $91(53)$ & $90(54)$ & $181(53)$ \\
\hline Left shoulder & $80(47)$ & $78(46)$ & $158(47)$ \\
\hline \multicolumn{4}{|l|}{ Injection site, $n$ (\%) } \\
\hline Anterior subacromial & $13(7)$ & $14(8)$ & $27(8)$ \\
\hline Posterior subacromial & $142(83)$ & $136(81)$ & $278(82)$ \\
\hline Lateral subacromial & $15(9)$ & $15(9)$ & $30(9)$ \\
\hline Glenohumeral joint & $1(1)$ & $3(2)$ & $4(1)$ \\
\hline
\end{tabular}

\section{Best-practice advice intervention}

Table 10 provides a summary of the treatment logs per session for the best-practice advice intervention. The figures indicate a high level of compliance (as recorded by the treating physiotherapist) with the treatment protocols with regard to provision of exercises, advice, information and treatment compliance strategies. Resisted (isotonic) shoulder external rotation in neutral and active-assisted (wall) shoulder flexion were the most commonly prescribed starting exercises. Ninety-eight per cent (318/324) of participants were prescribed one or two exercises at their treatment session. There were no meaningful differences between those randomised to receive corticosteroid injection plus band and those who received best-practice advice only.

\section{Progressive-exercise intervention}

Table 11 (see also Appendix 4, Table 37) provides details of the delivery of the progressive-exercise intervention for each session, as recorded by the treating physiotherapist on the participant treatment log. Similar to the best-practice advice intervention, the figures illustrate a high level of compliance in delivering the various components of the intervention. The most commonly prescribed exercises were from the core exercise section, aimed at strengthening the posterior rotator cuff muscles. All participants in the progressive-exercise intervention received the required number of exercises (i.e. three or less). Level 2 strengthening exercises were the core element of the progressive-exercise intervention and, when these were prescribed, participants were initially expected to perform one set of up to eight repetitions at a load of 3-4 on the modified 10-point Borg scale. Of the 339 participants who attended, 98\% (332/339) received these core exercises. In accordance with the trial protocol, data from the treatment logs suggest that on initial presentation, $72 \%(238 / 332)$ of participants 
TABLE 10 Content of best-practice advice intervention

\begin{tabular}{|c|c|c|c|}
\hline Treatment component & $\begin{array}{l}\text { Best-practice advice } \\
\text { only (N received best- } \\
\text { practice advice }=162) \\
n(\%)^{\mathrm{a}}\end{array}$ & $\begin{array}{l}\text { Injection plus } \\
\text { best-practice } \\
\text { advice (N received } \\
\text { best-practice } \\
\text { advice = 162), n (\%) }\end{array}$ & $\begin{array}{l}\text { Total ( } N \text { received } \\
\text { best-practice } \\
\text { advice }=324) \text {, } \\
n(\%)^{\mathrm{a}}\end{array}$ \\
\hline Exercises prescribed & $162(100)$ & $162(100)$ & $324(100)$ \\
\hline Advice/information booklet provided & $161(99)$ & $162(100)$ & $323(100)$ \\
\hline Exercise diary issued & $162(100)$ & $159(98)$ & 321 (99) \\
\hline Action planner completed & $160(99)$ & $160(99)$ & $320(99)$ \\
\hline Exercise video online/DVD provided & $160(99)$ & $158(98)$ & $318(98)$ \\
\hline \multicolumn{4}{|l|}{ Exercise $^{b}$} \\
\hline $\begin{array}{l}1 \text { (a) Shoulder abduction supported } \\
\text { by table in sitting }\end{array}$ & $31(10)$ & $20(6)$ & $51(8)$ \\
\hline $\begin{array}{l}\text { (b) Shoulder flexion supported by } \\
\text { table in sitting }\end{array}$ & $22(7)$ & $18(6)$ & $40(6)$ \\
\hline $\begin{array}{l}\text { (a) Isometric shoulder external } \\
\text { rotation }\end{array}$ & $29(9)$ & $30(9)$ & $59(9)$ \\
\hline (b) Isometric shoulder abduction & $36(11)$ & $35(11)$ & $71(11)$ \\
\hline $\begin{array}{l}\text { (a) Resisted shoulder external } \\
\text { rotation: } 0 \text { degrees }\end{array}$ & $89(28)$ & $82(26)$ & $171(27)$ \\
\hline (b) Shoulder flexion up a wall & $60(19)$ & $66(21)$ & $126(20)$ \\
\hline $\begin{array}{l}\text { (a) Resisted shoulder external } \\
\text { rotation: } 90 \text { degrees }\end{array}$ & $16(5)$ & $32(10)$ & $48(8)$ \\
\hline (b) Shoulder raise using a weight & $26(8)$ & $30(9)$ & $56(9)$ \\
\hline $\begin{array}{l}5 \text { (a) Resisted shoulder abduction/ } \\
\text { external rotation }{ }^{c}\end{array}$ & $11(3)$ & $7(2)$ & $18(3)$ \\
\hline Total & $320(100)$ & $320(100)$ & $640(100)$ \\
\hline \multicolumn{4}{|c|}{$\begin{array}{l}\text { a Per cent of those who attended best-practice advice. } \\
\text { b Number of times exercise prescribed. Number of participants attending }=324 \text {, but participants usually received } \\
\text { more than one exercise. } \\
\text { c Using resistance band. }\end{array}$} \\
\hline
\end{tabular}

were prescribed one set of exercises, $74 \%$ (247/332) were prescribed up to eight repetitions and $83 \%$ (277/332) were requested to perform at a load of 3-4 on the Borg scale. Note that 55\% (184/332) of participants were prescribed all of these components correctly in combination and in accordance with the protocol. Where the protocol was not adhered to, clinicians nearly always overprescribed by including additional sets and/or repetitions, or by setting higher intensities with target Borg scale scores of $>4$. Only $3 \%(10 / 332)$ of participants were underprescribed at the initial session, with all of these relating to lower-target Borg scale scores.

\section{Exercise progression}

Appendix 4, Table 38, describes the proportion of participants who were progressed, maintained or regressed, as defined previously in Chapter 3. Two-thirds (227/339) of participants who received the progressive-exercise intervention had their exercise progressed in accordance with the instructions provided in training. A small minority of participants had their exercise programme regressed (39/339, $12 \%)$ overall, whereas the remaining participants maintained $(73 / 339 ; 22 \%)$ exercising at the same level as the initial session over the course of their treatment. 
TABLE 11 Content of progressive-exercise intervention

\begin{tabular}{|c|c|c|c|c|c|c|c|}
\hline Session ${ }^{a}$ & Intervention & $\begin{array}{l}\text { Attended, } \\
n(\%)\end{array}$ & $\begin{array}{l}\text { Exercises } \\
\text { prescribed, } \\
\mathbf{n}(\%)\end{array}$ & $\begin{array}{l}\text { Advice/information } \\
\text { booklet provided, } \\
\text { n (\%) }\end{array}$ & $\begin{array}{l}\text { Exercise diary } \\
\text { issued/reviewed, } \\
\mathbf{n}(\%)\end{array}$ & $\begin{array}{l}\text { Action planner } \\
\text { completed/ } \\
\text { reviewed, } n \text { (\%) }\end{array}$ & $\begin{array}{l}\text { Confidence ruler } \\
\text { reviewed, } \boldsymbol{n}(\%)\end{array}$ \\
\hline \multirow[t]{2}{*}{1} & Progressive exercise only & $167(100)$ & $167(100)$ & 166 (99) & $164(98)$ & $163(98)$ & 165 (99) \\
\hline & Injection plus progressive exercise & $172(100)$ & $172(100)$ & $171(99)$ & $171(99)$ & $172(100)$ & $172(100)$ \\
\hline \multirow[t]{2}{*}{2} & Progressive exercise only & $161(100)$ & $160(99)$ & $30(19)$ & $153(95)$ & $91(57)$ & $150(93)$ \\
\hline & Injection plus progressive exercise & $160(100)$ & $158(99)$ & $28(18)$ & $147(92)$ & $99(62)$ & $144(90)$ \\
\hline \multirow[t]{2}{*}{3} & Progressive exercise only & $144(100)$ & $138(96)$ & $23(16)$ & $133(92)$ & $88(61)$ & $124(86)$ \\
\hline & Injection plus progressive exercise & $136(100)$ & $132(97)$ & $25(18)$ & $126(93)$ & $88(65)$ & $117(86)$ \\
\hline \multirow[t]{2}{*}{4} & Progressive exercise only & $101(100)$ & $96(95)$ & $22(22)$ & $94(93)$ & $69(68)$ & $88(87)$ \\
\hline & Injection plus progressive exercise & $100(100)$ & $94(94)$ & 19 (19) & $93(93)$ & $67(67)$ & $88(88)$ \\
\hline \multirow[t]{2}{*}{5} & Progressive exercise only & $72(100)$ & $66(92)$ & $16(22)$ & $66(92)$ & $52(72)$ & $62(86)$ \\
\hline & Injection plus progressive exercise & $69(100)$ & $66(96)$ & $11(16)$ & $64(93)$ & $49(71)$ & $62(90)$ \\
\hline \multirow[t]{2}{*}{6} & Progressive exercise only & $44(100)$ & $42(95)$ & $14(32)$ & $38(86)$ & $31(70)$ & $37(84)$ \\
\hline & Injection plus progressive exercise & $38(100)$ & $36(95)$ & $4(11)$ & $35(92)$ & $25(66)$ & $34(89)$ \\
\hline
\end{tabular}

a Percentage of those who attended session. 


\section{Participant-reported adherence to exercise}

As part of the participant follow-up questionnaires, participants were asked whether or not they were currently performing the exercises the physiotherapist asked them to complete at home as part of the GRASP trial. A total of $77.8 \%$ of participants reported 'yes' at 8 weeks, but this dropped to $49.3 \%$ and $32.1 \%$ at 6 and 12 months, respectively. Participants were also asked how often they were performing these exercises. Appendix 4, Table 39, shows the difference in proportions across intervention groups. At 8 weeks, participants who received progressive exercise were more likely to report performing their exercises 5 days per week than those receiving best-practice advice $(60.4 \%$ vs. $43.2 \%)$ and there was little difference at 6 and 12 months. At 8 weeks and 6 months, participants who received injection were more likely to report performing their exercises 5 days per week than those who received no injection ( 8 weeks, $57.5 \%$ vs. $46.0 \%$; 6 months, $21.4 \%$ vs. $12.1 \%$ ). There was little difference at 12 months.

\section{Quality assurance}

As well as monitoring intervention delivery using the treatment logs, annual site visits using a standardised checklist to audit adherence to the GRASP trial protocol were conducted to evaluate the delivery of the interventions. A total of 48 visits involving 45 physiotherapists took place during the trial at 19 of the 20 trial sites. One site was not visited because of the small numbers recruited $(n=4)$. Observation of the treatment sessions indicated that all physiotherapists were following the protocol satisfactorily. Only two physiotherapists swapped treatment groups during the trial, because of staffing issues, and, therefore, delivered both exercise interventions. Feedback regarding suggested areas of improvement was provided after the treatment session. In general, the most common areas discussed were related to improving the assessment of potential barriers to participants' performance of the home exercise programme, linking the performance of the exercises with relevant functional goals and attempting to involve the participant more in the exercise prescription process.

\section{Outcomes and estimations}

The primary analysis was ITT and was based on all participants with at least one follow-up time point SPADI outcome and the baseline variables used in the model. From the 708 participants randomised, primary outcome data were available for $90.1 \%(n=638)$ of participants at 8 weeks, $86.9 \%(n=615)$ of participants at 6 months and $87.1 \%(n=617)$ of participants at 12 months. A total of 1869 participant data points contributed to the primary outcome-adjusted analysis model. The number of participants contributing data to each of the secondary outcomes are presented separately.

\section{Primary outcome: shoulder pain and function - SPADI}

The primary outcome was shoulder pain and function measured using the SPADI scale over 12 months. There was a considerable overall improvement in SPADI score in each of the four intervention groups from baseline over time. Overall, this represents a 32.2 (SD 23.9)-point improvement on the SPADI scale [overall SPADI score 54.1 (SD 18.4) at baseline and 21.9 (SD 23.4) at 12 months] (Table 12). There is no substantial evidence of a floor or ceiling effect in SPADI score, which is considered to be present if $>15 \%$ of the respondents achieved the lowest or highest possible score, respectively. ${ }^{83}$

\section{Interaction}

As per the SAP, ${ }^{37}$ the presence of an interaction on the primary outcome was formally investigated. $A$ repeated-measures linear mixed-effects regression model was fitted to test the presence of an 
TABLE 12 Shoulder Pain and Disability Index summary at each time point by randomised group

\begin{tabular}{llllll}
$\begin{array}{l}\text { Time } \\
\text { point }\end{array}$ & $\begin{array}{l}\text { Best-practice } \\
\text { advice }(\mathbf{N}=174)\end{array}$ & $\begin{array}{l}\text { Injection plus } \\
\text { best-practice } \\
\text { advice }(\mathbf{N}=178)\end{array}$ & $\begin{array}{l}\text { Progressive } \\
\text { exercise }(\mathbf{N}=174)\end{array}$ & $\begin{array}{l}\text { Injection plus } \\
\text { progressive } \\
\text { exercise }(\mathbf{N}=182)\end{array}$ & $\begin{array}{l}\text { Overall } \\
(\mathbf{N}=\mathbf{7 0 8})\end{array}$ \\
Baseline & $57.0(19.0), 174$ & $55.3(19.0), 178$ & $50.5(17.4), 172$ & $53.6(17.8), 182$ & $54.1(18.5), 706$ \\
8 weeks & $43.8(22.6), 149$ & $33.5(21.6), 163$ & $39.7(21.6), 157$ & $37.9(24.0), 169$ & $38.6(22.8), 638$ \\
6 months & $28.3(24.4), 143$ & $28.1(23.4), 158$ & $24.1(22.2), 151$ & $26.6(24.0), 163$ & $26.8(23.6), 615$ \\
12 months & $24.0(24.8), 143$ & $23.9(23.5), 160$ & $17.9(21.1), 153$ & $21.7(24.0), 161$ & $21.9(23.4), 617$ \\
\hline $\begin{array}{l}\text { Note } \\
\text { Values are mean (SD), } n .\end{array}$ & & & & &
\end{tabular}

interaction between injection and progressive exercise. The model was adjusted for the fixed effects of age (continuous value), sex, baseline SPADI and time point, with random intercepts by centre, physiotherapist and observations within participant. The physiotherapist treating the largest number of sessions was selected for each participant and included in the model. The interaction coefficient between progressive exercise and injection over 12 months was $2.17(95 \% \mathrm{Cl}-2.96$ to $7.31 ; p=0.407)$, showing no evidence of a statistically significant interaction effect. Additionally, the interaction was assessed at each time point using a regression model adjusted for similar variables. At 8 weeks, the interaction was $3.41(95 \% \mathrm{Cl}-3.24$ to $10.06 ; p=0.314)$, at 6 months the interaction was $-1.88(95 \% \mathrm{Cl}$ -9.98 to $6.22 ; p=0.649)$ and at 12 months the interaction was $2.25(95 \% \mathrm{Cl}-5.76$ to $10.26 ; p=0.581)$, showing no evidence of a statistically significant effect. As per the SAP, the analysis at the margins is, therefore, the primary analysis for interpretation (i.e. considering the main effects of injection vs. no injection and progressive exercise vs. best-practice advice). Inside-the-table analysis results are reported in Appendix 4, Tables 40 and 41, for completeness.

The results presented here are for the two main effect comparisons:

1. an individually tailored progressive-exercise programme compared with a best-practice advice session with a physiotherapist supported by high-quality materials

2. subacromial corticosteroid injection compared with no injection.

\section{SPADI: progressive exercise compared with best-practice advice}

Table 13 presents the unadjusted and adjusted results for SPADI at 8 weeks and at 6 and 12 months. Figure 4 shows an improvement in shoulder pain and function in both intervention groups over time. However, there was no evidence of a statistically significant difference between the progressiveexercise intervention and best-practice advice intervention in shoulder pain and function when analysed over 12 months (adjusted mean SPADI difference between groups $-0.66,99 \% \mathrm{Cl}-4.52$ to 3.20). There was also no statistically significant difference between progressive exercise and bestpractice advice when analysed at 8 weeks and at 6 at 12 months (adjusted MD at 12 months -3.10 , $99 \% \mathrm{Cl}-7.85$ to 1.64$)$.

\section{SPADI: injection compared with no injection}

Table 14 presents the unadjusted and adjusted results for SPADI at 8 weeks and at 6 and 12 months. Figure 5 shows an improvement in shoulder pain and function in both groups over time. Injection resulted in an improvement in shoulder pain and function when analysed at 8 weeks compared with no injection (adjusted mean SPADI difference between groups $-5.64,99 \% \mathrm{Cl}-9.93$ to -1.35 ). 
TABLE 13 Progressive exercise vs. best-practice advice at-the-margins analysis of SPADI

\begin{tabular}{|c|c|c|c|c|c|c|c|c|c|c|}
\hline \multirow[b]{2}{*}{ SPADI } & \multirow[b]{2}{*}{$n$} & \multicolumn{3}{|c|}{ Best-practice advice } & \multicolumn{4}{|c|}{ Progressive exercise } & \multirow{2}{*}{$\begin{array}{l}\text { Between- } \\
\text { group adjusted } \\
\text { difference } \\
(99 \% \mathrm{CI})\end{array}$} & \multirow[b]{2}{*}{$p$-value ${ }^{a}$} \\
\hline & & $\begin{array}{l}\text { Unadjusted } \\
\text { mean (SD) }\end{array}$ & $n$ & $\begin{array}{l}\text { Adjusted } \\
\text { mean (SE) }\end{array}$ & $n$ & $\begin{array}{l}\text { Unadjusted } \\
\text { mean (SD) }\end{array}$ & $n$ & $\begin{array}{l}\text { Adjusted } \\
\text { mean (SE) }\end{array}$ & & \\
\hline Baseline & 352 & $56.1(19)$ & & & 354 & $52.1(17.7)$ & & & & \\
\hline 8 weeks & 312 & $38.4(22.7)$ & 297 & $36.96(1.32)$ & 326 & $38.7(22.9)$ & 316 & $39.50(1.26)$ & $\begin{array}{l}2.54 \\
(-2.16 \text { to } 7.23)\end{array}$ & 0.164 \\
\hline 6 months & 301 & $28.2(23.8)$ & 288 & $27.39(1.33)$ & 314 & $25.4(23.1)$ & 307 & $25.87(1.27)$ & $\begin{array}{l}-1.52 \\
(-6.26 \text { to } 3.22)\end{array}$ & 0.410 \\
\hline 12 months & 303 & $24.0(24.1)$ & 288 & $23.67(1.33)$ & 314 & $19.9(22.7)$ & 307 & $20.57(1.27)$ & $\begin{array}{l}-3.10 \\
(-7.85 \text { to } 1.64)\end{array}$ & 0.092 \\
\hline $\begin{array}{l}\text { Over } \\
12 \text { months }\end{array}$ & 339 & $30.3(24.3)$ & 339 & $29.41(1.08)$ & 343 & $28.1(24.2)$ & 343 & $28.75(1.03)$ & $\begin{array}{l}-0.66 \\
(-4.52 \text { to } 3.20)\end{array}$ & 0.659 \\
\hline $\begin{array}{l}\text { CACE } \\
\text { estimate }^{b}\end{array}$ & & & & & & & & & $\begin{array}{l}-0.27 \\
(-2.69 \text { to } 2.16)\end{array}$ & 0.830 \\
\hline
\end{tabular}

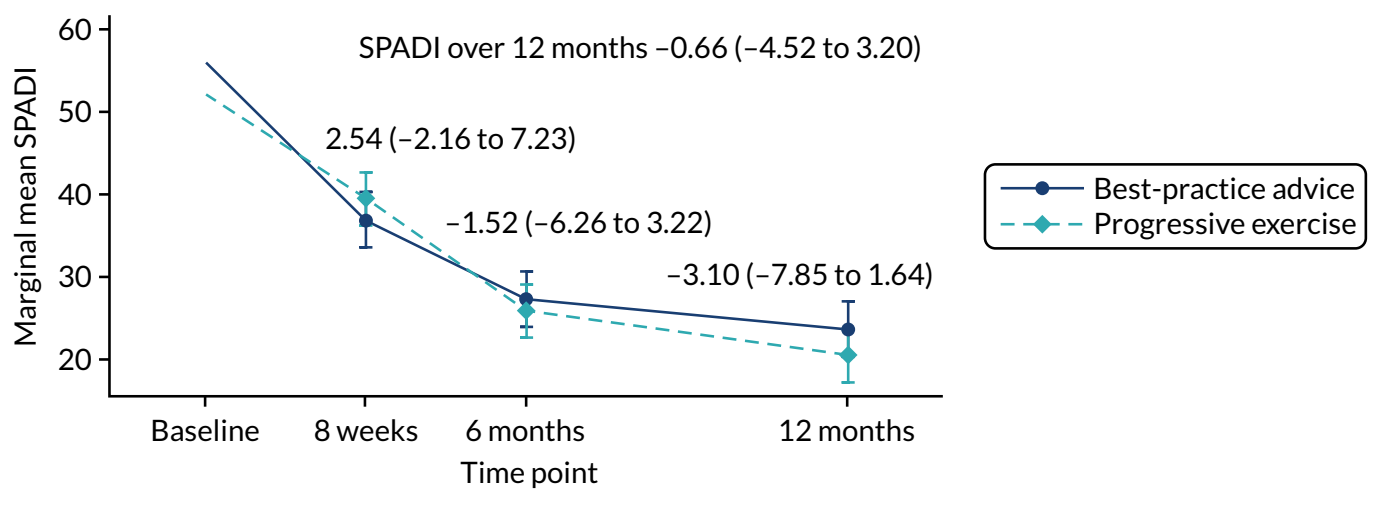

FIGURE 4 Marginal adjusted mean SPADI values from the repeated measures mixed-effects model and associated $99 \% \mathrm{Cls}$ for the two treatment groups from baseline to 12 months: best-practice advice vs. progressive exercise.

TABLE 14 Injection vs. no injection at-the-margins analysis of SPADI

\begin{tabular}{|c|c|c|c|c|c|c|c|c|c|c|}
\hline \multirow[b]{2}{*}{ SPADI } & \multicolumn{4}{|c|}{ No injection } & \multicolumn{4}{|c|}{ Injection } & \multirow{2}{*}{$\begin{array}{l}\text { Between- } \\
\text { group adjusted } \\
\text { difference } \\
\text { (99\% CI) }\end{array}$} & \multirow[b]{2}{*}{$p$-value ${ }^{a}$} \\
\hline & $n$ & $\begin{array}{l}\text { Unadjusted } \\
\text { mean (SD) }\end{array}$ & $n$ & $\begin{array}{l}\text { Adjusted } \\
\text { mean (SE) }\end{array}$ & $n$ & $\begin{array}{l}\text { Unadjusted } \\
\text { mean (SD) }\end{array}$ & $n$ & $\begin{array}{l}\text { Adjusted } \\
\text { mean (SE) }\end{array}$ & & \\
\hline Baseline & 346 & $53.8(18.6)$ & & & 360 & $54.4(18.4)$ & & & & \\
\hline 8 weeks & 306 & $41.7(22.2)$ & 300 & $41.16(1.24)$ & 332 & 35.7 (22.9) & 313 & $35.52(1.22)$ & $\begin{array}{l}-5.64 \\
(-9.93 \text { to }-1.35)\end{array}$ & 0.001 \\
\hline 6 months & 294 & $26.2(23.4)$ & 289 & $26.33(1.26)$ & 321 & $27.3(23.7)$ & 306 & 26.85 & $\begin{array}{l}0.52 \\
(-3.82 \text { to } 4.86)\end{array}$ & 0.758 \\
\hline 12 months & 296 & $20.9(23.1)$ & 290 & $21.07(1.26)$ & 321 & $22.8(23.7)$ & 305 & $23.00(1.23)$ & $\begin{array}{l}1.93 \\
(-2.41 \text { to } 6.27)\end{array}$ & 0.251 \\
\hline $\begin{array}{l}\text { Over } \\
12 \text { months }\end{array}$ & 330 & $29.7(24.5)$ & 330 & $29.63(1.0)$ & 352 & $28.7(24.0)$ & 352 & $28.53(0.98)$ & $\begin{array}{l}-1.11 \\
(-4.47 \text { to } 2.26)\end{array}$ & 0.397 \\
\hline $\begin{array}{l}\text { CACE } \\
\text { estimate }^{\text {b }}\end{array}$ & & & & & & & & & $\begin{array}{l}-1.50 \\
(-3.61 \text { to } 0.61)\end{array}$ & 0.164 \\
\hline
\end{tabular}

a SPADI analysis adjusted for age, sex and baseline SPADI, with random effects within participant, physiotherapist and centre. b $95 \% \mathrm{Cl}$ presented. 


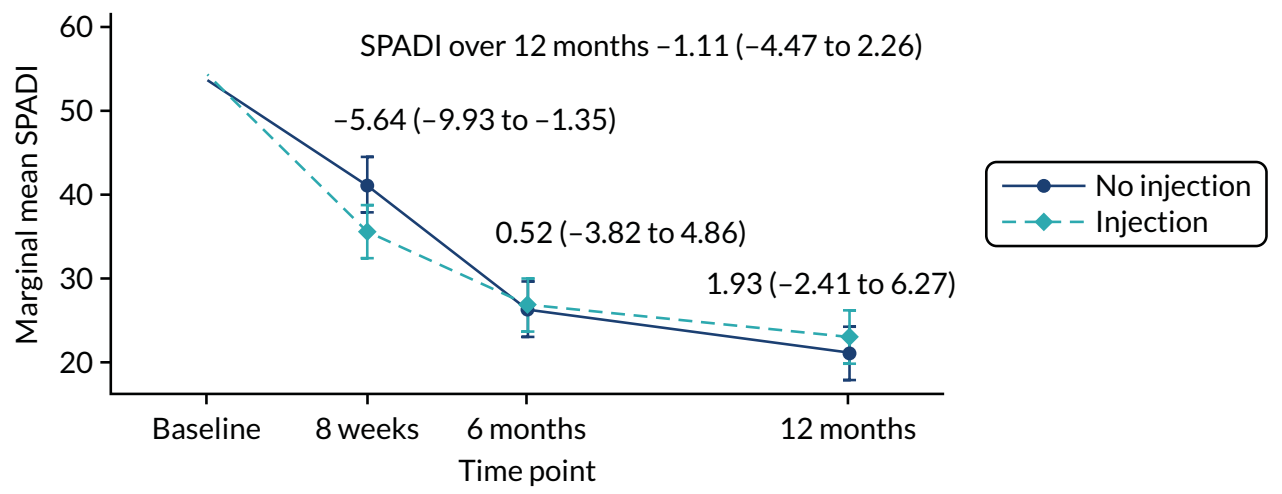

FIGURE 5 Marginal adjusted mean SPADI values from the repeated measures mixed-effects model and associated $99 \% \mathrm{Cls}$ for the two treatment groups from baseline to 12 months: injection vs. no injection.

This difference was not deemed clinically significant where the MCID on the SPADI scale is 8 points on a scale of $0-100$. There was no statistically significant difference between injection and no injection when analysed over 12 months (adjusted MD $-1.11,99 \% \mathrm{Cl}-4.47$ to 2.26 ) or when analysed at 6 and 12 months (adjusted MD at 12 months $1.93,99 \% \mathrm{Cl}-2.41$ to 6.27 ).

\section{Sensitivity analysis}

\section{Complier-average causal effect analysis}

The primary outcome was analysed in the ITT population. An additional CACE analysis was used to investigate the role of compliance with intervention on the trial effects for the primary outcome. Compliance with intervention was defined in the $\mathrm{SAP}^{37}$ as follows. For the progressive-exercise intervention, participants were considered compliant with treatment if they had been signed off for completing treatment or if they received all six physiotherapy sessions. For the injection intervention, participants were considered compliant if they received at least one injection. Compliance with treatment by intervention group is summarised in Table 8. As no evidence of a statistical interaction effect between interventions was identified, the CACE analysis was conducted at the margins for the two main effect comparisons.

\section{Progressive exercise compared with best-practice advice}

The proportion of compliers in the progressive-exercise intervention group was $78 \%$. Compliance with the progressive-exercise intervention did not have a significant effect on the primary outcome. The CACE estimate analysis for progressive exercise over 12 months was (mean adjusted difference) -0.27 (95\% Cl -2.69 to 2.16; $p=0.830$ ) (see Table 13). The model included 1869 participant data points and was adjusted for sex, age, baseline SPADI and centre. The variable for primary physiotherapist was omitted from this analysis because of collinearity.

\section{Injection compared with no injection}

The proportion of compliers in the injection treatment group was $94 \%$. Compliance with injection did not have a significant effect on the primary outcome. The CACE estimate analysis for injection over 12 months was mean adjusted difference $-1.50(95 \% \mathrm{Cl}-3.61$ to $0.61 ; p=0.164)$ (see Table 14). The model included 1869 participant data points and was adjusted for sex, age, baseline SPADI and centre. The variable for primary physiotherapist was, again, omitted because of collinearity.

\section{Secondary outcomes}

The results presented here for secondary outcomes are for the two main effect comparisons. 


\section{Progressive exercise compared with best-practice advice}

Table 15 presents the adjusted analysis results for the comparison of progressive exercise with best-practice advice for each of the secondary outcomes at 8 weeks and at 6 and 12 months (for unadjusted results see Appendix 4, Table 42).

TABLE 15 Analysis of secondary outcomes for progressive exercise vs. best-practice advice at 8 weeks, 6 months and 12 months

\begin{tabular}{|c|c|c|c|c|c|c|}
\hline \multirow[b]{2}{*}{ Secondary outcome } & \multicolumn{2}{|c|}{ Best-practice advice } & \multicolumn{2}{|c|}{ Progressive exercise } & \multirow{2}{*}{$\begin{array}{l}\text { Between-group } \\
\text { adjusted difference } \\
\text { (95\% CI) }\end{array}$} & \multirow[b]{2}{*}{$p$-value ${ }^{a}$} \\
\hline & $n$ & Adjusted mean (SE) & $n$ & Adjusted mean (SE) & & \\
\hline
\end{tabular}

SPADI: pain

8 weeks

6 months

12 months

Over 12 months

$\begin{array}{ll}300 & 49.9(1.4) \\ 289 & 32.0(1.4) \\ 290 & 25.8(1.4) \\ 339 & 36.0(1.3)\end{array}$

SPADI: function

$\begin{array}{lll}8 \text { weeks } & 298 & 29.40(1.24) \\ 6 \text { months } & 288 & 20.95(1.26) \\ 12 \text { months } & 288 & 18.74(1.26) \\ \text { Over 12 months } & 339 & 23.1(1.0)\end{array}$

FABQ-PA

8 weeks

6 months

$269 \quad 11.62(0.37)$

12 months

Over 12 months

266

$9.93(0.37)$

$2669.29(0.37)$

$324 \quad 10.3(0.30)$

PSEQ-2

$\begin{array}{lll}8 \text { weeks } & 271 & 10.25(0.13) \\ 6 \text { months } & 267 & 10.41(0.13) \\ 12 \text { months } & 267 & 10.59(0.13) \\ \text { Over 12 months } & 325 & 10.41(0.10)\end{array}$

ISI

$\begin{array}{lllll}8 \text { weeks } & 267 & 7.46(0.32) & 294 & 8.09(0.31) \\ 6 \text { months } & 264 & 6.20(0.32) & 281 & 6.20(0.31) \\ 12 \text { months } & 267 & 5.92(0.32) & 282 & 5.40(0.31) \\ \text { Over 12 months } & 323 & 6.53(0.27) & 329 & 6.57(0.26)\end{array}$
RDA

\begin{tabular}{lllllll}
8 weeks & 270 & $6.08(0.14)$ & 297 & $6.33(0.13)$ & $0.25(-0.12$ to 0.62$)$ & 0.189 \\
6 months & 267 & $5.42(0.14)$ & 284 & $5.10(0.14)$ & $-0.31(-0.69$ to 0.06$)$ & 0.101 \\
12 months & 268 & $4.81(0.14)$ & 285 & $4.67(0.14)$ & $-0.14(-0.51$ to 0.24$)$ & 0.466 \\
Over 12 months & 325 & $5.44(0.11)$ & 332 & $5.38(0.11)$ & $-0.06(-0.36$ to 0.23$)$ & 0.662 \\
\hline
\end{tabular}


TABLE 15 Analysis of secondary outcomes for progressive exercise vs. best-practice advice at 8 weeks, 6 months and 12 months (continued)

\begin{tabular}{|c|c|c|c|c|c|c|}
\hline \multirow[b]{2}{*}{ Secondary outcome } & \multicolumn{2}{|c|}{ Best-practice advice } & \multicolumn{2}{|c|}{ Progressive exercise } & \multirow{2}{*}{$\begin{array}{l}\text { Between-group } \\
\text { adjusted difference } \\
(95 \% \mathrm{CI})\end{array}$} & \multirow[b]{2}{*}{$p$-value } \\
\hline & n & Adjusted mean (SE) & $n$ & Adjusted mean (SE) & & \\
\hline \multicolumn{7}{|l|}{ GIT } \\
\hline 8 weeks & 269 & $7.65(0.13)$ & 298 & $7.75(0.13)$ & $0.11(-0.25$ to 0.47$)$ & 0.555 \\
\hline 6 months & 267 & $8.16(0.13)$ & 285 & $8.69(0.13)$ & 0.53 (0.17 to 0.90$)$ & 0.004 \\
\hline 12 months & 270 & $8.57(0.13)$ & 286 & $9.08(0.13)$ & 0.51 (0.14 to 0.87$)$ & 0.006 \\
\hline Over 12 months & 326 & $8.12(0.10)$ & 332 & $8.50(0.10)$ & 0.38 (0.10 to 0.66$)$ & 0.007 \\
\hline
\end{tabular}

a Outcome analysis adjusted for age, sex and baseline outcome value, with random effects within participant, physiotherapist and centre.

\section{SPADI: shoulder pain subscale}

There was an improvement in shoulder pain in both intervention groups over time (Figure 6). However, there was no statistically significant difference between the progressive-exercise intervention and bestpractice advice in shoulder pain when analysed over 12 months (adjusted mean SPADI pain subscale difference between groups $-1.61,95 \% \mathrm{Cl}-4.94$ to 1.72) or when analysed at 8 weeks or at 6 and 12 months (adjusted MD at 12 months $-4.01,95 \% \mathrm{Cl}-8.11$ to 0.09 ).

\section{SPADI: shoulder function subscale}

There was an improvement in shoulder function in both intervention groups over time (Figure 7). However, there was no statistically significant difference between the progressive-exercise intervention and best-practice advice in shoulder function when analysed over 12 months (adjusted mean SPADI function subscale difference between groups $-0.15,95 \% \mathrm{Cl}-2.92$ to 2.61 ) or when analysed at 8 weeks or at 6 and 12 months (adjusted MD at 12 months $-2.61,95 \% \mathrm{Cl}-6.03$ to 0.81 ).

\section{Fear Avoidance Belief Questionnaire - Physical Activity}

There was an improvement in fear avoidance behaviour in both intervention groups over time. However, there was no statistically significant difference between the progressive-exercise intervention and the best-practice advice intervention in fear avoidance behaviour when analysed over 12 months (adjusted mean FABQ-PA score difference between groups $-0.35,95 \% \mathrm{Cl}-1.16$ to 0.46 ) or when analysed at 8 weeks, 6 months or 12 months (adjusted MD at 12 months $-0.97,95 \% \mathrm{Cl}-1.99$ to 0.05 ).

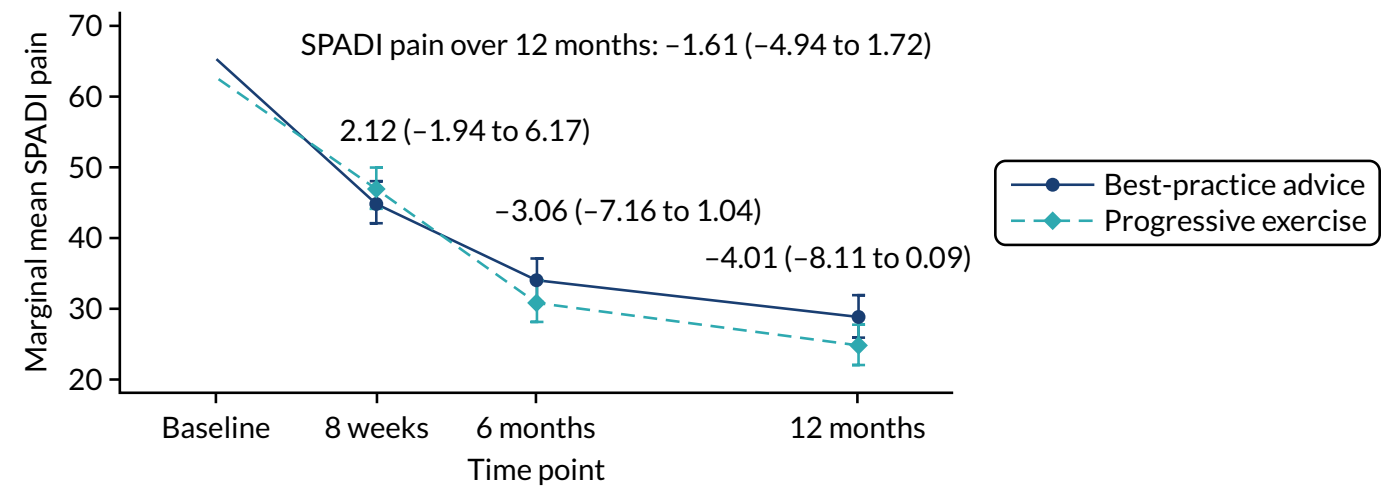

FIGURE 6 Marginal adjusted mean SPADI pain values from the repeated measures mixed-effects model and associated 95\% Cls for the two treatment groups from baseline to 12 months: best-practice advice vs. progressive exercise. 


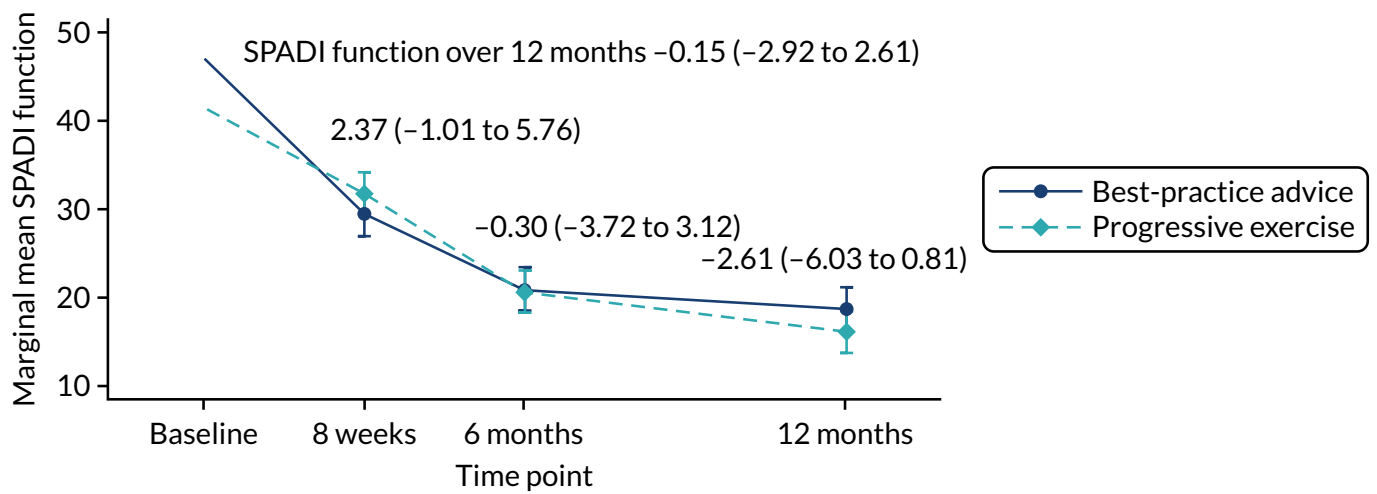

FIGURE 7 Marginal adjusted mean SPADI function values from the repeated measures mixed-effects model and associated 95\% Cls for the two treatment groups from baseline to 12 months: best-practice advice vs. progressive exercise.

\section{Pain Self-Efficacy Questionnaire}

There was no statistically significant difference between the progressive-exercise intervention and best-practice advice intervention in patient-reported pain self-efficacy when analysed over 12 months (adjusted mean PSEQ-2 score difference between groups $0.02,95 \% \mathrm{Cl}-0.23$ to 0.27 ) or when analysed at 8 weeks, 6 months or 12 months (adjusted MD at 12 months $0.12,95 \% \mathrm{Cl}-0.22$ to 0.45 ). A ceiling effect was noted for this outcome, with most participants reporting higher confidence levels despite experiencing pain.

\section{Insomnia Severity Index}

There was an improvement in patient-reported perception of insomnia in both intervention groups over time. However, there was no statistically significant difference between the progressive-exercise intervention and best-practice advice in patient-reported perception of insomnia when analysed over 12 months (adjusted mean ISI difference between groups $0.04,95 \% \mathrm{Cl}-0.69$ to 0.77 ) or when analysed at 8 weeks, 6 months or 12 months (adjusted MD at 12 months $-0.52,95 \% \mathrm{Cl}-1.40$ to 0.36 ).

\section{Return to desired activities}

There was an improvement in patient-reported RDA in both intervention groups over time. However, there was no statistically significant difference between the progressive-exercise intervention and bestpractice advice intervention in patient-reported RDA when analysed over 12 months (adjusted mean RDA difference between groups $-0.06,95 \% \mathrm{Cl}-0.36$ to 0.23 ) or when analysed at 8 weeks, 6 months or 12 months (adjusted MD at 12 months $-0.14,95 \% \mathrm{Cl}-0.51$ to 0.24 ).

\section{Global Impression of Treatment}

Progressive exercise resulted in an improvement in patient-reported GIT success over 12 months (adjusted mean GIT difference between groups $0.38,95 \% \mathrm{CI} 0.10$ to 0.66 ) and at 6 and 12 months (adjusted MD at 12 months $0.51,95 \% \mathrm{Cl} 0.14$ to 0.87 ), compared with best-practice advice. However, this result was not seen at 8 weeks and the difference was not clinically significant (MCID on the GIT scale is 2 points on an 11-point scale).

\section{Injection compared with no injection}

Table 16 presents the adjusted results for the comparison of injection with no injection for each of the secondary outcomes at 8 weeks, 6 months and 12 months (for unadjusted results, see Appendix 4, Table 42).

\section{SPADI: shoulder pain subscale}

There was an improvement in shoulder pain in both groups over time (Figure 8). Injection resulted in an improvement in shoulder pain when analysed at 8 weeks, compared with no injection (adjusted mean SPADI pain subscale difference between groups $-7.38,95 \% \mathrm{Cl}-11.10$ to -3.67$)$. This difference was 
TABLE 16 Analysis of secondary outcomes for injection vs. no injection at 8 weeks, 6 months and 12 months

\begin{tabular}{|c|c|c|c|c|}
\hline \multirow[b]{2}{*}{ Secondary outcome } & No injection & Injection & \multirow{2}{*}{$\begin{array}{l}\text { Between-group } \\
\text { adjusted difference } \\
\text { (95\% CI) }\end{array}$} & \multirow[b]{2}{*}{$p$-value ${ }^{a}$} \\
\hline & Adjusted mean (SE) & Adjusted mean (SE) & & \\
\hline
\end{tabular}

SPADI: pain

$\begin{array}{lll}8 \text { weeks } & 300 & 49.9(1.4) \\ 6 \text { months } & 289 & 32.0(1.4) \\ 12 \text { months } & 290 & 25.8(1.4) \\ \text { Over 12 months } & 339 & 36.0(1.3)\end{array}$

$\begin{array}{ll}315 & 42.9(1.4) \\ 306 & 32.9(1.4) \\ 306 & 27.8(1.4) \\ 343 & 34.5(1.1)\end{array}$

$\begin{array}{cc}-7.38(-11.10 \text { to }-3.67) & 0.000 \\ 0.89(-2.88 \text { to } 4.66) & 0.643 \\ 2.05(-1.72 \text { to } 5.81) & 0.286 \\ -1.55(-4.46 \text { to } 1.37) & 0.299\end{array}$

SPADI: function

$\begin{array}{lll}8 \text { weeks } & 301 & 32.59(1.18) \\ 6 \text { months } & 289 & 20.69(1.19) \\ 12 \text { months } & 290 & 16.38(1.19) \\ \text { Over 12 months } & 339 & 23.31(0.93)\end{array}$

$\begin{array}{ll}313 & 28.75(1.16) \\ 306 & 20.89(1.17) \\ 305 & 18.34(1.17) \\ 343 & 22.72(0.92)\end{array}$

$\begin{array}{ll}-3.84(-6.95 \text { to }-0.73) & 0.015 \\ 0.20(-2.95 \text { to } 3.35) & 0.900 \\ 1.97(-1.18 \text { to } 5.11) & 0.221 \\ -0.59(-3.02 \text { to } 1.83) & 0.631\end{array}$

FABQ-PA

$\begin{array}{llr}8 \text { weeks } & 274 & 12.02(0.36) \\ 6 \text { months } & 262 & 9.70(0.37) \\ 12 \text { months } & 265 & 8.55(0.37) \\ \text { Over 12 months } & 316 & 8.22(0.10)\end{array}$

$\begin{array}{lr}296 & 11.59(0.35) \\ 285 & 9.72(0.35) \\ 285 & 9.01(0.35) \\ 340 & 8.41(0.10)\end{array}$

$\begin{array}{cc}-0.43(-1.39 \text { to } 0.53) & 0.381 \\ 0.03(-0.95 \text { to } 1.01) & 0.954 \\ 0.47(-0.51 \text { to } 1.44) & 0.349 \\ 0.02(-0.74 \text { to } 0.77) & 0.967\end{array}$

PSEQ-2

$\begin{array}{lll}8 \text { weeks } & 275 & 10.13(0.13) \\ 6 \text { months } & 264 & 10.44(0.13) \\ 12 \text { months } & 266 & 10.80(0.13) \\ \text { Over 12 months } & 317 & 10.45(0.10)\end{array}$

$\begin{array}{ll}296 & 10.30(0.12) \\ 285 & 10.39(0.13) \\ 285 & 10.50(0.13) \\ 340 & 10.40(0.10)\end{array}$

$$
\begin{array}{rr}
0.17(-0.16 \text { to } 0.50) & 0.307 \\
-0.05 \text { (-0.38 to } 0.28) & 0.773 \\
-0.30 \text { (-0.63 to } 0.03) & 0.078 \\
-0.06(-0.31 \text { to } 0.19) & 0.659
\end{array}
$$

ISI

$\begin{array}{lll}8 \text { weeks } & 270 & 8.57(0.31) \\ 6 \text { months } & 262 & 6.21(0.31) \\ 12 \text { months } & 265 & 5.48(0.31) \\ \text { Over 12 months } & 314 & 6.77(0.25)\end{array}$

$\begin{array}{ll}291 & 7.07(0.30) \\ 283 & 6.18(0.30) \\ 284 & 5.80(0.30) \\ 338 & 6.35(0.25)\end{array}$

$-1.50(-2.32$ to -0.68$) \quad 0.000$

-0.03 (-0.86 to 0.80$) \quad 0.947$

0.32 (-0.51 to 1.15$) \quad 0.449$

-0.41 ( -1.08 to 0.26$) \quad 0.227$

RDA

$\begin{array}{lll}8 \text { weeks } & 273 & 6.49(0.14) \\ 6 \text { months } & 266 & 5.27(0.14) \\ 12 \text { months } & 268 & 4.63(0.14) \\ \text { Over 12 months } & 317 & 5.47(0.11)\end{array}$

$\begin{array}{ll}294 & 5.96(0.13) \\ 285 & 5.24(0.14) \\ 285 & 4.84(0.14) \\ 340 & 5.35(0.11)\end{array}$

$-0.53(-0.89$ to -0.17$) \quad 0.004$

$-0.03(-0.39$ to 0.34$) \quad 0.893$

0.21 (-0.15 to 0.57$) \quad 0.258$

$-0.12(-0.40$ to 0.16$) \quad 0.409$

GIT

$\begin{array}{lllllll}8 \text { weeks } & 273 & 7.34(0.13) & 294 & 8.03(0.13) & 0.69 \text { (0.35 to } 1.03) & 0.000 \\ 6 \text { months } & 267 & 8.42(0.13) & 285 & 8.45(0.13) & 0.04(-0.31 \text { to } 0.39) & 0.832 \\ \text { 12 months } & 269 & 8.91(0.13) & 287 & 8.76(0.13) & -0.14(-0.49 \text { to } 0.20) & 0.416 \\ \text { Over 12 months } & 317 & 8.22(0.10) & 347 & 8.41(0.10) & 0.20(-0.06 \text { to } 0.46) & 0.138\end{array}$

a Outcome analysis adjusted for age, sex and baseline outcome value, with random effects within participant, physiotherapist and centre. 


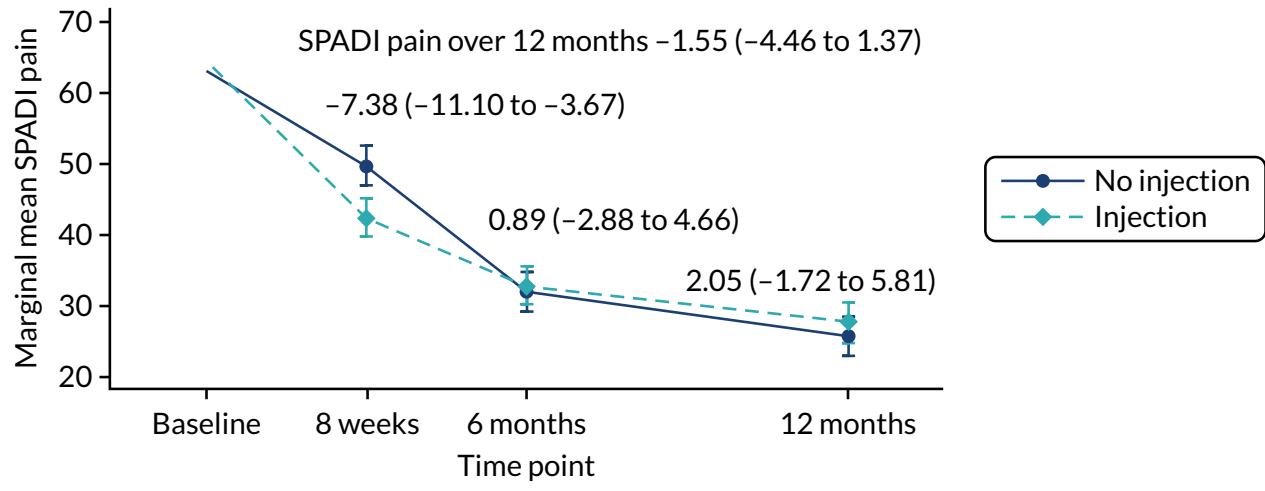

FIGURE 8 Marginal adjusted mean SPADI pain values from the repeated measures mixed-effects model and associated 95\% Cls for the two treatment groups from baseline to 12 months: injection vs. no injection.

not clinically significant (MCID on the SPADI scale is 8 points on a scale of 0-100). There was no statistically significant difference between injection and no injection when analysed over 12 months (adjusted MD over 12 months $-1.55,95 \% \mathrm{Cl}-4.46$ to 1.37) or when analysed at 6 and 12 months (adjusted $\mathrm{MD}$ at 12 months $2.05,95 \% \mathrm{Cl}-1.72$ to 5.81 ).

\section{SPADI: shoulder function subscale}

There was an improvement in shoulder function in both groups over time (Figure 9). Injection resulted in an improvement in shoulder function when analysed at 8 weeks compared with no injection (adjusted mean SPADI function subscale difference between groups $-3.84,95 \% \mathrm{Cl}-6.95$ to -0.73 ). This difference was not clinically significant. There was no statistically significant difference between injection and no injection when analysed over 12 months (adjusted MD over 12 months $-0.59,95 \% \mathrm{Cl}$ -3.02 to 1.83) or when analysed at 6 and 12 months (adjusted MD at 12 months $1.97,95 \% \mathrm{Cl}-1.18$ to 5.11 ).

\section{Fear Avoidance Belief Questionnaire - Physical Activity}

There was an improvement in fear avoidance behaviour in both groups over time. However, there was no statistically significant difference between injection and no injection in fear avoidance behaviour when analysed over 12 months (adjusted mean FABQ-PA score difference between groups over 12 months $0.02,95 \% \mathrm{Cl}-0.74$ to 0.77 ) or when analysed at 8 weeks, 6 months or 12 months (adjusted MD at 12 months $0.47,95 \% \mathrm{Cl}-0.51$ to 1.44 ).

\section{Pain Self-Efficacy Questionnaire}

There was no statistically significant difference between injection and no injection in patient-reported pain self-efficacy when analysed over 12 months (adjusted mean PSEQ-2 score difference between groups over 12 months $-0.06,95 \% \mathrm{Cl}-0.31$ to 0.19$)$ or when analysed at 8 weeks, 6 months or

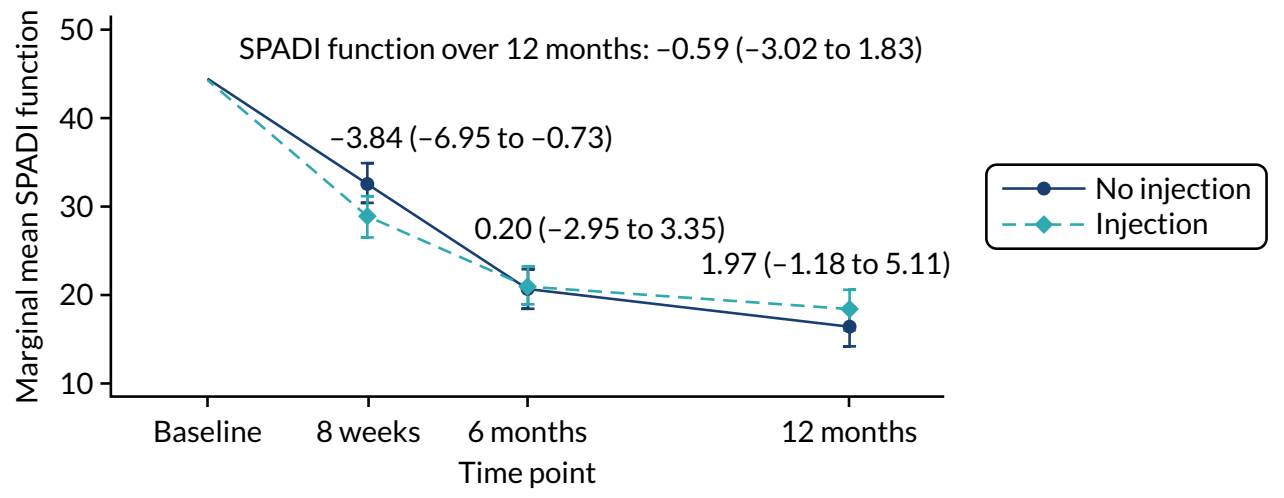

FIGURE 9 Marginal adjusted mean SPADI function values from the repeated measures mixed-effects model and associated $95 \% \mathrm{Cls}$ for the two treatment groups from baseline to 12 months: injection vs. no injection. 
12 months (adjusted MD at 12 months $-0.30,95 \% \mathrm{Cl}-0.63$ to 0.03 ). A ceiling effect was noted for this outcome, with most participants reporting higher confidence levels despite experiencing pain.

\section{Insomnia Severity Index}

There was an improvement in patient-reported perception of insomnia in both groups over time. Injection resulted in an improvement in patient-reported perception of insomnia when analysed at 8 weeks compared with no injection (adjusted mean ISI difference between groups $-1.50,95 \% \mathrm{CI}$ -2.32 to -0.68 ). There was no statistically significant difference between injection and no injection when analysed over 12 months (adjusted MD over 12 months $-0.41,95 \% \mathrm{Cl}-1.08$ to 0.26 ) or when analysed at 6 and 12 months (adjusted MD at 12 months $0.32,95 \% \mathrm{Cl}-0.51$ to 1.15 ).

\section{Return to desired activities}

There was an improvement in patient-reported RDA in both groups over time. Injection resulted in an improvement in patient-reported RDA when analysed at 8 weeks compared with no injection (adjusted mean RDA difference between groups $-0.53,95 \% \mathrm{Cl}-0.89$ to -0.17 ). There was no statistically significant difference between injection and no injection when analysed over 12 months (adjusted MD over 12 months $-0.12,95 \% \mathrm{Cl}-0.40$ to 0.16 ) or when analysed at 6 and 12 months (adjusted MD at 12 months $0.21,95 \% \mathrm{Cl}-0.15$ to 0.57$)$.

\section{Global Impression of Treatment}

Injection resulted in an improvement in patient-reported GIT success when analysed at 8 weeks compared with no injection (adjusted mean GIT difference between groups $0.69,95 \% \mathrm{Cl} 0.35$ to 1.03 ). There was no statistically significant difference between injection and no injection when analysed over 12 months (adjusted MD over 12 months $0.20,95 \% \mathrm{Cl}-0.06$ to 0.46 ) or when analysed at 6 and 12 months (adjusted $\mathrm{MD}$ at 12 months $-0.14,95 \% \mathrm{Cl}-0.49$ to 0.20 ).

\section{Other outcomes}

\section{Participant-reported shoulder condition}

As part of the follow-up questionnaires, a total of 23 (3\%) participants reported 'yes' to the question 'have you been told you will need to have surgery because of your shoulder problem'. Two, six and 15 participants reported 'yes' at 8 weeks, 6 months and 12 months, respectively, and the numbers were similar across intervention groups. Three participants reported that they had been admitted to hospital as an NHS inpatient because of their shoulder. The type of surgery recorded was surgery to repair rotator cuff tear ( $n=1$; injection plus progressive-exercise intervention), frozen shoulder surgery diagnosed after randomisation ( $n=1$; best-practice advice intervention), subacromial decompression and cuff repair ( $n=1$; injection plus best-practice advice intervention).

\section{Participant-reported injection outside the trial}

As part of the follow-up questionnaires, 16 (4\%) participants in the injection group and four (1\%) participants in the no injection group reported 'yes' to the question 'have you had a steroid injection as a result of pain in your shoulder?' at 8 weeks. Twelve (3\%) participants in the injection group and $18(5 \%)$ participants in the no injection group reported 'yes' to the question at 6 months, and $20(6 \%)$ participants in the injection group and six (2\%) participants in the no injection group reported 'yes' at 12 months. This excluded the injection(s) participants may have received as part of the GRASP trial.

\section{Harms}

There were no SAEs recorded as part of the GRASP trial. 


\section{Missing data}

There were small numbers of missing data. A total of 641 (90.5\%) participants returned the 8-week follow-up questionnaire, 615 (86.9\%) returned their 6 month-questionnaire and 618 (87.3\%) returned their 12-month questionnaire. Where items were missing within scales, these were dealt with based on published recommendations. SPADI score was imputed as per scoring instructions (see Chapter 2); eight (1.1\%) SPADI scores were imputed at baseline, six (0.8\%) SPADI scores were imputed at 8 weeks, four (0.6\%) SPADI scores were imputed at 6 months and two (0.3\%) SPADI scores were imputed at 12 months. Missing continuous data for primary and secondary outcomes were accounted for as part of the likelihood-based estimation of the repeated measures linear mixed-effects model, assuming that data were missing at random.

\section{Prespecified subgroup analysis}

The following prespecified subgroup analyses were carried out in line with the primary outcome analysis approach: age ( $\leq 64$ years $>65$ years), sex (male/female), smoking status (never smoked/former smoker or current smoker), higher SPADI score at baseline [SPADI $\geq 50$ at baseline (scale 0-100)] and higher pain self-efficacy score at baseline [PSEQ-2 $\geq 8$ at baseline (scale 0-12)]. The results presented here for prespecified subgroup analyses are for the two main effect comparisons.

\section{Progressive exercise compared with best-practice advice}

There were no statistically significant subgroup differences between the progressive-exercise intervention and best-practice advice intervention in shoulder pain and function when analysed over 12 months (Figure 10) or when analysed at 8 weeks, 6 months or 12 months (see Appendix 4, Figure 25).

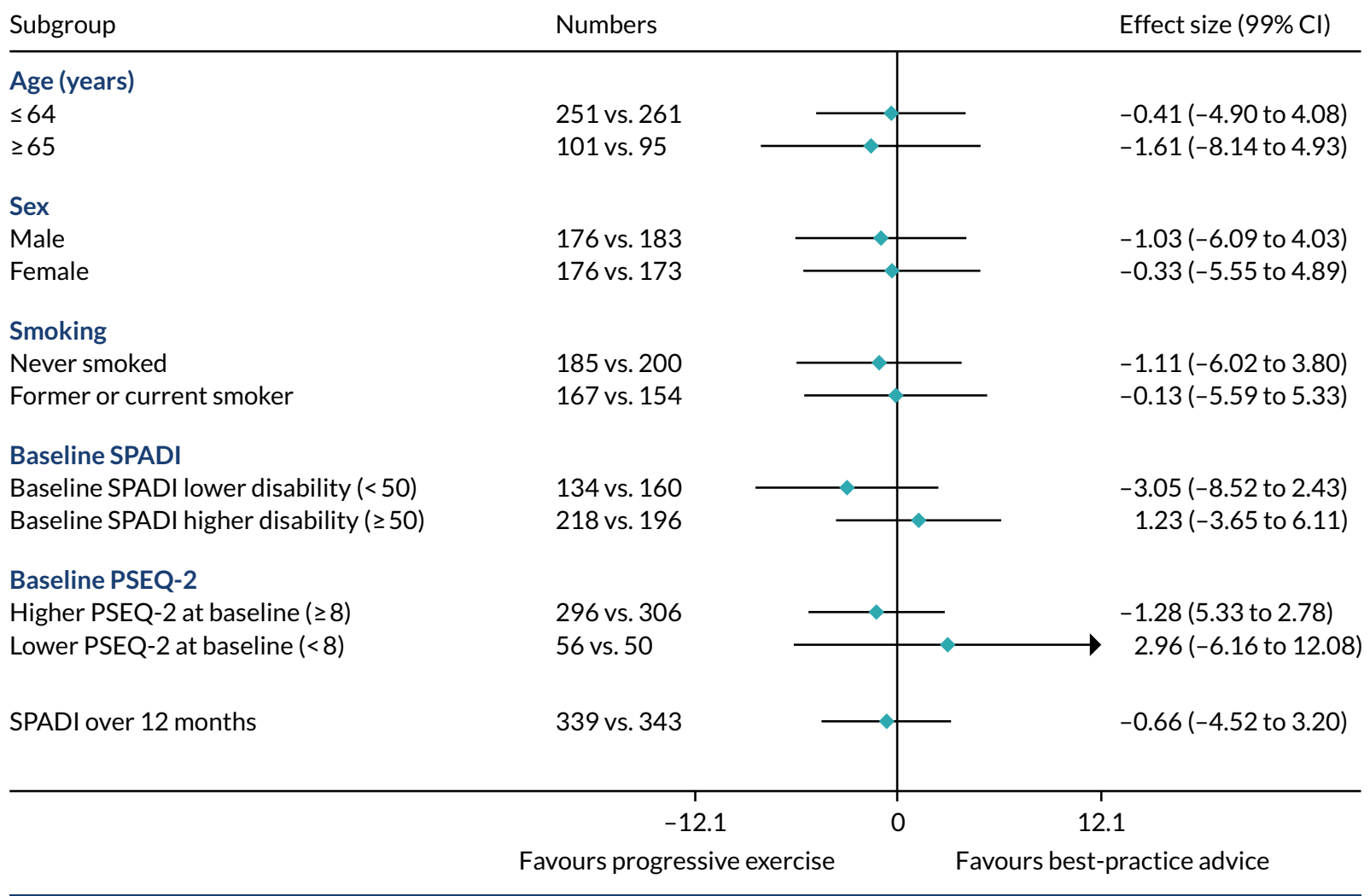

FIGURE 10 Subgroup-adjusted SPADI analysis for progressive exercise over 12 months. 


\section{Injection compared with no injection}

There were no statistically significant subgroup differences for age, sex, smoking status and pain selfefficacy between injection and no injection interventions in shoulder pain and function when analysed over 12 months (Figure 11) or when analysed at 8 weeks, 6 months or 12 months (see Appendix 4, Figure 28). At 8 weeks, the effect of injection was stronger in participants with a higher baseline SPADI score (adjusted MD $-9.67,99 \% \mathrm{Cl}-15.37$ to -3.97 ) than in those who received injection but had a lower baseline SPADI score (adjusted MD $-0.36,99 \% \mathrm{Cl}-6.87$ to 6.16 ). This difference was clinically significant (see Appendix 4, Figure 28). There was no statistically significant subgroup difference when analysed over 12 months or when analysed at 6 and 12 months.

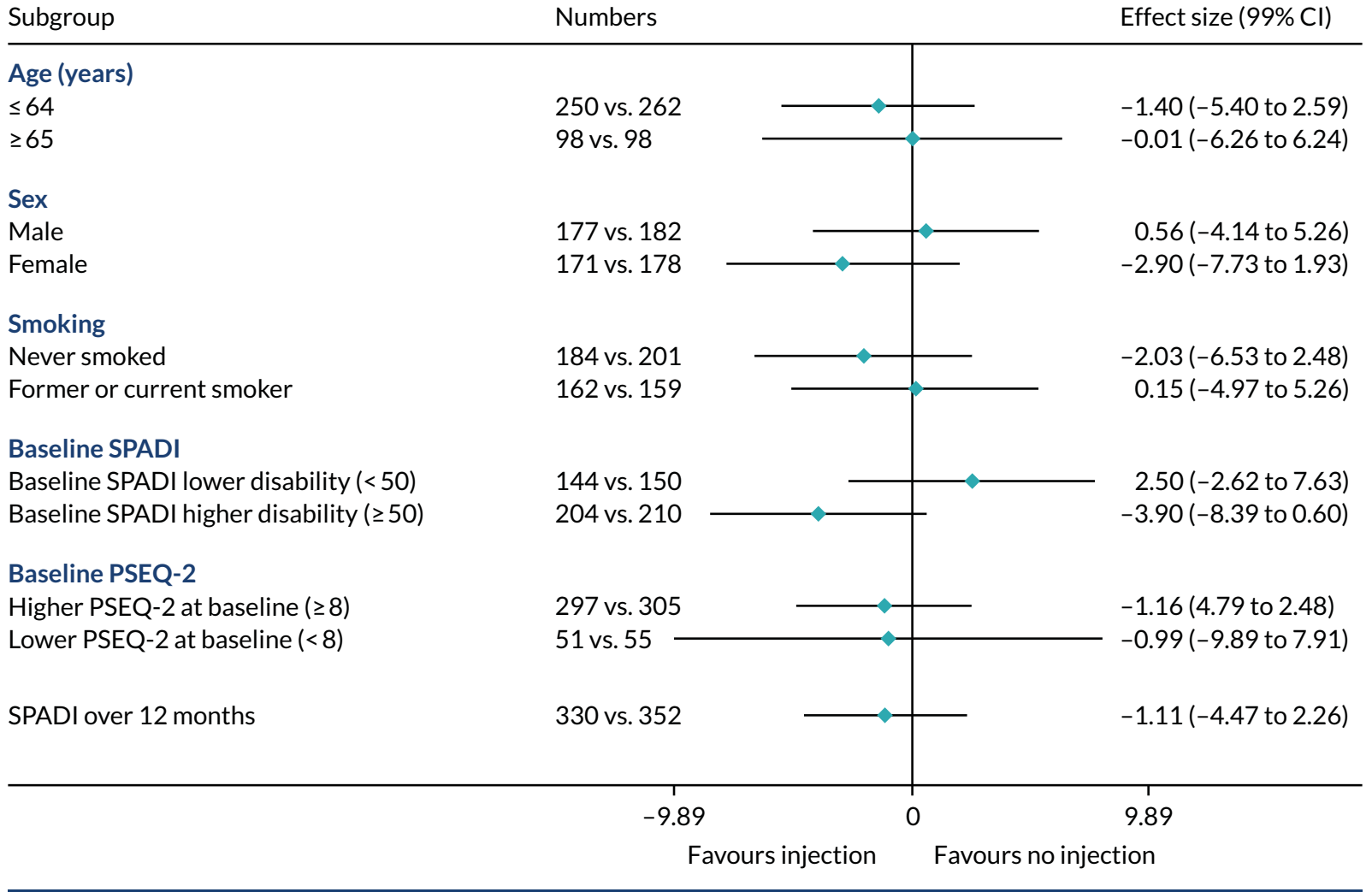

FIGURE 11 Subgroup-adjusted SPADI analysis for injection over 12 months. 


\section{Chapter 5 Health economics}

\section{Introduction}

This chapter presents the cost-effectiveness analysis of the GRASP trial. This analysis took an NHS and PSS perspective and tested (using a $2 \times 2$ factorial design) four physiotherapy-led interventions: (1) progressive exercise, (2) best-practice advice, (3) progressive exercise preceded by a corticosteroid injection and (4) best-practice advice preceded by a corticosteroid injection. Health-care resource utilisation data and utility data were collected alongside clinical data over the 12-month trial period; these were used to conduct a cost-utility analysis, calculating the cost per quality-adjusted life-year (QALY) gained, as the main aim of treatment is to improve patients' quality of life.

Current guidelines for conducting an economic evaluation within clinical trials have been followed, including guidance for resource use data capture, ${ }^{84}$ which highlighted the need for a health economics analysis plan to incorporate time horizon, frequency of data collection and methods of analysis, as well as other important components, such as resource use measure, perspective and unit costs. ${ }^{85} \mathrm{We}$ also followed further guidance on how to conduct economic evaluation alongside randomised trials, 86 and considerations of the methodological issues around these trials. ${ }^{87}$ In relation to conducting an economic evaluation within a factorial design trial, the challenges and methods discussed by Dakin and Gray ${ }^{88}$ have been taken into account in the GRASP trial analysis.

\section{Methods}

Aim

The aim of the GRASP trial's economic evaluation was to address the following question:

What is the cost-effectiveness of individually tailored progressive exercise compared with best-practice advice, with or without corticosteroid injection, in people with a new episode of a rotator cuff disorder?

The within-trial economic analysis was performed using individual patient-level data from the GRASP trial. The analysis uses data from the GRASP trial only and did not combine this trial with any external evidence because, to the best of our knowledge, no study has evaluated exactly the same progressiveexercise intervention evaluated in this study. The analytical approach took the form of a cost-utility analysis. Based on trial evidence, net monetary benefit (NMB) statistics were calculated as QALYs multiplied by willingness-to-pay threshold minus cost to enable comparison between treatment groups.

The economic analysis compared the costs and consequences of each intervention group over the 12-month period following randomisation, with no extrapolation beyond the study period of 12 months, as prespecified in the health economics analysis plan, because there was no statistical difference in clinical outcome between treatment groups at 12 months. ${ }^{85}$

\section{Measurement of resource use and costs}

Resource use data for the economic evaluation were collected during the trial period from questionnaires sent to participants (at 8 weeks, 6 months and 12 months after randomisation to the GRASP trial) and from treatment logs completed by the treating physiotherapists at sites. The questionnaire captured both NHS- and PSS-perspective resource use and costs borne by the participant and their family attributable to a rotator cuff disorder. This included the frequency of use of inpatient care, outpatient care and community-based health care (both private and NHS) that was not part of the GRASP trial. It also recorded direct medical costs that were not part of the trial (e.g. medications and steroid injections) 
and direct non-medical costs (e.g. help with housework/childcare and travel), the latter being excluded from the base-case economic evaluation. Free-text responses (applicable to all the 'other' options) were reclassified to the appropriate cost category, were removed if deemed unrelated/irrelevant to the trial by clinical experts (e.g. shoulder specialists) or were analysed collectively as 'other' in the descriptive analysis.

Some of the assumptions made when cleaning, analysing or costing the data included (1) if a patient answered ' $n o$ ' to a prompt question about resource utilisation, then we assumed that the frequency of service use for this category of resources was equal to zero, (2) injections performed outside the GRASP trial were assumed to have been given by a GP within the duration of a typical GP visit and (3) for the self-reported questions on prescribed and over-the-counter medication, when participants failed to specify the duration or reported 'as needed', 'when in pain' or 'occasionally' and similar, we assumed an intake duration of 3 weeks based on clinical expert opinion.

\section{Costing of the interventions}

\section{Subacromial corticosteroid injection}

Participants randomised to receive corticosteroid injection were given either methylprednisolone or triamcinolone acetonide (median dose of $40 \mathrm{mg}$ ) with a local anaesthetic. Injections were mainly given by extended-scope physiotherapists (bands 6-8a); in three cases injections were given by doctors (two orthopaedic consultants and one specialist registrar in orthopaedics). The consultation time varied from 20 minutes to 45 minutes based on the information provided by the physiotherapists. We based the cost of injections on the median injection administration time of 30 minutes, as a median is more robust against outliers and the weighted average cost per hour for each physiotherapist/clinician delivering injections. ${ }^{89}$ Most participants also received local anaesthetic: either $1 \%$ lidocaine (up to $5 \mathrm{ml}$ ) or $0.5 \%$ bupivacaine hydrochloride (up to $10 \mathrm{ml}$ ) (see Appendix 5, Table 43 for unit costs of corticosteroid injection and anaesthetic). The total cost of administering the injection to each participant was calculated by adding the weighted mean administration cost per participant to the mean cost of corticosteroid injections and mean cost of anaesthetic per participant.

\section{Best-practice advice}

Participants randomised to best-practice advice received one 45- to 60-minute session with a physiotherapist (band 5-8a), when they were provided with a set of eight 2-week exercise diaries (i.e. a normal A4 printed sheet totalling 16 pages), a three-page document printed on non-carbon copy paper that served as an action planner, an information booklet, one piece of resistance band (with an average of 1 yard per participant) and either a DVD or details of how to access online exercise videos (Table 17). The cost of physiotherapists' time was calculated by multiplying the median therapist cost per hour by the median estimate (in minutes) of the exercise session.

\section{Progressive exercise}

Participants randomised to progressive exercise received a median of four sessions with a physiotherapist (band 5-8a). The first session lasted between 45 and 60 minutes and the rest generally lasted between 20 and 30 minutes. Participants were given a set of eight 2-week exercise diaries (i.e. a normal A4 printed sheet totalling 16 pages), a three-page document printed on non-carbon copy paper that served as an action planner, an information booklet and at least one piece of resistance band if they were prescribed an exercise that required this (an average of 1 yard per participant) (see Table 17). Again, the cost of physiotherapists' time was calculated by multiplying the median therapist cost per hour by the median estimate (in minutes) of the exercise session.

In both the best-practice advice and progressive-exercise interventions, we estimated costs based on median estimates, as they are more robust against outliers. Storage boxes given to study centres were excluded from the costings, as they were deemed to be protocol-driven resources. 
TABLE 17 Unit cost of consumables associated with trial per participant

\begin{tabular}{|c|c|c|c|c|c|c|}
\hline Resource & $\begin{array}{l}\text { Best- } \\
\text { practice } \\
\text { advice }\end{array}$ & $\begin{array}{l}\text { Injection plus } \\
\text { best-practice } \\
\text { advice }\end{array}$ & $\begin{array}{l}\text { Progressive } \\
\text { exercise }\end{array}$ & $\begin{array}{l}\text { Injection plus } \\
\text { progressive } \\
\text { exercise }\end{array}$ & $\begin{array}{l}\text { Unit } \\
\text { type }\end{array}$ & $\begin{array}{l}\text { Unit cost } \\
\text { (f) }\end{array}$ \\
\hline Exercise diary & $\checkmark$ & $\checkmark$ & $\checkmark$ & $\checkmark$ & Item/set & 0.64 \\
\hline Action planner & $\checkmark$ & $\checkmark$ & $\checkmark$ & $\checkmark$ & Item & 0.24 \\
\hline $\begin{array}{l}\text { TheraBand }{ }^{\circledR} 1 \text { yard } \\
\text { (TheraBand, Akron, OH, USA) }\end{array}$ & $\checkmark$ & $\checkmark$ & $\checkmark$ & $\checkmark$ & Item & 0.94 \\
\hline $\begin{array}{l}\text { DVD or online access to } \\
\text { exercise videos }\end{array}$ & $\checkmark$ & $\checkmark$ & $x$ & $x$ & Item & 1.20 \\
\hline $\begin{array}{l}\text { Information booklet for } \\
\text { best-practice advice }\end{array}$ & $\checkmark$ & $\checkmark$ & $\checkmark$ & $\checkmark$ & Item & 2.60 \\
\hline $\begin{array}{l}\text { Information booklet for } \\
\text { progressive exercise }\end{array}$ & $x$ & $x$ & $\checkmark$ & $\checkmark$ & Item & 4.00 \\
\hline
\end{tabular}

\section{Training}

We included the cost of training the physiotherapists in how to deliver the best-practice advice and progressive-exercise interventions, as they are not part of standard NHS practice. No training was provided for the injections, as these were delivered as per standard NHS practice and in accordance with the trial protocol.

The cost of training was calculated separately for best-practice advice and progressive exercise by multiplying the mean training time per physiotherapist by the physiotherapist cost per hour (Table 18).

TABLE 18 Assumptions for costing physiotherapist time

\section{Physiotherapist time}

Unit Source

Training for best-practice advice and progressive exercise

Number of physiotherapists

Mean training time (hours) per best-practice advice physiotherapist

Mean training time (hours) per progressive-exercise physiotherapist

Physiotherapist cost $(£)$ per hour

Mean cost $(£)$ of training each best-practice advice physiotherapist

Mean cost $(£)$ of training each progressive-exercise physiotherapist

Mean number of patients treated per best-practice advice physiotherapist

Mean number of patients treated per progressive-exercise physiotherapist

Length of session

First session (minutes)

Second session (minutes)

Third session (minutes)

Fourth session (minutes)

Fifth session (minutes)

Sixth session (minutes) $187^{\mathrm{a}} \quad$ Therapist diaries

3.5 Therapist diaries

4.5

35-67 PSSRU p. 143 (band 5-8a) ${ }^{89}$

168.49

177.94

3.9 Therapist diaries

3.6

25

25

25

PSSRU, Personal Social Service Research Unit.

a Although there 307 clinicians were recorded for the training, some undertook the training twice, which brings the number of therapists down to 298 . However, only 187 physiotherapists both were trained and delivered the exercises. 
Training time for therapists to provide the best-practice advice was 3.5 hours and progressive exercise training was 4.5 hours. In the training courses provided as part of the GRASP trial, physiotherapists received an additional hour of training specific to the trial protocol (e.g. completing treatment logs), which was considered to be a protocol-driven resource use and was, therefore, excluded from the analysis. Some sites received refresher training sessions, with each lasting approximately 2.5 hours.

The face-to-face training was delivered by four physiotherapists who were part of the GRASP trial team, but only one physiotherapist attended each training session. We costed the physiotherapist time for delivering the training by multiplying the cost of a grade 7 physiotherapist by the duration of the training session. In total, 298 physiotherapists (bands 5-8a) attended the training programme as part of the trial, of whom 223 delivered the intervention. The cost of the trainers' time was divided by the total number of physiotherapists attending training.

As there is uncertainty about how training would be delivered in routine clinical practice, we have calculated the cost of training physiotherapists to the interventions based on three scenarios (base case, best case and worst case), depending on different hypotheses.

\section{Base-case analysis}

The base-case analysis aimed to reflect the NHS cost of face-to-face training as it was delivered in the GRASP trial. It included the time cost for the physiotherapists attending the face-to-face training and the time cost for the physiotherapist delivering the training to the sites (Table 19). The cost of refresher training and the trainers' travel costs to each site, venue hire and NHS parking charges were excluded from the analysis. In the base-case analysis, we calculated the total cost of training each physiotherapist

TABLE 19 Cost $(£)$ of intervention per participant

\begin{tabular}{|c|c|c|c|c|}
\hline Cost & $\begin{array}{l}\text { Best-practice } \\
\text { advice (⿷) }\end{array}$ & $\begin{array}{l}\text { Injection plus best- } \\
\text { practice advice (E) }\end{array}$ & $\begin{array}{l}\text { Progressive } \\
\text { exercise (⿷) }\end{array}$ & $\begin{array}{l}\text { Injection plus } \\
\text { progressive exercise (£) }\end{array}$ \\
\hline Clinician training & 43.20 & 43.20 & 49.43 & 49.43 \\
\hline Consumables & 5.62 & 5.62 & 5.82 & 5.82 \\
\hline Injections & 0 & 40.1 & 0 & 40.1 \\
\hline \multicolumn{5}{|c|}{ Physiotherapist time } \\
\hline Session 1 & 44.63 & 44.63 & 44.63 & 44.63 \\
\hline Session 2 & & & 21.25 & 21.25 \\
\hline Session 3 & & & 21.25 & 21.25 \\
\hline Session 4 & & & 21.25 & 21.25 \\
\hline Session 5 & & & 21.25 & 21.25 \\
\hline Session 6 & & & 21.25 & 21.25 \\
\hline \multicolumn{5}{|c|}{ Total cost by number of sessions attended } \\
\hline No sessions & 0 & 0 & 0 & 0 \\
\hline One session & 93.45 & 133.55 & 99.88 & 139.98 \\
\hline Two sessions & & & 121.13 & 161.23 \\
\hline Three sessions & & & 142.38 & 182.48 \\
\hline Four sessions & & & 163.63 & 203.73 \\
\hline Five sessions & & & 184.88 & 224.98 \\
\hline Six sessions & & & 206.13 & 246.23 \\
\hline
\end{tabular}


and divided it by the mean number of patients treated per physiotherapist (among physiotherapists receiving this training) to estimate the cost of training as it was delivered in the trial. The total cost of training each physiotherapist was divided by the mean number of participants treated by each physiotherapist in the trial. We estimated the mean number of participants per physiotherapist separately for best-practice advice and progressive exercise, as physiotherapists giving the best-practice advice intervention could treat more people per week than those delivering progressive exercise. The mean numbers of participants treated per physiotherapist in the best-practice advice and the progressive-exercise groups were 3.9 and 3.6 , respectively.

\section{Worst-case scenario}

This was the same as the base-case analysis, but also included the cost of refresher training, travel, venue hire and NHS parking charges as part of the training delivery cost. Refresher session cost was calculated by multiplying the 2.5 -hour training time by the hourly cost of a grade 7 physiotherapist. Travel cost was calculated as the mileage from Oxford to the site and back by car multiplied by a flat cost per mile ( $£ 0.45$ for the first 10,000 business miles). ${ }^{90}$ Venue hire was calculated based on an average cost of hiring an NHS room of a maximum capacity of 30 people. ${ }^{91} \mathrm{We}$ also assumed an average of $£ 2$ per hour NHS parking charge for the duration of the training. Accommodation charges for overnight stay were not included.

\section{Best-case scenario}

The best-case scenario assumes that if the trial interventions were to be implemented in routine clinical practice, the training would be delivered on a free-to-access online platform. The cost of developing the online training materials were based on the team's experience of developing training materials for the NIHR HTA-funded BeST (Back Skills Training) trial for the treatment of low back pain in primary care. ${ }^{45}$ This includes 6 weeks of a full-time grade 8 physiotherapist's time to develop the training materials for the progressive-exercise intervention and 1 month to develop the best-practice advice intervention. It also includes the cost of a grade 7 physiotherapist researcher to maintain it for a 10-year period (spending 30 hours/year). There are approximately 10,000 users per year using the online learning platform [URL: www.futurelearn.com/courses/back-skills-training-programme (accessed 24 May 2021)]. We, therefore, assumed that this researcher would support all 10,000 learners. We included the cost of the physiotherapists treating participants based on having a one-off training session of 3.5 hours for best-practice advice and of 4.5 hours for progressive exercise. We assumed that the training would last 10 years without a refresher session and that each physiotherapist would treat patients for 10 years after being trained. We assumed that each physiotherapist would treat approximately 100 patients per year, based on an assumption of 2.74 new patients per week over 1 year (9.6 patients per week and 3.5 patients per session on average).

The best- and worst-case scenarios have been presented as part of the sensitivity analysis.

We averaged and applied the cost of training for each intervention across all participants randomised, regardless of how many sessions they attended. Other intervention costs were estimated at the individual patient level, based on the recorded number of sessions that they attended. The estimated costs for different numbers of sessions attended of progressive exercise, best-practice advice, progressive exercise preceded by a corticosteroid injection and best-practice advice preceded by a corticosteroid injection are shown in Table 19. Following our base-case assumptions, the cost of best-practice advice is $£ 93.45$ and best-practice advice plus injection is $£ 133.55$. For a progressive-exercise patient attending all six sessions, the cost is $£ 206.13$, compared with $£ 246.23$ for a patient attending all recommended sessions in the progressive-exercise and injection treatment arm.

\section{Measurement of broader resource use}

The unit costs of direct non-medical resource items, such as help with child care, travel to appointments, help with housework and any other additional expenses attributable to having a rotator cuff disorder 
incurred by the participant, were obtained directly from the postal follow-up questionnaires and are tabulated in Appendix 5, Table 44. However, these costs, private health-care costs and any other non-NHS/PSS costs (e.g. over-the-counter medications) were excluded from the base-case economic evaluation, as they are outside the perspective of this analysis, but are included in the sensitivity analysis presented in Appendix 5.

\section{Valuation of resource use}

Unit costs of direct medical care that is not part of the trial, such as inpatient care, outpatient care and NHS community care, were sourced from the latest available NHS Reference Costs ${ }^{92}$ (see Appendix 5, Table 44). The unit costs of medications related to rotator cuff disorders have been sourced using the latest available British National Formulary (BNF)93 (see Appendix 5, Table 43). Costs of medications for individual participants were estimated based on their reported doses and frequencies, when these were available, or based on an assumed daily dose using BNF recommendations. When a dose range was reported as 'as required' or when the quantities were not recorded, expert opinion was sought to make reasonable assumptions. The cost of NHS health-care resource use per participant was computed by multiplying the frequency of health resource utilisation reported by the participant by the unit cost of each resource item. In the case of non-NHS costs, participants self-reported the cost of additional expenses. All costs were expressed in 2018/19 Great British pounds. No discounting was applied, as the time horizon of the analysis did not exceed 12 months.

\section{Calculation of utilities and quality-adjusted life-years}

Participants' questionnaires contained the EQ-5D-5L questionnaire for self-completion at baseline and at 8 weeks, 6 months and 12 months post randomisation. The EQ-5D-5L instrument ${ }^{94}$ facilitates the generation of a utility score from a person's health-related quality of life while reducing the ceiling effect and being more sensitive than its three-level predecessor. ${ }^{95} \mathrm{~A}$ utility score refers to the preference of the general population for any particular set of health outcomes. As per the NICE position statement, the responses to the EQ-5D-5L were converted into multiattribute utility scores using the approved 'crosswalk' to the three-level instrument and applying the mapping function developed by van Hout et al., ${ }^{96}$ and the converted responses were valued using the established time trade-off utility algorithm for the UK. ${ }^{97}$ QALYs were calculated as the area under the utility curve of utility scores from baseline, 8-week, 6-month and 12-month data using the trapezoidal rule. ${ }^{98}$ Deceased patients were assigned a utility of zero from the date of death. We assumed that utility remained constant between the last utility measurement and the date of death.

\section{Missing data}

Because within-trial health economic evaluations draw on many sources of information on patient characteristics, treatments, outcomes and resource use over the whole trial, incomplete data are a particular issue that require careful attention. Consequently, the base-case analysis imputed missing data using fully conditional multiple imputation under chained equations, using the Stata command 'mi impute chained'. Within multiple imputation under chained equations, regression models were used to impute unobserved costs and utilities at each time point using the baseline covariates [i.e. age, sex and EuroQol-5 Dimensions (EQ-5D)] as predictor variables. The imputation model included a dummy variable for allocation to injection, a dummy variable for allocation to progressive exercise and an interaction term equal to the product of these two variables, following recommended practice for factorial trials. ${ }^{88}$ Different components of costs and EQ-5D utility scores at each time point contributed as both predictors and imputed variables.

Multiple imputation was used to generate 25 data sets (or 'draws') using predictive mean matching, which provides plausible values when costs and utilities are non-normally distributed. In line with recommended practice, ${ }^{99}$ the imputation model was validated by comparing the distributions of the imputed data with the observed data. The imputation was run following the rule of thumb that the number of imputations $(M)$ should be similar to the percentage of incomplete cases (in this case $M=25$ ). ${ }^{99}$ 


\section{Analyses of resource use, costs and outcome data}

\section{Cost-effectiveness analysis}

The analysis was conducted based on the ITT principle and incremental cost-effectiveness ratios (ICERs) were calculated as the difference in mean costs divided by the difference in mean QALYs between a pair of interventions. The NICE ${ }^{100}$ cost-effectiveness threshold of $£ 20,000$ per additional QALY was used to identify which of the following treatments represent best value for money (i.e. has the highest NMB): (1) best-practice advice, (2) best-practice advice plus corticosteroid injection, (3) progressive exercise or (4) progressive exercise plus corticosteroid injection.

In addition to calculating and reporting ICERs and NMBs, the results are presented graphically in cost-effectiveness planes, and cost-effectiveness acceptability curves (CEACs) are used to show the probability that each of the four treatment groups has the highest NMB. Measures of uncertainty (SEs and Cls) are also reported around the mean costs and QALYs (95\% Cls are presented around ICERs if they are defined). SEs and CEACs were generated using non-parametric bootstrapping with 1000 replicates, as described in the next section. This accommodates sampling (or stochastic) uncertainty and varying levels of willingness to pay for an additional QALY. We made no adjustment for clustering of participants by physiotherapist when analysing costs, QALYs or cost-effectiveness, as the randomisation was carried out on an individual basis, stratified by centre, rather than using cluster randomisation, and the subacromial corticosteroid injections and physiotherapy sessions were delivered in accordance with a standard protocol.

As the GRASP trial is a factorial trial, it was important to consider the interactions [i.e. to examine whether or not the differences in costs, QALYs or NMB between best-practice advice and progressive exercise were affected by the use of corticosteroid injection (or vice versa)]. In the GRASP trial clinical analysis, the primary outcome was analysed at the margins, assuming no interactions and that interaction terms were included in the model only if interactions were significant at the 0.05 level. From the economics point of view, this approach was not appropriate, as health economics findings are interpreted in terms of the absolute magnitude of ICERs, rather than focusing on hypothesis testing, and several mechanisms have been suggested that may introduce large but non-significant interactions for economic end point, but not clinical end point. ${ }^{88} \mathrm{It}$ is more important to avoid the bias that may result from ignoring interactions rather than maximising statistical power. We, therefore, compared specific treatment combinations (e.g. best-practice advice, injection only, progressive exercise only and injection plus progressive exercise) incrementally and identified the combination that represents best value for money, rather than making separate decisions on injection and exercise, as the former provides more relevant information for decision-makers if there is any interaction.

For the GRASP trial, the base-case economic analysis was prespecified as regression analysis with an interaction term, although regression analysis without interaction terms has been used as a sensitivity analysis to assess whether or not the assumptions about interactions change the conclusions of the analysis. Benefits of using regression analysis in the context of factorial design trial are that it allows for variation in sample size between groups, adjusts for the effect of the other intervention, facilitates adjustment for baseline utility and can predict the mean outcomes for each cell in the factorial design.

\section{Regression analysis with interaction term (base-case analysis)}

Linear regression analyses that predicted both costs and QALYs were calculated for each bootstrap sample on each imputed data set. Randomisation to corticosteroid and randomisation to exercise were included as dummy variables and the base-case analysis also included an interaction between these two variables. The ordinary least squares regression that predicted QALYs also controlled for baseline utility to avoid the bias that would otherwise arise from any imbalance in baseline utility between groups. ${ }^{101} \mathrm{~A}$ total of 1000 bootstrap samples were drawn for each imputed data set. Mean costs, QALYs and the NMB in each of the four treatment groups were estimated based on the regression coefficients for each bootstrap of each imputed data set. 
We combined uncertainty around missing data with sampling uncertainty using the MI Boot pooled sample approach of Schomaker and Heumann, ${ }^{102}$ which has been shown to yield valid inference and to be equivalent to nesting bootstraps within imputations and combining results using Rubin's rule. Pooling bootstraps is simpler to implement than Rubin's rule and facilitates presentation of CEACs. In addition, a simulation study has shown it to give good coverage with $\geq 20$ imputations. To implement this in our data set, we averaged across the 25 imputed data sets for the original (non-bootstrapped) sample to get point estimates and estimated $95 \% \mathrm{Cls}$ as the 2.5 th and 97.5 th percentiles across all 25,000 bootstraps, adapting the code used previously. ${ }^{103}$ CEACs were estimated across all 25,000 bootstraps. The regression models used to predict cost and QALYs are presented in Appendix 5, Model 1: regression analysis model with interaction term (base-case analysis).

\section{Regression analysis without interaction term (sensitivity analysis)}

Regression techniques with an interaction term provide an alternative to at-the-margins analysis, which also assumes no interaction. ${ }^{88}$ The description of the analysis and the model are presented in Appendix 5, Model 2: regression analysis without interaction term (sensitivity analysis).

\section{Results of economic analysis}

\section{Completion rate}

Among the 708 participants randomised in the trial, 174 were randomised to best-practice advice, 178 were randomised to best-practice advice plus injection, 174 were randomised to progressive exercise and 182 were randomised to progressive exercise plus injection. The completion rates of all health resource items by treatment intervention for each time point are displayed in Table 20. In addition, Table 21 shows the response rate of EQ-5D-5L by follow-up points and treatment group.

\section{Health-care resource use and costs}

Information about the use of other relevant NHS services was obtained by participant self-reported data at 8 weeks, 6 months and 12 months. Estimates of health-care use have been presented in Appendix 5 as follows: from baseline to 8 weeks (see Appendix 5, Table 45), from 8 weeks to 6 months (see Appendix 5 , Table 46) and from 6 to 12 months (see Appendix 5, Table 47). These resource quantities were multiplied by the relevant unit cost (see Appendix 5, Table 44) to provide estimated mean costs per patient from baseline to 8 weeks (see Appendix 5, Table 48), from 8 weeks to 6 months (see Appendix 5, Table 49) and from 6 to 12 months (see Appendix 5, Table 50).

Prescribed medication usage was recorded at 8 weeks, 6 months and 12 months. Participants were asked to list the drugs that they were currently taking and to report the dose and frequency of use. The yielded estimate of the cost of prescribed medications for each individual over the three time intervals can be seen in Appendix 5, Table 51. The mean cost per participant of prescribed medication over the 12-month period was quite low for all treatment groups ( $£ 10.89$ for best-practice advice compared with $£ 5.36$ for best-practice advice plus injection, $£ 2.90$ for progressive exercise and $£ 17.57$ for progressive exercise plus injection). A summary of all included costs from the NHS and PSS perspective (i.e. intervention cost, NHS service utilisation cost, prescribed medication cost and cost of non-GRASP trial steroid injection) over the trial are given in Table 22. It is worth noting that NHS service cost is lower in the progressive-exercise group than in the other intervention groups, which indicates that participants in the progressive-exercise intervention group made less use of primary and secondary health-care services.

Table 23 presents the mean cost and SD of non-NHS costs and any additional expenses, medication and private care that was borne by GRASP trial participants. In terms of employment status, at 8 weeks, $49.44 \%$ of participants were in paid employment, of whom $4.66 \%$ took time off work. At 6 and 12 months, the percentages were quite similar, with $46.06 \%$ and $46.75 \%$ of the participants being in paid employment and only $3.81 \%$ and $3.67 \%$, respectively, taking time off work. The mean cost of work loss by treatment 
TABLE 20 Completion rate (\%) of health resource use by treatment interventions and follow-up time points

\begin{tabular}{|c|c|c|c|c|c|c|c|c|c|c|c|c|}
\hline \multirow[b]{2}{*}{ Type of care } & \multicolumn{3}{|c|}{ Best-practice advice $(\mathrm{N}=174)$} & \multicolumn{3}{|c|}{$\begin{array}{l}\text { Injection plus best-practice advice } \\
(N=178)\end{array}$} & \multicolumn{3}{|c|}{ Progressive exercise $(N=174)$} & \multicolumn{3}{|c|}{$\begin{array}{l}\text { Injection plus progressive exercise } \\
(\mathrm{N}=182)\end{array}$} \\
\hline & $\begin{array}{l}\text { Yes, } \\
n(\%)\end{array}$ & $\begin{array}{l}\text { No, } \\
n(\%)\end{array}$ & $\begin{array}{l}\text { Missing, } \\
n(\%)\end{array}$ & $\begin{array}{l}\text { Yes, } \\
n(\%)\end{array}$ & $\begin{array}{l}\text { No, } \\
n(\%)\end{array}$ & $\begin{array}{l}\text { Missing, } \\
n(\%)\end{array}$ & $\begin{array}{l}\text { Yes, } \\
n(\%)\end{array}$ & $\begin{array}{l}\text { No, } \\
n(\%)\end{array}$ & $\begin{array}{l}\text { Missing, } \\
n(\%)\end{array}$ & $\begin{array}{l}\text { Yes, } \\
n(\%)\end{array}$ & $\begin{array}{l}\text { No, } \\
n(\%)\end{array}$ & $\begin{array}{l}\text { Missing, } \\
n(\%)\end{array}$ \\
\hline \multicolumn{13}{|l|}{ Baseline to 8 weeks } \\
\hline $\begin{array}{l}\text { Primary care (NHS } \\
\text { community-based services) }\end{array}$ & $16(9.20)$ & $113(64.94)$ & $45(25.86)$ & $8(4.49)$ & $142(79.78)$ & $28(15.73)$ & $22(12.64)$ & $124(71.26)$ & $28(16.09)$ & $11(6.04)$ & $151(82.97)$ & 20 (10.99) \\
\hline $\begin{array}{l}\text { Secondary care (NHS } \\
\text { outpatient services) }\end{array}$ & $6(3.45)$ & $123(70.69)$ & $45(25.86)$ & $6(3.37)$ & $144(80.90)$ & $28(15.73)$ & $10(5.75)$ & $136(78.16)$ & $28(16.09)$ & $3(1.65)$ & $160(87.91)$ & $19(10.44)$ \\
\hline Private care & $2(1.15)$ & $127(72.99)$ & $45(25.86)$ & $1(0.56)$ & $150(84.27)$ & $27(15.17)$ & $3(1.72)$ & $143(82.18)$ & $28(16.09)$ & 0 & $162(89.01)$ & 20 (10.99) \\
\hline Injection utilisation & $1(0.57)$ & $129(74.14)$ & $44(25.29)$ & 7 (3.93) & $144(80.90)$ & $27(15.17)$ & $3(1.72)$ & $144(82.76)$ & $27(15.52)$ & $10(5.49)$ & $153(84.07)$ & $19(10.44)$ \\
\hline Non-medical expenses & $5(2.87)$ & $124(71.26)$ & $45(25.86)$ & $5(2.81)$ & $145(81.46)$ & $28(15.73)$ & 15 (8.62) & $131(75.29)$ & $28(16.09)$ & $18(9.89)$ & $144(79.12)$ & 20 (10.99) \\
\hline \multicolumn{13}{|l|}{8 weeks to 6 months } \\
\hline $\begin{array}{l}\text { Primary care (NHS } \\
\text { community-based services) }\end{array}$ & $16(9.20)$ & 111 (63.79) & 47 (27.01) & $15(8.43)$ & $132(74.16)$ & $31(17.42)$ & $18(10.34)$ & $122(70.11)$ & $34(19.54)$ & $16(8.79)$ & $134(73.63)$ & 32 (17.58) \\
\hline $\begin{array}{l}\text { Secondary care (NHS } \\
\text { outpatient services) }\end{array}$ & $7(4.02)$ & $120(68.97)$ & 47 (27.01) & $9(5.06)$ & $137(76.97)$ & $32(17.98)$ & $8(4.60)$ & $132(75.86)$ & $34(19.54)$ & $2(1.10)$ & $148(81.32)$ & $32(17.58)$ \\
\hline Private care & $8(4.60)$ & $120(68.97)$ & $46(26.44)$ & $6(3.37)$ & $141(79.21)$ & 31 (17.42) & $8(4.60)$ & $132(75.86)$ & $34(19.54)$ & $4(2.20)$ & $146(80.22)$ & 32 (17.58) \\
\hline Injection utilisation & $9(5.17)$ & $121(69.54)$ & $44(25.29)$ & $6(3.37)$ & $141(79.21)$ & 31 (17.42) & $9(5.17)$ & $131(75.29)$ & $34(19.54)$ & $6(3.30)$ & $143(78.57)$ & 33 (18.13) \\
\hline Non-medical expenses & $8(4.60)$ & $121(69.54)$ & $45(25.86)$ & $6(3.37)$ & $141(79.21)$ & 31 (17.42) & $12(6.90)$ & $128(73.56)$ & $34(19.54)$ & $13(7.14)$ & $137(75.27)$ & 32 (17.58) \\
\hline \multicolumn{13}{|l|}{ 6-12 months } \\
\hline $\begin{array}{l}\text { Primary care (NHS } \\
\text { community-based services) }\end{array}$ & $17(9.77)$ & $116(66.67)$ & $41(23.56)$ & $22(12.36)$ & $128(71.91)$ & $28(15.73)$ & $14(8.05)$ & $126(72.41)$ & $34(19.54)$ & 24 (13.19) & $128(70.33)$ & $30(16.48)$ \\
\hline $\begin{array}{l}\text { Secondary care (NHS } \\
\text { outpatient services) }\end{array}$ & $10(5.75)$ & $123(70.69)$ & $41(23.56)$ & $15(8.43)$ & $135(75.84)$ & $28(15.73)$ & $9(5.17)$ & $132(75.86)$ & $33(18.97)$ & $15(8.24)$ & $137(75.27)$ & $30(16.48)$ \\
\hline Private care & $8(4.60)$ & $125(71.84)$ & $41(23.56)$ & $3(1.69)$ & 147 ( 82.58) & $28(15.73)$ & $8(4.60)$ & $133(76.44)$ & $33(18.97)$ & $9(4.95)$ & $143(78.57)$ & $30(16.48)$ \\
\hline Injection utilisation & $12(6.90)$ & $121(69.54)$ & $41(23.56)$ & 8 (4.49) & 142 (79.78) & $28(15.73)$ & $9(5.17)$ & $132(75.86)$ & $33(18.97)$ & $12(6.59)$ & $138(75.82)$ & 32 (17.58) \\
\hline Non-medical expenses & $8(4.60)$ & $125(71.84)$ & $41(23.56)$ & $8(4.49)$ & 142 (79.78) & $28(15.73)$ & $6(3.45)$ & $135(77.59)$ & $33(18.97)$ & $10(5.49)$ & $142(78.02)$ & 30 (16.48) \\
\hline
\end{tabular}


TABLE 21 Response rate (\%) of EQ-5D-5L by follow-up time point and treatment

\begin{tabular}{|c|c|c|c|c|c|c|c|c|}
\hline \multirow[b]{2}{*}{ Time point } & \multicolumn{2}{|c|}{$\begin{array}{l}\text { Best-practice advice } \\
(\mathrm{N}=174)\end{array}$} & \multicolumn{2}{|c|}{$\begin{array}{l}\text { Injection plus } \\
\text { best-practice } \\
\text { advice }(N=178)\end{array}$} & \multicolumn{2}{|c|}{$\begin{array}{l}\text { Progressive exercise } \\
(\mathrm{N}=174)\end{array}$} & \multicolumn{2}{|c|}{$\begin{array}{l}\text { Injection plus } \\
\text { progressive } \\
\text { exercise }(N=182)\end{array}$} \\
\hline & $n$ & Missing, $\boldsymbol{n}(\%)$ & $n$ & Missing, $\boldsymbol{n}(\%)$ & $n$ & Missing, $\boldsymbol{n}(\%)$ & $n$ & Missing, $\boldsymbol{n}(\%)$ \\
\hline 8 weeks & 132 & $42(24.14)$ & 151 & 27 (15.17) & 147 & $27(15.52)$ & 164 & $18(9.89)$ \\
\hline 6 months & 134 & 40 (22.99) & 151 & $27(15.17)$ & 143 & 31 (17.82) & 154 & $28(15.38)$ \\
\hline 12 months & 138 & $36(20.69)$ & 158 & $20(11.24)$ & 144 & $30(17.24)$ & 154 & $28(15.38)$ \\
\hline
\end{tabular}

TABLE 22 Health-care cost (£) over the 12-month follow-up (available cases, without imputation of missing data)

\begin{tabular}{|c|c|c|c|c|c|c|c|c|}
\hline \multirow[b]{2}{*}{ Type of care } & \multicolumn{2}{|c|}{$\begin{array}{l}\text { Best-practice advice } \\
(N=174)\end{array}$} & \multicolumn{2}{|c|}{$\begin{array}{l}\text { Injection plus } \\
\text { best-practice } \\
\text { advice ( }=178)\end{array}$} & \multicolumn{2}{|c|}{$\begin{array}{l}\text { Progressive exercise } \\
(N=174)\end{array}$} & \multicolumn{2}{|c|}{$\begin{array}{l}\text { Injection plus } \\
\text { progressive exercise } \\
(\mathrm{N}=182)\end{array}$} \\
\hline & $n^{a}$ & $\begin{array}{l}\text { Mean cost (E) } \\
\text { (SD) }\end{array}$ & $n^{\mathrm{a}}$ & $\begin{array}{l}\text { Mean cost (E) } \\
\text { (SD) }\end{array}$ & $n^{a}$ & $\begin{array}{l}\text { Mean cost (E) } \\
\text { (SD) }\end{array}$ & $n^{a}$ & $\begin{array}{l}\text { Mean cost (E) } \\
\text { (SD) }\end{array}$ \\
\hline Intervention & 167 & $43.20(0)$ & 164 & $43.20(0)$ & 166 & $49.43(29.50)$ & 171 & $49.43(30.94)$ \\
\hline NHS services & 174 & 60.44 (311.76) & 178 & 61.29 (312.85) & 174 & 39.33 (103.79) & 182 & 61.19 (317.38) \\
\hline Prescriptions & 123 & 10.89 (64.31) & 145 & 5.36 (18.19) & 138 & 2.90 ( 8.44$)$ & 148 & 17.57 (74.96) \\
\hline $\begin{array}{l}\text { Corticosteroid } \\
\text { injections, not } \\
\text { as part of } \\
\text { GRASP trial }\end{array}$ & 103 & $10.67(28.81)$ & 127 & $8.65(23.55)$ & 120 & $10.46(33.64)$ & 132 & $12.29(34.12)$ \\
\hline $\begin{array}{l}\text { Total cost } \\
\text { (NHS and } \\
\text { PSS) }\end{array}$ & 103 & $193.31(414.85)$ & 119 & $203.23(146.97)$ & 116 & 236.95 (151.67) & 124 & $308.68(407.61)$ \\
\hline 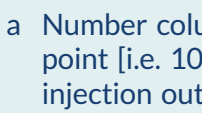 & in & $\begin{array}{l}s \text { to the particip } \\
174 \text { participant } \\
\text { JRASP trial, of }\end{array}$ & S V & $\begin{array}{l}\text { nswered the } \\
\text { t-practice advi } \\
\text { participant }(0 .\end{array}$ & io & $\begin{array}{l}\text { you use } X \text { re } \\
\text { sponded to th } \\
\text { injection (see }\end{array}$ & ce t & $\begin{array}{l}\text { at each time } \\
\text { if they had an }\end{array}$ \\
\hline
\end{tabular}

TABLE 23 Non-NHS costs (£) over the 12 months (available cases, without imputation of missing data)

\begin{tabular}{|c|c|c|c|c|c|c|c|c|}
\hline \multirow[b]{2}{*}{$\begin{array}{l}\text { Non-NHS } \\
\text { cost }\end{array}$} & \multicolumn{2}{|c|}{$\begin{array}{l}\text { Best-practice advice } \\
(N=174)\end{array}$} & \multicolumn{2}{|c|}{$\begin{array}{l}\text { Injection plus } \\
\text { best-practice } \\
\text { advice }(N=178)\end{array}$} & \multicolumn{2}{|c|}{$\begin{array}{l}\text { Progressive exercise } \\
(N=174)\end{array}$} & \multicolumn{2}{|c|}{$\begin{array}{l}\text { Injection plus } \\
\text { progressive exercise } \\
(N=182)\end{array}$} \\
\hline & $n^{a}$ & $\begin{array}{l}\text { Mean cost (E) } \\
\text { (SD) }\end{array}$ & $n^{\mathrm{a}}$ & $\begin{array}{l}\text { Mean cost (E) } \\
\text { (SD) }\end{array}$ & $n^{\mathrm{a}}$ & $\begin{array}{l}\text { Mean cost }(\mathrm{f}) \\
\text { (SD) }\end{array}$ & $n^{a}$ & $\begin{array}{l}\text { Mean cost (£) } \\
\text { (SD) }\end{array}$ \\
\hline Time off work & 174 & $107.58(576.68)$ & 178 & $105.17(658.46)$ & 174 & 36.98 (309.12) & 182 & $102.86(704.33)$ \\
\hline $\begin{array}{l}\text { Additional } \\
\text { expenses }\end{array}$ & 109 & 36.99 (189.04) & 134 & $13.62(94.41)$ & 131 & 33.58 (209.86) & 143 & $280.49(3202.68)$ \\
\hline $\begin{array}{l}\text { Medication } \\
\text { (out of } \\
\text { pocket) }\end{array}$ & 115 & $13.34(30.97)$ & 132 & $15.71(29.64)$ & 127 & 20.11 (49.82) & 139 & $17.74(38.90)$ \\
\hline Private care & 174 & $22.07(131.22)$ & 178 & $16.98(161.47)$ & 174 & $15.95(79.78)$ & 182 & $18.62(100.24)$ \\
\hline $\begin{array}{l}\text { Total cost } \\
\text { (societal) }\end{array}$ & 101 & 229.78 (926.49) & 123 & 185.68 (818.29) & 119 & $110.30(412.65)$ & 133 & 441.39 (3448.15) \\
\hline
\end{tabular}

a The number column refers to the participants who answered the question 'did you use $\mathrm{X}$ resource use?' at each time point. 
allocation for the 12-month period is also shown in Table 23. Table 23 further indicates that the non-NHS cost of 'additional expenses' and 'total cost (societal)' was higher in the injection and progressive-exercise intervention groups than in the other intervention groups. Utilisation of corticosteroid injections outside the GRASP trial is presented in Appendix 5, Table 52. The use of corticosteroid injections outside the trial varied slightly across treatment groups and follow-up periods, but, overall, was very low, with the largest number of participants receiving injection $(n=12)$ recorded between 6 and 12 months from the bestpractice advice group. In addition, $<1 \%$ of participants reported paying for an injection privately. Similarly, physiotherapy sessions taken outside the GRASP trial have been recorded and presented in Appendix 5, Tables 44-47. The number of physiotherapy sessions taken outside the trial was, overall, very small across all treatment groups and follow-up periods, and varied slightly.

\section{Utility and quality-adjusted life-years}

Utility scores were estimated using validated EQ-5D-5L questionnaires completed by participants at baseline, 8 weeks, 6 months and 12 months. The summary statistics of the unadjusted and adjusted EQ-5D utility scores for all observed cases across all time points by treatment interventions are presented in Table 24 and Appendix 5, Table 53. EQ-5D-5L scores at 12 months were higher than the baseline scores in all treatment groups. Baseline EQ-5D was markedly higher in the group randomised to progressive exercise only. Consequently, unadjusted EQ-5D utilities and QALYs should be interpreted with caution.

TABLE 24 Utility and QALY estimates: EQ-5D-5L scores (available cases with and without imputation and adjustment for baseline utility)

\begin{tabular}{llllllll} 
Utility and QALY & $\begin{array}{l}\text { Best-practice } \\
\text { advice }\end{array}$ & $\begin{array}{l}\text { Injection plus } \\
\text { best-practice advice } \\
\text { estimate }\end{array}$ & $\begin{array}{llllll}\text { Progressive } \\
\text { exercise }\end{array}$ & $\begin{array}{l}\text { Injection plus } \\
\text { progressive exercise }\end{array}$ \\
\hline
\end{tabular}

Available cases without imputation and no adjustment for baseline utility

$\begin{array}{lllllllll}\text { Baseline } & 174 & 0.65(0.20) & 178 & 0.64(0.18) & 172 & 0.69(0.15) & 181 & 0.64(0.18) \\ 8 \text { weeks } & 132 & 0.68(0.20) & 151 & 0.73(0.17) & 147 & 0.70(0.15) & 164 & 0.69(0.20) \\ 6 \text { months } & 134 & 0.74(0.20) & 151 & 0.75(0.16) & 143 & 0.78(0.16) & 154 & 0.72(0.23) \\ 12 \text { months } & 138 & 0.77(0.20) & 158 & 0.77(0.16) & 144 & 0.81(0.16) & 154 & 0.75(0.22) \\ \text { QALYs } & 101 & 0.73(0.17) & 132 & 0.75(0.12) & 124 & 0.76(0.28) & 137 & 0.73(0.18)\end{array}$

Imputation and no adjustment for baseline utility

$\begin{array}{lllllllll}\text { Baseline } & 174 & 0.65(0.20) & 178 & 0.64(0.18) & 174 & 0.69(0.15) & 182 & 0.64(0.18) \\ 8 \text { weeks } & 174 & 0.69(0.20) & 178 & 0.72(0.17) & 174 & 0.69(0.15) & 182 & 0.68(0.21) \\ 6 \text { months } & 174 & 0.73(0.20) & 178 & 0.75(0.17) & 174 & 0.76(0.17) & 182 & 0.72(0.23) \\ 12 \text { months } & 174 & 0.76(0.21) & 178 & 0.77(0.16) & 174 & 0.81(0.16) & 182 & 0.76(0.21) \\ \text { QALYs } & 174 & 0.72(0.18) & 178 & 0.75(0.13) & 174 & 0.77(0.28) & 182 & 0.74(0.17)\end{array}$

Imputation and adjustment for baseline utility

QALYs

$174 \quad 0.74(0.15) \quad 178$

$0.74(0.15)$

$174 \quad 0.77(0.12) \quad 182$

$0.72(0.17)$

a The number column refers to the participants who answered the question 'did you use $\mathrm{X}$ resource use?' at each time point. 


\section{Cost-effectiveness results}

\section{Base-case results}

Table 25 presents the costs and QALYs associated with the four interventions under investigation. This analysis evaluated the impact of exercise treatment and injection and interactions between these two interventions inside the table, while imputing missing values and adjusting for age, sex and baseline utility.

When all randomised patients were included in the analysis and missing values were imputed using multiple imputation, patients receiving best-practice advice accrued an average of $0.737(95 \% \mathrm{Cl} 0.710$ to 0.763 ) QALYs and an NHS cost of $£ 195$ over the 12-month period (see Table 25 ). In the base-case analysis, adding injection to best-practice advice gained 0.021 QALYs $(p=0.184)$ and increased the cost by $£ 10$ per participant $(p=0.747)$ compared with best-practice advice alone. Progressive exercise alone was $£ 52(p=0.247)$ more expensive per participant than best-practice advice, while gaining 0.019 QALYs $(p=0.220)$. However, there was a non-significant interaction for cost $(p=0.397)$, which meant that when injection was added to progressive exercise it generated an additional cost of $£ 60$ per participant (i.e. $£ 50$ more than the difference between best-practice advice and injection only). There was also a non-significant qualitative interaction for QALYs $(p=0.106)$, whereby adding injection to best-practice advice increased QALYs, but adding injection to progressive exercise reduced QALYs; the groups receiving injection alone or progressive exercise alone accrued more QALYs than the group receiving best-practice advice, whereas the group that received both of these treatments had lower QALYs than either of the groups receiving only one treatment. Best-practice advice plus injection cost $£ 475.59$ more per QALY gained than best-practice advice alone. Progressive exercise alone and progressive exercise plus injection were both strongly dominated by best-practice advice plus injection, being both more costly and accruing fewer QALYs.

The 2013 NICE cost-effectiveness threshold of $£ 20,000$ per additional QALY100 was used to identify which treatments represent best value for money (i.e. has highest NMB). The interactions for cost and QALYs combine to give a qualitative, but non-significant interaction for NMB $(p=0.100)$. Best-practice advice plus injection had a $54.93 \%$ probability of being the most cost-effective treatment at a ceiling

TABLE 25 Regression analysis with an interaction term, including imputation of missing values and adjustment for baseline utility, sex and age (base-case analysis)

\begin{tabular}{|c|c|c|c|}
\hline Regression analysis & $\begin{array}{l}\text { Total costs (£), } \\
\text { mean (SE) }\end{array}$ & QALYs, mean (SE) & NMB (E), ${ }^{a}$ mean (SE) \\
\hline BPA & $195(54)$ & $0.737(0.013)$ & $14,538(290)$ \\
\hline IBPA & $205(20)$ & $0.757(0.011)$ & $14,939(227)$ \\
\hline ProgEx & $247(23)$ & $0.756(0.012)$ & $14,865(255)$ \\
\hline IProgEx & $307(30)$ & $0.742(0.012)$ & $14,524(263)$ \\
\hline Injection simple effect (IBPA - BPA) & $10(44)(p=0.747)$ & $0.021(0.015)(p=0.184)$ & $402(322)(p=0.212)$ \\
\hline ProgEx simple effect (ProgEx - BPA) & $52(44)(p=0.247)$ & $0.019(0.016)(p=0.220)$ & $327(323)(p=0.309)$ \\
\hline $\begin{array}{l}\text { Interaction (BPA - IBPA - ProgEx + } \\
\text { IProgEx): ProgEx by injection }\end{array}$ & $50(57)(p=0.397)$ & $-0.035(0.022)(p=0.106)$ & $-743(455)(p=0.100)$ \\
\hline \multicolumn{4}{|c|}{$\begin{array}{l}\text { BPA, best-practice advice; IBPA, injection plus best-practice advice; IProgEx, injection plus progressive exercise; } \\
\text { ProgEx, progressive exercise. } \\
\text { a NMB calculated at a ceiling ratio of } £ 20,000 \text { per QALY. } \\
\text { Notes } \\
\text { Values represent the mean (SE) for each group for males of age } 55.46 \text { years and a baseline utility of } 0.653 \text {. As there } \\
\text { was assumed to be no interaction between baseline variables and treatments, the simple effects for each treatment } \\
\text { and the interaction between treatments are assumed to be the same for all participant subgroups, although the } \\
\text { absolute costs and absolute QALYs may be higher or lower, depending on participants' sex, age and baseline utility. }\end{array}$} \\
\hline
\end{tabular}


ratio of $£ 20,000$ per QALY (Figure 12). Progressive exercise alone had the second highest NMB and had a $35.64 \%$ probability of being cost-effective at a ceiling ratio of $£ 20,000$ per QALY.

\section{Sensitivity analyses}

The analysis ignoring the interaction between exercise treatment and injection also found it to be cost-effective to adopt injection, but not progressive exercise, although there remained substantial uncertainty around this conclusion (see Appendix 5, Table 54). In this analysis, progressive exercise (with/without injection) had a statistically significant effect on cost $(p=0.012)$, with an increase of $£ 78$ per participant compared with no progressive exercise (with/without injection), but gained $<0.001$ QALYs $(p=0.984)$. Progressive exercise, therefore, cost $£ 438,089$ per QALYs gained compared with no progressive exercise. Injection (with/without progressive exercise) non-significantly increased cost ( $£ 35$ per participant; $p=0.239$ ) while offering a negligible QALY gain $(0.003 ; p=0.818$ ), but was, nonetheless, expected to be cost-effective compared with no injection (with/without progressive exercise), costing $£ 15,110$ per QALY gained.

Appendix 5, Table 55, presents an extension to the NHS and PSS perspective taken by the base-case analysis to further consider the societal perspective, which included expenses borne by participants in the study, over-the-counter medication cost, income loss and cost of private care. In this analysis, adding injection to best-practice advice gained 0.02 QALYs $(p=0.183)$ and decreased the cost by $£ 34$ per participant $(p=0.813)$. Progressive exercise alone was $£ 60(p=0.247)$ less expensive per participant than best-practice advice, while offering a gain of 0.019 QALYs $(p=0.229)$. When injection was added to progressive exercise, it increased costs by $£ 374$ per participant, as there was a very large non-significant qualitative interaction for QALYs ( $£ 408 ; p=0.106)$. The interactions for cost and QALYs combined to give a qualitative and borderline statistically significant interaction for NMB $(p=0.049)$. Best-practice advice plus injection was expected to be the most cost-effective treatment (with a $47.55 \%$ probability of being cost-effective), followed by progressive exercise only (with a $44.87 \%$ probability of being cost-effective).

As described above, we extended the base-case analysis by considering the maximum cost of delivering the training for best-practice advice and progressive exercise (e.g. travel cost, venue hire, parking) while calculating the treatment cost of the intervention. In this analysis, the total cost of training was $£ 139.44$ per participant for best-practice advice and $£ 166.69$ per participant for progressive exercise. The results of the worst-case cost-effectiveness analysis are shown in Appendix 5, Table 56. QALY gains from the interventions are virtually identical to the base-case analysis (differing only from Monte Carlo error due to bootstrapping) and differences between treatment groups were not statistically significant.

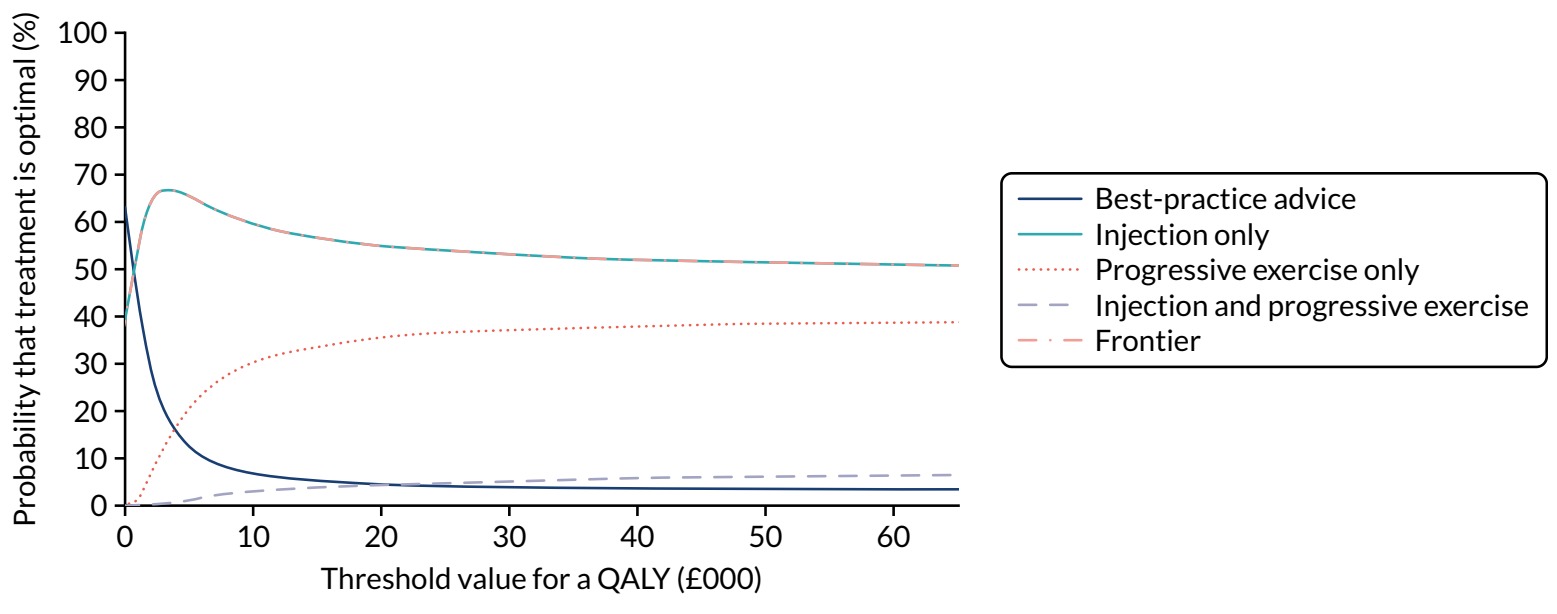

FIGURE 12 The CEAC for the comparison between treatment groups (base-case analysis). The frontier indicated which treatment is economically preferred at different threshold values for cost-effectiveness. 
The total training cost per participant was $£ 416.54$ higher than in the base case, although the incremental cost of progressive exercise compared with best-practice advice alone increased by only $£ 41$ compared with the base case. At a $£ 20,000$ per QALY ceiling ratio, best-practice advice plus injection remained the most cost-effective treatment, with a $53.59 \%$ probability of being cost-effective. Progressive exercise and progressive exercise plus injection were dominated (i.e. more costly and less effective) by best-practice advice plus injection.

Finally, we conducted a sensitivity analysis that assumed that study interventions would be implemented in routine clinical practice with the training delivered on an online platform. The best-case analysis results are presented in Appendix 5, Table 57. The total cost of training was $£ 2.35$ per participant for best-practice advice and $£ 3.02$ for progressive exercise, which is, respectively, $£ 40.85$ and $£ 46.41$ less expensive per participant than the base case. At a $£ 20,000$ per QALY ceiling ratio, best-practice advice plus injection remained the most cost-effective treatment, with a $53.6 \%$ probability of being cost-effective. Progressive exercise and progressive exercise plus injection were dominated (i.e. more costly and less effective) by best-practice advice plus injection.

\section{Discussion}

The GRASP trial economic analysis evaluated the cost-utility of best-practice advice alone, bestpractice advice preceded by a corticosteroid injection, progressive exercise alone and progressive exercise preceded by a corticosteroid injection, and compared alternative methodological approaches for conducting economic evaluation alongside factorial trials.

At a $£ 20,000$ per QALY ceiling ratio, a corticosteroid injection followed by best-practice advice was expected to be the most cost-effective treatment for people with a new episode of a rotator cuff disorder, regardless of the analytical approach adopted, although there was substantial uncertainty around this conclusion. There were no significant differences in costs or QALYs and there was a $36 \%$ chance that progressive exercise alone is the most cost-effective strategy. In addition, the strength of evidence in favour of best-practice advice plus injection did not vary considerably between the base-case analysis, ignoring interactions, and the two sensitivity analyses varying the assumptions about training cost. Across these analyses, NMBs for best-practice advice plus injection ranged from $£ 14,783$ to $£ 14,986$.

Although missing data are usually an issue in economic analysis and may introduce bias into the health economics results, the rate of complete response to the health-care resource use questions and EQ-5D questionnaire was reasonably high in the GRASP trial. Regardless, robust multiple imputation models have been applied and included all treatment indicators.

One of the advantages of conducting a factorial design trial is that it enables us to compare four treatment options within a single trial and explore whether or not there are interactions between treatments. We observed interactions for both costs and QALYs, which were very large compared with the main effect of treatment and meant that adding injections to best-practice advice increased mean QALYs, whereas adding injections to progressive exercise decreased mean QALYs. The opposite trend was observed for costs when a societal perspective was taken, which translated to a statistically significant interaction for NMB. We are not aware of a clinical mechanism that may explain these interactions and these interactions (only one of which was statistically significant) may have arisen by chance. Furthermore, even relatively small interactions will change the direction of clinical effect in studies such as this where the simple effect of treatment is very small. As randomised controlled trials, in general, are not powered based on economic end points but rather clinical outcomes, there is an ongoing argument that the economic evaluations are very likely underpowered. This argument is more prominent in a factorial design setting and increases the degree of uncertainty around the economic estimates in particular. We followed recent methodological work in prespecifying that the economic evaluation would include an interaction term regardless of statistical significance. ${ }^{88}$ In the base-case 
analysis, interactions between treatments were not statistically significant, suggesting that the standard statistical approach would have been to conduct an at-the-margins approach. Only when the broader societal perspective was taken together with the base-case NHS perspective did the interactions for cost and QALYs combine to give a qualitative and statistically significant interaction for NMB. However, our sensitivity analysis demonstrated that the conclusion that best-practice advice plus injection was the most cost-effective treatment remained unchanged in the sensitivity analysis omitting the interaction term. Dakin and Gray ${ }^{88}$ have discussed the challenges associated with the economic evaluations conducted alongside factorial trials, but further research is needed in this area.

The current economic evaluation used the newly developed EQ-5D-5L to capture the health-related quality of life for patients with a new episode of a rotator cuff disorder. It is regarded as more sensitive in capturing health changes than its predecessor (i.e. the EuroQol-5 Dimensions, three-level version). Nonetheless, as a generic measure of health, it is not disease specific and, hence, not expected to capture all the treatment benefits. The economic analysis showed a negligible and non-statistically significant QALY difference between the treatment groups, which is also consistent with the primary clinical outcome (i.e. SPADI) in this trial.

Finally, our economic evaluation included data from the GRASP trial only. Although there are no previous data on the specific progressive exercise regimen used in this study, there have been a number of previous studies of injections..$^{30}$ In principle, future analyses could use methods such as Bayesian bootstrapping $88,104,105$ to combine evidence from the GRASP trial with previous evidence on the efficacy of injection compared with no injection. 



\title{
Chapter 6 Discussion
}

\begin{abstract}
This chapter provides an overview of the aims of the GRASP trial and summarises the main findings, before considering its internal and external validity. The interpretation of the findings are then considered in the context of data from other similar trials in this area.
\end{abstract}

\section{Interpretation}

\section{Aim and overview of trial findings}

Shoulder pain in the UK is very common, with the most common attribution being the rotator cuff, which accounts for around $70 \%$ of new episodes of shoulder pain presenting in primary care. ${ }^{2}$ The majority of shoulder pain is managed in primary care or at primary care interface services by physiotherapists and GPs. Current treatments aim to improve pain and function with standard care that includes rest, advice, analgesia, physiotherapist-prescribed exercise and corticosteroid injection; however, there are no NICE clinical guidelines for this area. ${ }^{4}$ Prior to the GRASP trial, limited evidence existed regarding the most clinically effective and cost-effective form of physiotherapist-prescribed exercise and delivery mechanism associated with the best outcomes for people with a rotator cuff disorder. There was also uncertainty around the long-term benefits and harms associated with corticosteroid injection. In the GRASP trial, we aimed to assess the clinical effectiveness and cost-effectiveness of whether or not (1) an individually tailored progressive, home exercise programme prescribed and supervised by a physiotherapist provided greater improvement in shoulder pain and function over 12 months (measured using the SPADI score) compared with a best-practice advice session with a physiotherapist and provision of high-quality self-management materials, and (2) subacromial corticosteroid injection provided greater improvement in shoulder pain and function over 12 months compared with no injection.

For the primary outcome, there was no evidence of a difference in the SPADI scores over 12 months between participants randomised to receive the progressive-exercise intervention and those who received best-practice advice. Likewise, there was no evidence when analysed at the 8-week and 6-and 12-month time points. In both intervention groups, shoulder pain and function did improve over time, although SPADI scores at 12 months showed that the condition did not resolve completely, as most participants still reported some symptoms. There was also no difference between groups for secondary outcome measures, with the exception of progressive exercise, which resulted in an improvement in patientreported GIT over 12 months and at the 6- and 12-month time points. There were no significant subgroup differences in shoulder pain and function across the different time points when assessed for age, sex, baseline smoking status, SPADI and pain self-efficacy.

Over 12 months, there was no evidence of a difference in SPADI scores between participants randomised to receive corticosteroid injection and those receiving no injection, nor when analysed at the 6-and 12-month time points. There was a small difference in SPADI scores at 8 weeks, in favour of injection, but this was just below the threshold for a clinically important difference in the SPADI. There were no differences between groups for secondary outcome measures, with the exception of corticosteroid injection at 8 weeks, which resulted in a small improvement in shoulder pain, shoulder function, sleep disturbance, RDA and GIT. Prespecified subgroup analysis showed that the effect of corticosteroid injection was stronger at 8 weeks in people with a higher baseline SPADI score (i.e. SPADI $\geq 50$ ) than in those who received injection but had a lower baseline SPADI score. No differences were observed for other prespecified subgroup analyses.

At 8 weeks and 6 months, participants who received injections were more likely to report performing their exercises 5 days per week, in accordance with the advice from the treating physiotherapist, than participants who did not receive injections. This suggests that, although the effect of corticosteroid injection is short lived, it may facilitate engagement with prescribed home exercises. There were no SAEs recorded as part of the trial as a result of either corticosteroid injection or physiotherapy. 
A cost-utility analysis investigated the impact of progressive exercise, injection and the interaction between progressive-exercise treatment and injection, accounting for missing values and adjusting for baseline imbalance. There were no statistically significant differences between the treatment groups in terms of costs or QALYs over the 12-month period. More specifically, the addition of injection to bestpractice advice produced a negligible and non-statistically significant QALY gain, with a small extra cost. Progressive exercise was more expensive per participant than best-practice advice, but, again, with a negligible and non-statistically significant QALY gain. Progressive exercise alone and progressive exercise plus injection were both strongly dominated by best-practice advice plus injection, being more costly and accruing fewer QALYs. Among the treatments under investigation, the one expected to provide the best value for money was the best-practice advice session with a physiotherapist and injection, although there is substantial uncertainty around this conclusion. Although the benefits of corticosteroid injection were limited both in size and to the early phase of recovery, this combination of interventions dominated in terms of their cost-effectiveness.

\section{Generalisability}

\section{Internal validity and methodology}

The GRASP trial was a pragmatic multicentre superiority $2 \times 2$ factorial randomised controlled trial. Given the factorial design, we first formally tested for an interaction effect. In the absence of any significant interaction, we were able to assess the effects of our two main comparisons: (1) progressiveexercise programme compared with best-practice advice session and (2) subacromial corticosteroid injection compared with no injection. In accordance with the sample size estimation (see Chapter 4), data from 704 participants were required to detect a standardised effect size of 0.33 (equivalent to 8 points on the SPADI total score) with $90 \%$ power and $1 \%$ two-sided statistical significance, allowing for $20 \%$ loss to follow-up at 12 months and potential for a small clustering effect by physiotherapist. ${ }^{36}$ The DMEC reviewed the sample size assumptions after 338 participants had been recruited and did not recommend any changes to the final sample size. We recruited a total of 708 participants and had a lower than estimated loss to follow-up rate of $13 \%$ at 12 months, and so the trial was adequately powered to detect a statistically and clinically important difference between interventions. Measurements for the primary and secondary outcomes were collected by postal questionnaires at 8 weeks, 6 months and 12 months. When postal questionnaires were returned to the GRASP trial office, data were checked and if missing data were identified, either for part or a whole outcome measure, then the participant was contacted and information collected by telephone. As a result, we had limited missing outcome data from the completed questionnaires.

Randomisation was generated using the centralised computer randomisation service provided by OCTRU. Randomisation was stratified by site, age and sex using variable block size, ensuring that participants were balanced across interventions groups and minimising the chances of research staff anticipating treatment allocation prior to randomisation. It was not possible to blind participants and treating physiotherapists because of the nature of the interventions being tested. Both the primary and secondary outcomes were patient reported and collected using postal questionnaires. There was potential for bias as participants were aware of treatment allocation, but this reflects the difficultly of achieving and maintaining blinding in pragmatic rehabilitation trials of this nature. ${ }^{72}$ In the small number of cases where outcome data were collected by telephone (e.g. participants who did not respond to postal reminders), the researcher was blinded to the treatment allocated and participants were asked not to disclose which intervention they had received.

Physiotherapists were trained to deliver either the best-practice advice intervention or the progressiveexercise intervention to minimise possible contamination between treatment groups. Only two physiotherapists swapped treatment groups during the trial, because of staffing issues at sites, and 
so ended up delivering both interventions. According to the results of the GIT questionnaire, the interventions were generally well received by the participants. Interventions were also delivered with high levels of fidelity by the physiotherapists, as determined by quality assurance visits conducted at site and review of treatment logs. Attendance rates were $94 \%$ for injection, $95 \%$ for progressive exercise (either partially or full completed) and $92 \%$ for best-practice advice. These attendance rates are above those normally expected for NHS outpatient physiotherapy, with non-attendance rates estimated to be $10.4 \%$ according to recent NHS Hospital Episode Statistics data. ${ }^{41}$ There was no difference in attendance rates between those receiving the physiotherapist-delivered exercise interventions and those who received physiotherapist-delivered exercise interventions in conjunction with a corticosteroid injection.

Despite some initial concerns from physiotherapists raised during site training regarding the adequacy of a single contact with a physiotherapist to start a self-guided exercise programme, very few participants in the best-practice advice intervention required an additional contact session during the trial. Most participants in the progressive-exercise intervention group attended a median of four sessions (out of a maximum of six sessions) before being discharged. Progression of exercises, defined as an increase in exercise difficulty or volume and/or load, was regarded as key to achieving the overload and subsequent physiological response in the neuromuscular system to improve muscle function. Treatment logs provided evidence that $67 \%$ of participants in the progressive-exercise group had exercise progression between their first and last session, $22 \%$ maintained the initial exercise dose during treatment and only $12 \%$ had to regress their exercise dose over the sessions. The maintenance or regression of the level of exercise over the sessions was consistent with the tailoring and modification of the exercise programme allowed in the intervention protocol in the trial. The intervention was designed to enable therapists to adapt the programme according to the participant's response to exercising the shoulder.

A small proportion of participants (3.8\%) withdrew from the GRASP trial. This was either withdrawal from the intervention only or withdrawal from both the intervention and future follow-up assessment. The most common reason was a change in diagnosis since randomisation, and not dissatisfaction with treatment. Numbers were balanced across intervention groups. Participants were advised that they may seek other forms of treatment/medication outside the GRASP trial. This information was collected as part of the participant follow-up questionnaires. There were no significant differences in medication type or other health-care resources usage across the intervention groups, suggesting that the effects seen in terms of any improvement in shoulder pain and function were due to the trial interventions.

\section{External validity and generalisability of study findings}

The GRASP trial recruited participants from 20 primary care-based musculoskeletal services and their related physiotherapy services in the NHS. We had originally planned for eight sites; however, slower than anticipated recruitment meant that we increased our overall number of sites. The advantage of this was a greater range of centres in terms of geography and size, making it more representative of the NHS as a whole.

The training provided to the staff in these centres was relatively brief, with most NHS physiotherapy staff having expertise in delivering the best-practice advice intervention, with limited additional training. The best-practice advice intervention places emphasis on strategies to optimise self-management, with participants receiving a single face-to-face session rather than four to six sessions, as would be more typical of standard NHS physiotherapy treatment for this condition. As a result, we believe that the implementation of the best-practice advice intervention in the NHS would be straightforward and would involve relatively small training costs. No additional training was required for the subacromial corticosteroid injection, as this was delivered in accordance with current NHS practice and predominantly by extended-scope physiotherapists with the appropriate post-registration qualification in injection therapy. 
We believe that the participants recruited into the GRASP trial are representative of patients referred to NHS primary care-based musculoskeletal and physiotherapy services with a new episode of shoulder pain due to a rotator cuff disorder, in terms of their age and sex. Screening log data showed that the age and sex of patients who declined to take part in the trial were similar to those who did take part. However, one of the main reasons that participants declined to take part in the trial was because they were not interested in taking part in research $(30 \%)$ or that they had a treatment preference for not wanting to receive corticosteroid injection (27\%), as opposed to $7 \%$ having a treatment preference for wanting to receive injection. The extent to which this is representative of clinical practice outside a trial setting is unclear.

The mean age of participants recruited into the GRASP trial was 55.5 years, with a similar proportion of men and women. This is consistent with data from a large epidemiological study of rotator cuff pathology 106 using The Health Improvement Network database (a large UK primary care database) in which the highest incidence of rotator cuff pathology was found in those aged between 55 and 59 years, with no significant difference between men and women. This study also showed that people in the lowest socioeconomic group (with the Townsend deprivation score used as a measure of material deprivation) had the highest disease incidence. ${ }^{106}$ This is reflected in the findings of the GRASP trial, in which, despite around half of participants reporting being in employment, their reported household income was low.

Our population was predominantly white British (89.7\%) and this figure is higher than the population in England as a whole (78.7\%). ${ }^{107}$ The prevalence of rotator cuff disorder in ethnic minority groups is not well known or understood and so it is difficult to infer what influence this may have on the generalisability of our results. More than two-thirds of participants included in the GRASP trial were considered to be overweight or obese based on their body mass index. Again, the effect of obesity on the prevalence of rotator cuff disorders is not well known, including the extent to which it can contribute to symptoms ${ }^{108}$ and ability to exercise effectively. There is some evidence that obesity can lead to poorer outcomes after surgery to repair a rotator cuff tear. ${ }^{109}$

The mean duration of symptoms for participants included in the GRASP trial was 4 months. Our eligibility criteria meant that we specifically wanted to target those participants with a new episode of shoulder pain attributable to a rotator cuff and who had not received corticosteroid injection or physiotherapy for shoulder pain in the last 6 months. The overall mean SPADI baseline score was 54.1 (on a scale of 0-100), with higher reported levels of shoulder pain (mean 63.9) than impairment of shoulder function (mean 44.3). Irrespective of the allocated intervention, participant shoulder pain and function improved over time, although overall SPADI scores at 12 months (mean 21.9) show that symptoms did not resolve completely. These findings are consistent with a large epidemiological study of the prevalence of people consulting for shoulder pain in UK primary care, whereby $13.6 \%$ of people with shoulder pain continued to consult beyond 2 years from initial presentation. ${ }^{1}$ The SELF trial, which compared the effectiveness of usual physiotherapy treatment with a programme of self-management, also found that rotator cuff symptoms had not resolved completely when measured using the SPADI scale at 12 months. ${ }^{67}$

\section{Overall evidence: comparison with other literature}

\section{Exercise intervention}

Findings from a Cochrane review published in 2016, before the start of the GRASP trial, highlighted the lack of evidence about the long-term clinical effectiveness and cost-effectiveness of physiotherapy for the treatment of rotator cuff disorders, despite its widespread provision. ${ }^{14}$ Evidence from several small trials with short-term follow-up also raised uncertainty about which types of exercise and delivery mechanisms were associated with best outcomes. ${ }^{15,16}$

We searched MEDLINE, EMBASE and Cumulative Index to Nursing and Allied Health Literature (CINHAL) to identify new evidence relevant to the GRASP trial (date of last search: June 2020). 
From an initial 2354 records, we identified seven trials ${ }^{67,110-115}$ published between 2013 and 2020 , comparing the effects of supervised exercise with unsupervised exercise, or no intervention, in people with a rotator cuff disorder (excluding those who required surgery). Most of the trials concluded that there was little or no difference between supervised and unsupervised exercise. The populations were comparable to the GRASP trial, with the exception of the trial by Krischak et al., ${ }^{110}$ which evaluated people with atraumatic full-thickness rotator cuff tears. All trials were small, with the exception of the trial by Contreras et al. ${ }^{113}(n=271)$ and the SUPPORT trial ${ }^{115}$ [which had 64 participants in each group $(2 \times 2$ factorial trial) and 256 participants in total]. Only two ${ }^{67,115}$ of the seven trials reported on the effect of exercise on shoulder pain and function at 12 months, two reported medium-term follow-up data ( 24 or 26 weeks ${ }^{111,113}$ ) and the remaining three trials reported outcomes at $\leq 6$ weeks (see Appendix 6, Table 58). This reinforces the importance of the GRASP trial findings in terms of their definitive nature and length of follow-up.

\section{Corticosteroid injection}

At the time of planning the GRASP trial, there was systematic review evidence that, in comparison with placebo, corticosteroid injections had short-term benefit for treating tendinopathy, although there was some uncertainty regarding its use for rotator cuff disorders. There were also concerns about the longer-term safety of corticosteroid injection and its effect on the tendon. ${ }^{30}$

We searched MEDLINE, EMBASE, the Allied and Complementary Medicine Database (AMED), CINHAL, the Cochrane Central Register of Controlled Trials (CENTRAL), ClinicalTrials.gov and the World Health Organization International Clinical Trials Registry to identify new evidence relevant to the GRASP trial (date of last search: June 2020)..${ }^{91}$ From an initial 794 records, we identified one small trial 116 that compared the effects of corticosteroid injection with no injection in people with a rotator cuff tear and found no difference in shoulder pain and function when analysed at 3 or 6 months, measured using the Constant-Murley score (MD at 6 months $-1.9,95 \% \mathrm{Cl}-10.89$ to 7.09 ; patients, $n=40$ ).

We identified an additional 10 trials $7,90,117-124$ that compared the effects of corticosteroid injection with placebo injection (see Appendix 6, Table 59), of which four $7,118,121,122$ were judged as suitable for inclusion in a meta-analysis. The remaining studies could not be included because of either incomplete or incompatible outcome data. Three trials, ${ }^{118,121,122}$ with a total of 215 patients, compared subacromial corticosteroid injection with placebo injection for combined shoulder pain and function in the short term (i.e. $\leq 8$ weeks), with results favouring corticosteroid over placebo [standardised mean difference (SMD) $-0.51,95 \% \mathrm{Cl}-1.02$ to $0.00 ; I^{2}=69 \% ; n=3$; patients, $n=215$; rated as having moderate-quality evidence]. Two of the trials ${ }^{118,122}$ also reported outcome data for medium-term follow-up (i.e. 3-6 months), in which no difference was apparent (SMD $0.08,95 \% \mathrm{Cl}-0.24$ to $0.39 ; 1^{2}=0 \% ; n=2$; patients, $n=151$; rated as having moderate-quality evidence). With regard to shoulder pain only, three trials reported short-term outcomes ${ }^{118,121,122}$ and two reported medium-term outcomes. ${ }^{118,122}$ The results mirrored those seen for combined shoulder pain and function, which favoured corticosteroid injection over placebo in the short term (SMD $-0.35,95 \% \mathrm{Cl}-0.65$ to $-0.05 ; I^{2}=17 \% ; n=3$; patients, $n=215$; rated as having moderate quality evidence), but not the medium term (SMD $0.05,95 \% \mathrm{Cl}-0.27$ to $0.37 ; 1^{2}=0 \% ; n=2$; patients, $n=151$; rated as having moderate-quality evidence). Two trials ${ }^{7,122}$ reported on shoulder functional improvement in the short and medium term and, again, there was a short-term benefit favouring corticosteroid injection (SMD $-0.33,95 \% \mathrm{Cl}-0.67$ to $0.00 ; 1^{2}=0 \% ; n=2$; patients, $n=143$; rated as having moderate-quality evidence), but there was no difference seen in medium-term functional outcomes (SMD $-0.24,95 \% \mathrm{Cl}-0.59$ to $0.10 ; l^{2}=0 \% ; n=2$; patients, $n=131$; rated as having moderate-quality evidence). No trials provided outcome data beyond 6-month follow-up and none reported any SAEs as a result of injection.

These findings reinforce the importance of the results of the GRASP trial in terms of the short-term benefit of subacromial corticosteroid injection, a benefit which is not maintained at 6- and 12-month follow-up. 



\section{Chapter 7 Conclusion} his chapter provides interpretation of the GRASP trial findings for clinical practice and policy,
and provides recommendations for future research.

In adults with a new episode of shoulder pain (i.e. within the last 6 months) attributable to a rotator cuff disorder, there was no difference in the primary outcome (SPADI) or other prespecified secondary outcomes between participants randomised to receive (1) progressive exercise compared with bestpractice advice, or (2) subacromial corticosteroid injection compared with no injection, when analysed over 12 months. Irrespective of allocated intervention, participants' shoulder pain and function improved over time, although SPADI scores at 12 months show that the condition did not resolve completely, as participants still reported some symptoms. Participants randomised to receive subacromial corticosteroid injection reported an improvement in shoulder pain and function at 8 weeks, but injection provided modest short-term benefit only. The greatest benefit of injection was seen in the subgroup of participants who reported higher SPADI scores at baseline; however, as this is based on subgroup analysis, this should be viewed with caution. No SAEs were reported as a result of the intervention(s). The cost-utility analysis, performed as part of the GRASP trial, suggests that, at a $£ 20,000$ per QALY ceiling ratio, a corticosteroid injection followed by best-practice advice is likely to be the most cost-effective treatment combination for people with a new episode of a rotator cuff disorder. There are no significant differences in costs or QALYs.

\section{Implications for health care}

Early and effective management of rotator cuff disorders in primary care and primary care interface musculoskeletal services is vital, given their associated disability and commonality. Consequences of poor initial management may lead to an increased likelihood of recurrent or persistent problems in older age and the subsequent need for surgery. This is particularly important given recent evidence from the NIHR HTA-funded CSAW trial ${ }^{125}$ and updated Cochrane review ${ }^{93}$ that showed a lack of benefit from subacromial decompression surgery, which is often used after non-operative interventions have failed.

The GRASP trial shows that a single face-to-face session with a physiotherapist is likely to be more cost-effective and is not significantly different in terms of clinical outcomes than a comprehensive physiotherapy intervention of up to six face-to-face sessions. This is particularly important given the incidence of rotator cuff disorders and the need to develop cost-effective and pragmatic methods of dealing with this high-volume condition. Subacromial corticosteroid injection provides a modest short-term benefit and is associated with an increased level of engagement in exercise. The greatest benefit was observed in those with higher levels of pain and functional impairment at baseline.

Physiotherapists delivering the best-practice advice intervention attended a short face-to-face training session where focus was on strategies to promote self-management and independent progression of exercise, adherence to exercise and addressing barriers to exercise. The exercises prescribed were those within the range that physiotherapists deliver in usual practice. We are exploring the use of an online training module where physiotherapists can access the best-practice advice intervention training virtually. This has proved very successful in implementation of physiotherapy interventions from other musculoskeletal trials. ${ }^{45}$ In addition, the materials provided to participants in terms of the best-practice advice booklet, exercise DVD and website are in English and, therefore, there is a need to explore translation of these materials into other languages. There may also be some people for whom a single session is not appropriate, for example those with low levels of literacy or inability to engage with self-management care, in which case additional physiotherapy sessions may be required. 


\section{Implications for research}

There is a case to extend follow-up beyond 12 months, as some participants still reported pain and impaired shoulder function at 12 months. There is a need to better understand the natural history of rotator cuff disorders, including whether symptoms resolve over an extended period of time or persist in the longer term. Part of this investigation could involve studies examining the natural course of rotator cuff disorders and could include a randomised trial that compares the effects of no physiotherapy with best-practice advice.

Although subacromial corticosteroid injection provided short-term benefit, there are still concerns regarding long-term harm due to its possible effects on tendon structure. ${ }^{126}$ Very few participants reported having undergone surgery related to their rotator cuff disorder during the 12-month follow-up period. Twelve months may be too early to measure this effectively, as participants may still be undergoing other forms of non-operative treatment. Therefore, longer-term follow-up would be beneficial.

Finally, screening data from trial sites as part of the recruitment process showed that some people had a clear preference not to receive corticosteroid injection and, therefore, declined to take part in the trial. The reason for this strong treatment preference is not clear and would warrant further investigation to establish the reasons why. 


\section{Acknowledgements}

\section{GRASP trial management team}

Sally Hopewell (co-chief investigator), Lucy Cureton (trial manager), Adam Pritchard (trial administrator), Peter Heine (Research Physiotherapist), David J Keene (lead physiotherapist), loana R Marian (statistician), Melina Dritsaki (health economist) and Sarah E Lamb (co-chief investigator).

We would like to thank Debbie Brown (Research Physiotherapist) for her assistance with development of the GRASP trial exercise interventions, Hessam Soutakbar (Research Physiotherapist) for his assistance with GRASP trial exercise intervention site training and monitoring and Jacqueline Thompson for her assistance with GRASP trial participant telephone follow-up. We would also like to thank Emma Searle (MSc student; Université Paris Descartes, Paris, France) for her help with updating the literature search.

\section{Principal investigators and research teams by site}

We are grateful to the participants, principal investigators and their research teams at our GRASP trial collaborating sites.

University Hospitals of Derby and Burton NHS Foundation Trust

Marcus Bateman (principal investigator).

East Lancashire Hospitals NHS Trust

Alison Hallett (principal investigator) and Helen Thompson.

Gloucestershire Hospitals NHS Foundation Trust

Elaine Willmore (principal investigator).

Birmingham Community Healthcare NHS Trust

Lucy McCann (principal investigator) and Jonathan Price.

Sandwell and West Birmingham Hospitals NHS Trust

Neil Smith (principal investigator).

Buckinghamshire Musculoskeletal Integrated Care Service

Harry Kardamilas (principal investigator).

East Cheshire Hospitals NHS Trust

Matt Hurst (principal investigator).

Bedfordshire Hospitals NHS Foundation Trust

Tim Andrews (principal investigator) and Lori Wells.

Wirral University Teaching Hospital NHS Foundation Trust

Chole De Matas (principal investigator).

Medway Community Healthcare

Arun Jaykumar (principal investigator). 


\section{Bristol Community Health}

Sean Grove (principal investigator).

Somerset Partnership NHS Foundation Trust

Corinne Birch (principal investigator).

Doncaster and Bassetlaw Teaching Hospitals NHS Foundation Trust

Julie Bury (principal investigator).

Sherwood Forest Hospitals NHS Foundation NHS Foundation Trust

James Blacknall (principal investigator).

Kent Community Health NHS Foundation Trust

Sally Jessep (principal investigator) and Llewelyn Boucher.

Northern Devon Healthcare NHS Trust

Robert Sandbach (principal investigator).

Airedale NHS Foundation Trust

Stacey Lalande (principal investigator).

North West Boroughs Healthcare NHS Foundation Trust

Gill Dickson (principal investigator).

Staffordshire and Stoke on Trent Partnership NHS Foundation Trust

Treena Larkin (principal investigator).

Warrington and Halton Hospitals NHS Foundation Trust

Carole Cummings (principal investigator).

\section{Trial Steering Committee}

Dr Neal Millar (chairperson, Clinical Senior Research Fellow), Dr Chris Metcalfe (Reader in Medical Statistics), Professor Rebecca Kearney (Research Physiotherapist), Dr Melinda Cairns (Consultant Physiotherapist), Dr Sue Jowett (Reader in Health Economics), Mr Richard Goodier (patient representative) and Dr Jean Millar (patient representative).

\section{Data Monitoring and Ethics Committee}

Professor Mike Clarke (chairperson, Independent Methodologist), Dr Brennan Kahan (Independent Statistician), Dr Alison Rushton (Independent Physiotherapist) and Dr Jessica Watson (Independent GP Registrar).

\section{Other acknowledgements}

The GRASP trial was conducted as part of the portfolio of trials in the registered United Kingdom Clinical Research Collaboration OCTRU at the University of Oxford. It has followed their standard operating procedures, ensuring compliance with the principles of GCP and the Declaration of Helsinki, and any applicable regulatory requirements. 
This research is funded by the NIHR HTA programme (15/26/06). The funder had no role in the design of the study, the collection, analysis and interpretation of data, and in writing the manuscript.

\section{Contributions of authors}

Sally Hopewell (https://orcid.org/0000-0002-6881-6984) (Associate Professor, University of Oxford) was the co-chief investigator; led the funding application, study conception and design; provided overall study supervision; and prepared and reviewed the report.

David J Keene (https://orcid.org/0000-0001-7249-6496) (University Research Lecturer, University of Oxford) contributed to study design; led on physiotherapy expertise-based design; advised on overall study conduct, review and preparation; and reviewed the report.

Peter Heine (https://orcid.org/0000-0003-2530-1216) (Research Physiotherapist, University of Oxford) conducted site physiotherapist training, quality assessment visits and site monitoring; conducted physiotherapy intervention analysis; and contributed to preparation and review of the report.

Ioana R Marian (https://orcid.org/0000-0002-0692-8112) (Trial Statistician, University of Oxford) conducted the clinical analysis and contributed to preparation and review of the report.

Melina Dritsaki (https://orcid.org/0000-0002-1673-3036) (Health Economist, University of Oxford) conducted the health economic evaluation and contributed to preparation and review of the report.

Lucy Cureton (https://orcid.org/0000-0003-0082-8468) (Trial Manager, University of Oxford) contributed to overall study conduct and preparation and review of the report.

Susan J Dutton (https://orcid.org/0000-0003-4573-5257) (Associate Professor, University of Oxford) was responsible for overseeing the statistical aspects of the study, contributed to study design and advised on expertise-based design, overall study conduct and review of statistical aspects, and preparation and review of the report.

Helen Dakin (https://orcid.org/0000-0003-3255-748X) (University Research Lecturer, University of Oxford) was responsible for overseeing the health economic evaluation and contributed to overall study design, and preparation and review of the report.

Andrew Carr (https://orcid.org/0000-0001-5940-1464) (Professor of Orthopaedic Surgery, University of Oxford) contributed to study design and advised on clinical aspects of trial, overall study conduct and review of the report.

Willie Hamilton (https://orcid.org/0000-0003-1611-1373) (Professor of Primary Care Diagnostics, University of Exeter) contributed to study design and advised on clinical aspects of trial, overall study conduct and review of the report.

Zara Hansen (https://orcid.org/0000-0001-8915-7765) (Post-Doctoral Researcher, University of Oxford) contributed to study design and advised on clinical aspects of trial, overall study conduct and review of the report.

Anju Jaggi (https://orcid.org/0000-0001-8413-2622) (Consultant Physiotherapist, Royal National Orthopaedic Hospital) contributed to study design and advised on clinical aspects of trial, overall study conduct and review of the report. 
Chris Littlewood (https://orcid.org/0000-0002-7703-727X) (Professor of Musculoskeletal Research, Manchester Metropolitan University) contributed to study design and advised on clinical aspects of trial, overall study conduct and review of the report.

Karen Barker (https://orcid.org/0000-0001-9363-0383) (Professor of Physiotherapy, University of Oxford) contributed to study design and advised on clinical aspects of trial, overall study conduct and review of the report.

Alastair Gray (https://orcid.org/0000-0003-0239-7278) (Professor of Health Economics, University of Oxford) contributed to study design and advised on health economic aspects of trial, overall study conduct and review of the report.

Sarah E Lamb (https://orcid.org/0000-0003-4349-7195) (Professor, University of Exeter) was the co-chief investigator, led the funding application, study conception and design, provided overall study supervision, and prepared and reviewed the report.

\section{Publication}

Hopewell S, Keene DJ, Marian IR, Dritsaki M, Heine O, Cureton L, et al. Progressive exercise compared with best practice advice, with or without corticosteroid injection, for the treatment of patients with rotator cuff disorders (GRASP): a multicentre, pragmatic, $2 \times 2$ factorial, randomised controlled trial [published online ahead of print 12 July 2021]. Lancet 2021.

\section{Data-sharing statement}

Direct access to research data was granted to authorised representatives of the sponsor, regulatory authorities or the host institution for monitoring and/or auditing of the study to ensure compliance with regulations. Summary results data will be included on the EudraCT database (URL: https://eudract.ema. europa.eu/) within 12 months of the end of the trial. General release will be 5 years after the end of the trial to allow the investigators sufficient time to complete and report additional analyses of the data set. All data requests should be submitted to the corresponding author.

\section{Patient data}

This work uses data provided by patients and collected by the NHS as part of their care and support. Using patient data is vital to improve health and care for everyone. There is huge potential to make better use of information from people's patient records, to understand more about disease, develop new treatments, monitor safety, and plan NHS services. Patient data should be kept safe and secure, to protect everyone's privacy, and it's important that there are safeguards to make sure that it is stored and used responsibly. Everyone should be able to find out about how patient data are used. \#datasaveslives You can find out more about the background to this citation here: https://understandingpatientdata.org.uk/data-citation. 


\section{References}

1. Linsell L, Dawson J, Zondervan K, Rose P, Randall T, Fitzpatrick R, Carr A. Prevalence and incidence of adults consulting for shoulder conditions in UK primary care; patterns of diagnosis and referral. Rheumatology 2006;45:215-21. https://doi.org/10.1093/rheumatology/kei139

2. Mitchell C, Adebajo A, Hay E, Carr A. Shoulder pain: diagnosis and management in primary care. BMJ 2005;331:1124-8. https://doi.org/10.1136/bmj.331.7525.1124

3. Whittle S, Buchbinder R. In the clinic. Rotator cuff disease. Ann Intern Med 2015;162:Itc1-15. https://doi.org/10.7326/AITC201501060

4. The Royal College of Surgeons. Commissioning Guide: Subacromial Shoulder Pain. 2014. URL: www.rcseng.ac.uk/library-and-publications/rcs-publications/docs/subacromial-shoulder-pain/ (accessed 24 May 2021).

5. National Institute for Health and Care Excellence. Shoulder Pain. 2017. URL: http://cks.nice.org.uk/ shoulder-pain\#!scenario:1 (accessed 2 March 2021).

6. Hermans J, Luime JJ, Meuffels DE, Reijman M, Simel DL, Bierma-Zeinstra SM. Does this patient with shoulder pain have rotator cuff disease? The Rational Clinical Examination systematic review. JAMA 2013;310:837-47. https://doi.org/10.1001/jama.2013.276187

7. Holt TA, Mant D, Carr A, Gwilym S, Beard D, Toms C, et al. Corticosteroid injection for shoulder pain: single-blind randomized pilot trial in primary care. Trials 2013;14:425. https://doi.org/10.1186/1745-6215-14-425

8. Urwin M, Symmons D, Allison T, Brammah T, Busby H, Roxby M, et al. Estimating the burden of musculoskeletal disorders in the community: the comparative prevalence of symptoms at different anatomical sites, and the relation to social deprivation. Ann Rheum Dis 1998;57:649-55. https://doi.org/10.1136/ard.57.11.649

9. Chartered Society for Physiotherapy. An Audit of Physiotherapy Services in England. 2012. URL: www.csp.org.uk/system/files/documents/2018-11/stretched-to-the-limit-2012.pdf (accessed 24 May 2021).

10. Roach KE, Budiman-Mak E, Songsiridej N, Lertratanakul Y. Development of a shoulder pain and disability index. Arthritis Care Res 1991;4:143-9. https://doi.org/10.1002/art.1790040403

11. Williams JW, Holleman DR, Simel DL. Measuring shoulder function with the Shoulder Pain and Disability Index. J Rheumatol 1995;22:727-32.

12. Hanratty CE, McVeigh JG, Kerr DP, Basford JR, Finch MB, Pendleton A, Sim J. The effectiveness of physiotherapy exercises in subacromial impingement syndrome: a systematic review and meta-analysis. Semin Arthritis Rheum 2012;42:297-316. https://doi.org/10.1016/ j.semarthrit.2012.03.015

13. Littlewood C, Ashton J, Chance-Larsen K, May S, Sturrock B. Exercise for rotator cuff tendinopathy: a systematic review. Physiotherapy 2012;98:101-9. https://doi.org/10.1016/ j.physio.2011.08.002

14. Page MJ, Green S, McBain B, Surace SJ, Deitch J, Lyttle N, et al. Manual therapy and exercise for rotator cuff disease. Cochrane Database Syst Rev 2016;6:CD012224. https://doi.org/ 10.1002/14651858.CD012224

15. Bennell K, Wee E, Coburn S, Green S, Harris A, Staples M, et al. Efficacy of standardised manual therapy and home exercise programme for chronic rotator cuff disease: randomised placebo controlled trial. BMJ 2010;340:c2756. https://doi.org/10.1136/bmj.c2756 
16. Littlewood C, Malliaras P, Mawson S, May S, Walters SJ. Self-managed loaded exercise versus usual physiotherapy treatment for rotator cuff tendinopathy: a pilot randomised controlled trial. Physiotherapy 2014;100:54-60. https://doi.org/10.1016/j.physio.2013.06.001

17. Ingwersen KG, Christensen R, Sørensen L, Jørgensen HR, Jensen SL, Rasmussen S, et al. Progressive high-load strength training compared with general low-load exercises in patients with rotator cuff tendinopathy: study protocol for a randomised controlled trial. Trials 2015;16:27. https://doi.org/10.1186/s13063-014-0544-6

18. Abdulla SY, Southerst D, Côté P, Shearer HM, Sutton D, Randhawa K, et al. Is exercise effective for the management of subacromial impingement syndrome and other soft tissue injuries of the shoulder? A systematic review by the Ontario Protocol for Traffic Injury Management (OPTIMa) Collaboration. Man Ther 2015;20:646-56. https://doi.org/10.1016/ j.math.2015.03.013

19. Littlewood C, Malliaras P, Chance-Larsen K. Therapeutic exercise for rotator cuff tendinopathy: a systematic review of contextual factors and prescription parameters. Int J Rehabil Res 2015;38:95-106. https://doi.org/10.1097/MRR.0000000000000113

20. Kraemer WJ, Adams K, Cafarelli E, Dudley GA, Dooly C, Feigenbaum MS, et al. American College of Sports Medicine position stand. Progression models in resistance training for healthy adults. Med Sci Sports Exerc 2002;34:364-80. https://doi.org/10.1097/00005768200202000-00027

21. Heine PJ, Williams MA, Williamson E, Bridle C, Adams J, O'Brien A, et al. Development and delivery of an exercise intervention for rheumatoid arthritis: strengthening and stretching for rheumatoid arthritis of the hand (SARAH) trial. Physiotherapy 2012;98:121-30. https://doi.org/ 10.1016/j.physio.2011.03.001

22. Green S, Buchbinder R, Hetrick S. Acupuncture for shoulder pain. Cochrane Database Syst Rev 2005;2:CD005319. https://doi.org/10.1002/14651858.CD005319

23. Green S, Buchbinder R, Hetrick S. Physiotherapy interventions for shoulder pain. Cochrane Database Syst Rev 2003;2:CD004258. https://doi.org/10.1002/14651858.CD004258

24. Jack K, McLean SM, Moffett JK, Gardiner E. Barriers to treatment adherence in physiotherapy outpatient clinics: a systematic review. Man Ther 2010;15:220-8. https://doi.org/10.1016/ j.math.2009.12.004

25. Sluijs EM, Kok GJ, van der Zee J. Correlates of exercise compliance in physical therapy. Phys Ther 1993;73:771-82. https://doi.org/10.1093/ptj/73.11.771

26. UK BEAM Trial Team. United Kingdom back pain exercise and manipulation (UK BEAM) randomised trial: effectiveness of physical treatments for back pain in primary care. BMJ 2004;329:1377. https://doi.org/10.1136/bmj.38282.669225.AE

27. Knox CR, Lall R, Hansen Z, Lamb SE. Treatment compliance and effectiveness of a cognitive behavioural intervention for low back pain: a complier average causal effect approach to the BeST data set. BMC Musculoskelet Disord 2014;15:17. https://doi.org/10.1186/1471-2474-15-17

28. Arroll B, Goodyear-Smith F. Corticosteroid injections for painful shoulder: a meta-analysis. Br J Gen Pract 2005;55:224-8.

29. Buchbinder R, Green S, Youd JM. Corticosteroid injections for shoulder pain. Cochrane Database Syst Rev 2003;1:CD004016. https://doi.org/10.1002/14651858.CD004016

30. Coombes BK, Bisset L, Vicenzino B. Efficacy and safety of corticosteroid injections and other injections for management of tendinopathy: a systematic review of randomised controlled trials. Lancet 2010;376:1751-67. https://doi.org/10.1016/S0140-6736(10)61160-9 
31. Crawshaw DP, Helliwell PS, Hensor EM, Hay EM, Aldous SJ, Conaghan PG. Exercise therapy after corticosteroid injection for moderate to severe shoulder pain: large pragmatic randomised trial. BMJ 2010;340:c3037. https://doi.org/10.1136/bmj.c3037

32. Rhon DI, Boyles RB, Cleland JA. One-year outcome of subacromial corticosteroid injection compared with manual physical therapy for the management of the unilateral shoulder impingement syndrome: a pragmatic randomized trial. Ann Intern Med 2014;161:161-9. https://doi.org/10.7326/M13-2199

33. Hay EM, Thomas E, Paterson SM, Dziedzic K, Croft PR. A pragmatic randomised controlled trial of local corticosteroid injection and physiotherapy for the treatment of new episodes of unilateral shoulder pain in primary care. Ann Rheum Dis 2003;62:394-9. https://doi.org/ 10.1136/ard.62.5.394

34. National Institute for Health and Care Excellence. Patient Group Directions. Medicines Practice Guideline [MPG2]. URL: www.nice.org.uk/guidance/mpg2 (accessed 20 May 2021).

35. Roddy E, Zwierska I, Hay EM, Jowett S, Lewis M, Stevenson K, et al. Subacromial impingement syndrome and pain: protocol for a randomised controlled trial of exercise and corticosteroid injection (the SUPPORT trial). BMC Musculoskelet Disord 2014;15:81. https://doi.org/10.1186/ 1471-2474-15-81

36. Hopewell S, Keene DJ, Maia Schlüssel M, Dritsaki M, Dutton S, Carr A, et al. Clinical and cost-effectiveness of progressive exercise compared with best practice advice, with or without corticosteroid injection, for the treatment of rotator cuff disorders: protocol for a 2 × 2 factorial randomised controlled trial (the GRASP trial). BMJ Open 2017;7:e018004. https://doi.org/10.1136/bmjopen-2017-018004

37. Marian IR, Hopewell S, Keene DJ, Cureton L, Lamb SE, Dutton SJ. Progressive exercise compared with best practice advice, with or without corticosteroid injection, for the treatment of rotator cuff disorders: statistical analysis plan for the Getting it Right: Addressing Shoulder Pain (GRASP) $2 \times 2$ factorial multicentre randomised controlled trial. Trials 2020;21:767. https://doi.org/10.1186/s13063-020-04704-5

38. Brooks R. EuroQol: the current state of play. Health Policy 1996;37:53-72. https://doi.org/ 10.1016/0168-8510(96)00822-6

39. Mintken PE, Cleland JA, Whitman JM, George SZ. Psychometric properties of the Fear-Avoidance Beliefs Questionnaire and Tampa Scale of Kinesiophobia in patients with shoulder pain. Arch Phys Med Rehabil 2010;91:1128-36. https://doi.org/10.1016/j.apmr.2010.04.009

40. Nicholas MK, McGuire BE, Asghari A. A 2-item short form of the Pain Self-efficacy Questionnaire: development and psychometric evaluation of PSEQ-2. J Pain 2015;16:153-63. https://doi.org/10.1016/j.jpain.2014.11.002

41. Bastien $\mathrm{CH}$, Vallières $\mathrm{A}$, Morin $\mathrm{CM}$. Validation of the Insomnia Severity Index as an outcome measure for insomnia research. Sleep Med 2001;2:297-307. https://doi.org/10.1016/ S1389-9457(00)00065-4

42. Kamper SJ, Maher CG, Mackay G. Global rating of change scales: a review of strengths and weaknesses and considerations for design. J Man Manip Ther 2009;17:163-70. https://doi.org/ 10.1179/jmt.2009.17.3.163

43. Keene DJ, Soutakbar H, Hopewell S, Heine P, Jaggi A, Littlewood C, et al. Development and implementation of the physiotherapy-led exercise interventions for the treatment of rotator cuff disorders for the 'Getting it Right: Addressing Shoulder Pain' (GRASP) trial. Physiotherapy 2020;107:252-66. https://doi.org/10.1016/j.physio.2019.07.002

44. Electronic Medicines Compendium. Electronic Medicines Compendium. URL: www.medicines.org. uk/emc/ (accessed 20 May 2021). 
45. Lamb SE, Hansen Z, Lall R, Castelnuovo E, Withers EJ, Nichols V, et al. Group cognitive behavioural treatment for low-back pain in primary care: a randomised controlled trial and cost-effectiveness analysis. Lancet 2010;375:916-23. https://doi.org/10.1016/S0140-6736(09) 62164-4

46. American College of Sports Medicine. American College of Sports Medicine position stand. Progression models in resistance training for healthy adults. Med Sci Sports Exerc 2009;41:687-708. https://doi.org/10.1249/MSS.0b013e3181915670

47. Jones R, Jenkins F. Safe and Effective Staffing Levels for the Allied Health Professions: A Practical Guide. Bristol: Otmoor Publishing Ltd; 2014.

48. Page MJ, McKenzie JE, Green SE, Beaton DE, Jain NB, Lenza M, et al. Core domain and outcome measurement sets for shoulder pain trials are needed: systematic review of physical therapy trials. J Clin Epidemiol 2015;68:1270-81. https://doi.org/10.1016/j.jclinepi.2015.06.006

49. Great Britain. Data Protection Act 2018. London: The Stationery Office; 2018.

50. Breckenridge JD, McAuley JH. Shoulder Pain and Disability Index (SPADI). J Physiother 2011;57:197. https://doi.org/10.1016/S1836-9553(11)70045-5

51. Lamb SE, Williamson EM, Heine PJ, Adams J, Dosanjh S, Dritsaki M, et al. Exercises to improve function of the rheumatoid hand (SARAH): a randomised controlled trial. Lancet 2015;385:421-9. https://doi.org/10.1016/S0140-6736(14)60998-3

52. Kahan BC. Bias in randomised factorial trials. Stat Med 2013;32:4540-9. https://doi.org/ 10.1002/sim.5869

53. Brown H, Prescott R. Applied Mixed Models in Medicine. 3rd edn. Chichester: John Wiley \& Sons, Ltd; 2014. https://doi.org/10.1002/9781118778210

54. Eekhout I, de Vet HC, Twisk JW, Brand JP, de Boer MR, Heymans MW. Missing data in a multi-item instrument were best handled by multiple imputation at the item score level. J Clin Epidemiol 2014;67:335-42. https://doi.org/10.1016/j.jclinepi.2013.09.009

55. Shirley Ryan AbilityLab. Shoulder Pain and Disability Index. 2015. URL: www.sralab.org/rehabilitationmeasures/shoulder-pain-and-disability-index\#chronic-pain (accessed 4 October 2019).

56. Sullivan TR, White IR, Salter AB, Ryan P, Lee KJ. Should multiple imputation be the method of choice for handling missing data in randomized trials? Stat Method Med Res 2018;27:2610-26. https://doi.org/10.1177/0962280216683570

57. Chester R, Shepstone L, Daniell H, Sweeting D, Lewis J, Jerosch-Herold C. Predicting response to physiotherapy treatment for musculoskeletal shoulder pain: a systematic review. BMC Musculoskelet Disord 2013;14:203. https://doi.org/10.1186/1471-2474-14-203

58. Tempelhof S, Rupp S, Seil R. Age-related prevalence of rotator cuff tears in asymptomatic shoulders. J Shoulder Elbow Surg 1999;8:296-9. https://doi.org/10.1016/S1058-2746(99)90148-9

59. Carbone S, Gumina S, Arceri V, Campagna V, Fagnani C, Postacchini F. The impact of preoperative smoking habit on rotator cuff tear: cigarette smoking influences rotator cuff tear sizes. J Shoulder Elbow Surg 2012;21:56-60. https://doi.org/10.1016/j.jse.2011.01.039

60. National Institute for Health and Care Excellence. Guide to the Methods of Technology Appraisal 2013. 2013. URL: www.nice.org.uk/article/pmg9/chapter/Foreword (accessed 2 March 2021).

61. World Medical Association. WMA Declaration of Helsinki - Ethical Principles for Medical Research Involving Human Subjects. URL: www.wma.net/policies-post/wma-declaration-of-helsinki-ethicalprinciples-for-medical-research-involving-human-subjects/ (accessed 15 July 2021). 
62. Medical Research Council. Good Research Practice: Principles and Guidelines. URL: https://mrc. ukri.org/research/policies-and-guidance-for-researchers/good-research-practice/ (accessed 15 July 2021).

63. Craig P, Dieppe P, Macintyre S, Michie S, Nazareth I, Petticrew M, Medical Research Council Guidance. Developing and evaluating complex interventions: the new Medical Research Council guidance. BMJ 2008;337:a1655. https://doi.org/10.1136/bmj.a1655

64. Edwards P, Ebert J, Joss B, Bhabra G, Ackland T, Wang A. Exercise rehabilitation in the non-operative management of rotator cuff tears: a review of the literature. Int $J$ Sports Phys Ther 2016;11:279-301.

65. Ratcliffe E, Pickering S, McLean S, Lewis J. Is there a relationship between subacromial impingement syndrome and scapular orientation? A systematic review. $\mathrm{Br} J$ Sports Med 2014;48:1251-6. https://doi.org/10.1136/bjsports-2013-092389

66. Klintberg IH, Cools AM, Holmgren TM, Holzhausen AC, Johansson K, Maenhout AG, et al. Consensus for physiotherapy for shoulder pain. Int Orthop 2015;39:715-20. https://doi.org/ 10.1007/s00264-014-2639-9

67. Littlewood C, Bateman M, Brown K, Bury J, Mawson S, May S, Walters SJ. A self-managed single exercise programme versus usual physiotherapy treatment for rotator cuff tendinopathy: a randomised controlled trial (the SELF study). Clin Rehabil 2016;30:686-96. https://doi.org/ 10.1177/0269215515593784

68. Hoffmann TC, Glasziou PP, Boutron I, Milne R, Perera R, Moher D, et al. Better reporting of interventions: template for intervention description and replication (TIDieR) checklist and guide. BMJ 2014;348:g1687. https://doi.org/10.1136/bmj.g1687

69. Kulkarni R, Gibson J, Brownson P, Thomas M, Rangan A, Carr AJ, Rees JL. Subacromial shoulder pain. Shoulder Elbow 2015;7:135-43. https://doi.org/10.1177/1758573215576456

70. Shawe-Taylor M, Rigby J. Cognitive behaviour therapy: its evolution and basic principles. J R Soc Promot Health 1999;119:244-6. https://doi.org/10.1177/146642409911900408

71. Moseley GL, Butler DS. Fifteen years of explaining pain: the past, present, and future. J Pain 2015;16:807-13. https://doi.org/10.1016/j.jpain.2015.05.005

72. Williams MA, Williamson EM, Heine PJ, Nichols V, Glover MJ, Dritsaki M, et al. Strengthening And stretching for Rheumatoid Arthritis of the Hand (SARAH). A randomised controlled trial and economic evaluation. Health Technol Assess 2015;19(19). https://doi.org/10.3310/hta19190

73. Borg GA. Psychophysical bases of perceived exertion. Med Sci Sports Exerc 1982;14:377-81. https://doi.org/10.1249/00005768-198205000-00012

74. McGuigan MR, Foster C. A new approach to monitoring resistance training. Strength Cond J 2004;26:42-7. https://doi.org/10.1519/00126548-200412000-00008

75. McClure P, Balaicuis J, Heiland D, Broersma ME, Thorndike CK, Wood A. A randomized controlled comparison of stretching procedures for posterior shoulder tightness. J Orthop Sports Phys Ther 2007;37:108-14. https://doi.org/10.2519/jospt.2007.2337

76. Hill AM, McPhail S, Hoffmann T, Hill K, Oliver D, Beer C, et al. A randomized trial comparing digital video disc with written delivery of falls prevention education for older patients in hospital. J Am Geriatr Soc 2009;57:1458-63. https://doi.org/10.1111/j.1532-5415.2009.02346.x

77. Michie S, Richardson M, Johnston M, Abraham C, Francis J, Hardeman W, et al. The behavior change technique taxonomy ( $\mathrm{v} 1$ ) of 93 hierarchically clustered techniques: building an international consensus for the reporting of behavior change interventions. Ann Behav Med 2013;46:81-95. https://doi.org/10.1007/s12160-013-9486-6 
78. Peek K, Sanson-Fisher R, Mackenzie L, Carey M. Interventions to aid patient adherence to physiotherapist prescribed self-management strategies: a systematic review. Physiotherapy 2016;102:127-35. https://doi.org/10.1016/j.physio.2015.10.003

79. Michie SRN, Fussell A, Hardeman W, Johnston M, Newman S, Yardley L. Improving Health: Changing Behaviour, NHS Health Trainer Handbook 200818 November 2016. URL: https://uwe-repository. worktribe.com/output/1011599/improving-health-changing-behaviour-nhs-health-trainer-handbook (accessed 21 June 2021).

80. Stone AA, Shiffman S, Schwartz JE, Broderick JE, Hufford MR. Patient compliance with paper and electronic diaries. Control Clin Trials 2003;24:182-99. https://doi.org/10.1016/S0197-2456 (02)00320-3

81. Abraham C, Michie S. A taxonomy of behavior change techniques used in interventions. Health Psychol 2008;27:379-87. https://doi.org/10.1037/0278-6133.27.3.379

82. Hopewell S, Keene DJ, Marian IR, Dritsaki M, Heine O, Cureton L, et al. Progressive exercise compared with best practice advice, with or without corticosteroid injection, for the treatment of patients with rotator cuff disorders (GRASP): a multicentre, pragmatic, $2 \times 2$ factorial, randomised controlled trial [published online ahead of print 12 July 2021]. Lancet 2021. https://doi.org/10.1016/S0140-6736(21)00846-1

83. McHorney CA, Tarlov AR. Individual-patient monitoring in clinical practice: are available health status surveys adequate? Qual Life Res 1995;4:293-307. https://doi.org/10.1007/BF01593882

84. Ridyard $\mathrm{CH}$, Hughes DA. Methods for the collection of resource use data within clinical trials: a systematic review of studies funded by the UK Health Technology Assessment program. Value Health 2010;13:867-72. https://doi.org/10.1111/j.1524-4733.2010.00788.x

85. Dritsaki M, Dakin H, Hopewell S, Lamb SE. Clinical and Cost Effectiveness of Progressive Exercise Compared to Best Practice Advice, With or Without Corticosteroid Injection, for the Treatment of Rotator Cuff Disorders: A $2 \times 2$ Factorial Randomised Controlled Trial - Health Economics Analysis Plan. URL: https://ora.ox.ac.uk/objects/uuid:e33a9c3c-2b7f-4c5a-969f-d31729652c9c (accessed 21 October 2020).

86. Petrou S, Gray A. Economic evaluation alongside randomised controlled trials: design, conduct, analysis, and reporting. BMJ 2011;342:d1548. https://doi.org/10.1136/bmj.d1548

87. Hughes D, Charles J, Dawoud D, Edwards RT, Holmes E, Jones C, et al. Conducting economic evaluations alongside randomised trials: current methodological issues and novel approaches. PharmacoEconomics 2016;34:447-61. https://doi.org/10.1007/s40273-015-0371-y

88. Dakin H, Gray A. Economic evaluation of factorial randomised controlled trials: challenges, methods and recommendations. Stat Med 2017;36:2814-30. https://doi.org/10.1002/sim.7322

89. Curtis L, Burns A. Unit Costs of Health and Social Care 2019. Canterbury: Personal Social Services Research Unit, University of Canterbury; 2019. www.pssru.ac.uk/project-pages/ unit-costs/unit-costs-2019/ (accessed 16 September 2020).

90. Withrington $\mathrm{RH}$, Girgis FL, Seifert $\mathrm{MH}$. A placebo-controlled trial of steroid injections in the treatment of supraspinatus tendonitis. Scand J Rheumatol 1985;14:76-8. https://doi.org/ 10.3109/03009748509102022

91. Searle E, Nicolson P, Hopewell S. Comparative Effectiveness of Subacromial Corticosteroid Injection Versus Other Non-Surgical Interventions for Improving Pain and Function in People with a Rotator Cuff Disorder: A Systematic Review and Meta-Analysis. MSc thesis. Paris: Descartes University; 2020. 
92. NHS Improvement. NHS Reference Costs 2017. URL: https://webarchive.nationalarchives.gov. uk/20200501111106/https://improvement.nhs.uk/resources/reference-costs/ (accessed 25 May 2021).

93. Karjalainen TV, Jain NB, Page CM, Lähdeoja TA, Johnston RV, Salamh P, et al. Subacromial decompression surgery for rotator cuff disease. Cochrane Database Syst Rev 2019;1:CD005619. https://doi.org/10.1002/14651858.CD005619.pub3

94. Herdman M, Gudex C, Lloyd A, Janssen M, Kind P, Parkin D, et al. Development and preliminary testing of the new five-level version of EQ-5D (EQ-5D-5L). Qual Life Res 2011;20:1727-36. https://doi.org/10.1007/s11136-011-9903-x

95. Rabin R, Oemar M, Oppe M, Janssen B, Herdman M. EQ-5D-5L User Guide. Basic Information on How to Use the EQ-5D-5L Instrument. Rotterdam: EuroQol Group; 2011.

96. van Hout B, Janssen MF, Feng YS, Kohlmann T, Busschbach J, Golicki D, et al. Interim scoring for the EQ-5D-5L: mapping the EQ-5D-5L to EQ-5D-3L value sets. Value Health 2012;15:708-15. https://doi.org/10.1016/j.jval.2012.02.008

97. Dolan P. Modeling valuations for EuroQol health states. Med Care 1997;35:1095-108. https://doi.org/10.1097/00005650-199711000-00002

98. Drummond MF, Sculpher MJ, Torrance GW, O'Brien BJ, Stoddart GL. Methods for the Economic Evaluation of Health Care Programmes. 3rd edn. New York, NY: Oxford University Press; 2005.

99. White IR, Royston P, Wood AM. Multiple imputation using chained equations: issues and guidance for practice. Stat Med 2011;30:377-99. https://doi.org/10.1002/sim.4067

100. National Institute for Health and Care Excellence. Judging Whether Public Health Interventions Offer Value for Money. URL: www.nice.org.uk/advice/lgb10/chapter/judging-the-cost-effectivenessof-public-health-activities (accessed 16 September 2020).

101. Manca A, Hawkins N, Sculpher MJ. Estimating mean QALYs in trial-based cost-effectiveness analysis: the importance of controlling for baseline utility. Health Econ 2005;14:487-96. https://doi.org/10.1002/hec.944

102. Schomaker M, Heumann C. Bootstrap inference when using multiple imputation. Stat Med 2018;37:2252-66. https://doi.org/10.1002/sim.7654

103. Dakin H, Farmer A, Gray A, Holman RR. Economic evaluation of factorial trials: cost-utility analysis of the atorvastatin in factorial with omega EE90 risk reduction in diabetes $2 \times 2 \times 2$ factorial trial of atorvastatin, omega-3 fish oil, and action planning. Econ Eval 2020;23:134-08. https://doi.org/10.1016/j.jval.2020.05.018

104. Lubsen J, Pocock SJ. Factorial trials in cardiology: pros and cons. Eur Heart J 1994;15:585-8. https://doi.org/10.1093/oxfordjournals.eurheartj.a060552

105. Sadatsafavi M, Marra C, Aaron S, Bryan S. Incorporating external evidence in trial-based cost-effectiveness analyses: the use of resampling methods. Trials 2014;15:201. https://doi.org/ 10.1186/1745-6215-15-201

106. White JJ, Titchener AG, Fakis A, Tambe AA, Hubbard RB, Clark DI. An epidemiological study of rotator cuff pathology using The Health Improvement Network database. Bone Joint $J$ 2014;96:350-3. https://doi.org/10.1302/0301-620X.96B3.32336

107. Office for National Statistics. Ethnicity. URL: www.ons.gov.uk/peoplepopulationandcommunity/ culturalidentity/ethnicity (accessed 21 June 2021).

108. Gumina S, Candela V, Passaretti D, Latino G, Venditto T, Mariani L, Santilli V. The association between body fat and rotator cuff tear: the influence on rotator cuff tear sizes. J Shoulder Elbow Surg 2014;23:1669-74. https://doi.org/10.1016/j.jse.2014.03.016 
109. Warrender WJ, Brown OL, Abboud JA. Outcomes of arthroscopic rotator cuff repairs in obese patients. J Shoulder Elbow Surg 2011;20:961-7. https://doi.org/10.1016/j.jse.2010.11.006

110. Krischak G, Gebhard F, Reichel H, Friemert B, Schneider F, Fisser C, et al. A prospective randomized controlled trial comparing occupational therapy with home-based exercises in conservative treatment of rotator cuff tears. J Shoulder Elbow Surg 2013;22:1173-9. https://doi.org/10.1016/j.jse.2013.01.008

111. Granviken F, Vasseljen $O$. Home exercises and supervised exercises are similarly effective for people with subacromial impingement: a randomised trial. J Physiother 2015;61:135-41. https://doi.org/10.1016/j.jphys.2015.05.014

112. Asensio-García MDR, Bernabeú-Casas RC, Palazón-Bru A, Tomás-Rodríguez MI, Nouni-García R. Effectiveness of a group physiotherapy intervention in nontraumatic, inoperable painful shoulder: a randomized clinical trial. Am J Phys Med Rehabil 2018;97:110-15. https://doi.org/10.1097/ PHM.0000000000000817

113. Contreras J, Liendo R, Díaz C, Díaz M, Osorio M, Guzmán R, et al. [Effectiveness of a self-administered rehabilitation program for shoulder pain syndrome in primary health care.] Rev Med Chil 2018;146:959-67. https://doi.org/10.4067/s0034-98872018000900959

114. Türkmen E, Analay Akbaba Y, Altun S. Effectiveness of video-based rehabilitation program on pain, functionality, and quality of life in the treatment of rotator cuff tears: a randomized controlled trial. J Hand Ther 2020;33:288-95. https://doi.org/10.1016/j.jht.2019.08.004

115. Roddy E, Ogollah RO, Oppong R, Zwierska I, Datta P, Hall A, et al. Optimising outcomes of exercise and corticosteroid injection in patients with subacromial pain (impingement) syndrome: a factorial randomised trial. Br J Sport Med 2021;55:262-71. https://doi.org/ 10.1136/bjsports-2019-101268

116. Gialanella B, Prometti P. Effects of corticosteroids injection in rotator cuff tears. Pain Med 2011;12:1559-65. https://doi.org/10.1111/j.1526-4637.2011.01238.x

117. Adebajo AO, Nash P, Hazleman BL. A prospective double blind dummy placebo controlled study comparing triamcinolone hexacetonide injection with oral diclofenac $50 \mathrm{mg}$ TDS in patients with rotator cuff tendinitis. J Rheumatol 1990;17:1207-10.

118. Alvarez CM, Litchfield R, Jackowski D, Griffin S, Kirkley A. A prospective, double-blind, randomized clinical trial comparing subacromial injection of betamethasone and xylocaine to xylocaine alone in chronic rotator cuff tendinosis. Am J Sports Med 2005;33:255-62. https://doi.org/10.1177/0363546504267345

119. Alvarez-Nemegyei J, Bassol-Perea A, Rosado Pasos J. [Efficacy of the local injection of methylprednisolone acetate in the subacromial impingement syndrome. A randomized, doubleblind trial.] Reumatol Clin 2008;4:49-54. https://doi.org/10.1016/S1699-258X(08)71799-6

120. Blair B, Rokito AS, Cuomo F, Jarolem K, Zuckerman JD. Efficacy of injections of corticosteroids for subacromial impingement syndrome. J Bone Joint Surg Am 1996;78:1685-9. https://doi.org/ 10.2106/00004623-199611000-00007

121. Hong JY, Yoon SH, Moon DJ, Kwack KS, Joen B, Lee HY. Comparison of high- and low-dose corticosteroid in subacromial injection for periarticular shoulder disorder: a randomized, triple-blind, placebo-controlled trial. Arch Phys Med Rehabil 2011;92:1951-60. https://doi.org/ 10.1016/j.apmr.2011.06.033

122. Penning $\mathrm{LI}$, de Bie RA, Walenkamp GH. The effectiveness of injections of hyaluronic acid or corticosteroid in patients with subacromial impingement: a three-arm randomised controlled trial. J Bone Joint Surg Br 2012;94:1246-52. https://doi.org/10.1302/0301-620X.94B9.28750 
123. Petri M, Dobrow R, Neiman R, Whiting-O'Keefe Q, Seaman WE. Randomized, double-blind, placebo-controlled study of the treatment of the painful shoulder. Arthritis Rheum 1987;30:1040-5. https://doi.org/10.1002/art.1780300911

124. Vecchio PC, Hazleman BL, King RH. A double-blind trial comparing subacromial methylprednisolone and lignocaine in acute rotator cuff tendinitis. $\mathrm{Br} J$ Rheumatol 1993;32:743-5. https://doi.org/10.1093/rheumatology/32.8.743

125. Beard DJ, Rees JL, Cook JA, Rombach I, Cooper C, Merritt N, et al. Arthroscopic subacromial decompression for subacromial shoulder pain (CSAW): a multicentre, pragmatic, parallel group, placebo-controlled, three-group, randomised surgical trial. Lancet 2018;391:329-38. https://doi.org/10.1016/S0140-6736(17)32457-1

126. Dean BJ, Carr AJ. The effects of glucocorticoid on tendon and tendon derived cells. Adv Exp Med Biol 2016;920:239-46. https://doi.org/10.1007/978-3-319-33943-6_23

127. NHS. NHS Electronic Drug Tariff. 2020. URL: www.drugtariff.nhsbsa.nhs.uk/\#/00770298-DC/ DC00770293/Home (accessed 25 May 2021).

128. NHS Improvement. NHS Reference Costs 2019. URL: www.england.nhs.uk/national-cost-collection/ (accessed 25 May 2021).

129. Curtis L, Burns A. Unit Costs of Health and Social Care 2010. Canterbury: Personal Social Services Research Unit, University of Kent; 2010. URL: www.pssru.ac.uk/project-pages/ unit-costs/unit-costs-2010/ (accessed 25 May 2021).

130. Curtis L, Burns A. Unit Costs of Health and Social Care 2015. Canterbury: Personal Social Services Research Unit, University of Kent; 2015. URL: www.pssru.ac.uk/project-pages/unitcosts/unit-costs-2015/ (accessed 25 May 2021).

131. Murgia M. NHS to trial artificial intelligence app in place of 111 helpline. Financial Times, 4 January 2017.

132. WhatClinic. Orthopaedic Clinics UK. URL: www.whatclinic.com/orthopaedic/uk (accessed 25 May 2021).

133. The Physiotherapy Centre. Prices. 2020. URL: www.thephysiotherapycentre.org.uk/prices (accessed 23 June 2021).

134. Private Healthcare UK. How Much Does a Private Ultrasound Cost in the UK? URL: www. privatehealth.co.uk/conditions-and-treatments/ultrasound/costs/ (accessed 25 May 2021).

135. Office for National Statistics. Employee Earnings in the UK: 2019. URL: www.ons.gov.uk/ releases/employeeearningsintheuk2019 (accessed 23 June 2021).

136. Department of Health and Social Care (DHSC). Social Care - Charging for Care and Support. London: DHSC; 2017. 



\section{Appendix 1 Chapter 1 appendix}

\section{Diagnosis of Shoulder problems in Primary Care: Guidelines on treatment and referral}



FIGURE 13 The BESS diagnostic algorithm. ${ }^{4}$ Reproduced with permission (Professor Jonathan L Rees, Nuffield Orthopaedic Centre, 2021, personal communication). 



\section{Appendix 2 Chapter 2 appendix}

\begin{tabular}{|c|c|c|c|c|}
\hline $\begin{array}{l}\text { Amendment } \\
\text { number }\end{array}$ & $\begin{array}{l}\text { Protocol } \\
\text { version } \\
\text { number }\end{array}$ & Date issued & $\begin{array}{l}\text { Author(s) of } \\
\text { changes }\end{array}$ & Details of changes made \\
\hline 1 & Version 2.0 & 20 March 2017 & Sally Hopewell & $\begin{array}{l}\text { Clarification of eligibility criteria to include } \\
\text { those predominantly seeking treatment for } \\
\text { one shoulder } \\
\text { Clarification of timelines for injection and } \\
\text { physiotherapy referral } \\
\text { Minor clarifications on physiotherapy } \\
\text { intervention content, including revision } \\
\text { of Figure } 2 \\
\text { Correction to month of recruitment } \\
\text { Addition of PROMPTS (personalised text } \\
\text { message versus standard text message } \\
\text { prompts for increasing response to postal } \\
\text { questionnaires) substudy }\end{array}$ \\
\hline 4 & Version 3.0 & 13 September 2017 & Sally Hopewell & $\begin{array}{l}\text { Minor clarification regarding methods of data } \\
\text { collection and management } \\
\text { Minor change of wording regarding injection } \\
\text { delivery }\end{array}$ \\
\hline 12 & Version 4.0 & 14 May 2018 & Sally Hopewell & $\begin{array}{l}\text { Addition of monetary incentive at } 12 \text {-month } \\
\text { follow-up }\end{array}$ \\
\hline 16 & Version 5.0 & 2 January 2019 & Sally Hopewell & $\begin{array}{l}\text { Addition of text reminder at } 12 \text { months } \\
\text { Change to wording regarding duration of } \\
\text { recruitment }\end{array}$ \\
\hline
\end{tabular}




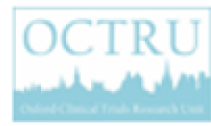

\section{GRASP Injection Referral Form and Treatment Log}

PART 1: GRASP Injection Referral Form (to be completed by the research clinician)

Appointments should be scheduled so that the participant receives their injection within 10 days of randomisation.

DARTICIPANT ID:
\begin{tabular}{|l|l|l|l|l|l|l|l|l|l|l|l|l|}
\hline G & R \\
\hline
\end{tabular}

\section{PARTICIPANT DETAILS}

Full name:

Date participant randomised:

Date of birth:

NHS Number:

Participant address:

Phone 1:

Phone 2:

Email:

REFERREO BY (name):

Position:

PART 2: GRASP Injection Treatment Log (to be completed by the injecting therapist).

The therapist should follow usual local procedure for their Trust when administering the corticosteroid injection.
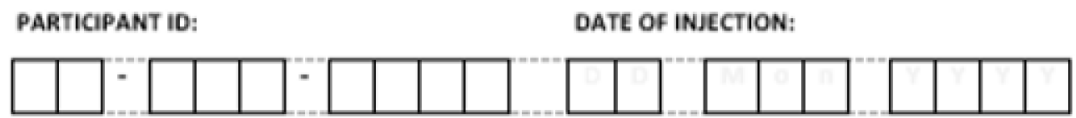

Name of injecting therapist:

1. Corticosteroid injected (tick): $\square$ Methylprednisolone acetate

口Triamcinolone acetonide

2. Dose of corticosteroid injected ${ }^{*}(\mathrm{mg})$ :

3. Choice of local anaesthetic injected (tick): $\square 1 \%$ lidocaine $\quad \square 0.5 \%$ Bupivacaine hydrochloride

4. volume of local anaesthetic injected * $(\mathrm{m} / \mathrm{s})$ :

5. Corticosteroid and local anaesthetic given as (tick): $\square$ combined injection $\square$ separate injection

6. Which shoulder is injected (tick): $\square$ Left $\square$ Right

7. Site of injection (tick): $\square$ Anterior subacromial space $\square$ Posterior subacromial space

$\square$ other (specify):

8. Participant given local injection advice leaflet (tick): $\square$

9. Participant given advice on what to do if adverse event occurs (tick): $\square$

If injection not given pleose contact GRASP Trial Office as soon as possible on: 01865737432.

Injection not given (tick): $\square$ Reason injection not given:

"Choice of corticosteroid injection can be either Methyiprednisane acetote (up to $40 \mathrm{mg}$ ) or Triamcinolone acetonide (up to 40mg). Chaice of local anaesthetic can be either $1 . \mathrm{K}$ lidocaine (up to $5 \mathrm{~m} /$ ) or $0.5 \%$ Bupivacaine hydrochloride (up to $10 \mathrm{~m}$ ) ONE FULL COPY RETAINED IN GRASP INVESTIGATOR SITE FILE, ONE FULL COPY IN PATIENT NOTES, PART 2 ONLY TO BE SENT TO THE GRASP TRIAL OFFICE - REDACT AS NECESSARY

FIGURE 14 Injection referral form and treatment log. OCTRU logo reproduced with permission (Vicki Barber, Oxford Clinical Trials Research Unit, 2021, personal communication). 


\section{Appendix 3 Chapter 3 appendix}

TABLE 26 Categories of exercise considered by the delegates of the GRASP trial intervention development meeting

$\begin{array}{ll}\text { Exercise category } & \frac{\text { Included in intervention }}{\text { Best-practice advice Progressive exercise }}\end{array}$

Pectoralis major and anterior capsule stretch

Posterior shoulder/capsule stretch

Other shoulder stretches

Scapular stability exercises and 'setting' (isolated positional changes of the scapula prior to shoulder movement)

Scapular retraction

Scapular protractions

Scapular elevation/depression

Press-ups

External shoulder rotation (low challenge exercises)

External shoulder rotation (in shoulder elevation $<90^{\circ}$ )

External shoulder rotation (in shoulder elevation $\approx 90^{\circ}$ )

External shoulder rotation (in shoulder elevation $>90^{\circ}$ )

Internal shoulder rotation (low challenge exercises)

Internal shoulder rotation (in shoulder elevation $<90^{\circ}$ )

Internal shoulder rotation (in shoulder elevation $\approx 90^{\circ}$ )

Pendulum exercise

Assisted shoulder flexion

Active shoulder flexion (unresisted)

Shoulder extension

Shoulder adduction

Shoulder abduction

Resisted functional movement

Thoracic extension

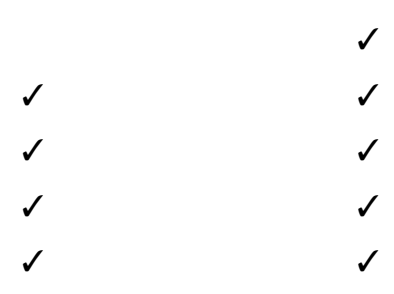

TABLE 27 Behavioural strategies to address barriers to exercise, highlighting common and discrete components to each exercise intervention

\section{Behaviour change}

\section{technique}

Education and persuasion

Training
Progressive exercise

Information on benefits of exercise provided face to face and in participant information booklet

Exercises demonstrated, instructions provided in written form, exercises practised within the session and at home independently, and exercises reviewed at each appointment and feedback provided

\section{Best-practice advice}

Information on benefits of exercise provided face to face and in participant information booklet

Exercises demonstrated, instructions provided in written and video form (web and/or DVD), and exercises practised within the session and at home independently 
TABLE 27 Behavioural strategies to address barriers to exercise, highlighting common and discrete components to each exercise intervention (continued)

Behaviour change technique

Graded tasks

Equipment provision to enable exercise

Problem-solving

Action planning

Goal-setting

Review goals

Confidence rule

Feedback on exercise performance from physiotherapist

Therapist feedback on outcome of exercise

Self-monitoring of exercise

Behavioural contract

\section{Progressive exercise}

Focus on starting with a manageable amount of exercise (up to three exercises). Use of modified Borg scale to set resistance exercise [initially at lower levels and then increase difficulty, volume and load (see Figure 3)]

Resistance band issued if appropriate. Advice on what can be used for hand weights

Over the sessions, therapists facilitate participant problem-solving and encourage the participant to lead the decision-making about progression/regression of programme. Barriers to exercise (e.g. belief or practicalities) are reviewed and ways to overcome these are discussed. An exercise plan is agreed collaboratively

An action planner document is to be completed (i.e. where and when exercises will be carried out), including contingency plans, prompts/cues for when to do the exercises, a plan of what to do if pain increases and information on when and where the exercise diary will be completed. See also Behavioural contract and Confidence ruler

Agree a long- and a short-term goal (which are specific, measurable, achievable, relevant and timed) and reinforce link between goal and exercise plan

Goals reviewed and adjusted at each session

Use the confidence ruler (from 0 to 10 ) to assess participant's confidence in undertaking agreed exercise plan. If the participant selects $\leq 7$, discuss reasons and adapt programme (see Graded tasks) or address barriers (see Problem-solving)

Within and between sessions, review action planners and diaries at each session, and review discrepancies in plan and performance

Progress in physical performance and symptoms highlighted after reassessment at each session

Exercise diary to complete each day

Therapist and participant sign action planner document. Participant commits to undertaking exercises and bringing documents to be reviewed at next appointment. Therapist to review exercise diary

Simple advice on managing low mood in the information booklet. Provide reassurance about condition and capacity to exercise

\section{Best-practice advice}

Focus on starting with a manageable amount of exercise (one or two exercises). Participants to increase volume and load according to set progression advice

All participants issued resistance band to enable exercise performance. Advice on what can be used for hand weights

Therapists encourage participant problemsolving within the session. A starting point on exercise ladder is agreed collaboratively

An action planner document is to completed (i.e. where and when exercises will be carried out), including contingency plans, prompts/cues for when to do the exercises, a plan of what to do if pain increases and information on when and where the exercise diary will be completed

Agree a long-term goal (which is specific measurable, achievable, relevant and timed) and reinforce link between goal and exercise plan

Not applicable

Not applicable

In session only

Not applicable

Same as Progressive exercise

Not applicable

Same as Progressive exercise 
My confidence: On a scale of 0 to 10 , how confident am I that I can carry out my exercise programme? (circle)

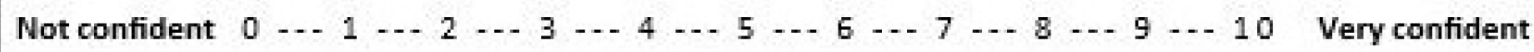

My long term goal is: (for example to retum to gardening or work)

My short term goal is: (for example be to be able to lift a saucepan or drive)

It is important to think about when and how you will do your exercises.

When it is (time or other cue) I will do my exercises. If I cannot do them at this time I will do them:

I will remind myself to do my exercises by:

If my pain increases with the exercises then I will:

Exercise diarya It is important to measure and record your progress, so that you can see when you are succeeding, as well as to work out what you can change if your plan is not working.

When will I record it?

Where will I complete my diary?

Patient: I will do my exercises, record my progress (Exercise Diary) and bring my exercise diary to the next meeting.

Date:

Signature:
Physiotherapist: I will discuss your exercise diary with you and how you got on when you bring it back at the next meeting.

Date:

Signature:

At your next appointment. This form will be reviewed at your next physiotherapy session along with your exercise diary. If any parts have changed or needs to change, a new planner can be completed. 


\section{GRASP Progressive Exercise}

\section{Diary}

Participant Name:

Date started:

Please add the number or times you repeat an exercise and the number of sets into the boxes for each exercise each day.

If you have not done your exercises as it is a planned rest day mark the box with a cross ( $\mathrm{x}$ ), if you forgot, mark it with a zero (0).

\begin{tabular}{|c|c|c|c|c|c|c|}
\hline \multirow[t]{2}{*}{ Day } & \multicolumn{2}{|l|}{ Exercise: } & \multicolumn{2}{|l|}{ Exercise: } & \multicolumn{2}{|l|}{ Exercise: } \\
\hline & Repetitions & Sets & Repetitions & Sets & Repetitions & Sets \\
\hline 1 & & & & & & \\
\hline 2 & & & & & & \\
\hline 3 & & & & & & \\
\hline 4 & & & & & & \\
\hline 5 & & & & & & \\
\hline 6 & & & & & & \\
\hline 7 & & & & & & \\
\hline 8 & & & & & & \\
\hline 9 & & & & & & \\
\hline 10 & & & & & & \\
\hline 11 & & & & & & \\
\hline 12 & & & & & & \\
\hline 13 & & & & & & \\
\hline 14 & & & & & & \\
\hline
\end{tabular}

FIGURE 16 Progressive exercise intervention: exercise diary. OCTRU logo reproduced with permission (Vicki Barber, Oxford Clinical Trials Research Unit, 2021, personal communication). 
1a Shoulder flexion in sitting, supported by table
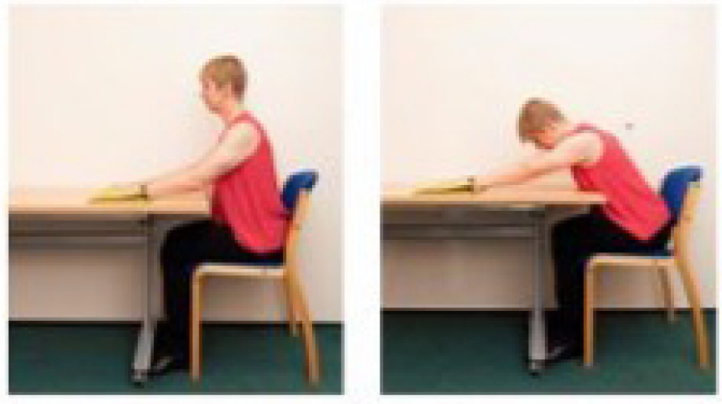

1c Shoulder flexion in standing, supported by table
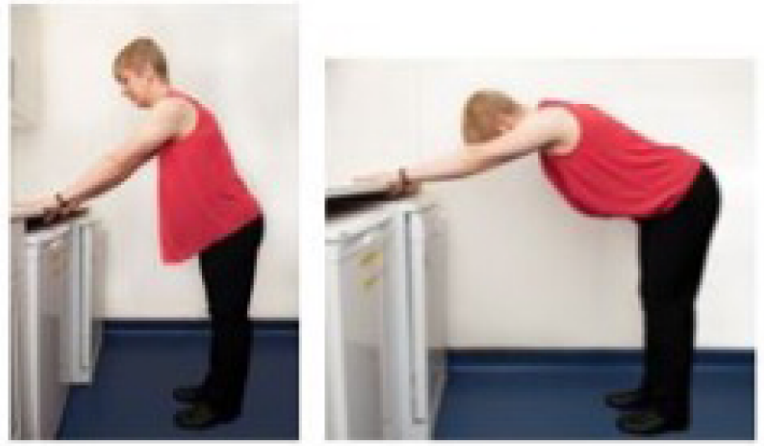

$1 b$ Shoulder abduction in sitting, supported by table

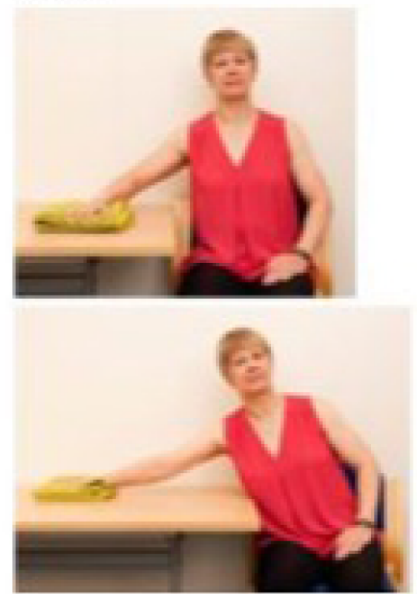

$1 \mathrm{~d}$ Shoulder abduction in standing, supported by table
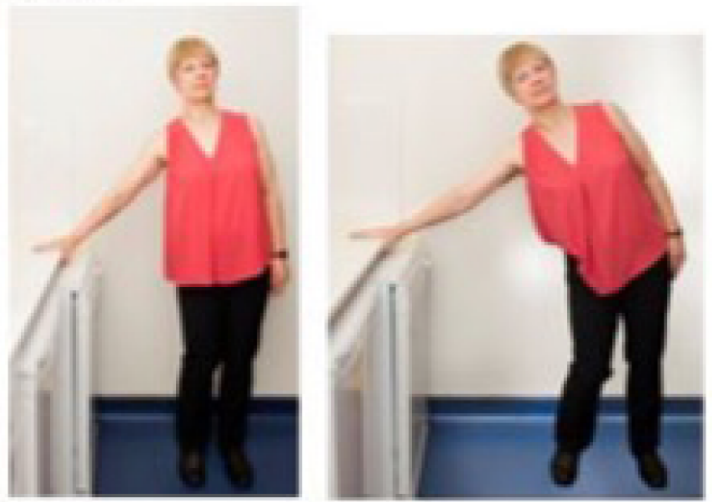

If Assisted shoulder flexion, using wall
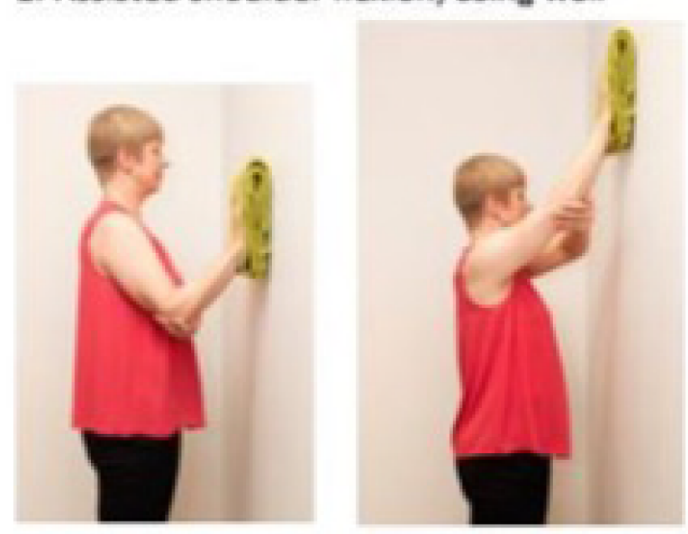

1e Shoulder flexion in supine

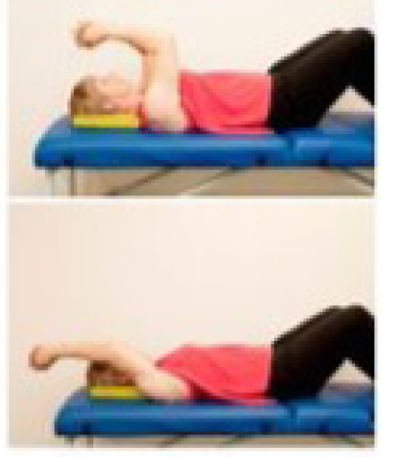

FIGURE 17 Progressive exercise intervention: level 1 simple shoulder movement exercises. Exercises can be modified in a number of ways: (1) the range of motion through which an exercise is performed may be increased or decreased; and (2) the position may be modified, for example some exercises may be carried out in lying, sitting or standing positions to accommodate the patient's comfort and preferences. 
2a Isometric external rotation

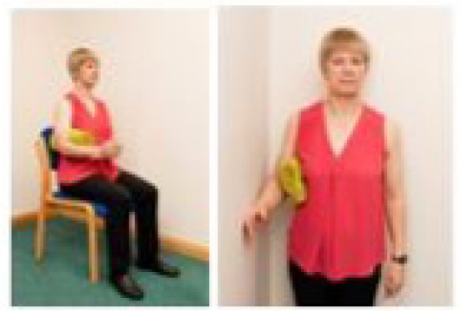

2c External rotation with band

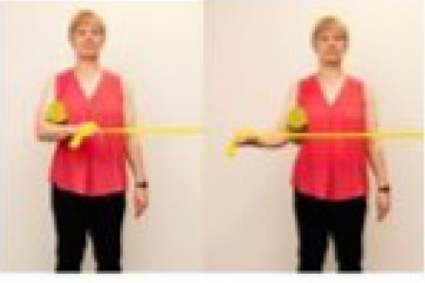

2e Shoulder flexion using wall

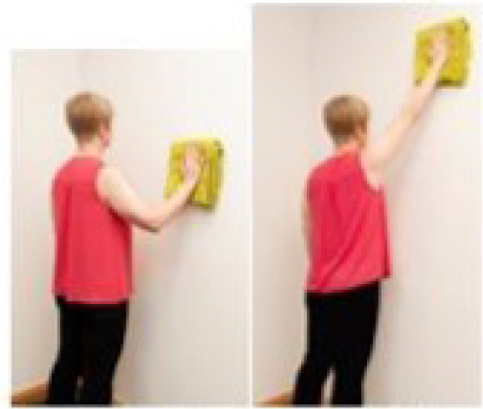

$\mathrm{2g}$ External rotation with band, arm elevated and supported


2i Arm elevation with resisted external rotation

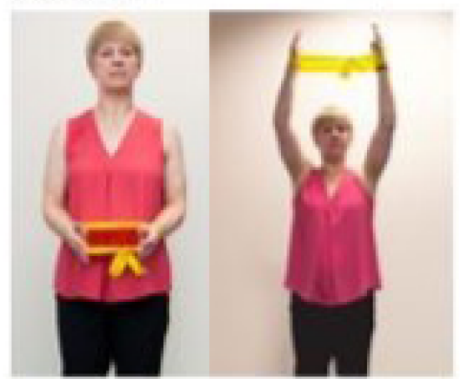

2b Isometric abduction

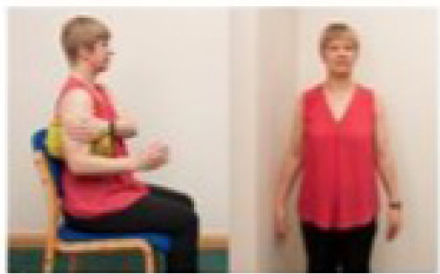

$2 \mathrm{~d}$ Abduction with a band

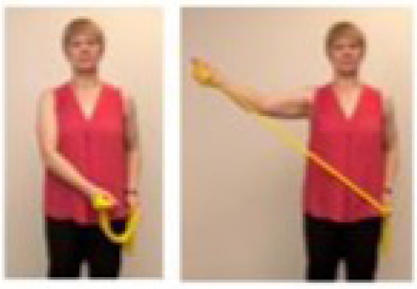

2 Arm raise using a weight or resistance band

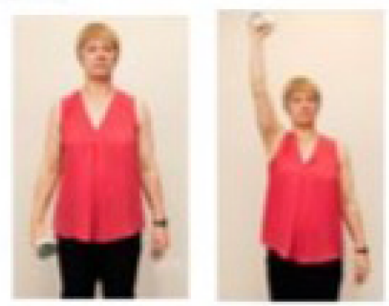

2h External rotation with band, arm elevated and unsupported
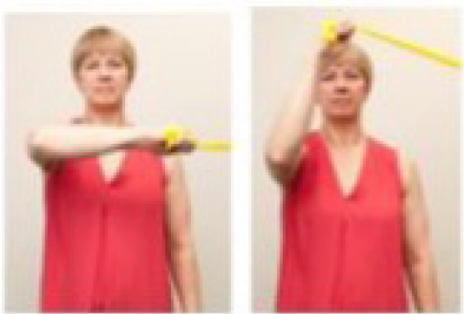

\section{2j Abduction and elevation with band}

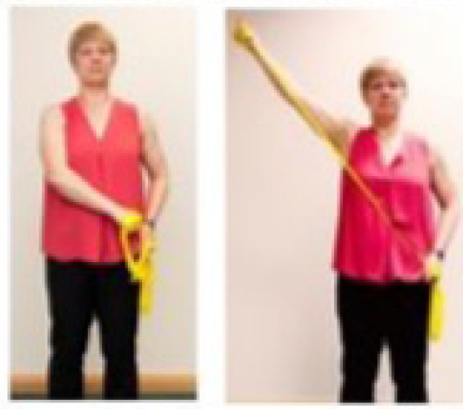

FIGURE 18 Progressive exercise intervention: level 2 essential resistance exercises. Exercises can be modified in a number of ways: (1): the range of motion through which an exercise is performed may be increased or decreased; and (2) the position may be modified, for example some exercises may be carried out in lying, sitting or standing positions to accommodate the patient's comfort and preferences. 

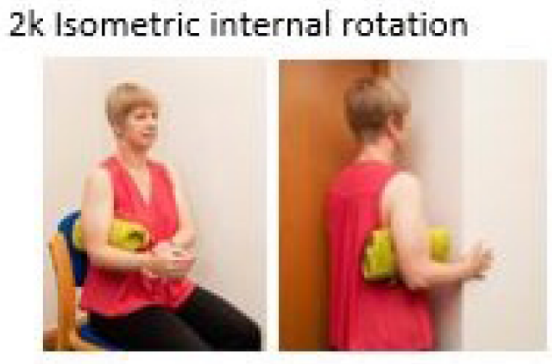

$2 \mathrm{~m}$ Internal rotation with band, arm elevated (supported)

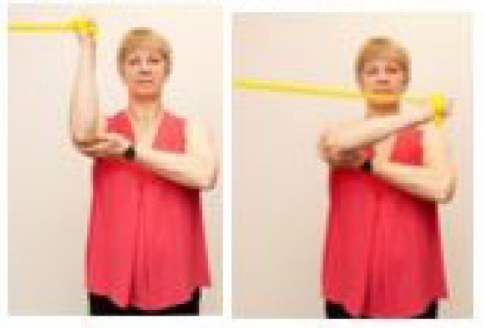

$2 p$ Prone shoulder lift

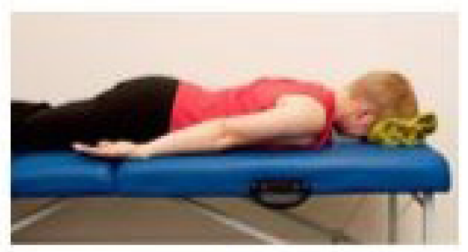

2s 4-point kneeling, arm elevation

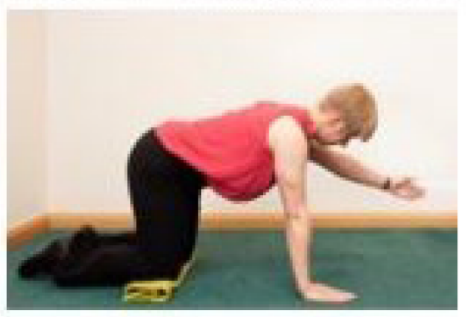

2) Internal rotation with band

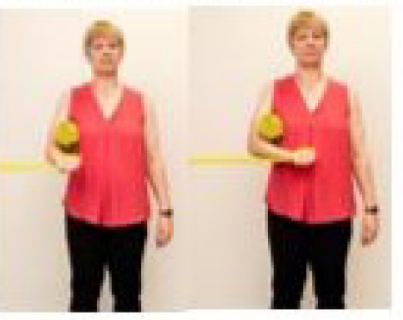

$2 n$ Internal rotation with band, arm elevated

(unsupported)

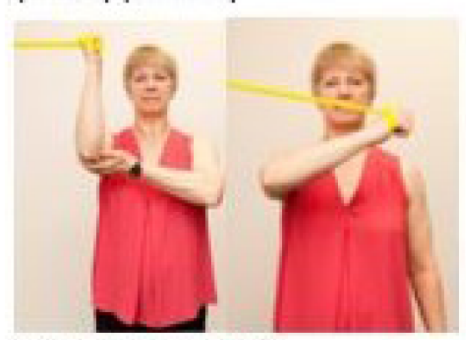

2q Prone arm lift, elbow flexed

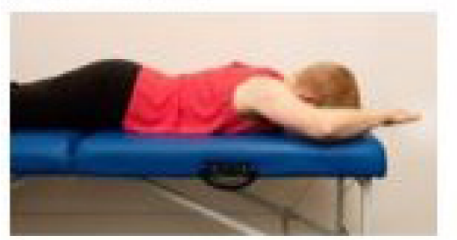

2r Prone arm lift, elbow extended

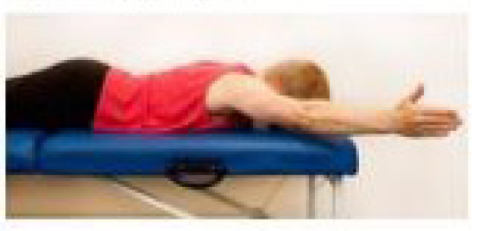

2u Full press-up

2t Press-ups on knees



FIGURE 19 Progressive exercise intervention: level 2 optional resistance exercises. Exercises can be modified in a number of ways: (1): the range of motion through which an exercise is performed may be increased or decreased; and (2) the position may be modified, for example some exercises may be carried out in lying, sitting or standing positions to accommodate the patient's comfort and preferences. 
S1 Sleeper stretch

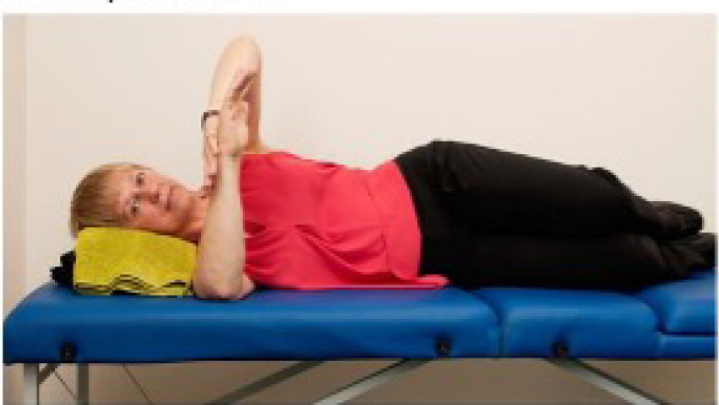

S2 Cross body stretch

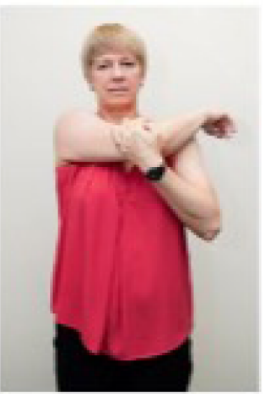

FIGURE 20 Progressive exercise intervention: level 2 optional resistance exercises. To be used selectively and only for younger adults engaged in throwing or other overhead athletic or physical activities ${ }^{75}$ who have posterior capsule tightness.

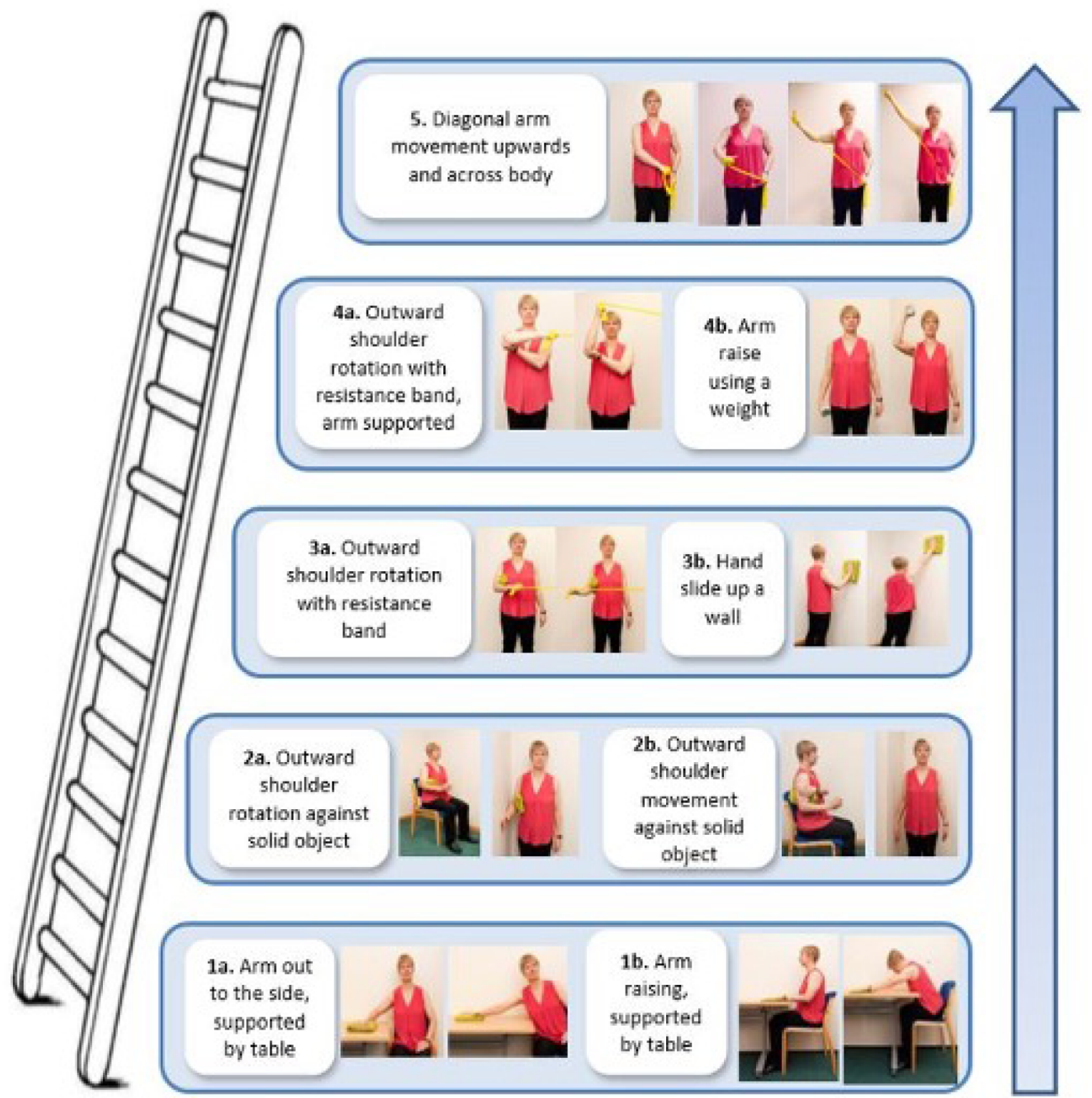

FIGURE 21 Best-practice advice intervention: exercise progression ladder. 
Participant Name:

Please add the number or times you repeat an exercise and the number of sets into the boxes for each exercise each day.

If you have not done your exercises as it is a planned rest day ( 2 days out of 7 ) mark the box with a cross $(x)$ if you forgot, mark it with a zero (0).

\begin{tabular}{|c|c|c|c|c|}
\hline \multirow[t]{2}{*}{ Day } & \multicolumn{2}{|l|}{ Exercise: } & \multicolumn{2}{|l|}{ Exercise: } \\
\hline & Repetitions & Sets & Repetitions & Sets \\
\hline \multicolumn{5}{|l|}{1} \\
\hline \multicolumn{5}{|l|}{2} \\
\hline \multicolumn{5}{|l|}{3} \\
\hline \multicolumn{5}{|l|}{4} \\
\hline \multicolumn{5}{|l|}{5} \\
\hline \multicolumn{5}{|l|}{6} \\
\hline \multicolumn{5}{|l|}{7} \\
\hline \multicolumn{5}{|l|}{8} \\
\hline \multicolumn{5}{|l|}{9} \\
\hline \multicolumn{5}{|l|}{10} \\
\hline \multicolumn{5}{|l|}{11} \\
\hline \multicolumn{5}{|l|}{12} \\
\hline \multicolumn{5}{|l|}{13} \\
\hline 14 & & & & \\
\hline
\end{tabular}

Date started.

FIGURE 22 Best-practice advice intervention: exercise diary. OCTRU logo reproduced with permission (Vicki Barber, Oxford Clinical Trials Research Unit, 2021, personal communication). 
Participant Name:

My long term goal is: (for example to retum to gardening or work)

It is important to think about when and how you will do your exercises.

When it is (time or other cue) I will do my

exercises. If I cannot do them at this time I will do them:

I will remind myself to do my exercises by:

If my pain increases with the exercises then I will:

Exercise diary: It is important to measure and record your progress, so that you can see when you are succeeding, as well as to work out what you can change if your plan is not working.

When will I record it?

Where will I complete my diary?

FIGURE 23 Best-practice advice intervention: action planner. OCTRU logo reproduced with permission (Vicki Barber, Oxford Clinical Trials Research Unit, 2021, personal communication). 


\section{Appendix 4 Chapter 4 appendix}

TABLE 28 Characteristics of participating NHS trusts

\begin{tabular}{|c|c|c|c|}
\hline Site & Start date & $\begin{array}{l}\text { Months of } \\
\text { recruitment }^{\mathrm{a}}\end{array}$ & $\begin{array}{l}\text { Number of } \\
\text { clinicians }^{\mathbf{b}}\end{array}$ \\
\hline University Hospitals of Derby and Burton NHS Foundation Trust & 20 February 2017 & 26 & 18 \\
\hline East Lancashire Hospitals NHS Trust & 2 March 2017 & 26 & 17 \\
\hline Gloucestershire Hospitals NHS Foundation Trust & 12 April 2017 & 24 & 43 \\
\hline Birmingham Community Healthcare NHS Trust & 13 April 2017 & 24 & 13 \\
\hline Sandwell and West Birmingham Hospitals NHS Trust & 19 May 2017 & 23 & 13 \\
\hline Buckinghamshire Musculoskeletal Integrated Care Service & 26 May 2017 & 23 & 3 \\
\hline Bedfordshire Hospitals NHS Foundation Trust & 8 June 2017 & 22 & 7 \\
\hline East Cheshire NHS Trust & 10 July 2017 & 21 & 8 \\
\hline Wirral University Teaching Hospital NHS Foundation Trust & 18 August 2017 & 20 & 6 \\
\hline Medway Community Healthcare & 10 August 2017 & 20 & 6 \\
\hline Bristol Community Health & 29 September 2017 & 19 & 4 \\
\hline $\begin{array}{l}\text { Doncaster and Bassetlaw Teaching Hospitals NHS } \\
\text { Foundation Trust }\end{array}$ & 27 November 2017 & 17 & 9 \\
\hline Somerset Partnership NHS Foundation Trust & 23 November 2017 & 17 & 13 \\
\hline Sherwood Forest Hospitals NHS Foundation Trust & 20 December 2017 & 16 & 4 \\
\hline Kent Community Health NHS Foundation Trust & 19 January 2018 & 15 & 10 \\
\hline Northern Devon Healthcare NHS Trust & 8 February 2018 & 14 & 17 \\
\hline Airedale NHS Foundation Trust & 20 February 2018 & 14 & 7 \\
\hline North West Boroughs Healthcare NHS Foundation Trust & 29 March 2018 & 13 & 10 \\
\hline Staffordshire and Stoke-on-Trent Partnership NHS Trust & 26 April 2018 & 12 & 6 \\
\hline Warrington and Halton Hospitals NHS Foundation Trust & 16 July 2018 & 9 & 9 \\
\hline Total & & 26 & 223 \\
\hline
\end{tabular}

a Recruitment ended 2 May 2019.

b The number of clinicians who delivered trial interventions.

TABLE 29 Characteristics of participating physiotherapists

\begin{tabular}{|c|c|c|c|c|c|c|}
\hline \multirow[b]{2}{*}{ Participating physiotherapist } & \multicolumn{6}{|c|}{ Agenda for Change grade, $n$ (\%) } \\
\hline & Band 5 & Band 6 & Band 7 & Band 8a & Other $^{a}$ & Total $^{\mathbf{b}}$ \\
\hline Injectors & $0(0)$ & $6(11)$ & $13(23)$ & $34(61)$ & $3(5)$ & $56(100)$ \\
\hline Best-practice advice & $17(20)$ & $38(46)$ & $22(27)$ & $6(7)$ & $0(0)$ & $83(100)$ \\
\hline Progressive exercise & $16(15)$ & $55(53)$ & $28(27)$ & $5(5)$ & $0(0)$ & $104(100)$ \\
\hline
\end{tabular}


TABLE 30 Reasons participants were ineligible or declined to participate

\begin{tabular}{|c|c|}
\hline Reason & $\begin{array}{l}\text { Total, } \\
n(\%)\end{array}$ \\
\hline Ineligible, $n$ & 1003 \\
\hline History of significant shoulder trauma (e.g. dislocation, fracture, full-thickness tear requiring surgery) & $94(9)$ \\
\hline Neurological disease affecting shoulder & $23(2)$ \\
\hline $\begin{array}{l}\text { Other shoulder disorder (e.g. inflammatory arthritis, frozen shoulder, glenohumeral joint instability) } \\
\text { or red flags consistent with BESS criteria }\end{array}$ & $434(43)$ \\
\hline Received corticosteroid injection or physiotherapy for shoulder pain in last 6 months & $167(17)$ \\
\hline Contraindications to corticosteroid injection & $31(3)$ \\
\hline Symptoms $>6$ months & $132(13)$ \\
\hline Did not speak English & $32(3)$ \\
\hline Other reason & $28(3)$ \\
\hline Other (neither declined nor ineligible) & $3(0.3)$ \\
\hline Unable to contact & $59(6)$ \\
\hline Declined, $n$ & 576 \\
\hline Not interested in taking part in research & $175(30)$ \\
\hline Does not want to be randomised & $39(7)$ \\
\hline Already has treatment preference for receiving injection & $38(7)$ \\
\hline Already has treatment preference for not receiving injection & $158(27)$ \\
\hline Does not want to be randomised to receive progressive-exercise intervention & $6(1)$ \\
\hline Does not want to be randomised to receive best-practice advice intervention & $8(1)$ \\
\hline Unable to attend treatment sessions & $71(12)$ \\
\hline Leaving the area & $9(2)$ \\
\hline Prefer not to say & $20(3)$ \\
\hline Other/no information & $52(9)$ \\
\hline
\end{tabular}




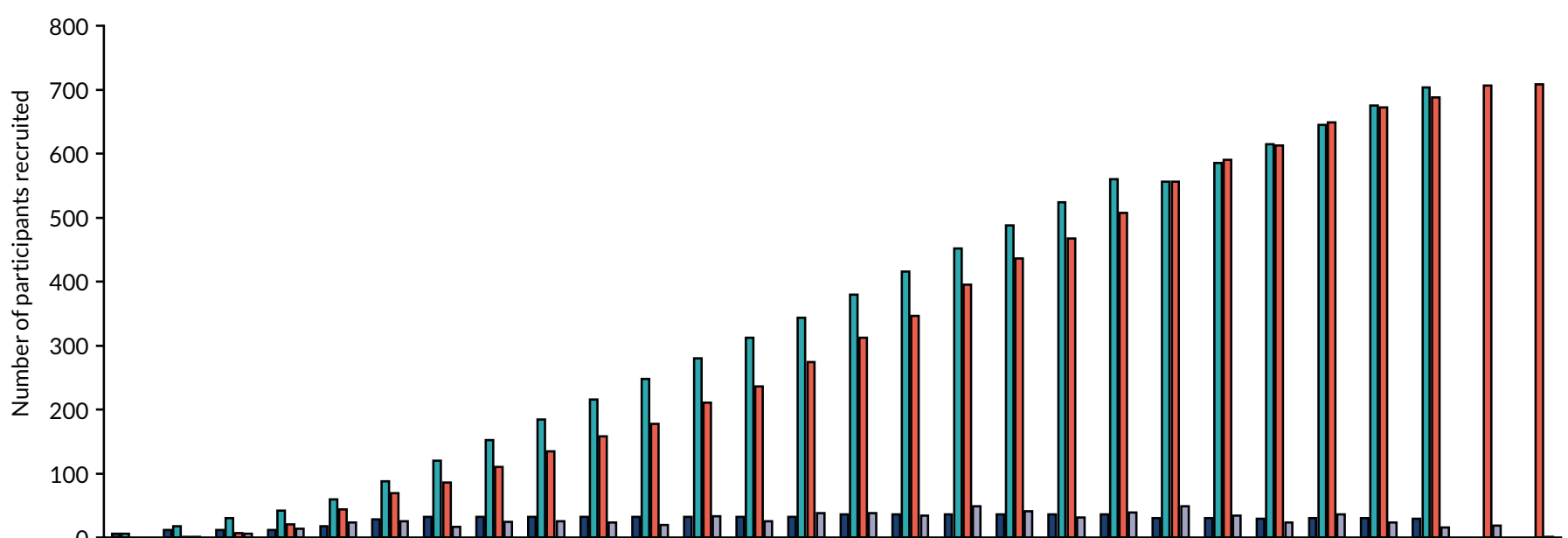

Predicted patients/month Predicted total

Actual patients/month

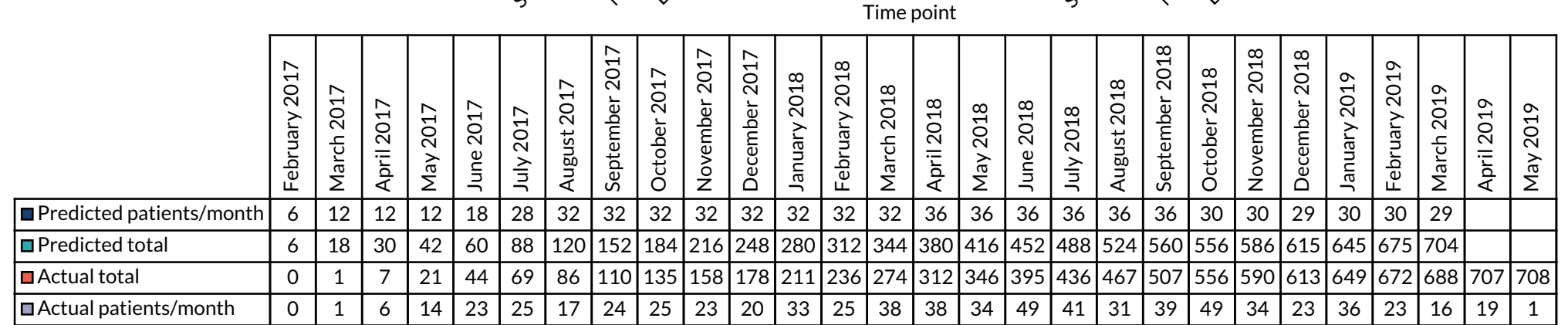


TABLE 31 Baseline characteristics detailed (inside the table)

\begin{tabular}{|c|c|c|c|c|c|c|c|c|}
\hline \multirow[b]{2}{*}{ Characteristic } & \multicolumn{2}{|c|}{$\begin{array}{l}\text { Best-practice } \\
\text { advice }(N=174)\end{array}$} & \multicolumn{2}{|c|}{$\begin{array}{l}\text { Injection plus } \\
\text { best-practice } \\
\text { advice ( } N=178)\end{array}$} & \multicolumn{2}{|c|}{$\begin{array}{l}\text { Progressive } \\
\text { exercise } \\
(N=174)\end{array}$} & \multicolumn{2}{|c|}{$\begin{array}{l}\text { Injection plus } \\
\text { progressive } \\
\text { exercise }(N=182)\end{array}$} \\
\hline & $n$ & $\%$ & $n$ & $\%$ & $n$ & $\%$ & $n$ & $\%$ \\
\hline \multicolumn{9}{|l|}{ Ethnicity } \\
\hline White British & 158 & 90.8 & 162 & 91.0 & 152 & 87.4 & 163 & 89.6 \\
\hline White other & 2 & 1.1 & 5 & 2.8 & 2 & 1.1 & 4 & 2.2 \\
\hline Mixed & 1 & 0.6 & 0 & 0.0 & 2 & 1.1 & 3 & 1.6 \\
\hline Indian & 4 & 2.3 & 3 & 1.7 & 3 & 1.7 & 4 & 2.2 \\
\hline Pakistani & 4 & 2.3 & 2 & 1.1 & 7 & 4.0 & 2 & 1.1 \\
\hline Bangladeshi & 1 & 0.6 & 0 & 0.0 & 0 & 0.0 & 1 & 0.5 \\
\hline Black or black British & 3 & 1.7 & 3 & 1.7 & 4 & 2.3 & 4 & 2.2 \\
\hline Chinese & 0 & 0.0 & 0 & 0.0 & 0 & 0.0 & 0 & 0.0 \\
\hline Other & 0 & 0.0 & 3 & 1.7 & 1 & 0.6 & 1 & 0.5 \\
\hline Prefer not to say & 1 & 0.6 & 0 & 0.0 & 1 & 0.6 & 0 & 0.0 \\
\hline Missing & 0 & 0.0 & 0 & 0.0 & 2 & 1.1 & 0 & 0.0 \\
\hline \multicolumn{9}{|l|}{ Marital status } \\
\hline Married/civil union & 118 & 67.8 & 107 & 60.1 & 114 & 65.5 & 120 & 65.9 \\
\hline Living with partner & 24 & 13.8 & 23 & 12.9 & 22 & 12.6 & 24 & 13.2 \\
\hline Unmarried (never married) & 10 & 5.7 & 11 & 6.2 & 14 & 8.0 & 17 & 9.3 \\
\hline Separated/divorced & 13 & 7.5 & 29 & 16.3 & 13 & 7.5 & 13 & 7.1 \\
\hline Widow/widower & 7 & 4.0 & 6 & 3.4 & 8 & 4.6 & 6 & 3.3 \\
\hline Prefer not to say & 2 & 1.1 & 2 & 1.1 & 1 & 0.6 & 2 & 1.1 \\
\hline Missing & 0 & 0.0 & 0 & 0.0 & 2 & 1.1 & 0 & 0.0 \\
\hline \multicolumn{9}{|l|}{ Current work status } \\
\hline Retired & 44 & 25.3 & 50 & 28.1 & 40 & 23.0 & 49 & 26.9 \\
\hline Semi-retired & 13 & 7.5 & 10 & 5.6 & 9 & 5.2 & 7 & 3.8 \\
\hline Employed & 84 & 48.3 & 91 & 51.1 & 98 & 56.3 & 82 & 45.1 \\
\hline Self-employed & 19 & 10.9 & 14 & 7.9 & 12 & 6.9 & 17 & 9.3 \\
\hline Unemployed & 2 & 1.1 & 5 & 2.8 & 5 & 2.9 & 9 & 4.9 \\
\hline Permanently sick or disabled & 4 & 2.3 & 1 & 0.6 & 1 & 0.6 & 4 & 2.2 \\
\hline Looking after home or family & 6 & 3.4 & 6 & 3.4 & 4 & 2.3 & 8 & 4.4 \\
\hline Other & 2 & 1.1 & 1 & 0.6 & 3 & 1.7 & 5 & 2.7 \\
\hline Missing & 0 & 0.0 & 0 & 0.0 & 2 & 1.1 & 1 & 0.5 \\
\hline \multicolumn{9}{|l|}{ Level of education } \\
\hline None or primary education & 2 & 1.1 & 4 & 2.2 & 4 & 2.3 & 6 & 3.3 \\
\hline Secondary & 92 & 52.9 & 92 & 51.7 & 74 & 42.5 & 89 & 48.9 \\
\hline $\begin{array}{l}\text { Higher professional or } \\
\text { university education }\end{array}$ & 80 & 46.0 & 82 & 46.1 & 94 & 54.0 & 87 & 47.8 \\
\hline Missing & 0 & 0.0 & 0 & 0.0 & 2 & 1.1 & 0 & 0.0 \\
\hline
\end{tabular}


TABLE 31 Baseline characteristics detailed (inside the table) (continued)

\begin{tabular}{|c|c|c|c|c|}
\hline & $\begin{array}{l}\text { Best-practice } \\
\text { advice }(N=174)\end{array}$ & $\begin{array}{l}\text { Injection plus } \\
\text { best-practice } \\
\text { advice }(N=178)\end{array}$ & $\begin{array}{l}\text { Progressive } \\
\text { exercise } \\
(N=174)\end{array}$ & $\begin{array}{l}\text { Injection plus } \\
\text { progressive } \\
\text { exercise }(N=182)\end{array}$ \\
\hline Characteristic & $\%$ & $\%$ & $\%$ & $\%$ \\
\hline
\end{tabular}

Household income

\begin{tabular}{|c|c|c|c|c|c|c|c|c|}
\hline$<£ 10,000$ & 36 & 20.7 & 27 & 15.2 & 22 & 12.6 & 27 & 14.8 \\
\hline $\begin{array}{l}\text { Between } £ 10,000 \text { and } \\
£ 19,999\end{array}$ & 39 & 22.4 & 60 & 33.7 & 46 & 26.4 & 52 & 28.6 \\
\hline $\begin{array}{l}\text { Between } £ 20,000 \text { and } \\
£ 29,000\end{array}$ & 41 & 23.6 & 26 & 14.6 & 36 & 20.7 & 30 & 16.5 \\
\hline $\begin{array}{l}\text { Between } £ 30,000 \text { and } \\
£ 39,999\end{array}$ & 21 & 12.1 & 23 & 12.9 & 20 & 11.5 & 23 & 12.6 \\
\hline $\begin{array}{l}\text { Between } £ 40,000 \text { and } \\
£ 49,999\end{array}$ & 12 & 6.9 & 12 & 6.7 & 8 & 4.6 & 14 & 7.7 \\
\hline$\geq £ 50,000$ & 12 & 6.9 & 10 & 5.6 & 19 & 10.9 & 10 & 5.5 \\
\hline Prefer not to answer & 13 & 7.5 & 20 & 11.2 & 20 & 11.5 & 25 & 13.7 \\
\hline Missing & 0 & 0.0 & 0 & 0.0 & 3 & 1.7 & 1 & 0.5 \\
\hline \multicolumn{9}{|l|}{ tate benefits } \\
\hline Yes & 37 & 21.3 & 36 & 20.2 & 30 & 17.2 & 41 & 22.5 \\
\hline No & 136 & 78.2 & 139 & 78.1 & 140 & 80.5 & 139 & 76.4 \\
\hline Prefer not to say & 1 & 0.6 & 3 & 1.7 & 2 & 1.1 & 1 & 0.5 \\
\hline Missing & 0 & 0.0 & 0 & 0.0 & 2 & 1.1 & 1 & 0.5 \\
\hline \multicolumn{9}{|l|}{ enefits received } \\
\hline Attendance Allowance & 1 & 0.6 & 2 & 1.1 & 0 & 0.0 & 0 & 0.0 \\
\hline Carer's Allowance & 6 & 3.4 & 2 & 1.1 & 2 & 1.1 & 2 & 1.1 \\
\hline Child Benefit & 16 & 9.2 & 14 & 7.9 & 14 & 8.0 & 14 & 7.7 \\
\hline Child Tax Credit & 8 & 4.6 & 9 & 5.1 & 8 & 4.6 & 3 & 1.6 \\
\hline Council Tax Benefit & 7 & 4.0 & 5 & 2.8 & 4 & 2.3 & 2 & 1.1 \\
\hline Disability Living Allowance & 5 & 2.9 & 6 & 3.4 & 3 & 1.7 & 7 & 3.8 \\
\hline $\begin{array}{l}\text { Employment and Support } \\
\text { Allowance }\end{array}$ & 2 & 1.1 & 4 & 2.2 & 2 & 1.1 & 8 & 4.4 \\
\hline Income Support & 3 & 1.7 & 1 & 0.6 & 2 & 1.1 & 3 & 1.6 \\
\hline Housing Benefit & 5 & 2.9 & 3 & 1.7 & 3 & 1.7 & 4 & 2.2 \\
\hline Jobseeker's Allowance & 2 & 1.1 & 1 & 0.6 & 0 & 0.0 & 1 & 0.5 \\
\hline Working Tax Credit & 4 & 2.3 & 7 & 3.9 & 5 & 2.9 & 5 & 2.7 \\
\hline Other & 10 & 5.7 & 9 & 5.1 & 8 & 4.6 & 9 & 4.9 \\
\hline
\end{tabular}


TABLE 32 Participant demographics at baseline: categorical outcomes (at the margins)

\begin{tabular}{|c|c|c|c|c|c|c|c|c|c|c|}
\hline \multirow[b]{3}{*}{ Participant demographic } & \multicolumn{4}{|c|}{ Progressive exercise } & \multicolumn{4}{|c|}{ Injection } & & \\
\hline & \multicolumn{2}{|c|}{$\begin{array}{l}\text { Best-practice } \\
\text { advice }\end{array}$} & \multicolumn{2}{|c|}{$\begin{array}{l}\text { Progressive } \\
\text { exercise }\end{array}$} & \multicolumn{2}{|l|}{ No } & \multicolumn{2}{|l|}{ Yes } & \multicolumn{2}{|c|}{ Overall } \\
\hline & $n$ & $\%$ & $n$ & $\%$ & $n$ & $\%$ & $n$ & $\%$ & $n$ & $\%$ \\
\hline \multicolumn{11}{|l|}{ Ethnicity } \\
\hline White British & 320 & 90.9 & 315 & 88.5 & 310 & 89.1 & 325 & 90.3 & 635 & 89.7 \\
\hline White other & 7 & 2.0 & 6 & 1.7 & 4 & 1.1 & 9 & 2.5 & 13 & 1.8 \\
\hline Mixed & 1 & 0.3 & 5 & 1.4 & 3 & 0.9 & 3 & 0.8 & 6 & 0.8 \\
\hline Indian & 7 & 2.0 & 7 & 2.0 & 7 & 2.0 & 7 & 1.9 & 14 & 2.0 \\
\hline Pakistani & 6 & 1.7 & 9 & 2.5 & 11 & 3.2 & 4 & 1.1 & 15 & 2.1 \\
\hline Bangladeshi & 1 & 0.3 & 1 & 0.3 & 1 & 0.3 & 1 & 0.3 & 2 & 0.3 \\
\hline Black or black British & 6 & 1.7 & 8 & 2.2 & 7 & 2.0 & 7 & 1.9 & 14 & 2.0 \\
\hline Chinese & 0 & 0.0 & 0 & 0.0 & 0 & 0.0 & 0 & 0.0 & 0 & 0.0 \\
\hline Other & 3 & 0.9 & 2 & 0.6 & 1 & 0.3 & 4 & 1.1 & 5 & 0.7 \\
\hline Missing & 0 & 0.0 & 2 & 0.6 & 2 & 0.6 & 0 & 0.0 & 2 & 0.3 \\
\hline \multicolumn{11}{|l|}{ Marital status } \\
\hline Married/civil union & 225 & 63.9 & 234 & 65.7 & 232 & 66.7 & 227 & 63.1 & 459 & 64.8 \\
\hline Living with partner & 47 & 13.4 & 46 & 12.9 & 46 & 13.2 & 47 & 13.1 & 93 & 13.1 \\
\hline $\begin{array}{l}\text { Unmarried (never } \\
\text { married) }\end{array}$ & 21 & 6.0 & 31 & 8.7 & 24 & 6.9 & 28 & 7.8 & 52 & 7.3 \\
\hline Separated/divorced & 42 & 11.9 & 26 & 7.3 & 26 & 7.5 & 42 & 11.7 & 68 & 9.6 \\
\hline Widow/widower & 13 & 3.7 & 14 & 3.9 & 15 & 4.3 & 12 & 3.3 & 27 & 3.8 \\
\hline Prefer not to say & 4 & 1.1 & 3 & 0.8 & 3 & 0.9 & 4 & 1.1 & 7 & 1.0 \\
\hline Missing & 0 & 0.0 & 2 & 0.6 & 2 & 0.6 & 0 & 0.0 & 2 & 0.3 \\
\hline \multicolumn{11}{|l|}{ Body mass index $\left(\mathrm{kg} / \mathrm{m}^{2}\right)$} \\
\hline Underweight $(<18.5)$ & 4 & 1.1 & 0 & 0.0 & 3 & 0.9 & 1 & 0.3 & 4 & 0.6 \\
\hline $\begin{array}{l}\text { Normal weight } \\
(18.5-24.9)\end{array}$ & 102 & 29.0 & 103 & 28.9 & 101 & 29.0 & 104 & 28.9 & 205 & 29.0 \\
\hline Overweight (25-29.9) & 131 & 37.2 & 143 & 40.2 & 141 & 40.5 & 133 & 36.9 & 274 & 38.7 \\
\hline Obese $(\geq 30)$ & 113 & 32.1 & 104 & 29.2 & 99 & 28.4 & 118 & 32.8 & 217 & 30.6 \\
\hline Missing & 2 & 0.6 & 6 & 1.7 & 4 & 1.1 & 4 & 1.1 & 8 & 1.1 \\
\hline \multicolumn{11}{|l|}{ Smoking status } \\
\hline Never smoked & 185 & 52.6 & 200 & 56.2 & 184 & 52.9 & 201 & 55.8 & 635 & 89.7 \\
\hline Former smoker & 132 & 37.5 & 124 & 34.8 & 127 & 36.5 & 129 & 35.8 & 13 & 1.8 \\
\hline Current smoker & 35 & 9.9 & 30 & 8.4 & 35 & 10.1 & 30 & 8.3 & 6 & 0.8 \\
\hline Missing & 0 & 0.0 & 2 & 0.6 & 2 & 0.6 & 0 & 0.0 & 2 & 0.3 \\
\hline
\end{tabular}


TABLE 32 Participant demographics at baseline: categorical outcomes (at the margins) (continued)

\begin{tabular}{|c|c|c|c|c|c|c|c|}
\hline \multirow[b]{3}{*}{ Participant demographic } & \multicolumn{2}{|c|}{ Progressive exercise } & \multicolumn{4}{|c|}{ Injection } & \multirow[b]{2}{*}{ Overall } \\
\hline & $\begin{array}{l}\text { Best-practice } \\
\text { advice }\end{array}$ & $\begin{array}{l}\text { Progressive } \\
\text { exercise }\end{array}$ & No & & Ye: & & \\
\hline & $\%$ & $\%$ & $n$ & $\%$ & $n$ & $\%$ & $\%$ \\
\hline
\end{tabular}

Affected shoulder

Left shoulder
Right shoulder
Both shoulders
Missing

Hand dominance

Left handed

Right handed

Both

Missing

Current work status

Retired

Semi-retired

Employed

Self-employed

Unemployed

Permanently sick or disabled

Looking after home or family

Other

Missing

Level of education

None or primary education

Secondary

Higher professional or university education

Missing

Household income

$$
\leq £ 10,000
$$

Between $£ 10,000$ and $£ 19,999$

Between $£ 20,000$ and $£ 29,000$

$\begin{array}{rrrrrrrrrr}167 & 47.4 & 165 & 46.3 & 172 & 49.4 & 160 & 44.4 & 332 & 46.9 \\ 172 & 48.9 & 177 & 49.7 & 162 & 46.6 & 187 & 51.9 & 349 & 49.3 \\ 13 & 3.7 & 12 & 3.4 & 12 & 3.4 & 13 & 3.6 & 25 & 3.5 \\ 0 & 0.0 & 2 & 0.6 & 2 & 0.6 & 0 & 0.0 & 2 & 0.3\end{array}$

$\begin{array}{rrrrrrrrrr}29 & 8.2 & 42 & 11.8 & 34 & 9.8 & 37 & 10.3 & 71 & 10.0 \\ 310 & 88.1 & 306 & 86.0 & 305 & 87.6 & 311 & 86.4 & 616 & 87.0 \\ 13 & 3.7 & 6 & 1.7 & 7 & 2.0 & 12 & 3.3 & 19 & 2.7 \\ 0 & 0.0 & 2 & 0.6 & 2 & 0.6 & 0 & 0.0 & 2 & 0.3\end{array}$

$\begin{array}{rrrrrrrrrr}94 & 26.7 & 89 & 25.0 & 84 & 24.1 & 99 & 27.5 & 183 & 25.8 \\ 23 & 6.5 & 16 & 4.5 & 22 & 6.3 & 17 & 4.7 & 39 & 5.5 \\ 175 & 49.7 & 180 & 50.6 & 182 & 52.3 & 173 & 48.1 & 355 & 50.1 \\ 33 & 9.4 & 29 & 8.1 & 31 & 8.9 & 31 & 8.6 & 62 & 8.8 \\ 7 & 2.0 & 14 & 3.9 & 7 & 2.0 & 14 & 3.9 & 21 & 3.0 \\ 5 & 1.4 & 5 & 1.4 & 5 & 1.4 & 5 & 1.4 & 10 & 1.4 \\ 12 & 3.4 & 12 & 3.4 & 10 & 2.9 & 14 & 3.9 & 24 & 3.4 \\ 3 & 0.9 & 8 & 2.2 & 5 & 1.4 & 6 & 1.7 & 11 & 1.6 \\ 0 & 0.0 & 3 & 0.8 & 2 & 0.6 & 1 & 0.3 & 3 & 0.4\end{array}$

$\begin{array}{rrrrrrrrrrr}6 & 1.7 & 10 & 2.8 & 6 & 1.7 & 10 & 2.8 & 16 & 2.3 \\ 184 & 52.3 & 163 & 45.8 & 166 & 47.7 & 181 & 50.3 & 347 & 49.0 \\ 162 & 46.0 & 181 & 50.8 & 174 & 50.0 & 169 & 46.9 & 343 & 48.4 \\ 0 & 0.0 & 2 & 0.6 & 2 & 0.6 & 0 & 0.0 & 2 & 0.3 \\ 63 & 17.9 & 49 & 13.8 & 58 & 16.7 & 54 & 15.0 & 112 & 15.8 \\ 99 & 28.1 & 98 & 27.5 & 85 & 24.4 & 112 & 31.1 & 197 & 27.8 \\ 67 & 19.0 & 66 & 18.5 & 77 & 22.1 & 56 & 15.6 & 133 & 18.8\end{array}$


TABLE 32 Participant demographics at baseline: categorical outcomes (at the margins) (continued)

\begin{tabular}{|c|c|c|c|c|c|c|c|c|c|c|}
\hline \multirow[b]{3}{*}{ Participant demographic } & \multicolumn{4}{|c|}{ Progressive exercise } & \multicolumn{4}{|c|}{ Injection } & \multirow{2}{*}{\multicolumn{2}{|c|}{ Overall }} \\
\hline & \multicolumn{2}{|c|}{$\begin{array}{l}\text { Best-practice } \\
\text { advice }\end{array}$} & \multicolumn{2}{|c|}{$\begin{array}{l}\text { Progressive } \\
\text { exercise }\end{array}$} & \multicolumn{2}{|l|}{ No } & \multicolumn{2}{|l|}{ Yes } & & \\
\hline & $n$ & $\%$ & $n$ & $\%$ & $n$ & $\%$ & $n$ & $\%$ & $n$ & $\%$ \\
\hline $\begin{array}{l}\text { Between } £ 30,000 \text { and } \\
£ 39,999\end{array}$ & 44 & 12.5 & 43 & 12.1 & 41 & 11.8 & 46 & 12.8 & 87 & 12.3 \\
\hline $\begin{array}{l}\text { Between } £ 40,000 \text { and } \\
£ 49,999\end{array}$ & 24 & 6.8 & 22 & 6.2 & 20 & 5.7 & 26 & 7.2 & 46 & 6.5 \\
\hline$\geq £ 50,000$ & 22 & 6.3 & 29 & 8.1 & 31 & 8.9 & 20 & 5.6 & 51 & 7.2 \\
\hline Prefer not to answer & 33 & 9.4 & 45 & 12.6 & 33 & 9.5 & 45 & 12.5 & 78 & 11.0 \\
\hline Missing & 0 & 0.0 & 4 & 1.1 & 3 & 0.9 & 1 & 0.3 & 4 & 0.6 \\
\hline \multicolumn{11}{|l|}{ State benefits } \\
\hline Yes & 73 & 20.7 & 71 & 19.9 & 67 & 19.3 & 77 & 21.4 & 114 & 20.3 \\
\hline No & 275 & 78.1 & 279 & 78.4 & 276 & 79.3 & 278 & 77.2 & 554 & 78.2 \\
\hline Prefer not to say & 4 & 1.1 & 3 & 0.8 & 3 & 0.9 & 4 & 1.1 & 7 & 10 \\
\hline Missing & 0 & 0.0 & 3 & 0.8 & 2 & 0.6 & 1 & 0.3 & 3 & 0.4 \\
\hline \multicolumn{11}{|l|}{ Benefits received } \\
\hline Attendance Allowance & 3 & 0.9 & 0 & 0.0 & 1 & 0.3 & 2 & 0.6 & 3 & 0.4 \\
\hline Carer's Allowance & 8 & 2.3 & 4 & 1.1 & 8 & 2.3 & 4 & 1.1 & 12 & 1.7 \\
\hline Child Benefit & 30 & 8.5 & 28 & 7.9 & 30 & 8.6 & 28 & 7.8 & 58 & 8.2 \\
\hline Child Tax Credit & 17 & 4.8 & 11 & 3.1 & 16 & 4.6 & 12 & 3.3 & 28 & 4.0 \\
\hline Council Tax Benefit & 12 & 3.4 & 6 & 1.7 & 11 & 3.2 & 7 & 1.9 & 18 & 2.5 \\
\hline $\begin{array}{l}\text { Disability Living } \\
\text { Allowance }\end{array}$ & 11 & 3.1 & 10 & 2.8 & 8 & 2.3 & 13 & 3.6 & 21 & 3.0 \\
\hline $\begin{array}{l}\text { Employment and Support } \\
\text { Allowance }\end{array}$ & 6 & 1.7 & 10 & 2.8 & 4 & 1.1 & 12 & 3.3 & 16 & 2.3 \\
\hline Income Support & 4 & 1.1 & 5 & 1.4 & 5 & 1.4 & 4 & 1.1 & 9 & 1.3 \\
\hline Housing Benefit & 8 & 2.3 & 7 & 2.0 & 8 & 2.3 & 7 & 1.9 & 15 & 2.1 \\
\hline Jobseeker's Allowance & 3 & 0.9 & 1 & 0.3 & 2 & 0.6 & 2 & 0.6 & 4 & 0.6 \\
\hline Working Tax Credit & 11 & 3.1 & 10 & 2.8 & 9 & 2.6 & 12 & 3.3 & 21 & 3.0 \\
\hline Other & 19 & 5.4 & 17 & 4.8 & 18 & 5.2 & 18 & 5.0 & 36 & 5.1 \\
\hline
\end{tabular}


TABLE 33 Participant demographic characteristics at baseline: continuous outcomes (at the margins)

\begin{tabular}{|c|c|c|c|c|c|}
\hline \multirow[b]{2}{*}{ Characteristic } & \multicolumn{2}{|c|}{ Progressive exercise } & \multicolumn{2}{|l|}{ Injection } & \multirow[b]{2}{*}{$\begin{array}{l}\text { Overall } \\
(N=708)\end{array}$} \\
\hline & $\begin{array}{l}\text { Best-practice } \\
\text { advice }(N=352)\end{array}$ & $\begin{array}{l}\text { Progressive } \\
\text { exercise } \\
(N=356)\end{array}$ & No $(N=348)$ & Yes $(N=360)$ & \\
\hline Height $(\mathrm{m})$, mean (SD), $n$ & $1.7(0.0), 352$ & $1.7(0.0), 354$ & $1.7(0.0), 346$ & $1.7(0.2), 360$ & 1.7 (0.0), 706 \\
\hline Weight (kg), mean (SD), $n$ & 81.9 (17.0), 350 & 81.5 (18.2), 350 & 81.1 (17.6), 344 & 82.3 (17.6), 356 & 81.7 (17.6), 700 \\
\hline $\begin{array}{l}\text { Body mass index }\left(\mathrm{kg} / \mathrm{m}^{2}\right) \text {, } \\
\text { mean (SD), } n\end{array}$ & 28.3 (5.2), 350 & 28.0 (5.2), 350 & 27.9 (5.2), 344 & 28.4 (5.2), 356 & 28.2 (5.2), 700 \\
\hline Age (years), mean (SD), $n$ & 56.2 (12.7), 352 & 54.7 (13.5), 356 & 55.3 (13.4), 348 & 55.6 (12.8), 360 & 55.5 (13.1), 708 \\
\hline $\begin{array}{l}\text { Cigarettes smoked per } \\
\text { day (current smoker), } \\
\text { median (IQR), } n\end{array}$ & 10 (9-15), 32 & 10 (6-15), 29 & 10 (10-15), 33 & 10 (6-15), 28 & 10 (8-15), 61 \\
\hline $\begin{array}{l}\text { Cigarettes smoked per } \\
\text { day (former smoker), } \\
\text { median (IQR), } n\end{array}$ & 15.0 (7-20), 132 & 10.0 (5-20), 123 & $12.0(5-20), 126$ & 10.0 (8-20), 129 & 12.0 (6-20), 255 \\
\hline $\begin{array}{l}\text { Symptoms duration, } \\
\text { median (IQR), } n\end{array}$ & $4.0(2-6), 351$ & $4.0(3-6), 354$ & $4.0(3-6), 345$ & $4.0(3-6), 360$ & $4.0(3-6), 705$ \\
\hline \multicolumn{6}{|l|}{ SPADI, mean (SD), $n$} \\
\hline Pain subscale & 65.1 (18.0), 352 & 62.7 (17.4), 354 & 63.3 (17.6), 346 & 64.4 (17.9), 360 & 63.9 (17.7), 706 \\
\hline Function subscale & 47.2 (22.6), 352 & 41.5 (21.3), 354 & 44.2 (22.4), 346 & 44.5 (21.9), 360 & 44.3 (22.1), 706 \\
\hline SPADI overall & 56.1 (19.0), 352 & 52.1 (17.7), 354 & 53.8 (18.6), 346 & 54.4 (18.4), 360 & 54.1 (18.5), 706 \\
\hline FABQ-PA, mean (SD), $n$ & $15.6(5.6), 349$ & $14.5(5.4), 354$ & 14.9 (5.7), 344 & $15.2(5.3), 359$ & $15.1(5.5), 703$ \\
\hline PSEQ-2, mean (SD), $n$ & 9.6 (2.4), 352 & 9.8 (2.3), 354 & 9.7 (2.3), 346 & 9.6 (2.3), 360 & 9.7 (2.3), 706 \\
\hline ISI, mean (SD), $n$ & 10.7 (6.4), 349 & 10.3 (6.1), 350 & 10.2 (6.3), 343 & 10.7 (6.3), 356 & 10.5 (6.3), 699 \\
\hline \multicolumn{6}{|l|}{ RDA, mean (SD), $n$} \\
\hline Overall & 8.1 (2.6), 352 & 7.7 (2.6), 354 & 7.8 (2.7), 346 & 7.9 (2.5), 360 & 7.9 (2.6), 706 \\
\hline Recreational & 3.0 (1.0), 352 & 2.8 (1.0), 354 & 2.9 (1.0), 346 & 2.9 (1.0), 360 & 2.9 (1.0), 706 \\
\hline Social life & 2.5 (1.1), 352 & 2.3 (1.1), 354 & 2.3 (1.1), 346 & 2.5 (1.1), 360 & 2.4 (1.1), 706 \\
\hline Work & 2.6 (1.0), 352 & 2.5 (1.0), 354 & 2.6 (1.0), 346 & 2.6 (1.0), 360 & 2.6 (1.0), 706 \\
\hline
\end{tabular}


TABLE 34 Stratification factors (at the margins)

\begin{tabular}{|c|c|c|c|c|c|c|c|c|c|c|}
\hline \multirow[b]{3}{*}{ Factor } & \multicolumn{4}{|c|}{ Progressive exercise } & \multicolumn{4}{|c|}{ Injection } & & \\
\hline & \multicolumn{2}{|c|}{ Best-practice advice } & \multicolumn{2}{|c|}{ Progressive exercise } & \multicolumn{2}{|l|}{ No } & \multicolumn{2}{|l|}{ Yes } & \multicolumn{2}{|c|}{ Overall } \\
\hline & $n$ & $\%$ & $n$ & $\%$ & $n$ & $\%$ & $n$ & $\%$ & $n$ & $\%$ \\
\hline \multicolumn{11}{|l|}{ Sex } \\
\hline Male & 176 & 50.0 & 183 & 51.4 & 177 & 50.9 & 182 & 50.6 & 359 & 50.7 \\
\hline Female & 176 & 50.0 & 173 & 48.6 & 171 & 49.1 & 178 & 49.4 & 349 & 49.3 \\
\hline \multicolumn{11}{|c|}{ Age group (years) } \\
\hline $18-35$ & 24 & 6.8 & 31 & 8.7 & 25 & 7.2 & 30 & 8.3 & 55 & 7.8 \\
\hline$\geq 36$ & 328 & 93.2 & 325 & 91.3 & 323 & 92.8 & 330 & 91.7 & 653 & 92.2 \\
\hline \multicolumn{11}{|l|}{ Trial centre } \\
\hline 1 & 21 & 6.0 & 25 & 7.0 & 24 & 6.9 & 22 & 6.1 & 46 & 6.5 \\
\hline 2 & 52 & 14.8 & 50 & 14.0 & 51 & 14.7 & 51 & 14.2 & 102 & 14.4 \\
\hline 3 & 59 & 16.8 & 61 & 17.1 & 60 & 17.2 & 60 & 16.7 & 120 & 16.9 \\
\hline 4 & 15 & 4.3 & 14 & 3.9 & 15 & 4.3 & 14 & 3.9 & 29 & 4.1 \\
\hline 5 & 17 & 4.8 & 18 & 5.1 & 17 & 4.9 & 18 & 5.0 & 35 & 4.9 \\
\hline 6 & 6 & 1.7 & 8 & 2.2 & 7 & 2.0 & 7 & 1.9 & 14 & 2.0 \\
\hline 7 & 7 & 2.0 & 7 & 2.0 & 6 & 1.7 & 8 & 2.2 & 14 & 2.0 \\
\hline 8 & 11 & 3.1 & 12 & 3.4 & 12 & 3.4 & 11 & 3.1 & 23 & 3.2 \\
\hline 9 & 7 & 2.0 & 6 & 1.7 & 8 & 2.3 & 5 & 1.4 & 13 & 1.8 \\
\hline 10 & 14 & 4.0 & 17 & 4.8 & 15 & 4.3 & 16 & 4.4 & 31 & 4.4 \\
\hline 11 & 2 & 0.6 & 2 & 0.6 & 2 & 0.6 & 2 & 0.6 & 4 & 0.6 \\
\hline 12 & 24 & 6.8 & 25 & 7.0 & 25 & 7.2 & 24 & 6.7 & 49 & 6.9 \\
\hline 13 & 16 & 4.5 & 17 & 4.8 & 15 & 4.3 & 18 & 5.0 & 33 & 4.7 \\
\hline 14 & 16 & 4.5 & 13 & 3.7 & 13 & 3.7 & 16 & 4.4 & 29 & 4.1 \\
\hline 15 & 18 & 5.1 & 18 & 5.1 & 17 & 4.9 & 19 & 5.3 & 36 & 5.1 \\
\hline 16 & 19 & 5.4 & 17 & 4.8 & 17 & 4.9 & 19 & 5.3 & 36 & 5.1 \\
\hline 17 & 13 & 3.7 & 12 & 3.4 & 12 & 3.4 & 13 & 3.6 & 25 & 3.5 \\
\hline 18 & 20 & 5.7 & 20 & 5.6 & 18 & 5.2 & 22 & 6.1 & 40 & 5.6 \\
\hline 19 & 5 & 1.4 & 6 & 1.7 & 5 & 1.4 & 6 & 1.7 & 11 & 1.6 \\
\hline 20 & 10 & 2.8 & 8 & 2.2 & 9 & 2.6 & 9 & 2.5 & 18 & 2.5 \\
\hline
\end{tabular}

TABLE 35 Response rates

\begin{tabular}{llll} 
Response rate & $\mathbf{8}$ weeks $(\mathbf{N}=\mathbf{7 0 8}), \boldsymbol{n}(\%)$ & $\mathbf{6}$ months $(\mathbf{N}=\mathbf{7 0 8}), \boldsymbol{n}(\%)$ & $\mathbf{1 2}$ months (N= 708), $\boldsymbol{n}(\%)$ \\
Questionnaires posted & $706(99)$ & $698(99)$ & $694(98)$ \\
Overall response & $642(91)$ & $615(87)$ & $618(87)$ \\
$\quad$ Postal response & $563(80)$ & $524(74)$ & $516(73)$ \\
$\quad$ Telephone response & $79(11)$ & $82(12)$ & $88(12)$ \\
$\quad$ Electronic response & - & $9(1)$ & $14(2)$ \\
Non-responder (missing data) & $67(9)$ & $93(13)$ & $90(13)$ \\
\hline
\end{tabular}


TABLE 36 Timing of intervention delivery

\begin{tabular}{|c|c|c|c|c|}
\hline Period & $\begin{array}{l}\text { Injection plus } \\
\text { best-practice } \\
\text { advice, median } \\
\text { (IQR) }\end{array}$ & $\begin{array}{l}\text { Best-practice } \\
\text { advice only, } \\
\text { median (IQR) }\end{array}$ & $\begin{array}{l}\text { Injection plus } \\
\text { progressive } \\
\text { exercise, } \\
\text { median (IQR) }\end{array}$ & $\begin{array}{l}\text { Progressive } \\
\text { exercise only, } \\
\text { median (IQR) }\end{array}$ \\
\hline Randomisation to injection attendance (days) & $6(0-9)$ & & $7(0-10)$ & \\
\hline $\begin{array}{l}\text { Randomisation to first exercise session } \\
\text { attended (days) }\end{array}$ & $21(15-28)$ & $15(12-21)$ & $22(17-28)$ & $16(10-21)$ \\
\hline Randomisation to last exercise session (days) & & & $103(68-135)$ & $92(64-121)$ \\
\hline First to last exercise session attended (days) & & & $78(41-105)$ & $77(49-106)$ \\
\hline
\end{tabular}

TABLE 37 Exercises prescribed: progressive-exercise intervention

\begin{tabular}{|c|c|c|c|c|c|c|c|c|}
\hline \multirow[b]{2}{*}{ Level } & \multirow[b]{2}{*}{ Exercise } & \multicolumn{7}{|c|}{ Exercises prescribed, $n^{a}$} \\
\hline & & Session 1 & Session 2 & Session 3 & Session 4 & Session 5 & Session 6 & $\begin{array}{l}\text { Total, } \\
n(\%)\end{array}$ \\
\hline \multirow[t]{6}{*}{1} & $\begin{array}{l}\text { Shoulder flexion } \\
\text { supported by table }\end{array}$ & 13 & 5 & 2 & 3 & 3 & 0 & $26(1)$ \\
\hline & $\begin{array}{l}\text { Shoulder abduction } \\
\text { supported by table }\end{array}$ & 34 & 11 & 6 & 6 & 4 & 1 & $62(2)$ \\
\hline & $\begin{array}{l}\text { Shoulder flexion } \\
\text { supported by table in } \\
\text { standing }\end{array}$ & 18 & 4 & 3 & 2 & 1 & 0 & $28(1)$ \\
\hline & $\begin{array}{l}\text { Shoulder abduction } \\
\text { supported by table in } \\
\text { standing }\end{array}$ & 15 & 6 & 5 & 2 & 1 & 1 & $30(1)$ \\
\hline & $\begin{array}{l}\text { Shoulder flexion } \\
\text { (self-assisted): supine }\end{array}$ & 18 & 14 & 5 & 3 & 1 & 0 & $41(1)$ \\
\hline & $\begin{array}{l}\text { Shoulder flexion } \\
\text { (self-assisted): up a wall }\end{array}$ & 43 & 21 & 12 & 7 & 4 & 2 & 89 (3) \\
\hline \multirow[t]{8}{*}{2 : core } & $\begin{array}{l}\text { Isometric shoulder } \\
\text { external rotation }\end{array}$ & 70 & 38 & 28 & 13 & 3 & 4 & $156(5)$ \\
\hline & $\begin{array}{l}\text { Isometric shoulder } \\
\text { abduction }\end{array}$ & 41 & 25 & 18 & 9 & 6 & 3 & $102(3)$ \\
\hline & $\begin{array}{l}{ }^{\mathrm{b}} \text { Resisted shoulder } \\
\text { external rotation; } 0^{\circ}\end{array}$ & 138 & 112 & 71 & 33 & 24 & 16 & 394 (12) \\
\hline & $\begin{array}{l}\text { besisted shoulder } \\
\text { abduction }\end{array}$ & 74 & 92 & 72 & 51 & 36 & 17 & 342 (10) \\
\hline & Shoulder flexion up a wall & 38 & 33 & 26 & 12 & 7 & 7 & $123(4)$ \\
\hline & $\begin{array}{l}\text { Shoulder raise using a } \\
\text { weight }\end{array}$ & 27 & 44 & 37 & 27 & 22 & 13 & $170(5)$ \\
\hline & $\begin{array}{l}\text { besisted shoulder } \\
\text { external rotation } \\
\text { (supported): } 90^{\circ}\end{array}$ & 25 & 36 & 42 & 32 & 19 & 10 & $164(5)$ \\
\hline & $\begin{array}{l}{ }^{\mathrm{b}} \text { Resisted shoulder } \\
\text { external rotation } \\
\text { (unsupported): } 90^{\circ}\end{array}$ & 19 & 34 & 28 & 28 & 25 & 18 & $152(5)$ \\
\hline
\end{tabular}


TABLE 37 Exercises prescribed: progressive-exercise intervention (continued)

\begin{tabular}{|c|c|c|c|c|c|c|c|c|}
\hline \multirow[b]{2}{*}{ Level } & \multirow[b]{2}{*}{ Exercise } & \multicolumn{7}{|c|}{ Exercises prescribed, $n^{a}$} \\
\hline & & Session 1 & Session 2 & Session 3 & Session 4 & Session 5 & Session 6 & $\begin{array}{l}\text { Total, } \\
\text { n (\%) }\end{array}$ \\
\hline \multirow{13}{*}{ 2: optional } & $\begin{array}{l}\text { bBilateral shoulder } \\
\text { flexion/abduction }\end{array}$ & 119 & 136 & 116 & 77 & 46 & 26 & $520(16)$ \\
\hline & $\begin{array}{l}{ }^{b} \text { Resisted shoulder } \\
\text { abduction/external } \\
\text { rotation }\end{array}$ & 32 & 37 & 43 & 28 & 15 & 12 & $167(5)$ \\
\hline & $\begin{array}{l}\text { Isometric shoulder } \\
\text { internal rotation }\end{array}$ & 9 & 12 & 8 & 6 & 3 & 0 & $38(1)$ \\
\hline & $\begin{array}{l}{ }^{\mathrm{b}} \text { Resisted shoulder } \\
\text { internal rotation: } 0^{\circ}\end{array}$ & 13 & 12 & 11 & 8 & 6 & 5 & $55(2)$ \\
\hline & $\begin{array}{l}{ }^{b} \text { Resisted shoulder } \\
\text { internal rotation } \\
\text { (supported): } 90^{\circ}\end{array}$ & 8 & 13 & 16 & 15 & 6 & 2 & $60(2)$ \\
\hline & $\begin{array}{l}{ }^{\mathrm{b}} \text { Resisted shoulder } \\
\text { internal rotation } \\
\text { (unsupported): } 90^{\circ}\end{array}$ & 7 & 7 & 13 & 9 & 14 & 10 & $60(2)$ \\
\hline & $\begin{array}{l}{ }^{b} \text { Resisted shoulder } \\
\text { adduction/internal } \\
\text { rotation }\end{array}$ & 0 & 3 & 11 & 6 & 7 & 4 & $31(1)$ \\
\hline & $\begin{array}{l}\text { Shoulder retraction: } \\
\text { prone }\end{array}$ & 9 & 7 & 9 & 11 & 8 & 5 & $49(1)$ \\
\hline & $\begin{array}{l}\text { Shoulder flexion (elbow } \\
\text { bent): prone }\end{array}$ & 0 & 7 & 2 & 5 & 1 & 3 & $18(1)$ \\
\hline & $\begin{array}{l}\text { Shoulder flexion } \\
\text { (elbow straight): prone }\end{array}$ & 1 & 3 & 6 & 4 & 1 & 0 & $15(0)$ \\
\hline & $\begin{array}{l}\text { Shoulder flexion } \\
\text { (crawl position) }\end{array}$ & 9 & 14 & 16 & 13 & 11 & 7 & $70(2)$ \\
\hline & Press-ups (on knees) & 14 & 17 & 17 & 17 & 15 & 7 & $87(3)$ \\
\hline & Press-ups (normal) & 3 & 7 & 7 & 6 & 7 & 1 & $31(1)$ \\
\hline \multirow{2}{*}{$\begin{array}{l}\text { Optional } \\
\text { stretches }\end{array}$} & Sleeper stretch & 9 & 11 & 13 & 10 & 5 & 2 & $50(1)$ \\
\hline & $\begin{array}{l}\text { Horizontal adduction } \\
\text { stretch }\end{array}$ & 5 & 4 & 6 & 4 & 3 & 1 & $23(1)$ \\
\hline 3 & $\begin{array}{l}\text { Therapist-designed } \\
\text { exercise(s) }\end{array}$ & 7 & 25 & 43 & 40 & 33 & 20 & $168(5)$ \\
\hline \multicolumn{2}{|c|}{ Unknown exercise } & 1 & 7 & 0 & 2 & 5 & 3 & $18(1)$ \\
\hline \multicolumn{2}{|l|}{ Total, n (\%) } & $819(25)$ & $797(24)$ & $692(21)$ & $489(15)$ & $342(10)$ & $200(6)$ & 3339 (100) \\
\hline
\end{tabular}


TABLE 38 Progression of exercise intervention: progressive-exercise intervention

\begin{tabular}{|c|c|c|c|}
\hline Progression of exercise & $\begin{array}{l}\text { Progressive exercise } \\
\text { only, } \boldsymbol{n}(\%)\end{array}$ & $\begin{array}{l}\text { Injection plus progressive } \\
\text { exercise, } \boldsymbol{n}(\%)\end{array}$ & Total, $\boldsymbol{n}(\%)$ \\
\hline Progressed $^{a}$ & $118(71)$ & $109(63)$ & $227(67)$ \\
\hline Maintained $^{\mathrm{b}}$ & $34(20)$ & $39(23)$ & $73(22)$ \\
\hline Regressed $^{c}$ & $15(9)$ & $24(14)$ & $39(12)$ \\
\hline Total & $167(100)$ & $172(100)$ & 339 (100) \\
\hline \multicolumn{4}{|c|}{$\begin{array}{l}\text { a Progression defined as an increase in exercise difficulty level, or either volume and/or load across attended } \\
\text { exercise sessions. } \\
\text { b Maintained defined as no change in any of exercise difficulty level, or either volume and/or load across attended } \\
\text { exercise sessions. If volume increased and load decreased (or vice versa), the exercise was considered to have been } \\
\text { maintained (assuming exercise difficulty level remained the same). } \\
\text { c Regression defined as a decrease in exercise difficulty level, or either volume and/or load across attended } \\
\text { exercise sessions. }\end{array}$} \\
\hline
\end{tabular}

TABLE 39 Participant-reported exercise adherence

\begin{tabular}{|c|c|c|c|c|c|c|c|c|c|c|}
\hline \multirow[b]{2}{*}{ Adherence } & \multicolumn{2}{|c|}{ Best-practice advice } & \multicolumn{2}{|c|}{ Progressive exercise } & \multicolumn{2}{|c|}{ No injection } & \multicolumn{2}{|c|}{ Injection } & \multicolumn{2}{|c|}{ Overall } \\
\hline & $n$ & $\%$ & $n$ & $\%$ & $n$ & $\%$ & $n$ & $\%$ & $n$ & $\%$ \\
\hline \multicolumn{11}{|l|}{8 weeks } \\
\hline Every day & 34 & 9.7 & 40 & 11.2 & 34 & 9.8 & 40 & 11.1 & 74 & 10.5 \\
\hline 6 days per week & 15 & 4.3 & 15 & 4.2 & 15 & 4.3 & 15 & 4.2 & 30 & 4.2 \\
\hline 5 days per week & 152 & 43.2 & 215 & 60.4 & 160 & 46.0 & 207 & 57.5 & 367 & 51.8 \\
\hline 4 days per week & 26 & 7.4 & 19 & 5.3 & 27 & 7.8 & 18 & 5.0 & 45 & 6.4 \\
\hline 3 days per week & 15 & 4.3 & 6 & 1.7 & 15 & 4.3 & 6 & 1.7 & 21 & 3.0 \\
\hline 2 days per week & 9 & 2.6 & 2 & 0.6 & 6 & 1.7 & 5 & 1.4 & 11 & 1.6 \\
\hline 1 day per week & 3 & 0.9 & 0 & 0.0 & 1 & 0.3 & 2 & 0.6 & 3 & 0.4 \\
\hline None & 28 & 8.0 & 11 & 3.1 & 18 & 5.2 & 21 & 5.8 & 39 & 5.5 \\
\hline Missing & 70 & 19.9 & 48 & 13.5 & 72 & 20.7 & 46 & 12.8 & 118 & 16.7 \\
\hline \multicolumn{11}{|l|}{6 months } \\
\hline Every day & 19 & 5.4 & 14 & 3.9 & 14 & 4.0 & 19 & 5.3 & 33 & 4.7 \\
\hline 6 days per week & 5 & 1.4 & 4 & 1.1 & 7 & 2.0 & 2 & 0.6 & 9 & 1.3 \\
\hline 5 days per week & 53 & 15.1 & 66 & 18.5 & 42 & 12.1 & 77 & 21.4 & 119 & 16.8 \\
\hline 4 days per week & 24 & 6.8 & 26 & 7.3 & 22 & 6.3 & 28 & 7.8 & 50 & 7.1 \\
\hline 3 days per week & 33 & 9.4 & 33 & 9.3 & 34 & 9.8 & 32 & 8.9 & 66 & 9.3 \\
\hline 2 days per week & 27 & 7.7 & 19 & 5.3 & 20 & 5.7 & 26 & 7.2 & 46 & 6.5 \\
\hline 1 day per week & 13 & 3.7 & 11 & 3.1 & 13 & 3.7 & 11 & 3.1 & 24 & 3.4 \\
\hline None & 101 & 28.7 & 117 & 32.9 & 116 & 33.3 & 102 & 28.3 & 218 & 30.8 \\
\hline Missing & 76 & 21.6 & 65 & 18.3 & 79 & 22.7 & 62 & 17.2 & 141 & 19.9 \\
\hline
\end{tabular}


TABLE 39 Participant-reported exercise adherence (continued)

\begin{tabular}{|c|c|c|c|c|c|c|c|c|c|c|}
\hline \multirow[b]{2}{*}{ Adherence } & \multicolumn{2}{|c|}{ Best-practice advice } & \multicolumn{2}{|c|}{ Progressive exercise } & \multicolumn{2}{|c|}{ No injection } & \multicolumn{2}{|c|}{ Injection } & \multicolumn{2}{|c|}{ Overall } \\
\hline & $n$ & $\%$ & $n$ & $\%$ & $n$ & $\%$ & $\mathbf{n}$ & $\%$ & $n$ & $\%$ \\
\hline \multicolumn{11}{|l|}{12 months } \\
\hline Every day & 15 & 4.3 & 16 & 4.5 & 9 & 2.6 & 22 & 6.1 & 31 & 4.4 \\
\hline 6 days per week & 3 & 0.9 & 1 & 0.3 & 3 & 0.9 & 1 & 0.3 & 4 & 0.6 \\
\hline 5 days per week & 22 & 6.3 & 15 & 4.2 & 13 & 3.7 & 24 & 6.7 & 37 & 5.2 \\
\hline 4 days per week & 16 & 4.5 & 12 & 3.4 & 14 & 4.0 & 14 & 3.9 & 28 & 4.0 \\
\hline 3 days per week & 24 & 6.8 & 26 & 7.3 & 21 & 6.0 & 29 & 8.1 & 50 & 7.1 \\
\hline 2 days per week & 19 & 5.4 & 22 & 6.2 & 17 & 4.9 & 24 & 6.7 & 41 & 5.8 \\
\hline 1 day per week & 18 & 5.1 & 16 & 4.5 & 21 & 6.0 & 13 & 3.6 & 34 & 4.8 \\
\hline None & 165 & 46.9 & 183 & 51.4 & 176 & 50.6 & 172 & 47.8 & 348 & 49.2 \\
\hline Missing & 69 & 19.6 & 64 & 18.0 & 74 & 21.3 & 59 & 16.4 & 133 & 18.8 \\
\hline
\end{tabular}

TABLE 40 Progressive exercise vs. best-practice advice and injection vs. best-practice advice: inside-the-table analysis of SPADI overall

\begin{tabular}{|c|c|c|c|c|}
\hline \multirow{2}{*}{$\begin{array}{l}\text { SPADI over } 12 \text { months } \\
\text { Unadjusted mean (SD), } n^{\text {a }}\end{array}$} & \multicolumn{2}{|c|}{$\begin{array}{l}\text { Progressive exercise }(N=174) \text { vs. } \\
\text { best-practice advice }(N=174)\end{array}$} & \multicolumn{2}{|c|}{$\begin{array}{l}\text { Injection plus best-practice advice } \\
(N=178) \text { vs. best-practice advice } \\
(N=174)\end{array}$} \\
\hline & 27.36 (23.49), 166 & 32.21 (25.39), 164 & 28.55 (23.11), 175 & 32.21 (25.39), 164 \\
\hline Adjusted mean (SE), $n^{\mathrm{b}}$ & 28.79 (1.39), 154 & 30.55 (1.43), 146 & 28.32 (1.41), 151 & 30.55 (1.43), 146 \\
\hline Unadjusted difference $(99 \% \mathrm{Cl})^{\mathrm{a}}$ & \multicolumn{2}{|c|}{$-4.64(-10.20$ to 0.91$)$} & \multicolumn{2}{|c|}{$-3.50(-8.99$ to 1.99$)$} \\
\hline $\begin{array}{l}\text { Adjusted difference }(99 \% \mathrm{Cl}) \text {, } \\
p \text {-value }\end{array}$ & \multicolumn{2}{|c|}{$-1.76(-6.91$ to 3.39$), 0.380$} & \multicolumn{2}{|c|}{$-2.23(-7.03$ to 2.57$), 0.230$} \\
\hline
\end{tabular}

a Unadjusted SPADI analysis using a mixed-effects model with random effects for observations within participant, with time-by-treatment interaction. A total of 1870 participant data points contribute to the unadjusted model.

b SPADI-adjusted analysis using a mixed-effects model with fixed effects for age, sex and baseline SPADI, and random effects for observations within participant, physiotherapist and centre, with time-by-treatment interaction. A total of 1869 participant data points contribute to the adjusted model. 
TABLE 41 Progressive exercise vs. best-practice advice and injection vs. best-practice advice: inside-the-table analysis of SPADI at each time point

\begin{tabular}{|c|c|c|c|c|c|c|c|c|}
\hline \multirow[b]{2}{*}{$\begin{array}{l}\text { SPADI at each } \\
\text { time point }\end{array}$} & \multicolumn{4}{|c|}{ Progressive exercise vs. best-practice advice } & \multicolumn{4}{|c|}{ Injection vs. best-practice advice } \\
\hline & $\begin{array}{l}\text { Adjusted mean } \\
\text { (SE), } n\end{array}$ & $\begin{array}{l}\text { Adjusted mean } \\
\text { (SE), } n\end{array}$ & $\begin{array}{l}\text { Adjusted difference } \\
(99 \% \mathrm{CI})^{\mathrm{a}}\end{array}$ & $p$-value & $\begin{array}{l}\text { Adjusted mean } \\
\text { (SE), } n\end{array}$ & $\begin{array}{l}\text { Adjusted mean } \\
\text { (SE), } n\end{array}$ & $\begin{array}{l}\text { Adjusted difference } \\
(99 \% \mathrm{CI})^{\mathrm{a}}\end{array}$ & $p$-value \\
\hline 8 weeks & 41.22 (1.78), 156 & 41.09 (1.72), 149 & $-0.13(-6.52$ to 6.27$)$ & 0.959 & 32.89 (1.75), 163 & 37.97 (1.68), 149 & $-8.33(-14.46$ to -2.19$)$ & 0.000 \\
\hline 6 months & 26.99 (1.81), 151 & 25.71 (1.74), 143 & $-1.28(-7.76$ to 5.20$)$ & 0.611 & 27.75 (1.76), 158 & 26.02 (1.70), 143 & $0.76(-5.45$ to 6.97$)$ & 0.752 \\
\hline 12 months & 23.12 (1.81), 153 & 19.19 (1.74), 143 & $-3.93(-10.40$ to 2.55$)$ & 0.118 & 24.17 (1.76), 160 & 21.90 (1.71), 143 & $1.05(-5.15$ to 7.26$)$ & 0.663 \\
\hline
\end{tabular}

a SPADI-adjusted analysis using a mixed-effects model with fixed effects for age, sex and baseline SPADI, and random effects for observations within participant, physiotherapist and centre, with time-by-treatment interaction. A total of 1869 participant data points contribute to the adjusted model. 
TABLE 42 Secondary outcomes analysis: unadjusted mean and SD

\begin{tabular}{|c|c|c|c|c|c|c|}
\hline \multirow[b]{2}{*}{ Secondary outcome } & Best-practice advice & Progressive exercise & \multicolumn{2}{|c|}{ No injection } & \multicolumn{2}{|c|}{ Injection } \\
\hline & $\begin{array}{l}\text { Unadjusted } \\
\text { mean (SD) }\end{array}$ & $\begin{array}{l}\text { Unadjusted } \\
\text { mean (SD) }\end{array}$ & $\boldsymbol{n}$ & $\begin{array}{l}\text { Unadjusted } \\
\text { mean (SD) }\end{array}$ & $\boldsymbol{n}$ & $\begin{array}{l}\text { Unadjusted } \\
\text { mean (SD) }\end{array}$ \\
\hline
\end{tabular}

SPADI pain

Baseline

8 weeks

$352 \quad 65.1(18) \quad 354$

$62.7(17.4)$

$346 \quad 63.3(17.6) \quad 360$

$64.4(17.9)$

6 months

314

$46.0(24.5)$

326

$46.7(24.5)$

$306 \quad 50.2(23.4)$

334

$42.7(24.9)$

12 months

$30134.4(26.4)$

314

$30.9(25.7)$

$29431.7(25.6)$

$32133.5(26.5)$

Over 12 months

304

$28.7(27.1)$

314

$24.6(25.5)$

$296 \quad 25.5(25.9)$

$322 \quad 27.7(26.8)$

$339 \quad 36.6(27.0)$

$343 \quad 34.2(26.9)$

$339 \quad 35.9(27.1) \quad 343 \quad 34.7(26.8)$

SPADI function

Baseline

8 weeks

352

47.2 (22.6)

354

41.5 (21.3)

$346 \quad 44.2(22.4)$

36044.5 (21.9)

6 months

313

$31.3(23.6)$

326

30.8 (23.5)

$307 \quad 33.3(23.5)$

$332 \quad 29.0(23.3)$

12 months

303

22.0 (22.6)

314

19.9 (22.1)

$294 \quad 20.7$ (22.6)

$32121.2(22.2)$

Over 12 months

339

$19.4(22.6)$

314

15.1 (21)

$296 \quad 16.3$ (21.8)

$321 \quad 18.0(22.1)$

FABQ-PA

Baseline

34

$24.3(23.5)$

343

22.0 (23.2)

$339 \quad 23.6(23.7)$

$343 \quad 22.8(23.0)$

8 weeks

6 months

279

$15.6(5.6)$

354

14.5 (5.4)

$344 \quad 14.9(5.7)$

359

$15.2(5.3)$

276

11.9 (6.4)

307

$11.9(6.0)$

$276 \quad 12.0(6.3)$

$310 \quad 11.8$ (6.1)

12 months

280

$9.9(6.5)$

287

$9.4(6.4)$

$2659.4(6.6)$

$298 \quad 9.8(6.3)$

Over 12 months

$324 \quad 10.4(6.6)$

8.1 (6.8)

$271 \quad 8.5(6.9)$

300

9.1 (6.5)

$316 \quad 10.0(6.8)$

$340 \quad 10.2$ (6.4)

PSEQ-2

Baseline

8 weeks

6 months

$352 \quad 9.6(2.4)$

354

$9.8(2.3)$

$346 \quad 9.7(2.3)$

$360 \quad 9.6(2.3)$

$\begin{array}{llll}280 & 10.2(2.4) \quad 306 & 10.3(2.2)\end{array}$

$276 \quad 10.2(2.3)$

$31010.3(2.3)$

12 months

276

$10.5(2.1)$

288

$10.4(2.2)$

26610.5 (2.1)

$29810.4(2.2)$

$280 \quad 10.7(2.0)$

$291 \quad 10.8(2.2)$

$271 \quad 10.9(1.8)$

$300 \quad 10.6(2.3)$

Over 12 months

$325 \quad 10.5(2.2)$

$332 \quad 10.5(2.2)$

$317 \quad 10.5$ (2.1)

$340 \quad 10.4(2.3)$

ISI

Baseline

$349 \quad 10.7$ (6.4)

$350 \quad 10.3(6.1)$

$343 \quad 10.2(6.3)$

356

$10.7(6.3)$

8 weeks

277

7.5 (6.2)

$303 \quad 8.0(6.1)$

$274 \quad 8.7(6.3)$

306

$7.0(6.0)$

6 months

$275 \quad 6.1(5.8)$

290

$6.2(6.1)$

$267 \quad 6.1(5.9)$

298

$6.2(6.0)$

12 months

281

5.8 (6.1)

292

$5.2(5.7)$

$272 \quad 5.4(5.9)$

301

$5.6(6.0)$

Over 12 months

$323 \quad 6.5(6.1)$

$329 \quad 6.5(6.1)$

$314 \quad 6.7(6.2)$

$338 \quad 6.3(6.0)$

RDA

\begin{tabular}{lllllllll} 
Baseline & 352 & $8.1(2.6)$ & 354 & $7.7(2.6)$ & 346 & $7.8(2.7)$ & 360 & $7.9(2.5)$ \\
8 weeks & 278 & $6.2(2.5)$ & 303 & $6.2(2.5)$ & 274 & $6.5(2.6)$ & 307 & $5.9(2.4)$ \\
6 months & 276 & $5.4(2.4)$ & 290 & $5.0(2.3)$ & 268 & $5.1(2.4)$ & 298 & $5.2(2.3)$ \\
12 months & 281 & $4.8(2.3)$ & 292 & $4.6(2.3)$ & 273 & $4.6(2.4)$ & 300 & $4.8(2.3)$ \\
Over 12 months & 325 & $5.5(2.5)$ & 332 & $5.3(2.5)$ & 317 & $5.4(2.6)$ & 340 & $5.3(2.4)$ \\
\hline
\end{tabular}


TABLE 42 Secondary outcomes analysis: unadjusted mean and SD (continued)

\begin{tabular}{|c|c|c|c|c|c|c|c|c|}
\hline \multirow[b]{2}{*}{ Secondary outcome } & \multicolumn{2}{|c|}{ Best-practice advice } & \multicolumn{2}{|c|}{ Progressive exercise } & \multicolumn{2}{|c|}{ No injection } & \multicolumn{2}{|c|}{ Injection } \\
\hline & $n$ & $\begin{array}{l}\text { Unadjusted } \\
\text { mean (SD) }\end{array}$ & $n$ & $\begin{array}{l}\text { Unadjusted } \\
\text { mean (SD) }\end{array}$ & $n$ & $\begin{array}{l}\text { Unadjusted } \\
\text { mean (SD) }\end{array}$ & $n$ & $\begin{array}{l}\text { Unadjusted } \\
\text { mean (SD) }\end{array}$ \\
\hline \multicolumn{9}{|l|}{ GIT } \\
\hline \multicolumn{9}{|l|}{ Baseline } \\
\hline 8 weeks & 277 & $7.6(2.1)$ & 304 & $7.8(1.8)$ & 274 & $7.3(2.0)$ & 307 & $8.1(1.8)$ \\
\hline 6 months & 276 & $8.2(2.2)$ & 291 & $8.7(2.1)$ & 269 & $8.5(2.2)$ & 298 & $8.5(2.2)$ \\
\hline 12 months & 283 & $8.6(2.3)$ & 293 & $9.1(2.2)$ & 274 & $8.9(2.1)$ & 302 & $8.8(2.4)$ \\
\hline Over 12 months & 326 & $8.2(2.2)$ & 332 & $8.5(2.1)$ & 317 & $8.2(2.2)$ & 347 & $8.5(2.1)$ \\
\hline
\end{tabular}

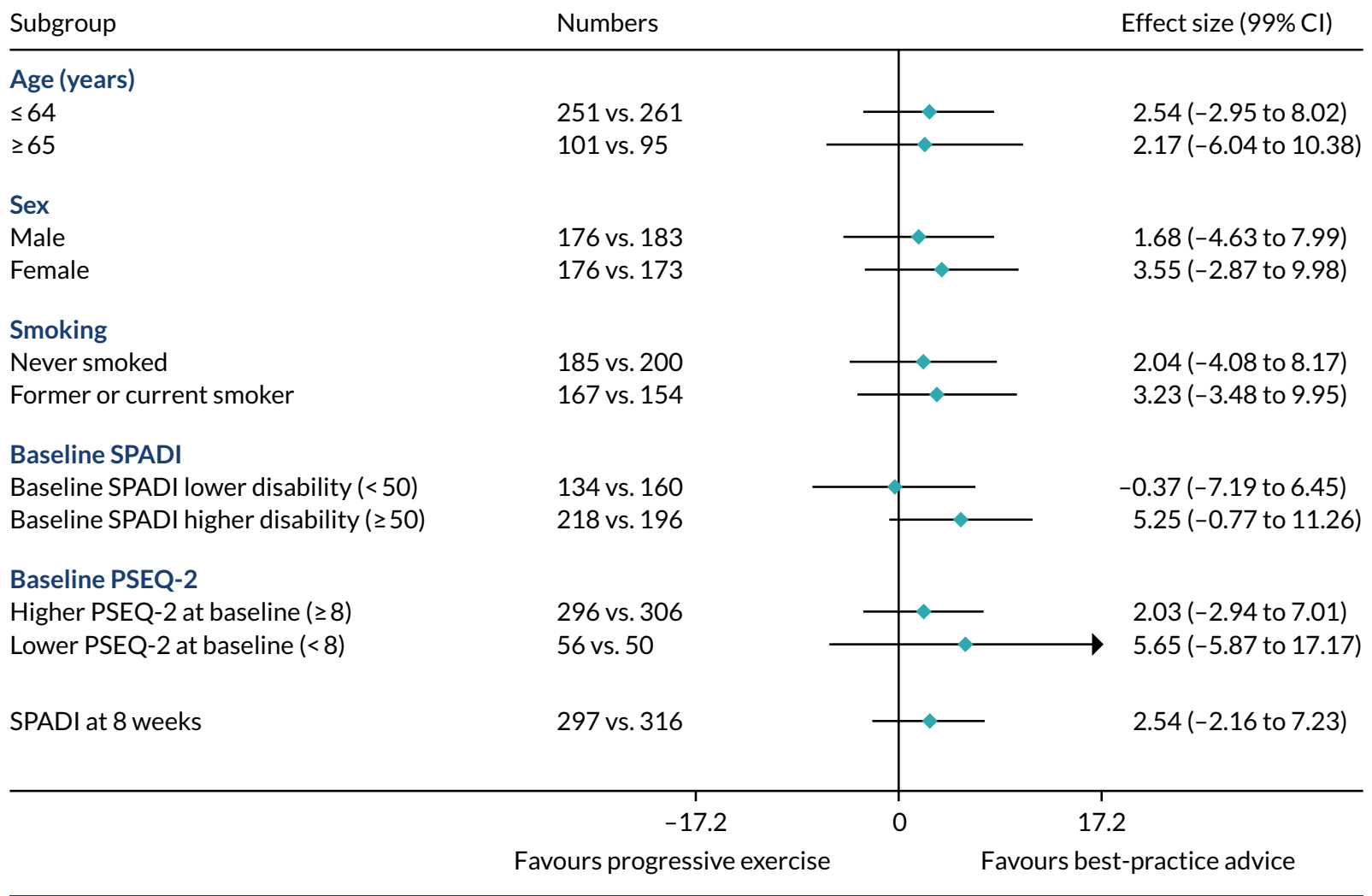

FIGURE 25 Subgroup-adjusted SPADI analysis for progressive exercise vs. best-practice advice at 8 weeks. 


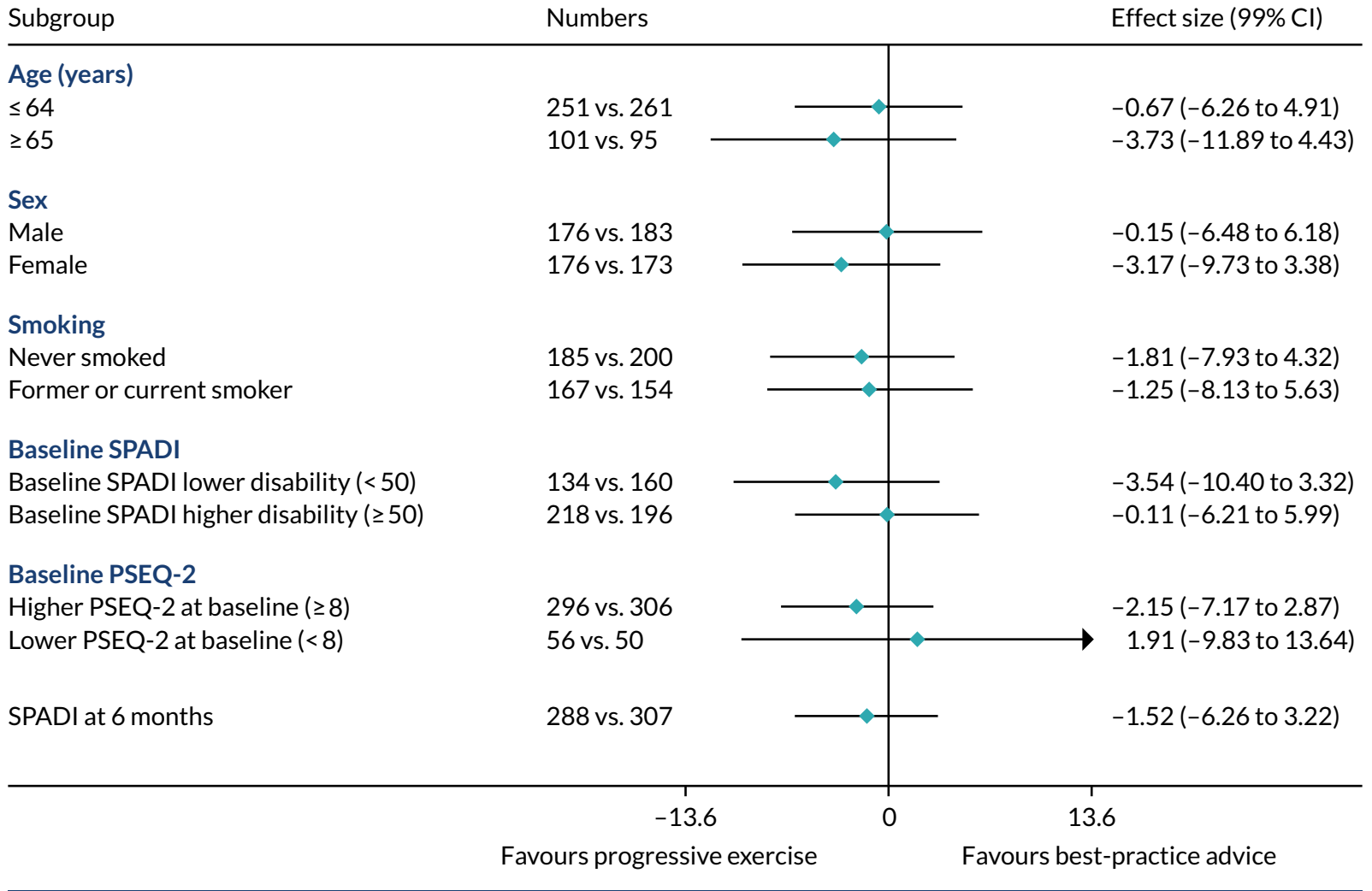

FIGURE 26 Subgroup-adjusted SPADI analysis for progressive exercise vs. best-practice advice at 6 months.

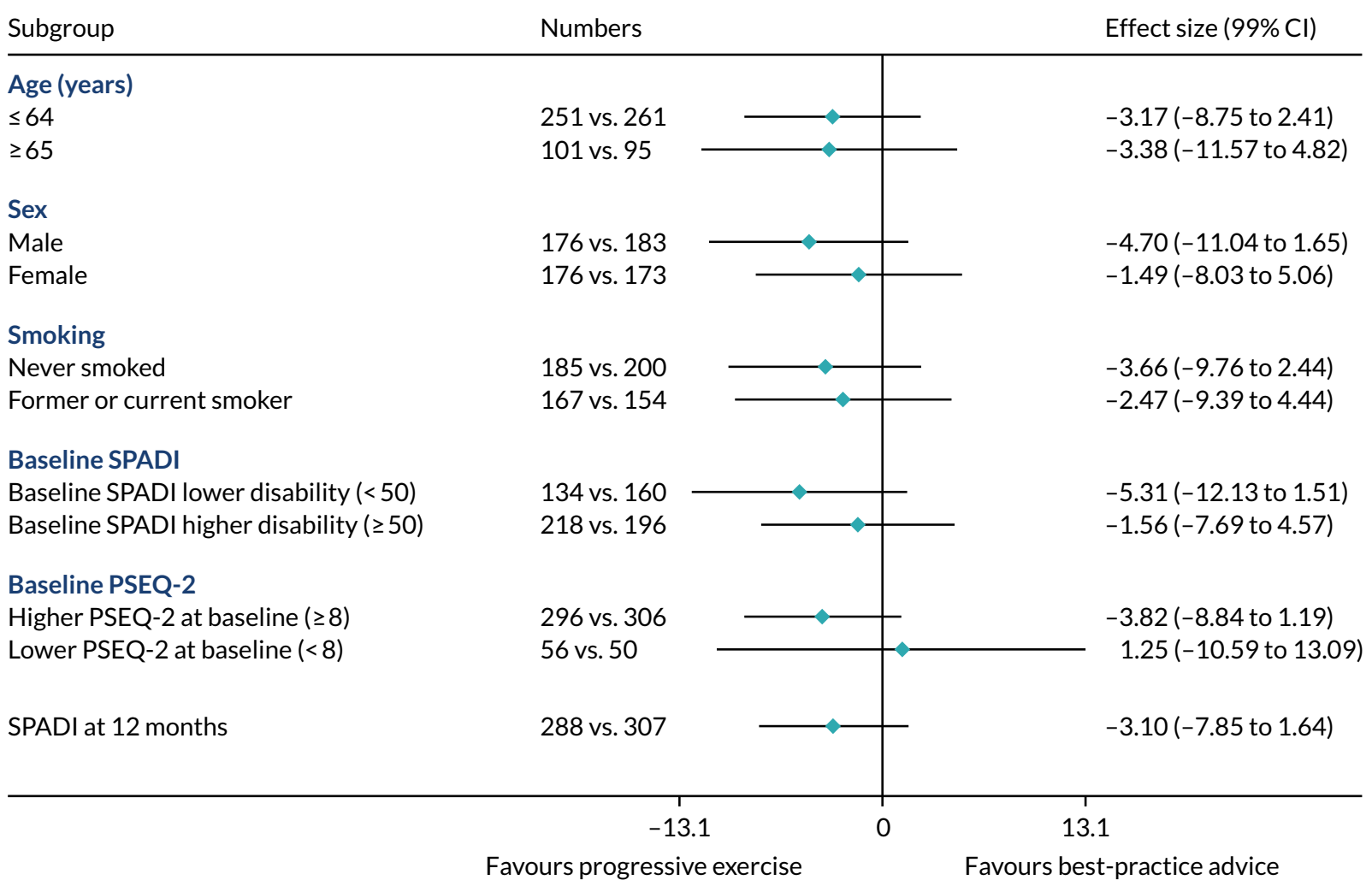

FIGURE 27 Subgroup-adjusted SPADI analysis for progressive exercise vs. best-practice advice at 12 months. 


\begin{tabular}{|c|c|c|}
\hline Subgroup & Numbers & Effect size $(99 \% \mathrm{Cl})$ \\
\hline \multicolumn{3}{|l|}{ Age (years) } \\
\hline$\leq 64$ & $\longrightarrow$ & $-7.14(-12.23$ to -2.05$)$ \\
\hline$\geq 65$ & 98 vs. 98 & $-1.81(-9.77$ to 6.16$)$ \\
\hline \multicolumn{3}{|l|}{ Sex } \\
\hline Male & 177 vs. 182 & $-4.41(-10.43$ to 1.61$)$ \\
\hline Female & $\longrightarrow$ & $-7.03(-13.14$ to -0.92$)$ \\
\hline \multicolumn{3}{|l|}{ Smoking } \\
\hline Never smoked & 184 vs. 201 & $-5.50(-11.28$ to 0.29$)$ \\
\hline Former or current smoker & 162 vs. 159 & $-5.73(-12.16$ to 0.70$)$ \\
\hline \multicolumn{3}{|l|}{ Baseline SPADI } \\
\hline Baseline SPADI lower disability $(<50)$ & 144 vs. 150 & $-0.36(-6.87$ to 6.16$)$ \\
\hline Baseline SPADI higher disability $(\geq 50)$ & 204 vs. 210 & $-9.67(-15.37$ to -3.97$)$ \\
\hline \multicolumn{3}{|l|}{ Baseline PSEQ-2 } \\
\hline Higher PSEQ-2 at baseline $(\geq 8)$ & 297 vs. 305 & $-5.42(-10.05$ to -0.79$)$ \\
\hline Lower PSEQ-2 at baseline $(<8)$ & 51 vs. 55 & $-7.32(-18.64$ to 4.01$)$ \\
\hline \multirow[t]{3}{*}{ SPADI at 8 weeks } & 300 vs. 313 & $-5.64(-9.93$ to -1.35$)$ \\
\hline & -18.6 & 18.6 \\
\hline & Favours injection & Favours no injection \\
\hline
\end{tabular}

FIGURE 28 Subgroup-adjusted SPADI analysis for injection vs. no injection at 8 weeks.



FIGURE 29 Subgroup-adjusted SPADI analysis for injection vs. no injection at 6 months. 


\begin{tabular}{|c|c|c|c|}
\hline Subgroup & Numbers & & Effect size $(99 \% \mathrm{Cl})$ \\
\hline \multicolumn{4}{|l|}{ Age (years) } \\
\hline$\leq 64$ & 250 vs. 262 & $-\infty$ & $1.49(-3.69$ to 6.66$)$ \\
\hline$\geq 65$ & 98 vs. 98 & $\longrightarrow$ & $3.33(-4.62$ to 11.28$)$ \\
\hline \multicolumn{4}{|l|}{ Sex } \\
\hline Male & 177 vs. 182 & $-\backsim$ & $2.95(-3.09$ to 8.99$)$ \\
\hline Female & 171 vs. 178 & 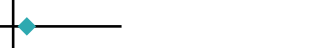 & $0.86(-5.37$ to 7.09$)$ \\
\hline \multicolumn{4}{|l|}{ Smoking } \\
\hline Never smoked & 184 vs. 201 & & $-0.28(-6.03$ to 5.48$)$ \\
\hline Former or current smoker & 162 vs. 159 & - & $4.87(-1.77$ to 11.51$)$ \\
\hline \multicolumn{4}{|l|}{ Baseline SPADI } \\
\hline Baseline SPADI lower disability $(<50)$ & 144 vs. 150 & - & $4.23(-2.29$ to 10.75$)$ \\
\hline Baseline SPADI higher disability $(\geq 50)$ & 204 vs. 210 & & $0.14(-5.68$ to 5.95$)$ \\
\hline \multicolumn{4}{|l|}{ Baseline PSEQ-2 } \\
\hline Higher PSEQ- 2 at baseline $(\geq 8)$ & 297 vs. 305 & - & $1.14(-3.53$ to 5.81$)$ \\
\hline Lower PSEQ-2 at baseline $(<8)$ & 51 vs. 55 & & $6.80(-4.84$ to 18.44$)$ \\
\hline \multirow[t]{3}{*}{ SPADI at 12 months } & 296 vs. 321 & $-\backsim$ & $1.93(-2.41$ to 6.27$)$ \\
\hline & $\begin{array}{c}18.4 \\
-18.4\end{array}$ & 18.4 & \\
\hline & Favours injection & Favours no injection & \\
\hline
\end{tabular}

FIGURE 30 Subgroup-adjusted SPADI analysis for injection vs. no injection at 12 months. 


\section{Appendix 5 Chapter 5 appendix}

TABLE 43 Summary of prescribed medication unit cost (in 2019 £)

\begin{tabular}{|c|c|c|c|}
\hline Medication & Unit type & Unit cost (£) & Source \\
\hline \multicolumn{4}{|l|}{ Analgesics } \\
\hline Amitriptyline (10 mg) & Pack of 28 & 1.01 & NHS Electronic Drug Tariff ${ }^{127}$ \\
\hline Co-codamol (30 mg/500 mg) & Pack of 100 & 4.60 & NHS Electronic Drug Tariff ${ }^{127}$ \\
\hline Solpadol (30 mg) & Pack of 100 & 5.28 & NHS Electronic Drug Tariff ${ }^{127}$ \\
\hline Codeine (60 mg) & Pack of 28 & 1.89 & NHS Electronic Drug Tariff ${ }^{127}$ \\
\hline Co-dydramol (10 mg) & Pack of 30 & 0.93 & NHS Electronic Drug Tariff ${ }^{127}$ \\
\hline Tramadol (50 mg) & Pack of 30 & 0.85 & NHS Electronic Drug Tariff ${ }^{127}$ \\
\hline Paracetamol (500 mg) & Pack of 100 & 2.28 & NHS Electronic Drug Tariff ${ }^{127}$ \\
\hline $\begin{array}{l}\text { Morphine sulphate (Sevredol }{ }^{\circledR}, \text { Napp } \\
\text { Pharmaceuticals Limited, Cambridge, UK) } \\
\text { (10 mg) }\end{array}$ & Pack of 56 & 5.31 & NHS Electronic Drug Tariff ${ }^{127}$ \\
\hline Celecoxib (100 mg) & Pack of 60 & 2.30 & NHS Electronic Drug Tariff ${ }^{127}$ \\
\hline Gabapentin $(600 \mathrm{mg})$ & Pack of 100 & 12.00 & NHS Electronic Drug Tariff ${ }^{127}$ \\
\hline \multicolumn{4}{|l|}{ NSAIDs } \\
\hline Ibuprofen (200 mg) & Pack of 16 & 2.67 & NHS Electronic Drug Tariff ${ }^{127}$ \\
\hline Ibuprofen gel & Each & 2.10 & NHS Electronic Drug Tariff ${ }^{127}$ \\
\hline Meloxicam (15 mg) & Pack of 30 & 1.77 & NHS Electronic Drug Tariff ${ }^{127}$ \\
\hline Naproxen (500 mg) & Pack of 56 & 4.14 & NHS Electronic Drug Tariff ${ }^{127}$ \\
\hline Diclofenac (25 mg) & Pack of 28 & 3.86 & NHS Electronic Drug Tariff ${ }^{127}$ \\
\hline \multicolumn{4}{|l|}{ Other medication usage } \\
\hline Phorpain & Each & 2.72 & NHS Electronic Drug Tariff ${ }^{127}$ \\
\hline Temazepam (20 mg) & Pack of 28 & 1.60 & NHS Electronic Drug Tariff ${ }^{127}$ \\
\hline Vitamin D & Pack of 30 & 4.93 & NHS Electronic Drug Tariff ${ }^{127}$ \\
\hline Voltarol emulgel & Each & 2.04 & NHS Electronic Drug Tariff ${ }^{127}$ \\
\hline Zapain (30 mg) & Pack of 100 & 4.77 & NHS Electronic Drug Tariff ${ }^{127}$ \\
\hline Diazepan (2 mg) & Pack of 28 & 0.76 & NHS Electronic Drug Tariff ${ }^{127}$ \\
\hline Movelat gel & Each & 8.49 & NHS Electronic Drug Tariff ${ }^{127}$ \\
\hline Adcal (750 mg) & Pack of 112 & 2.95 & NHS Electronic Drug Tariff ${ }^{127}$ \\
\hline Alendronic (10 mg) & Pack of 28 & 2.63 & NHS Electronic Drug Tariff ${ }^{127}$ \\
\hline Prednisolone (1 mg) & Pack of 28 & 0.85 & NHS Electronic Drug Tariff ${ }^{127}$ \\
\hline Feldene gel (60 g) & Each & 6.00 & NHS Electronic Drug Tariff ${ }^{127}$ \\
\hline Sertraline (100 mg) & Pack of 28 & 3.82 & NHS Electronic Drug Tariff ${ }^{127}$ \\
\hline
\end{tabular}


TABLE 43 Summary of prescribed medication unit cost (in 2019 f) (continued)

\begin{tabular}{|c|c|c|c|}
\hline Medication & Unit type & Unit cost (£) & Source \\
\hline Docycycline $(50 \mathrm{mg})$ & Pack of eight & 2.25 & NHS Electronic Drug Tariff ${ }^{127}$ \\
\hline Butrans patch $(5 \mu \mathrm{g})$ & Pack of four & 17.60 & NHS Electronic Drug Tariff ${ }^{127}$ \\
\hline \multicolumn{4}{|l|}{ Corticosteroid injections } \\
\hline Kenalog (40 mg) & Each & 7.45 & NHS Electronic Drug Tariff ${ }^{127}$ \\
\hline Depomedrone (40 mg) & Each & 7.13 & NHS Electronic Drug Tariff ${ }^{127}$ \\
\hline \multicolumn{4}{|l|}{ Local anaesthetic } \\
\hline Lidocaine $(50 \mathrm{mg} / 5 \mathrm{ml})(1 \%)$ & Pack of 20 & 6.50 & NHS Electronic Drug Tariff ${ }^{127}$ \\
\hline Bupivacaine hydrochloride $(50 \mathrm{mg} / 10 \mathrm{ml})(0.5 \%)$ & Pack of 10 & 7.56 & NHS Electronic Drug Tariff ${ }^{127}$ \\
\hline
\end{tabular}

TABLE 44 Unit costs of health and social care items and additional financial cost due to rotator cuff disorder

\begin{tabular}{|c|c|c|c|}
\hline Resource item & Unit & Unit cost $(\boldsymbol{E})^{\mathrm{a}}$ & Source \\
\hline \multicolumn{4}{|l|}{ Inpatient stay (because of shoulder) } \\
\hline Surgery to repair rotator cuff tear & Episode & 3711.07 & $\begin{array}{l}\text { NHS Reference Costs 2017:92 } \\
\text { elective HRG HN53A, } \\
\text { HN53B, HN53C }\end{array}$ \\
\hline Frozen shoulder surgery & Episode & 3064.07 & $\begin{array}{l}\text { NHS Reference Costs 2017:92 } \\
\text { elective HRG HN54A, } \\
\text { HN54B, HN54C }\end{array}$ \\
\hline Subacromial decompression and cuff repair & Episode & 3711.07 & $\begin{array}{l}\text { NHS Reference Costs 2017:92 } \\
\text { elective HRG HN53A, } \\
\text { HN53B, HN53C }\end{array}$ \\
\hline \multicolumn{4}{|l|}{ Outpatient care } \\
\hline Orthopaedic clinic (shoulder) & Visit & 120.00 & $\begin{array}{l}\text { NHS Reference Costs 2019:128 } \\
110\end{array}$ \\
\hline Physiotherapy & Visit & 38.88 & PSSRU 2019:89 p. 68 \\
\hline Radiology (X-rays) & Test & 31.00 & $\begin{array}{l}\text { NHS Reference Costs 2017:92 } \\
\text { DAPF }\end{array}$ \\
\hline Radiology (ultrasound) & Test & 39.00 & $\begin{array}{l}\text { NHS Reference Costs 2019:128 } \\
\text { RD40Z }\end{array}$ \\
\hline Radiology (MRI) & Test & 108.00 & $\begin{array}{l}\text { NHS Reference Costs 2019:128 } \\
\text { RD01A }\end{array}$ \\
\hline Emergency department & Visit & 106.00 & $\begin{array}{l}\text { NHS Reference Costs 2019:128 } \\
\text { VB09Z }\end{array}$ \\
\hline \multicolumn{4}{|l|}{ Community care (NHS) } \\
\hline GP (surgery) & 9.22-minute visit & 39.23 & PSSRU 2019:89 p. 120 \\
\hline GP (home) & Per minute & 5.32 & PSSRU 2010:129 p. 167 \\
\hline
\end{tabular}


TABLE 44 Unit costs of health and social care items and additional financial cost due to rotator cuff disorder (continued)

\begin{tabular}{|c|c|c|c|}
\hline Resource item & Unit & Unit cost $(\boldsymbol{E})^{\mathrm{a}}$ & Source \\
\hline GP (telephone contact) & 7.1 minutes & 27.62 & PSSRU 2015:130 p. 177 \\
\hline Practice nurse & Hour visit & 42.00 & PSSRU 2019:89 p. 118 \\
\hline 111 advice & Per call & 14.32 & Financial Times $2017^{131}$ \\
\hline Physiotherapist & Session & 36.83 & PSSRU 2019:89 p. 82 \\
\hline \multicolumn{4}{|l|}{ Community care (private) } \\
\hline Orthopaedic clinic (shoulder) & Visit & 200.00 & Orthopaedic Clinics UK ${ }^{132}$ \\
\hline Physiotherapy & Visit & 75.00 & The Physiotherapy Centre ${ }^{133}$ \\
\hline Radiology (X-rays) & Test & 90.00 & Orthopaedic Clinics UK ${ }^{132}$ \\
\hline Radiology (ultrasound) & Test & 358.00 & Private Healthcare UK ${ }^{134}$ \\
\hline Radiology (MRI) & Test & 285.00 & Orthopaedic Clinics UK ${ }^{132}$ \\
\hline Chiropractor & Visit & 35.00 & Orthopaedic Clinics UK ${ }^{132}$ \\
\hline $\begin{array}{l}\text { Complementary therapist } \\
\text { (e.g. reflexology, acupuncture) }\end{array}$ & Visit & 65.00 & Orthopaedic Clinics UK ${ }^{132}$ \\
\hline Sports massage therapist & Visit & 60.00 & Orthopaedic Clinics UK ${ }^{132}$ \\
\hline
\end{tabular}

Direct non-medical cost

Help with housework

Trial

Help with childcare

Trial

Travel

Trial

Lost productivity

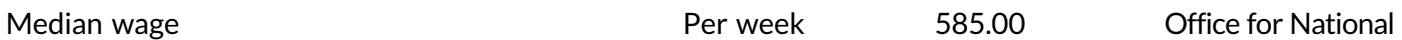
Statistics ${ }^{135}$

HRG, Healthcare Resource Group; MRI, magnetic resonance imaging; PSSRU, Personal Social Service Research Unit. a Unit cost has been inflated to $2018 / 19$ prices.

Note

If more than one code applies, costs are estimated weighted by activity as depicted in annex A (Department of Health and Social Care). ${ }^{136}$

\section{Model 1: regression analysis model with interaction term (base-case analysis)}

For the $2 \times 2$ GRASP factorial trial, the regression model predicting costs with an interaction term took the form:

$$
y_{i}=\beta_{0}+\beta_{A} A_{i}+\beta_{B} B_{i}+\beta_{A B} A_{i} B_{i}+\varepsilon_{i}
$$

where $y_{i}$ represents the outcome measure (cost or QALY), $\beta_{A}$ represents the coefficient for treatment effects for exercise, $\beta_{B}$ represents the coefficient for treatment effects for the corticosteroid injection, $\beta_{A B}$ represents the interaction coefficient between exercise and corticosteroid injection and $\beta_{o}$ represents the constant term.

Note that the QALY equation included baseline utility. 


\section{Model 2: regression analysis without interaction term (sensitivity analysis)}

This analysis was used as a sensitivity analysis. This analysis was carried out by analysing the same set of bootstraps, omitting the interaction between injection and progressive exercise, and results were combined across bootstraps and imputations using the same methods as the base-case analysis. This approach assumes that interventions are mutually exclusive. In other words, the cost and outcomes of the individually tailored progressive-exercise programme are assumed to not be affected by whether or not corticosteroid injection is given (and vice versa). In addition, regression analysis without interaction term, although similar to the at-the-margins approach, makes the prediction of group means and SEs easier. As interventions are assumed to be mutually exclusive options, these predictions can help us identify which of the four treatment options (progressive exercise, best-practice advice, corticosteroid injection plus progressive exercise or corticosteroid injection plus best-practice advice) maximises NMB and also estimates the cost and effects of each option separately.

For the $2 \times 2$ GRASP factorial trial, the regression model for this sensitivity analysis took the form:

$$
y_{i}=\beta_{o}+\beta_{A} A_{i}+\beta_{B} B_{i}+\varepsilon_{i},
$$

where $y_{i}$ represents the outcome measure (cost, QALY or NMB), $\beta_{A}$ represents the coefficient for treatment effects for exercise, $\beta_{B}$ represents the coefficient for treatment effects for the corticosteroid injection and $\beta_{o}$ represents the constant term.

Note that the QALY equation included baseline utility. 
TABLE 45 Mean health resource utilisation: baseline to 8 weeks (available-case analysis, excluding patients with missing data on that resource)

\begin{tabular}{|c|c|c|c|c|c|c|c|c|c|c|c|c|}
\hline \multirow[b]{2}{*}{ Type of care } & \multicolumn{3}{|c|}{ Best-practice advice $(N=150)$} & \multicolumn{3}{|c|}{$\begin{array}{l}\text { Injection plus best-practice } \\
\text { advice }(N=165)\end{array}$} & \multicolumn{3}{|c|}{ Progressive exercise $(N=157)$} & \multicolumn{3}{|c|}{$\begin{array}{l}\text { Injection plus progressive } \\
\text { exercise }(N=169)\end{array}$} \\
\hline & $n^{\mathrm{a}}$ & $\begin{array}{l}\text { Completion } \\
\text { rate }^{\mathrm{b}}(\%)\end{array}$ & $\operatorname{Mean}^{c}$ (SD) & $n^{\mathrm{a}}$ & $\begin{array}{l}\text { Completion } \\
\text { rate }^{\mathrm{b}}(\%)\end{array}$ & Meanc (SD) $^{c}$ & $n^{a}$ & $\begin{array}{l}\text { Completion } \\
\text { rate }^{\mathrm{b}}(\%)\end{array}$ & 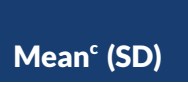 & $n^{\mathrm{a}}$ & $\begin{array}{l}\text { Completion } \\
\text { rate }^{\mathrm{b}}(\%)\end{array}$ & 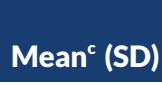 \\
\hline $\begin{array}{l}\text { Primary care (NHS community- } \\
\text { based services) }\end{array}$ & 129 & & & 150 & & & 146 & & & 162 & & \\
\hline GP visit & 127 & 98.45 & $0.15(0.47)$ & 149 & 99.33 & $0.06(0.29)$ & 138 & 94.52 & $0.13(0.42)$ & 157 & 96.91 & $0.04(0.19)$ \\
\hline GP telephone contact & 117 & 90.7 & $0.04(0.24)$ & 143 & 95.33 & $0.01(0.08)$ & 125 & 85.62 & $0.02(0.18)$ & 154 & 95.06 & $0.05(0.50)$ \\
\hline GP home visit & 113 & 87.6 & 0 & 142 & 94.67 & 0 & 124 & 84.93 & 0 & 152 & 93.83 & 0 \\
\hline Practice nurse & 114 & 88.37 & $0.02(0.19)$ & 142 & 94.67 & 0 & 125 & 85.62 & $0.04(0.45)$ & 154 & 95.06 & $0.01(0.11)$ \\
\hline $\begin{array}{l}\text { Physiotherapy (not as part } \\
\text { of the GRASP trial) }\end{array}$ & 114 & 88.37 & $0.05(0.56)$ & 143 & 95.33 & $0.001(0.08)$ & 132 & 90.41 & $0.121(0.51)$ & 154 & 95.06 & $0.02(0.14)$ \\
\hline $\begin{array}{l}\text { Other NHS community } \\
\text { services }\end{array}$ & 114 & 88.37 & 0 & 143 & 95.33 & 0 & 126 & 86.3 & $0.03(0.28)$ & 152 & 93.83 & $0.01(0.08)$ \\
\hline $\begin{array}{l}\text { Secondary care (NHS } \\
\text { outpatient services) }\end{array}$ & 129 & & & 150 & & & 146 & & & 163 & & \\
\hline $\begin{array}{l}\text { Orthopaedic clinic } \\
\text { (for shoulder) }\end{array}$ & 124 & 96.12 & $0.01(0.09)$ & 145 & 96.67 & $0.01(0.16)$ & 136 & 93.15 & 0 & 160 & 98.16 & 0 \\
\hline $\begin{array}{l}\text { Physiotherapy department } \\
\text { (not as part of the } \\
\text { GRASP trial) }\end{array}$ & 123 & 95.35 & 0 & 145 & 96.67 & $0.03(0.42)$ & 138 & 94.52 & $0.03(0.27)$ & 161 & 98.77 & $0.02(0.32)$ \\
\hline Radiology: X-ray & 125 & 99.21 & $0.02(0.13)$ & 147 & 98.66 & $0.02(0.14)$ & 144 & 100 & $0.08(0.34)$ & 160 & 98.77 & 0 \\
\hline Radiology: ultrasound & 124 & 98.41 & $0.01(0.09)$ & 147 & 98.66 & $0.02(0.14)$ & 136 & 94.44 & 0 & 162 & 100 & $0.01(0.11)$ \\
\hline Radiology: MRI & 123 & 97.62 & 0 & 144 & 96.64 & 0 & 136 & 94.44 & 0 & 160 & 98.77 & 0 \\
\hline Accident and emergency & 125 & 96.9 & $0.02(0.13)$ & 147 & 98 & $0.02(0.14)$ & 136 & 100 & 0 & 160 & 98.16 & 0 \\
\hline Other outpatient services & 123 & 95.35 & 0 & 146 & 97.33 & $0.01(0.12)$ & 136 & 100 & 0 & 160 & 98.16 & 0 \\
\hline $\begin{array}{l}\text { Secondary care (NHS inpatient } \\
\text { services) }\end{array}$ & 129 & & & 150 & & & 145 & & & 162 & & \\
\hline Inpatient care & 129 & 100 & 0 & 150 & 100 & 0 & 145 & 100 & 0 & 162 & 100 & 0 \\
\hline
\end{tabular}


TABLE 45 Mean health resource utilisation: baseline to 8 weeks (available-case analysis, excluding patients with missing data on that resource) (continued)

\begin{tabular}{|c|c|c|c|c|c|c|c|c|c|c|c|c|}
\hline \multirow[b]{2}{*}{ Type of care } & \multicolumn{3}{|c|}{ Best-practice advice $(N=150)$} & \multicolumn{3}{|c|}{$\begin{array}{l}\text { Injection plus best-practice } \\
\text { advice }(N=165)\end{array}$} & \multicolumn{3}{|c|}{ Progressive exercise $(\mathrm{N}=157)$} & \multicolumn{3}{|c|}{$\begin{array}{l}\text { Injection plus progressive } \\
\text { exercise }(N=169)\end{array}$} \\
\hline & $n^{a}$ & $\begin{array}{l}\text { Completion } \\
\text { rate }^{\mathrm{b}}(\%)\end{array}$ & 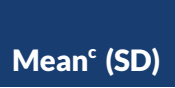 & $n^{a}$ & $\begin{array}{l}\text { Completion } \\
\text { rate }^{\mathrm{b}}(\%)\end{array}$ & Mean $^{c}$ (SD) & $n^{\mathrm{a}}$ & $\begin{array}{l}\text { Completion } \\
\text { rate }^{\mathrm{b}}(\%)\end{array}$ & Mean (SD) & $n^{a}$ & $\begin{array}{l}\text { Completion } \\
\text { rate }^{\mathrm{b}}(\%)\end{array}$ & $\operatorname{Mean}^{\mathrm{c}}$ (SD) \\
\hline Private care & 129 & & & 151 & & & 146 & & & 162 & & \\
\hline $\begin{array}{l}\text { Orthopaedic clinic } \\
\text { (for shoulder) }\end{array}$ & 127 & 98.45 & 0 & 150 & 100 & 0 & 144 & 98.63 & $0.01(0.08)$ & 162 & 100 & 0 \\
\hline Physiotherapy department & 128 & 73.56 & $0.03(0.35)$ & 150 & 100 & 0 & 143 & 97.95 & 0 & 162 & 100 & 0 \\
\hline Radiology: X-ray & 127 & 100 & 0 & 150 & 99.34 & 0 & 143 & 100 & 0 & 162 & 100 & 0 \\
\hline Radiology: ultrasound & 127 & 100 & 0 & 151 & 100 & 0 & 143 & 100 & 0 & 162 & 100 & 0 \\
\hline Radiology: MRI & 127 & 100 & 0 & 150 & 99.34 & 0 & 143 & 100 & 0 & 162 & 100 & 0 \\
\hline Chiropractor & 127 & 98.45 & 0 & 150 & 99.34 & 0 & 144 & 98.63 & $0.01(0.08)$ & 162 & 100 & 0 \\
\hline Complementary therapist & 128 & 99.22 & $0.05(0.53)$ & 150 & 99.34 & 0 & 144 & 98.63 & $0.01(0.17)$ & 162 & 100 & 0 \\
\hline Injection utilisation & 130 & & & 151 & & & 147 & & & 163 & & \\
\hline Injections & 130 & & $0.02(0.18)$ & 151 & & & 147 & & $0.02(0.14)$ & 163 & & $0.06(0.24)$ \\
\hline Non-medical expenses & 129 & & & 150 & & & 146 & & & 162 & & \\
\hline \multicolumn{13}{|c|}{$\begin{array}{l}\text { MRI, magnetic resonance imaging. } \\
\text { a Relates to the number of participants for whom follow-up data were available. } \\
\text { b Completion rate of the corresponding question related to usage or not of resource utilisation. } \\
\text { c Refers to mean resource utilisation per patient. }\end{array}$} \\
\hline
\end{tabular}


TABLE 46 Mean health resource utilisation: 8 weeks to 6 months (available-case analysis, excluding patients with missing data on that resource)

\begin{tabular}{|c|c|c|c|c|c|c|c|c|c|c|c|c|}
\hline \multirow[b]{2}{*}{ Type of care } & \multicolumn{3}{|c|}{ Best-practice advice $(N=144)$} & \multicolumn{3}{|c|}{$\begin{array}{l}\text { Injection plus best-practice } \\
\text { advice }(N=158)\end{array}$} & \multicolumn{3}{|c|}{ Progressive exercise $(\mathrm{N}=151)$} & \multicolumn{3}{|c|}{$\begin{array}{l}\text { Injection plus progressive } \\
\text { exercise }(N=162)\end{array}$} \\
\hline & $n^{\mathrm{a}}$ & $\begin{array}{l}\text { Completion } \\
\text { rate }^{\mathrm{b}}(\%)\end{array}$ & 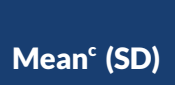 & $n^{a}$ & $\begin{array}{l}\text { Completion } \\
\text { rate }^{\mathrm{b}}(\%)\end{array}$ & Mean $^{c}$ (SD) & $n^{a}$ & $\begin{array}{l}\text { Completion } \\
\text { rate }^{\mathrm{b}}(\%)\end{array}$ & Mean $^{c}$ (SD) & $n^{\mathrm{a}}$ & $\begin{array}{l}\text { Completion } \\
\text { rate }^{\mathrm{b}}(\%)\end{array}$ & Meanc (SD) $^{c}$ \\
\hline $\begin{array}{l}\text { Primary care (NHS community- } \\
\text { based services) }\end{array}$ & 127 & & & 147 & & & 140 & & & 150 & & \\
\hline GP visit & 123 & 96.85 & $0.15(0.59)$ & 142 & 96.6 & $0.09(0.36)$ & 135 & 96.43 & $0.16(0.56)$ & 149 & 99.33 & $0.14(0.45)$ \\
\hline GP telephone contact & 117 & 92.13 & $0.08(0.44)$ & 133 & 90.48 & $0.01(0.09)$ & 123 & 87.86 & $0.02(0.18)$ & 135 & 90 & $0.01(0.09)$ \\
\hline GP home visit & 112 & 88.19 & 0 & 133 & 90.48 & 0 & 122 & 87.14 & 0 & 134 & 89.33 & 0 \\
\hline Practice nurse & 113 & 88.98 & $0.01(0.09)$ & 132 & 89.8 & 0 & 122 & 87.14 & 0 & 134 & 89.33 & 0 \\
\hline $\begin{array}{l}\text { Physiotherapy (not as part } \\
\text { of the GRASP trial) }\end{array}$ & 117 & 92.13 & $0.19(1.13)$ & 138 & 93.88 & $0.09(0.57)$ & 126 & 90 & $0.08(0.52)$ & 136 & 90.67 & $0.02(0.19)$ \\
\hline Other NHS community services & 113 & 88.98 & $0.02(0.02)$ & 132 & 89.8 & 0 & 123 & 87.86 & $0.03(0.36)$ & 136 & 90.67 & $0.01(0.12)$ \\
\hline $\begin{array}{l}\text { Secondary care (NHS } \\
\text { outpatient services) }\end{array}$ & 127 & & & 146 & & & 140 & & & 150 & & \\
\hline $\begin{array}{l}\text { Orthopaedic clinic } \\
\text { (for shoulder) }\end{array}$ & 123 & 96.85 & $0.03(0.22)$ & 141 & 96.58 & $0.03(0.17)$ & 135 & 96.43 & $0.03(0.21)$ & 148 & 98.67 & 0 \\
\hline $\begin{array}{l}\text { Physiotherapy department } \\
\text { (not as part of the } \\
\text { GRASP trial) }\end{array}$ & 122 & 96.06 & $0.02(0.13)$ & 138 & 94.52 & $0.04(0.43)$ & 135 & 96.43 & $0.07(0.49)$ & 150 & 100 & $0.03(0.28)$ \\
\hline Radiology: X-ray & 121 & 95.28 & $0.01(0.11)$ & 137 & 93.84 & $0.02(0.13)$ & 132 & 94.29 & $0.01(0.11)$ & 148 & 98.67 & 0 \\
\hline Radiology: ultrasound & 121 & 95.28 & 0 & 137 & 93.84 & 0 & 132 & 94.29 & 0 & 148 & 98.67 & 0 \\
\hline Radiology: MRI & 121 & 95.28 & 0 & 137 & 93.84 & 0 & 132 & 94.29 & 0 & 148 & 98.67 & 0 \\
\hline Accident and emergency & 121 & 95.28 & $0.01(0.09)$ & 137 & 93.84 & 0 & 132 & 94.29 & 0 & 148 & 98.67 & 0 \\
\hline Other outpatient services & 121 & 95.28 & $0.01(0.09)$ & 137 & 93.84 & 0 & 132 & 94.29 & 0 & 148 & 98.67 & 0 \\
\hline $\begin{array}{l}\text { Secondary care (NHS inpatient } \\
\text { services) }\end{array}$ & 127 & & & 147 & & & 140 & & & 150 & & \\
\hline Inpatient care & 127 & 100 & 0 & 147 & 100 & $0.03(0.15)$ & 140 & 100 & 0 & 150 & 100 & 0 \\
\hline
\end{tabular}


TABLE 46 Mean health resource utilisation: 8 weeks to 6 months (available-case analysis, excluding patients with missing data on that resource) (continued)

\begin{tabular}{|c|c|c|c|c|c|c|c|c|c|c|c|c|}
\hline \multirow[b]{2}{*}{ Type of care } & \multicolumn{3}{|c|}{ Best-practice advice $(N=144)$} & \multicolumn{3}{|c|}{$\begin{array}{l}\text { Injection plus best-practice } \\
\text { advice ( }=158)\end{array}$} & \multicolumn{3}{|c|}{ Progressive exercise $(N=151)$} & \multicolumn{3}{|c|}{$\begin{array}{l}\text { Injection plus progressive } \\
\text { exercise }(N=162)\end{array}$} \\
\hline & $\boldsymbol{n}^{\mathrm{a}}$ & $\begin{array}{l}\text { Completion } \\
\text { rate }^{\mathrm{b}}(\%)\end{array}$ & 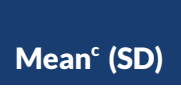 & $n^{a}$ & $\begin{array}{l}\text { Completion } \\
\text { rate }^{\mathrm{b}}(\%)\end{array}$ & Mean' (SD) & $n^{\mathrm{a}}$ & $\begin{array}{l}\text { Completion } \\
\text { rate }^{\mathrm{b}}(\%)\end{array}$ & Mean' (SD) & $n^{a}$ & $\begin{array}{l}\text { Completion } \\
\text { rate }^{\mathrm{b}}(\%)\end{array}$ & $\operatorname{Mean}^{c}$ (SD) \\
\hline Private care & 128 & & & 147 & & & 140 & & & 150 & & \\
\hline $\begin{array}{l}\text { Orthopaedic clinic } \\
\text { (for shoulder) }\end{array}$ & 121 & 94.53 & $0.02(0.18)$ & 141 & 95.92 & 0 & 132 & 94.29 & 0 & 147 & 98 & $0.01(0.08)$ \\
\hline Physiotherapy department & 124 & 96.88 & $0.08(0.45)$ & 143 & 97.28 & $0.12(1.26)$ & 134 & 95.71 & $0.01(0.12)$ & 150 & 100 & $0.07(0.53)$ \\
\hline Radiology: X-ray & 120 & 93.75 & 0 & 141 & 95.92 & 0 & 132 & 94.29 & 0 & 147 & 98 & 0 \\
\hline Radiology: ultrasound & 120 & 93.75 & 0 & 141 & 95.92 & 0 & 132 & 94.29 & 0 & 146 & 97.33 & 0 \\
\hline Radiology: MRI & 120 & 93.75 & 0 & 141 & 95.92 & 0 & 132 & 94.29 & 0 & 146 & 97.33 & 0 \\
\hline Chiropractor & 122 & 95.31 & $0.03(0.29)$ & 142 & 96.6 & $0.04(0.50)$ & 135 & 96.43 & $0.15(1.19)$ & 147 & 98 & $0.01(0.08)$ \\
\hline Complementary therapist & 122 & 95.31 & $0.05(0.4)$ & 142 & 96.6 & $0.01(0.17)$ & 133 & 95 & $0.02(0.26)$ & 146 & 97.33 & 0 \\
\hline Injection utilisation & 130 & & & 147 & & & 140 & & & 149 & & \\
\hline Injections & 130 & 100 & $0.07(0.25)$ & 147 & 100 & $0.04(0.19)$ & 140 & 100 & $0.08(0.32)$ & 149 & 100 & $0.06(0.30)$ \\
\hline Non-medical expenses & 129 & & & 147 & & & 140 & & & 150 & & \\
\hline
\end{tabular}


TABLE 47 Mean health resource utilisation: 6-12 months (available-case analysis, excluding patients with missing data on that resource)

\begin{tabular}{|c|c|c|c|c|c|c|c|c|c|c|c|c|}
\hline \multirow[b]{2}{*}{ Type of care } & \multicolumn{3}{|c|}{ Best-practice advice $(N=143)$} & \multicolumn{3}{|c|}{$\begin{array}{l}\text { Injection plus best-practice } \\
\text { advice }(N=161)\end{array}$} & \multicolumn{3}{|c|}{ Progressive exercise $(N=153)$} & \multicolumn{3}{|c|}{$\begin{array}{l}\text { Injection plus progressive } \\
\text { exercise }(N=161)\end{array}$} \\
\hline & $n^{a}$ & $\begin{array}{l}\text { Completion } \\
\text { rate }^{\mathrm{b}}(\%)\end{array}$ & Meanc (SD) & $n^{\mathrm{a}}$ & $\begin{array}{l}\text { Completion } \\
\text { rate }^{\mathrm{b}}(\%)\end{array}$ & $\operatorname{Mean}^{c}$ (SD) & $n^{a}$ & $\begin{array}{l}\text { Completion } \\
\text { rate }^{\mathrm{b}}(\%)\end{array}$ & $\operatorname{Mean}^{c}$ (SD) & $n^{\mathrm{a}}$ & $\begin{array}{l}\text { Completion } \\
\text { rate }^{\mathrm{b}}(\%)\end{array}$ & 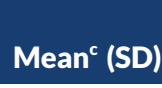 \\
\hline $\begin{array}{l}\text { Primary care (NHS community- } \\
\text { based services) }\end{array}$ & 133 & & & 150 & & & 140 & & & 152 & & \\
\hline GP visit & 129 & 96.99 & $0.19(0.69)$ & 145 & 96.67 & $0.21(0.63)$ & 138 & 98.57 & $0.21(0.84)$ & 147 & 96.71 & $0.26(0.83)$ \\
\hline GP telephone contact & 118 & 88.72 & $0.03(0.21)$ & 130 & 86.67 & $0.02(0.19)$ & 126 & 90 & 0 & 133 & 87.5 & $0.13(0.92)$ \\
\hline GP home visit & 116 & 87.22 & 0 & 128 & 85.33 & 0 & 126 & 90 & 0 & 129 & 84.87 & $0.01(0.09)$ \\
\hline Practice nurse & 116 & 87.22 & 0 & 128 & 85.33 & 0 & 126 & 90 & 0 & 128 & 84.21 & 0 \\
\hline $\begin{array}{l}\text { Physiotherapy (not as part } \\
\text { of the GRASP trial) }\end{array}$ & 120 & 90.23 & $0.1(0.67)$ & 133 & 88.67 & $0.11(0.71)$ & 130 & 92.86 & $0.09(0.60)$ & 135 & 88.82 & $0.16(0.81)$ \\
\hline $\begin{array}{l}\text { Other NHS community } \\
\text { services }\end{array}$ & 118 & 88.72 & $0.03(0.21)$ & 131 & 87.33 & $0.02(0.15)$ & 129 & 92.14 & $0.02(0.15)$ & 129 & 84.87 & $0.01(0.09)$ \\
\hline $\begin{array}{l}\text { Secondary care (NHS } \\
\text { outpatient services) }\end{array}$ & 133 & & & 150 & & & 141 & & & 152 & & \\
\hline $\begin{array}{l}\text { Orthopaedic clinic } \\
\text { (for shoulder) }\end{array}$ & 128 & 96.24 & $0.08(0.45)$ & 140 & 93.33 & $0.07(0.39)$ & 135 & 95.74 & $0.04(0.32)$ & 147 & 96.71 & $0.12(0.47)$ \\
\hline $\begin{array}{l}\text { Physiotherapy department } \\
\text { (not as part of the } \\
\text { GRASP trial) }\end{array}$ & 126 & 94.74 & $0.06(0.49)$ & 142 & 94.67 & $0.13(0.70)$ & 133 & 94.33 & $0.02(0.17)$ & 140 & 92.11 & $0.04(0.31)$ \\
\hline Radiology: X-ray & 126 & 94.74 & $0.01(0.11)$ & 142 & 94.67 & $0.02(0.17)$ & 133 & 94.33 & $0.02(0.19)$ & 140 & 92.11 & $0.01(0.11)$ \\
\hline Radiology: ultrasound & 126 & 94.74 & $0.01(0.07)$ & 142 & 94.67 & 0 & 133 & 94.33 & 0 & 140 & 92.11 & $0.01(0.07)$ \\
\hline Radiology: MRI & 126 & 94.74 & $0.01(0.07)$ & 142 & 94.67 & $0.01(0.08)$ & 133 & 94.33 & $0.01(0.08)$ & 140 & 92.11 & $0.02(0.13)$ \\
\hline Accident and emergency & 124 & 93.23 & $0.01(0.08)$ & 137 & 91.33 & $0.01(0.12)$ & 132 & 93.62 & 0 & 137 & 90.13 & 0 \\
\hline Other outpatient services & 124 & 93.23 & $0.02(0.18)$ & 136 & 90.67 & $0.01(0.17)$ & 132 & 93.62 & 0 & 138 & 90.79 & $0.01(0.17)$ \\
\hline
\end{tabular}


TABLE 47 Mean health resource utilisation: 6-12 months (available-case analysis, excluding patients with missing data on that resource) (continued)

\begin{tabular}{|c|c|c|c|c|c|c|c|c|c|c|c|c|}
\hline \multirow[b]{2}{*}{ Type of care } & \multicolumn{3}{|c|}{ Best-practice advice $(N=143)$} & \multicolumn{3}{|c|}{$\begin{array}{l}\text { Injection plus best-practice } \\
\text { advice }(N=161)\end{array}$} & \multicolumn{3}{|c|}{ Progressive exercise $(N=153)$} & \multicolumn{3}{|c|}{$\begin{array}{l}\text { Injection plus progressive } \\
\text { exercise }(N=161)\end{array}$} \\
\hline & $n^{\mathrm{a}}$ & $\begin{array}{l}\text { Completion } \\
\text { rate }^{\mathrm{b}}(\%)\end{array}$ & 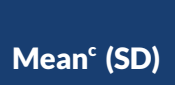 & $n^{\mathrm{a}}$ & $\begin{array}{l}\text { Completion } \\
\text { rate }^{\mathrm{b}}(\%)\end{array}$ & Mean $^{c}$ (SD) & $n^{\mathrm{a}}$ & $\begin{array}{l}\text { Completion } \\
\text { rate }^{\mathrm{b}}(\%)\end{array}$ & Mean $^{c}$ (SD) & $n^{\mathrm{a}}$ & $\begin{array}{l}\text { Completion } \\
\text { rate }^{\mathrm{b}}(\%)\end{array}$ & 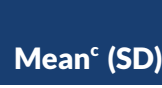 \\
\hline $\begin{array}{l}\text { Secondary care (NHS inpatient } \\
\text { services) }\end{array}$ & 132 & & & 150 & & & 140 & & & 152 & & \\
\hline Inpatient care & 133 & & & 150 & & & 141 & & & 152 & & \\
\hline Private care & 126 & 94.74 & $0.02(0.18)$ & 147 & 98 & 0 & 134 & 95.04 & $0.01(0.17)$ & 145 & 95.39 & $0.02(0.19)$ \\
\hline $\begin{array}{l}\text { Orthopaedic clinic } \\
\text { (for shoulder) }\end{array}$ & 127 & 95.49 & $0.05(0.37)$ & 148 & 98.67 & $0.01(0.08)$ & 136 & 96.45 & $0.05(0.37)$ & 148 & 97.37 & $0.11(0.66)$ \\
\hline Physiotherapy department & 126 & 94.74 & 0 & 147 & 98 & 0 & 135 & 95.74 & 0 & 148 & 97.37 & $0.01(0.07)$ \\
\hline Radiology: X-ray & 126 & 94.74 & 0 & 147 & 98 & 0 & 135 & 95.74 & 0 & 148 & 97.37 & 0 \\
\hline Radiology: ultrasound & 126 & 94.74 & 0 & 147 & 98 & 0 & 135 & 95.74 & 0 & 148 & 97.37 & $0.01(0.07)$ \\
\hline Radiology: MRI & 126 & 94.74 & $0.01(0.09)$ & 147 & 98 & 0 & 135 & 95.74 & $0.05(0.42)$ & 145 & 95.39 & $0.03(0.26)$ \\
\hline Chiropractor & 126 & 94.74 & $0.07(0.80)$ & 148 & 98.67 & $0.10(1.23)$ & 134 & 95.04 & $0.02(0.27)$ & 143 & 94.08 & 0 \\
\hline Complementary therapist & 133 & & & 150 & & & 141 & & & 150 & & \\
\hline Injection utilisation & 133 & & $0.11(0.35)$ & 150 & & $0.07(0.29)$ & 141 & & $0.09(0.35)$ & 150 & & $0.1(0.36)$ \\
\hline Injections & 133 & & & 150 & & & 141 & & & 152 & & \\
\hline Non-medical expenses & 133 & & & 150 & & & 140 & & & 152 & & \\
\hline
\end{tabular}


TABLE 48 Mean NHS costs (SD) by treatment group and follow-up time point (2019 prices): baseline to 8 weeks (available cases)

\begin{tabular}{|c|c|c|c|c|c|c|c|c|}
\hline \multirow[b]{2}{*}{ Type of care } & \multicolumn{2}{|c|}{$\begin{array}{l}\text { Best-practice } \\
\text { advice }(N=150) \\
\end{array}$} & \multicolumn{2}{|c|}{$\begin{array}{l}\text { Injection plus } \\
\text { best-practice } \\
\text { advice }(N=165) \\
\end{array}$} & \multicolumn{2}{|c|}{$\begin{array}{l}\text { Progressive } \\
\text { exercise } \\
(N=157) \\
\end{array}$} & \multicolumn{2}{|c|}{$\begin{array}{l}\text { Injection plus } \\
\text { progressive } \\
\text { exercise }(N=169)\end{array}$} \\
\hline & $\begin{array}{l}\text { Mean } \\
\text { cost (£) }\end{array}$ & SD & $\begin{array}{l}\text { Mean } \\
\text { cost }(£)\end{array}$ & SD & $\begin{array}{l}\text { Mean } \\
\text { cost }(£)\end{array}$ & SD & $\begin{array}{l}\text { Mean } \\
\text { cost (£) }\end{array}$ & SD \\
\hline
\end{tabular}

Primary care (NHS community-based services)

$\begin{array}{lrrrrrrrr}\text { GP visit } & 5.86 & 18.54 & 2.36 & 11.38 & 5.11 & 7.54 & 3.57 & 13.87 \\ \text { GP telephone contact } & 1.18 & 6.68 & 0.19 & 2.31 & 0.44 & 4.94 & 1.43 & 13.69 \\ \text { Practice nurse } & 0.74 & 7.87 & 0.00 & 0.00 & 1.68 & 18.78 & 0.55 & 4.77 \\ \begin{array}{l}\text { Physiotherapy (not as part } \\ \text { of the GRASP trial) }\end{array} & 1.93 & 20.69 & 0.26 & 3.08 & 4.46 & 18.78 & 0.72 & 5.11\end{array}$

Secondary care (NHS outpatient services)

$\begin{array}{lrrrrrrrr}\text { Orthopaedic clinic (for shoulder) } & 0.97 & 10.78 & 1.66 & 19.93 & 0.00 & 0.00 & 0.00 & 0.00 \\ \begin{array}{l}\text { Physiotherapy department } \\ \text { (not as part of the }\end{array} & 0.00 & 0.00 & 1.33 & 16.11 & 1.12 & 10.42 & 0.96 & 12.23 \\ \begin{array}{l}\text { GRASP trial) } \\ \text { Radiology: X-ray }\end{array} & & & & & & & & \\ \text { Radiology: ultrasound } & 0.49 & 3.91 & 0.63 & 4.39 & 2.36 & 10.42 & 0.00 & 0.00 \\ \text { Accident and emergency } & 0.31 & 3.50 & 0.79 & 5.53 & 0.00 & 0.00 & 0.48 & 4.32 \\ \text { tal } & 1.69 & 13.35 & 2.16 & 15.03 & 0.00 & 0.00 & 0.00 & 0.00 \\ & 9.31 & 35.65 & 7.75 & 39.2 & 11.82 & 35.95 & 4.85 & 23.37\end{array}$

TABLE 49 Mean NHS costs (SD) by treatment group and follow-up time point (2019 prices): 8 weeks to 6 months (available cases)

\begin{tabular}{|c|c|c|c|c|}
\hline & $\begin{array}{l}\text { Best-practice } \\
\text { advice }(N=150)\end{array}$ & $\begin{array}{l}\text { Injection plus } \\
\text { best-practice } \\
\text { advice }(N=165)\end{array}$ & $\begin{array}{l}\text { Progressive } \\
\text { exercise } \\
(N=157)\end{array}$ & $\begin{array}{l}\text { Injection plus } \\
\text { progressive } \\
\text { exercise }(N=169)\end{array}$ \\
\hline Type of care & $\begin{array}{l}\text { Mean } \\
\text { cost (£) }\end{array}$ & $\begin{array}{l}\text { Mean } \\
\text { cost }(£)\end{array}$ & $\begin{array}{l}\text { Mean } \\
\text { cost }(£)\end{array}$ & $\begin{array}{l}\text { Mean } \\
\text { cost (£) }\end{array}$ \\
\hline
\end{tabular}

Primary care (NHS community-based services)

$\begin{array}{lrrrrrrrr}\text { GP visit } & 6.05 & 23.03 & 3.59 & 13.94 & 6.39 & 22.07 & 5.52 & 17.67 \\ \text { GP telephone contact } & 2.12 & 12.11 & 0.21 & 2.39 & 0.45 & 4.98 & 0.20 & 2.38 \\ \text { Practice nurse } & 0.37 & 3.95 & 0.00 & 0.00 & 0.00 & 0.00 & 0.00 & 0.00 \\ \begin{array}{l}\text { Physiotherapy (not as part of } \\ \text { the GRASP trial) }\end{array} & 6.92 & 41.01 & 3.46 & 21.28 & 2.92 & 18.98 & 0.81 & 7.04\end{array}$

Secondary care (NHS outpatient services)

\begin{tabular}{|c|c|c|c|c|c|c|c|c|}
\hline Orthopaedic clinic (for shoulder) & 3.9 & 26.32 & 3.4 & 19.99 & 3.55 & 25.14 & 0.00 & 0.00 \\
\hline $\begin{array}{l}\text { Physiotherapy department } \\
\text { (not as part of the GRASP trial) }\end{array}$ & 0.64 & 4.95 & 1.41 & 16.52 & 2.59 & 19.08 & 1.03 & 9.99 \\
\hline Radiology: X-ray & 0.36 & 3.31 & 0.52 & 4.00 & 0.36 & 3.31 & 0.00 & 0.00 \\
\hline Accident and emergency & 0.88 & 9.64 & 0.00 & 0.00 & 0.00 & 0.00 & 0.00 & 0.00 \\
\hline \multicolumn{9}{|c|}{ condary care (NHS inpatient services) } \\
\hline Inpatient care & 0.00 & 0.00 & 24.64 & 298.82 & 0.00 & 0.00 & 0.00 & 0.00 \\
\hline tal & 14.78 & 59.69 & 30.37 & 293.54 & 12.51 & 44.02 & 6.13 & 20.59 \\
\hline
\end{tabular}


TABLE 50 Mean NHS costs (SD) by treatment group and follow-up time point (2019 prices): 6-12 months (available cases)

\begin{tabular}{|c|c|c|c|c|c|c|c|c|}
\hline \multirow[b]{2}{*}{ Type of care } & \multicolumn{2}{|c|}{$\begin{array}{l}\text { Best-practice } \\
\text { advice }(\mathrm{N}=150)\end{array}$} & \multicolumn{2}{|c|}{$\begin{array}{l}\text { Injection plus } \\
\text { best-practice } \\
\text { advice }(N=165)\end{array}$} & \multicolumn{2}{|c|}{$\begin{array}{l}\text { Progressive } \\
\text { exercise } \\
(N=157)\end{array}$} & \multicolumn{2}{|c|}{$\begin{array}{l}\text { Injection plus } \\
\text { progressive } \\
\text { exercise }(N=169)\end{array}$} \\
\hline & $\begin{array}{l}\text { Mean } \\
\text { cost (£) }\end{array}$ & SD & $\begin{array}{l}\text { Mean } \\
\text { cost (f) }\end{array}$ & SD & $\begin{array}{l}\text { Mean } \\
\text { cost }(\boldsymbol{f})\end{array}$ & SD & $\begin{array}{l}\text { Mean } \\
\text { cost (€) }\end{array}$ & SD \\
\hline
\end{tabular}

Primary care (NHS community-based services)

$\begin{array}{lrrrrrrrr}\text { GP visit } & 7.60 & 27.33 & 8.11 & 24.85 & 8.24 & 32.99 & 10.40 & 32.57 \\ \text { GP telephone contact } & 0.70 & 5.67 & 0.64 & 5.39 & 0.00 & 0.00 & 3.53 & 25.31 \\ \text { GP home visit } & 0.00 & 0.00 & 0.00 & 0.00 & 0.00 & 0.00 & 0.04 & 0.49 \\ \begin{array}{l}\text { Physiotherapy (not as part of } \\ \text { the GRASP trial) }\end{array} & 3.68 & 24.53 & 4.15 & 25.91 & 3.40 & 22.21 & 6.00 & 29.92\end{array}$

Secondary care (NHS outpatient services)

\begin{tabular}{|c|c|c|c|c|c|c|c|c|}
\hline Orthopaedic clinic (for shoulder) & 9.37 & 53.47 & 8.57 & 46.95 & 5.33 & 38.41 & 13.87 & 57.08 \\
\hline $\begin{array}{l}\text { Physiotherapy department } \\
\text { (not as part of the } \\
\text { GRASP trial) }\end{array}$ & 2.46 & 18.84 & 4.91 & 27.28 & 0.58 & 6.73 & 1.66 & 12.20 \\
\hline Radiology: X-ray & 0.36 & 3.33 & 0.53 & 5.21 & 0.72 & 5.75 & 0.35 & 3.27 \\
\hline Radiology: ultrasound & 0.23 & 2.98 & 0.00 & 0.00 & 0.00 & 0.00 & 0.22 & 2.92 \\
\hline Radiology: MRI & 0.63 & 8.26 & 0.62 & 8.16 & 0.63 & 8.25 & 1.80 & 13.86 \\
\hline Accident and emergency & 0.85 & 9.52 & 1.55 & 12.76 & 0.00 & 0.00 & 0.00 & 0.00 \\
\hline \multicolumn{9}{|c|}{ econdary care (NHS inpatient services) } \\
\hline Inpatient care & 22.83 & 261.35 & 0.00 & 0.00 & 0.00 & 0.00 & 23.99 & 294.84 \\
\hline otal & 36.33 & 293.35 & 23.16 & 84.3 & 14.99 & 56.62 & 50.19 & 309.46 \\
\hline
\end{tabular}

TABLE 51 Mean prescribed drug cost

\begin{tabular}{|c|c|c|c|c|c|c|c|c|}
\hline \multirow[b]{2}{*}{ Time point } & \multicolumn{2}{|c|}{ Best-practice advice } & \multicolumn{2}{|c|}{$\begin{array}{l}\text { Injection plus } \\
\text { best-practice advice }\end{array}$} & \multicolumn{2}{|c|}{$\begin{array}{l}\text { Progressive } \\
\text { exercise }\end{array}$} & \multicolumn{2}{|c|}{$\begin{array}{l}\text { Injection plus } \\
\text { progressive exercise }\end{array}$} \\
\hline & $n^{a}$ & $\begin{array}{l}\text { Mean cost (E) } \\
\text { (SD) }\end{array}$ & $n^{a}$ & $\begin{array}{l}\text { Mean cost ( } \mathrm{E}) \\
\text { (SD) }\end{array}$ & $n^{a}$ & $\begin{array}{l}\text { Mean cost (E) } \\
\text { (SD) }\end{array}$ & $n^{a}$ & $\begin{array}{l}\text { Mean cost (£) } \\
\text { (SD) }\end{array}$ \\
\hline Baseline to 8 weeks & 150 & $6.64(57.73)$ & 165 & $0.74(3.32)$ & 157 & $0.71(2.70)$ & 169 & $5.11(54.02)$ \\
\hline 8 weeks to 6 months & 144 & $1.94(8.09)$ & 158 & $1.74(6.93)$ & 151 & $0.75(3.17)$ & 162 & 3.28 (11.69) \\
\hline 6-12 months & 143 & $1.65(5.62)$ & 161 & 2.57 (11.27) & 153 & $1.17(6.55)$ & 161 & $7.87(34.81)$ \\
\hline
\end{tabular}


TABLE 52 Utilisation of steroid injections outside the GRASP trial

\begin{tabular}{|c|c|c|c|c|c|c|c|c|c|c|c|c|}
\hline \multirow[b]{2}{*}{ Time point } & \multicolumn{3}{|c|}{ Best-practice advice } & \multicolumn{3}{|c|}{ Injection plus best-practice advice } & \multicolumn{3}{|c|}{ Progressive exercise } & \multicolumn{3}{|c|}{ Injection plus progressive exercise } \\
\hline & $n(\%)$ & $\begin{array}{l}\text { Privately } \\
\text { paid, \% }\end{array}$ & Mean (SD) & $n(\%)$ & $\begin{array}{l}\text { Privately } \\
\text { paid, \% }\end{array}$ & Mean (SD) & $n(\%)$ & $\begin{array}{l}\text { Privately } \\
\text { paid, \% }\end{array}$ & Mean (SD) & $n(\%)$ & $\begin{array}{l}\text { Privately } \\
\text { paid, \% }\end{array}$ & Mean (SD) \\
\hline $\begin{array}{l}\text { Baseline to } \\
8 \text { weeks }\end{array}$ & $1(0.77)$ & 0 & $0.81(9.17)$ & $7(4.63)$ & 0 & $2.78(13.22)$ & $3(2.04)$ & 33.33 & $1.07(7.42)$ & $10(6.13)$ & 0 & $3.21(12.59)$ \\
\hline $\begin{array}{l}8 \text { weeks to } \\
6 \text { months }\end{array}$ & $9(6.92)$ & 0.77 & $3.62(13.33)$ & $6(4.08)$ & 0 & $2.14(10.38)$ & $9(6.43)$ & 0.71 & $4.11(16.68)$ & $6(4.03)$ & 0.67 & $2.81(15.84)$ \\
\hline 6-12 months & $12(9.02)$ & 0.75 & $5.51(18.51)$ & $8(5.33)$ & 0 & $3.49(15.65)$ & $9(6.39)$ & 0 & $4.45(18.22)$ & $12(8)$ & 0.67 & $5.23(18.93)$ \\
\hline
\end{tabular}


TABLE 53 Utility and QALY estimates: EQ-5D visual analogue scale scores (available cases with no adjustment for baseline utility)

\begin{tabular}{|c|c|c|c|c|c|c|c|c|}
\hline \multirow[b]{2}{*}{ Time point } & \multicolumn{2}{|c|}{ Best-practice advice } & \multicolumn{2}{|c|}{$\begin{array}{l}\text { Injection plus } \\
\text { best-practice advice }\end{array}$} & \multicolumn{2}{|c|}{ Progressive exercise } & \multicolumn{2}{|c|}{$\begin{array}{l}\text { Injection plus } \\
\text { progressive exercise }\end{array}$} \\
\hline & $\boldsymbol{n}^{\mathrm{a}}$ & Mean (SE) & $n^{\mathrm{a}}$ & Mean (SE) & $n^{\mathrm{a}}$ & Mean (SE) & $n^{a}$ & Mean (SE) \\
\hline Baseline & 174 & $74.68(1.42)$ & 178 & $77.34(1.16)$ & 172 & 77.25 (1.17) & 181 & $75.55(1.31)$ \\
\hline 8 weeks & 132 & 77.04 (1.24) & 151 & $79.95(1.11)$ & 147 & 78.89 (1.17) & 165 & 76.78 (1.38) \\
\hline 6 months & 134 & 78.49 (1.44) & 151 & $80.68(1.20)$ & 143 & $81.36(1.29)$ & 154 & $78.33(1.50)$ \\
\hline 12 months & 138 & $81.30(1.26)$ & 158 & $79.32(1.23)$ & 143 & $82.17(1.33)$ & 154 & $80.21(1.33)$ \\
\hline $\begin{array}{l}\text { Change from } \\
\text { baseline to } \\
12 \text { months }\end{array}$ & 107 & $4.65(18.27)$ & 133 & $1.22(12.53)$ & 123 & $1.70(11.47)$ & 139 & $1.26(15.53)$ \\
\hline QALYs & 107 & $0.73(0.17)$ & 132 & $0.75(0.12)$ & 124 & $0.76(0.12)$ & 137 & $0.72(0.18)$ \\
\hline
\end{tabular}

a Relates to the number of participants for whom follow-up data were available.

TABLE 54 Regression analysis without interaction term, including imputation of missing values and adjustment for baseline utility, sex and age

\begin{tabular}{|c|c|c|c|}
\hline & Total costs (£), mean (SE) & QALYs, mean (SE) & NMB (£), ${ }^{a}$ mean (SE) \\
\hline Injection & $256(17)$ & 0.749 (0.009) & $14,729(185)$ \\
\hline No injection & $221(35)$ & $0.747(0.010)$ & $14,715(221)$ \\
\hline Injection main effect & $35(31)(p=0.239)$ & $0.003(0.011)(p=0.818)$ & $15(233)(p=0.947)$ \\
\hline Progressive exercise & $277(18)$ & $0.748(0.010)$ & $14,685(201)$ \\
\hline No progressive exercise & $199(34)$ & $0.748(0.010)$ & $14,758(201)$ \\
\hline Progressive exercise main effect & $78(30)(p=0.012)$ & $0.00(0.011)(p=0.984)$ & $-73(231)(p=0.753)$ \\
\hline \multicolumn{4}{|c|}{$\begin{array}{l}\text { a NMB calculated at a ceiling ratio of } £ 20,000 \text { per QALY. } \\
\text { Notes } \\
\text { Values represent the mean (SE) for each group for males of age } 55.46 \text { years and a baseline utility of } 0.653 \text {. As there } \\
\text { was assumed to be no interaction between baseline variables and treatments, the simple effects for each treatment } \\
\text { and the interaction between treatments are assumed to be the same for all participant subgroups, although the } \\
\text { absolute costs and absolute QALYs may vary, depending on participants' sex, age and baseline utility. }\end{array}$} \\
\hline
\end{tabular}

TABLE 55 Regression analysis with an interaction term, including both NHS and broader societal perspective

\begin{tabular}{|c|c|c|c|}
\hline & $\begin{array}{l}\text { Total societal costs }(\boldsymbol{E}) \text {, } \\
\text { mean (SE) }\end{array}$ & QALYs, mean (SE) & NMB (E), ${ }^{a}$ mean (SE) \\
\hline Best-practice advice & 396 (159) & $0.737(0.014)$ & $14,340(344)$ \\
\hline Injection plus best-practice advice & $362(118)$ & $0.757(0.011)$ & $14,783(266)$ \\
\hline Progressive exercise & $336(108)$ & $0.756(0.012)$ & $14,778(285)$ \\
\hline Injection plus progressive exercise & $710(247)$ & $0.741(0.012)$ & $14,118(366)$ \\
\hline Injection simple effect & $-34(137)(p=0.813)$ & $0.020(0.015)(p=0.183)$ & $443(359)(p=0.218)$ \\
\hline Exercise simple effect & $-60(113)(p=0.604)$ & $0.019(0.016)(p=0.229)$ & $438(352)(p=0.215)$ \\
\hline Interaction: exercise by injection & $408(343)(p=0.194)$ & $-0.035(0.022)(p=0.106)$ & $-1103(583)(p=0.049)$ \\
\hline \multicolumn{4}{|c|}{$\begin{array}{l}\text { a NMB calculated at a ceiling ratio of } £ 20,000 \text { per QALY. } \\
\text { Notes } \\
\text { Values represent the mean (SE) for each group for males of age } 55.46 \text { years and a baseline utility of } 0.653 \text {. As there } \\
\text { was assumed to be no interaction between baseline variables and treatments, the simple effects for each treatment } \\
\text { and the interaction between treatments are assumed to be the same for all patient subgroups, although the absolute } \\
\text { costs and absolute QALYs may vary, depending on patients' sex, age and baseline utility. }\end{array}$} \\
\hline
\end{tabular}


TABLE 56 Results of the worst-case scenario for training costs: regression analysis with an interaction term, including imputation of missing values and adjustment for baseline utility, sex and age

\begin{tabular}{llll} 
& $\begin{array}{l}\text { Total costs }(\boldsymbol{\epsilon}), \\
\text { mean }(\mathbf{S E})\end{array}$ & QALYs, mean (SE) & NMB (£), ${ }^{\mathrm{a}}$ mean (SE) \\
\hline Best-practice advice & $295(69)$ & $0.738(0.018)$ & $14,469(384)$ \\
Injection plus best-practice advice & $303(28)$ & $0.760(0.016)$ & $14,905(327)$ \\
Progressive exercise & $388(29)$ & $0.757(0.019)$ & $14,754(383)$ \\
Injection plus progressive exercise & $449(45)$ & $0.745(0.016)$ & $14,441(327)$ \\
Injection simple effect & $8(58)(p=0.737)$ & $0.022(0.022)(p=0.301)$ & $436(449)(p=0.326)$ \\
Exercise simple effect & $92(55)(p=0.138)$ & $0.019(0.022)(p=0.368)$ & $285(460)(p=0.504)$ \\
Interaction: exercise by injection & $53(72)(p=0.495)$ & $-0.035(0.033)(p=0.285)$ & $-749(678)(p=0.259)$ \\
\hline
\end{tabular}

a NMB calculated at a ceiling ratio of $£ 20,000$ per QALY.

Notes

Values represent the mean (SE) for each group for males of age 55.46 years and a baseline utility of 0.653 . As there was assumed to be no interaction between baseline variables and treatments, the simple effects for each treatment and the interaction between treatments are assumed to be the same for all patient subgroups, although the absolute costs and absolute QALYs may vary, depending on patients' sex, age and baseline utility.

TABLE 57 Results of the best-case scenario: regression analysis with an interaction term, including imputation of missing values and adjustment for baseline utility, sex and age

\begin{tabular}{llll} 
& $\begin{array}{l}\text { Total costs }(\boldsymbol{\epsilon}), \\
\text { mean }(\mathbf{S E})\end{array}$ & QALYs, mean (SE) & NMB (£), ${ }^{\mathrm{a}}$ mean (SE) \\
\hline Best-practice advice & $149(54)$ & $0.737(0.013)$ & $14,584(291)$ \\
Injection plus best-practice advice & $160(20)$ & $0.757(0.011)$ & $14,986(229)$ \\
Progressive exercise & $195(22)$ & $0.756(0.013)$ & $14,920(257)$ \\
Injection plus progressive exercise & $255(30)$ & $0.741(0.012)$ & $14,572(262)$ \\
Injection simple effect & $11(44)(p=0.722)$ & $0.021(0.015)(p=176)$ & $403(320)(p=0.206)$ \\
Exercise simple effect & $46(44)(p=0.299)$ & $0.019(0.016)(p=0.217)$ & $337(325)(p=0.297)$ \\
Interaction: exercise by injection & $49(57)(p=0.399)$ & $-0.035(0.022)(p=0.108)$ & $-751(457)(p=0.100)$ \\
\hline
\end{tabular}

a NMB calculated at a ceiling ratio of $£ 20,000$ per QALY.

Notes

Values represent the mean (SE) for each group for males of age 55.46 years and a baseline utility of 0.653 . As there was assumed to be no interaction between baseline variables and treatments, the simple effects for each treatment and the interaction between treatments are assumed to be the same for all patient subgroups, although the absolute costs and absolute QALYs may vary, depending on patients' sex, age and baseline utility. 



\section{Appendix 6 Chapter 6 appendix}


TABLE 58 Summary of trials comparing the effects of supervised vs. unsupervised exercise interventions

\begin{tabular}{|c|c|c|c|c|c|c|c|c|}
\hline Study & Design/follow-up & Participants & Intervention & Comparator & Outcomes & Number analysed & Results & Conclusion \\
\hline $\begin{array}{l}\text { Krischak et al. } \\
(2013)^{110}\end{array}$ & $\begin{array}{l}\text { Pilot RCT } \\
\text { Two arms } \\
\text { Follow-up: } \\
2 \text { months }\end{array}$ & $\begin{array}{l}\text { Forty-three patients with } \\
\text { unilateral, symptomatic, } \\
\text { atraumatic full-thickness } \\
\text { rotator cuff tears } \\
\text { verified by MRI (usual } \\
\text { physiotherapy, } n=23 ; \\
\text { home exercise, } n=20 \text { ); } \\
14 \text { females: } 24 \text { males; } \\
\text { mean age } 55.3 \text { (11.8 SD) } \\
\text { years; analysed } \\
\text { participants only }\end{array}$ & $\begin{array}{l}\text { Usual physiotherapy } \\
\text { Physiotherapy with } \\
\text { supervised exercises } \\
\text { (duration } 8 \text { weeks, } \\
\text { frequency three times } \\
\text { per week) } \\
\text { Content of the } \\
\text { rehabilitation } \\
\text { programme determined } \\
\text { exclusively by the } \\
\text { treating therapist } \\
\text { (supervised) }\end{array}$ & $\begin{array}{l}\text { Home (unsupervised) } \\
\text { exercise } \\
\text { Received an exercise } \\
\text { guide booklet with } \\
\text { detailed instructions } \\
\text { and demonstrations. } \\
\text { Contents of booklet } \\
\text { discussed and patients } \\
\text { taught how to perform } \\
\text { exercises correctly } \\
\\
\text { Exercises were aimed at } \\
\text { restoring neuromuscular } \\
\text { control at the shoulder, } \\
\text { as well as strength and } \\
\text { ROM. Contents of booklet } \\
\text { instructed patients on } \\
\text { the type of exercises, } \\
\text { repetitions, intensity, } \\
\text { training and rest phases, } \\
\text { and included a diary-type } \\
\text { weekly plan }\end{array}$ & $\begin{array}{l}\text { Primary outcome } \\
\text { Average change in pain } \\
\text { intensity VAS }(0-10) \text { at } \\
2 \text { months } \\
\text { Secondary outcomes } \\
\text { Constant-Murley score, } \\
\text { EQ-5D, shoulder ROM, } \\
\text { clinical signs of } \\
\text { impingement, strength of } \\
\text { abduction/adduction and } \\
\text { rotation }\end{array}$ & $\begin{array}{l}\text { Usual } \\
\text { physiotherapy, } \\
n=22 ; \text { HEP, } \\
n=16\end{array}$ & $\begin{array}{l}\text { Pain VAS } \\
\text { Baseline: HEP mean } 5.2(2.9 \\
\text { SD), physiotherapy } 5.0 \text { (1.4) } \\
2 \text { months: HEP mean } 3.5 \\
\text { (SD 2.3), physiotherapy } 3.8 \\
\text { (SD 2.6) } \\
\text { Constant-Murley score } \\
\text { Baseline: HEP 63.6 } \\
\text { (SD 14.7), physiotherapy } \\
60.1 \text { (SD 18.9) } \\
2 \text { months: HEP 75.5 } \\
\text { (SD 17.8), physiotherapy } \\
73.8 \text { (SD 19.1) } \\
\text { EQ-5D index } \\
\text { Baseline: HEP 0.907 } \\
\text { (SD 0.047), physiotherapy } \\
0.885 \text { (SD 0.049) } \\
2 \text { months: HEP 0.923 } \\
\text { (SD 0.037), physiotherapy } \\
0.933 \text { (SD 0.056) } \\
\text { EQ-5D VAS } \\
\text { Baseline: HEP 53.8 (SD 19), } \\
\text { physiotherapy 58.6 (SD 21.8) } \\
2 \text { months: HEP 71.6 } \\
\text { (SD 14.3), physiotherapy } \\
61.8 \text { (SD 19.6) }\end{array}$ & $\begin{array}{l}\text { Home-based therapy } \\
\text { programme on the basis } \\
\text { of an illustrated booklet } \\
\text { with independent } \\
\text { exercises twice a day is } \\
\text { comparable to formal } \\
\text { occupational therapy } \\
\text { (physiotherapy) in the } \\
\text { conservative treatment } \\
\text { of rotator cuff tears }\end{array}$ \\
\hline
\end{tabular}




\begin{tabular}{|c|c|c|c|c|c|c|c|c|}
\hline Study & Design/follow-up & Participants & Intervention & Comparator & Outcomes & Number analysed & Results & Conclusion \\
\hline $\begin{array}{l}\text { Granviken } \\
\text { and Vasseljen } \\
(2015)^{111}\end{array}$ & $\begin{array}{l}\text { Parallel } \\
\text { Two arms } \\
\text { Follow-up: } \\
6 \text { weeks and } \\
26 \text { weeks }\end{array}$ & $\begin{array}{l}\text { Forty-six participants } \\
\text { with subacromial } \\
\text { impingement }(n=23 \\
\text { in each group); } 22 \\
\text { females: } 24 \text { males; mean } \\
\text { age } 47.9 \text { (9.9 SD) years }\end{array}$ & $\begin{array}{l}\text { Supervised exercise } \\
\text { Up to } 10 \text { supervised } \\
\text { exercise treatments in } \\
\text { addition to home } \\
\text { exercises for } 6 \text { weeks }\end{array}$ & $\begin{array}{l}\text { Unsupervised (home) } \\
\text { exercise group } \\
\\
\text { One supervised exercise } \\
\text { treatment followed by } \\
\text { exercises at home for } \\
6 \text { weeks }\end{array}$ & $\begin{array}{l}\text { Primary outcome } \\
\text { SPADI [0 (best) to } \\
100 \text { (worse)] } \\
\text { Secondary outcomes } \\
\text { Average pain in the past } \\
\text { week (VAS 0-10), FABQ } \\
\text { physical activity scale } \\
\text { [0 (best) to } 24 \text { (worse)], } \\
\text { FABQ work scale [0 } \\
\text { (best) to } 42 \text { (worse)], } \\
\text { self-reported work } \\
\text { status, participant } \\
\text { satisfaction (patient } \\
\text { perceived treatment } \\
\text { benefit, seven-point } \\
\text { Likert scale; treatment } \\
\text { satisfaction, five-point } \\
\text { Likert scale; treatment), } \\
\text { shoulder AROM (flexion, } \\
\text { abduction, internal/ } \\
\text { external rotation) }\end{array}$ & $\begin{array}{l}26 \text { weeks: } \\
\text { supervised } \\
\text { exercise, } n=21 \text {; } \\
\text { unsupervised } \\
\text { (home) exercise } \\
\text { group, } n=18\end{array}$ & $\begin{array}{l}\text { SPADI } \\
\text { Baseline: home exercise } \\
\text { mean } 49 \text { (SD 12), supervised } \\
\text { exercise mean } 48 \text { (SD 19) } \\
6 \text { weeks: home exercise } \\
\text { mean } 32 \text { (SD 15), supervised } \\
\text { exercise mean } 32 \text { (SD 20) } \\
\text { 26 weeks: home exercise } \\
\text { mean 24 (SD 24), supervised } \\
\text { exercise mean 21 (SD18) } \\
\text { Average pain VAS } \\
\text { Baseline: home exercise } \\
\text { mean 6.3 (SD 1.3), } \\
\text { supervised exercise mean } \\
5.9 \text { (SD 2.2) } \\
6 \text { weeks: home exercise mean } \\
4.3 \text { (SD 2.2), supervised } \\
\text { exercise mean 4.1 (SD 2.1) } \\
\text { FABQ physical activity } \\
\text { Baseline: home exercise } \\
\text { mean 14 (SD 4), supervised } \\
\text { exercise mean 14.4 (SD 5) } \\
6 \text { weeks: home exercise } \\
\text { mean 10.6 (SD 5.3), } \\
\text { supervised exercise mean } \\
12.8 \text { (SD 5.8) } \\
\text { FABQ work } \\
\text { Baseline: home exercise } \\
\text { mean 20.6 (SD 7.1), } \\
\text { supervised exercise mean } \\
19.3 \text { (SD 12.4) } \\
6 \text { weeks: home exercise } \\
\text { mean 17.4 (SD 7.6), } \\
\text { supervised exercise mean } \\
16.2 \text { (SD 13.1) }\end{array}$ & $\begin{array}{l}\text { Supervision of more than } \\
\text { the first session of a } \\
\text { 6-week exercise regimen } \\
\text { did not cause significant } \\
\text { differences in pain and } \\
\text { disability in people with } \\
\text { subacromial impingement } \\
\text { No differences were } \\
\text { found in the primary } \\
\text { outcome, the SPADI. } \\
\text { Furthermore, no } \\
\text { differences were found } \\
\text { in the secondary } \\
\text { outcomes of pain, the } \\
\text { FABQ (physical activity } \\
\text { and work), participant } \\
\text { satisfaction or active } \\
\text { range of motion after } \\
\text { the intervention period }\end{array}$ \\
\hline
\end{tabular}


TABLE 58 Summary of trials comparing the effects of supervised vs. unsupervised exercise interventions (continued)

Work status (on sick leave)

6 weeks: home exercise 7/21, supervised exercise $10 / 23$

26 weeks: home exercise 4/18, supervised exercise $3 / 21$

No significant differences between groups for work status at 6 or 26 weeks

\section{Perceived benefit}

Much improved: home exercise $24 \%$, supervised exercise $52 \%$

Slightly improved: home exercise $57 \%$, supervised exercise $30 \%$

No change: home exercise $19 \%$, supervised exercise $9 \%$

Slightly worse: home exercise $0 \%$, supervised exercise $0 \%$

Much worse: home exercise $0 \%$, supervised exercise $9 \%$

\section{Treatment satisfaction}

Satisfied: home exercise $52 \%$, supervised exercise $83 \%$

Somewhat satisfied: home exercise $29 \%$, supervised exercise $4 \%$

Neither: home exercise 19\% supervised exercise $9 \%$ 


\begin{tabular}{|c|c|c|c|c|c|c|c|c|}
\hline Study & Design/follow-up & Participants & Intervention & Comparator & Outcomes & Number analysed & Results & Conclusion \\
\hline $\begin{array}{l}\text { Roddy et al. } \\
(2021)^{115}\end{array}$ & $\begin{array}{l}\text { Factorial } \\
2 \times 2 \text { arm } \\
\text { Follow-up: } \\
6 \text { weeks, } \\
6 \text { months and } \\
12 \text { months }\end{array}$ & $\begin{array}{l}\text { A total of } 256 \text { patients } \\
\text { with subacromial } \\
\text { impingement randomised } \\
\text { ( } n=64 \text { to each } \\
\text { treatment group, i.e. } 128 \\
\text { to each exercise group); } \\
52 \% \text { female: } 48 \% \text { male; } \\
\text { mean age } 54 \text { years; } \\
\text { mean SPADI score } 61 \\
\text { (SD 18) }\end{array}$ & $\begin{array}{l}\text { (1) Ultrasound-guided } \\
\text { injection and physiotherapist- } \\
\text { led exercise, or (3) unguided } \\
\text { injection and physiotherapist- } \\
\text { led exercise (supervised) } \\
\text { The exercise programme } \\
\text { comprised (1) scapular } \\
\text { stability and active } \\
\text { exercise with no } \\
\text { resistance, (2) range of } \\
\text { motion exercise with } \\
\text { scapular control, } \\
\text { isometrics and stretches } \\
\text { and (3) through-range } \\
\text { resistance exercise. } \\
\text { Six to eight sessions } \\
\text { over } 12 \text { weeks plus daily } \\
\text { home exercise: stage } 1 \text {, } \\
\text { hourly; stage 2, three or } \\
\text { four times per day; and } \\
\text { stage } 3 \text {, three times } \\
\text { per week }\end{array}$ & $\begin{array}{l}\text { (2) Ultrasound-guided } \\
\text { injection and exercise } \\
\text { leaflet, or (4) unguided } \\
\text { injection and exercise } \\
\text { leaflet (unsupervised) } \\
\text { Leaflet includes } \\
\text { information about } \\
\text { shoulder anatomy and } \\
\text { SIS, plus simple self-help } \\
\text { messages about pain } \\
\text { relief (including the } \\
\text { application of cold packs) } \\
\text { and activities. It includes } \\
\text { a small number of } \\
\text { standardised exercises, } \\
\text { including specific muscle } \\
\text { strengthening and ROM } \\
\text { exercises. Exercises are } \\
\text { not individualised, } \\
\text { supervised or progressed } \\
\text { by physiotherapists }\end{array}$ & $\begin{array}{l}\text { Primary outcome measure } \\
\text { SPADI at } 6 \text { months for } \\
\text { exercise interventions } \\
\text { Secondary outcomes } \\
\text { Current pain intensity } \\
\text { VAS, overall rating of } \\
\text { change, pain self-efficacy, } \\
\text { fear of movement, } \\
\text { physical health, exercise } \\
\text { adherence and patient } \\
\text { satisfaction }\end{array}$ & $\begin{array}{l}6 \text { weeks: } 94 \% \\
6 \text { months: } 88 \% \\
12 \text { months: } 80 \%\end{array}$ & $\begin{array}{l}\text { Physiotherapist-led exercise vs. } \\
\text { exercise leaflet } \\
\text { SPADI } \\
\text { Superior pain and function } \\
\text { scores for physiotherapist- } \\
\text { led exercise than exercise } \\
\text { leaflet at } 6 \text { months (adjusted } \\
\text { mean SPADI difference } \\
\text { between groups -8.23, } \\
95 \% \mathrm{CI}-14.14 \text { to }-2.32 \text {; } \\
p<0.001 \text { ), but not at } \\
6 \text { weeks or } 12 \text { months } \\
\text { Treatment satisfaction } \\
\text { More satisfaction with } \\
\text { treatment for } \\
\text { physiotherapist-led exercise } \\
\text { than exercise leaflet at } \\
6 \text { weeks (odds ratio } 15.81 ; \\
p<0.001 \text { ) and } 6 \text { months } \\
\text { (odds ratio } 8.86 ; p<0.001 \text { ) } \\
\text { Exercise adherence } \\
\text { Greater adherence to } \\
\text { physiotherapist-led exercise } \\
\text { than exercise leaflet at } \\
6 \text { weeks (odds ratio } 7.56 \text {; } \\
p=0.001 \text { ) and } 6 \text { months } \\
\text { (odds ratio } 6.09: p=0.002 \text { ) }\end{array}$ & $\begin{array}{l}\text { Physiotherapist-led } \\
\text { exercise for patients } \\
\text { with SIS leads to greater } \\
\text { improvements in pain } \\
\text { and function at } 6 \text { months, } \\
\text { but not at } 12 \text { months, } \\
\text { compared with providing } \\
\text { a standardised advice } \\
\text { and exercise leaflet }\end{array}$ \\
\hline
\end{tabular}


TABLE 58 Summary of trials comparing the effects of supervised vs. unsupervised exercise interventions (continued)

\begin{tabular}{|c|c|c|c|c|c|c|c|c|}
\hline Study & Design/follow-up & Participants & Intervention & Comparator & Outcomes & Number analysed & Results & Conclusion \\
\hline $\begin{array}{l}\text { Littlewood } \\
\text { et al. }(2016)^{67}\end{array}$ & $\begin{array}{l}\text { Parallel } \\
\text { Two arms } \\
\text { Follow-up: } \\
3 \text { months, } \\
6 \text { months and } \\
12 \text { months }\end{array}$ & $\begin{array}{l}\text { Eighty-six patients with } \\
\text { rotator cuff tendinopathy } \\
\text { randomised (usual } \\
\text { physiotherapy, } n=44 \text {; } \\
\text { self-management single } \\
\text { exercise programme, } \\
n=42 \text { ); } 50 \% \text { female; } \\
\text { mean age } 55 \text { years; } \\
\text { mean SPADI score } 49\end{array}$ & $\begin{array}{l}\text { Usual physiotherapy } \\
\text { Involved a range of } \\
\text { interventions, including } \\
\text { advice, stretching, } \\
\text { exercise, manual therapy, } \\
\text { massage, strapping, } \\
\text { acupuncture, } \\
\text { electrotherapy and } \\
\text { corticosteroid injection } \\
\text { at discretion of the } \\
\text { treating physiotherapist }\end{array}$ & $\begin{array}{l}\text { Self-management single } \\
\text { exercise programme } \\
\text { A single exercise } \\
\text { prescribed by the } \\
\text { physiotherapist within } \\
\text { the context of a self- } \\
\text { managed framework. } \\
\text { Affected shoulder } \\
\text { exercised against } \\
\text { gravity, with resistive } \\
\text { therapeutic band or } \\
\text { hand weight over three } \\
\text { sets of } 10-15 \text { repetitions } \\
\text { twice per day. Typically, } \\
\text { the exercise programme } \\
\text { might commence with } \\
\text { isometric abduction and } \\
\text { progress to isotonic } \\
\text { abduction. Exercise also } \\
\text { progressed through } \\
\text { increased repetitions } \\
\text { and load }\end{array}$ & $\begin{array}{l}\text { Primary outcome measure } \\
\text { SPADI at } 3 \text { months } \\
\text { Secondary outcomes } \\
\text { SPADI at } 6 \text { and } 12 \text { months, } \\
\text { health-related quality of } \\
\text { life (SF-36) }\end{array}$ & $\begin{array}{l}3 \text { months: } n=33 \\
\text { physiotherapy; } \\
n=27 \text { self- } \\
\text { management } \\
\text { single exercise } \\
\text { programme } \\
6 \text { months: } n=25 \\
\text { physiotherapy; } \\
n=23 \text { self- } \\
\text { management } \\
\text { single exercise } \\
\text { programme } \\
12 \text { months: } n=22 \\
\text { physiotherapy; } \\
n=20 \text { self- } \\
\text { management } \\
\text { single exercise } \\
\text { programme }\end{array}$ & $\begin{array}{l}\text { SPADI } \\
\text { Mean SPADI score at } \\
3 \text { months } 32.4 \text { (SD 20.2) for } \\
\text { the self-managed group, } \\
\text { 30.7 (SD 19.7) for usual } \\
\text { physiotherapy treatment } \\
\text { group; adjusted MD } 3.2 \\
\text { (95\% } \mathrm{Cl}-6.0 \text { to } 12.4 ; \\
p=0.49) \\
\text { Adjusted } \mathrm{MD} \text { at } 6 \text { months: } \\
-6.2(95 \% \mathrm{Cl}-16.1 \text { to } 3.6) \\
\text { Adjusted } \mathrm{MD} \text { at } 12 \text { months: } \\
-6.0(95 \% \mathrm{Cl}-19.7 \text { to } 7.6 \text { ) }\end{array}$ & $\begin{array}{l}\text { For the primary outcome } \\
\text { (i.e. the mean SPADI } \\
\text { score at } 3 \text { months), } \\
\text { there was no difference } \\
\text { between the self- } \\
\text { managed group and } \\
\text { usual physiotherapy } \\
\text { treatment group. At } 6 \\
\text { and } 12 \text { months, there } \\
\text { remained no significant } \\
\text { difference between } \\
\text { groups }\end{array}$ \\
\hline
\end{tabular}




\begin{tabular}{|c|c|c|c|c|c|c|}
\hline Study & Design/follow-up & Participants & Intervention & Comparator & Outcomes & Number analyse \\
\hline $\begin{array}{l}\text { Asensio- } \\
\text { García et al. } \\
(2018)^{112}\end{array}$ & $\begin{array}{l}\text { Parallel } \\
\text { Two arms } \\
\text { Follow-up: } \\
5 \text { weeks }\end{array}$ & $\begin{array}{l}\text { Seventy-four patients } \\
\text { randomised } \\
\text { (intervention, } n=36 ; \\
\text { control, } n=38 \text { ); } 58 \% \\
\text { females; mean age } 61 \\
\text { (10.9 SD) years }\end{array}$ & $\begin{array}{l}\text { Intervention group } \\
\text { Initial meeting with } \\
\text { physiotherapy, advice } \\
\text { about recommendations, } \\
\text { postural hygiene and } \\
\text { description of shoulder } \\
\text { exercises (with printed } \\
\text { guide) to perform at } \\
\text { home. Followed by } \\
\text { attendance at five } \\
\text { 1-hour, consecutive } \\
\text { group sessions } \\
\text { (over } 10 \text { days) with } \\
\text { physiotherapy for } \\
\text { performance/review of } \\
\text { exercise programme and } \\
\text { a subsequent session } 2 \\
\text { weeks later (supervised) }\end{array}$ & $\begin{array}{l}\text { Control group } \\
\text { Same initial and } \\
\text { subsequent session as } \\
\text { intervention group, but } \\
\text { no attendance at five } \\
\text { group sessions. In } \\
\text { interim, exercises to be } \\
\text { performed at home only } \\
\text { (unsupervised) }\end{array}$ & $\begin{array}{l}\text { Follow-up at } 5 \text { weeks } \\
\text { only: shoulder pain VAS } \\
\text { (0-10), Constant-Murley } \\
\text { scale [pain, ADL, ROM, } \\
\text { strength; O (worse) } \\
\text { to } 100 \text { (better)], } \\
\text { QuickDASH scale } \\
\text { (30 questions) }\end{array}$ & $\begin{array}{l}n=36 \\
\text { intervention; } \\
n=25 \text { control }\end{array}$ \\
\hline
\end{tabular}

Difference between means reduction in functional

$-0.1(95 \% \mathrm{Cl}-1.0$ to $0.7 ; \quad$ limitations (QuickDASH).

There were no differences,

Constant-Murley scores

Intervention group, mean

between the two groups

change 3.0 (1.4 SD)

Control group, mean change

3.1 (1.8 SD)

Constant-Murley scale

Difference between means

4.1 (95\% Cl -8.8 to 0.6;

$p=0.085$ )

Intervention group, mean

change -15.7 (SD 6.8)

Control group, mean change

-11.6 (SD 11.6)

QuickDASH scale

Difference between means

14.7 (95\% Cl 7.7 to 21.7

$p<0.001)$

Intervention group, mean

change 26.3 (SD 14.3)

Control group, mean change

11.6 (SD 11.9) 
TABLE 58 Summary of trials comparing the effects of supervised vs. unsupervised exercise interventions (continued)

\begin{tabular}{|c|c|c|c|c|c|c|c|c|}
\hline Study & Design/follow-up & Participants & Intervention & Comparator & Outcomes & Number analysed & Results & Conclusion \\
\hline $\begin{array}{l}\text { Contreras } \\
\text { et al. }(2018)^{113}\end{array}$ & $\begin{array}{l}\text { Parallel } \\
\text { Two arms } \\
\text { Follow-up: } \\
6 \text { weeks, } \\
12 \text { weeks and } \\
24 \text { weeks }\end{array}$ & $\begin{array}{l}\text { A total of } 271 \text { patients } \\
\text { were randomised } \\
\text { (standard physiotherapy, } \\
n=133 ; \text { self-administered, } \\
n=138) ; 75 \% \text { female : } \\
25 \% \text { male; mean age } 56 \\
\text { (12 SD) years }\end{array}$ & $\begin{array}{l}\text { Standard physiotherapy } \\
\text { Ten exercise sessions in } \\
\text { 6-week blocks (i.e. } \\
\text { minimum of } 10 \text { sessions } \\
\text { and up to a maximum of } \\
40 \text { sessions over } \\
24 \text { weeks, depending } \\
\text { on improvement) } \\
\text { (supervised) }\end{array}$ & $\begin{array}{l}\text { Self-administered } \\
\text { Rehabilitation provided } \\
\text { with home exercise } \\
\text { instruction manual and } \\
\text { DVD. Exercises included } \\
\text { daily ROM (postural, } \\
\text { active assisted, active } \\
\text { training of scapular } \\
\text { muscles), daily flexibility } \\
\text { (anterior and posterior } \\
\text { shoulder stretches) and } \\
\text { three strength exercises } \\
\text { per week (scapular, } \\
\text { rotator cuff) with } \\
\text { resistance bands (green). } \\
\text { Included advice } \\
\text { regarding analgesics and } \\
\text { pain relief (unsupervised) }\end{array}$ & $\begin{array}{l}\text { Primary outcome } \\
\text { Patient-perceived } \\
\text { recovery at } 6 \text { weeks } \\
\text { Secondary outcomes } \\
\text { Patient-perceived } \\
\text { recovery at } 12 \text { and } \\
24 \text { weeks: shoulder } \\
\text { pain VAS (0-10), } \\
\text { Constant-Murley scale } \\
\text { [pain, ADLs, ROM, } \\
\text { strength; } 0 \text { (worse) to } \\
100 \text { (better)], SF-36 } \\
\text { mental and physical } \\
\text { scales, simple shoulder } \\
\text { test, DASH score and } \\
\text { work status at } 6,12 \text { and } \\
24 \text { weeks }\end{array}$ & $\begin{array}{l}\text { Numbers not } \\
\text { given }\end{array}$ & $\begin{array}{l}\text { Pain VAS } \\
\text { Baseline: unsupervised mean } \\
7.2 \text { (SD 1.8), supervised } \\
\text { mean } 7.4 \text { (SD 2) } \\
6 \text { weeks: unsupervised mean } \\
5.3 \text { (SD 2.7), supervised } \\
\text { mean 4.6 (SD 2.9) } \\
12 \text { weeks: unsupervised } \\
\text { mean } 4.7 \text { (SD 2.9), } \\
\text { supervised mean 4.3 (SD 3.1) } \\
24 \text { weeks: unsupervised } \\
\text { mean } 4.9 \text { (SD 3.4), } \\
\text { supervised mean 4.5 (SD 3.0) } \\
\text { Constant-Murley scale } \\
\text { Baseline: unsupervised mean } \\
63.2 \text { (SD 15.2), supervised } \\
\text { mean 60.9 (SD 17.2) } \\
6 \text { weeks: unsupervised mean } \\
70.9 \text { (SD 18.5), supervised } \\
\text { mean 71.8 (SD 19.1) } \\
12 \text { weeks: unsupervised } \\
\text { mean 71.6 (SD 18.3), } \\
\text { supervised mean 68.2 } \\
\text { (SD 18.9) }\end{array}$ & $\begin{array}{l}\text { The self-administered } \\
\text { rehabilitation } \\
\text { programme compared } \\
\text { with standard } \\
\text { physiotherapy in adults } \\
\text { with a painful shoulder is } \\
\text { no different with regard } \\
\text { to function, quality of } \\
\text { life or disability in short } \\
\text { and medium term. } \\
\text { Similarly, it is not worse } \\
\text { with regard to patient } \\
\text { perceived recovery in } \\
\text { the short term, but in } \\
\text { the medium term (i.e. } \\
24 \text { weeks) there was an } \\
\text { increase in patient } \\
\text { perceived recovery by } \\
\text { those treated with } \\
\text { standard physiotherapy }\end{array}$ \\
\hline
\end{tabular}




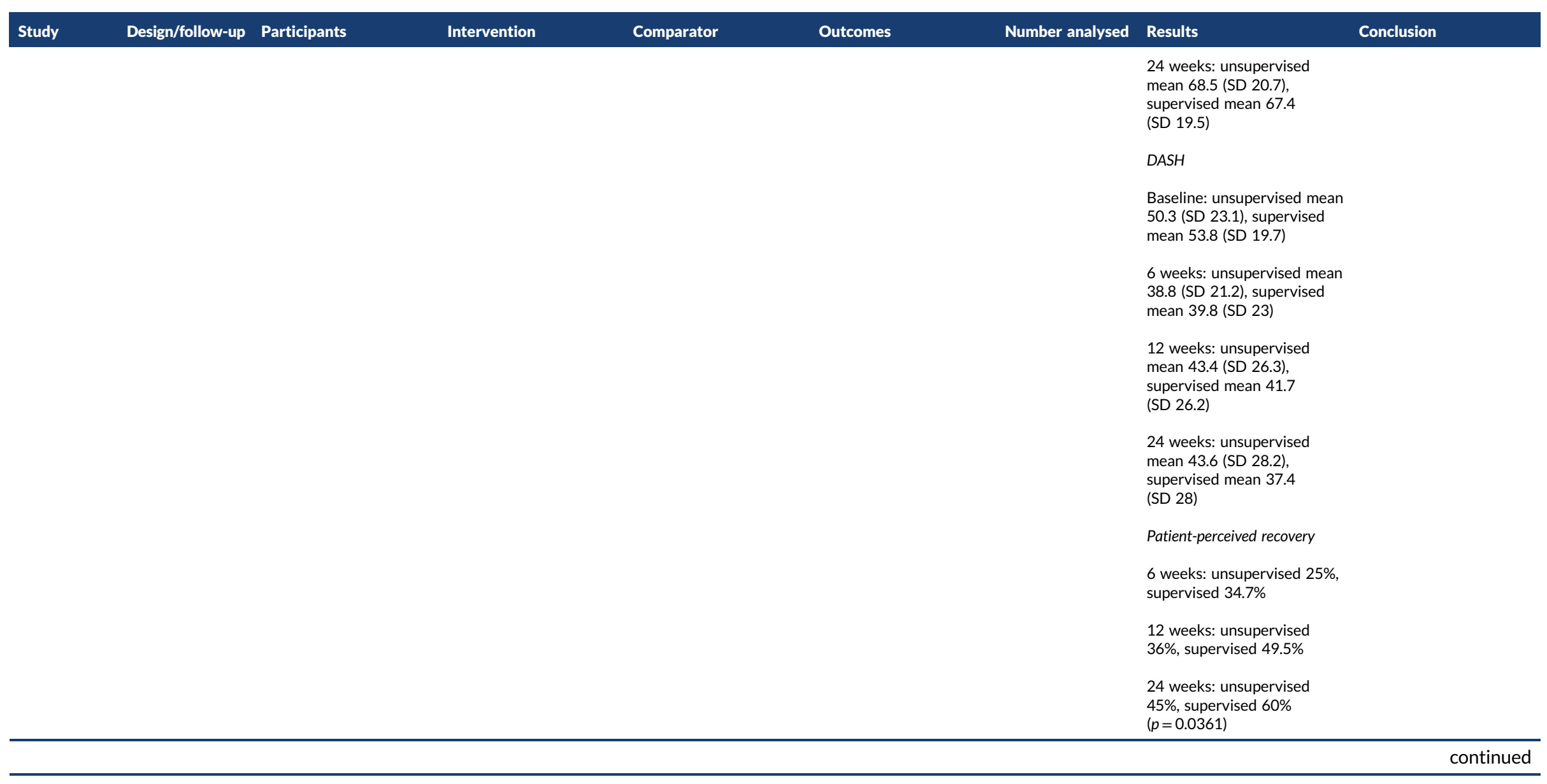


TABLE 58 Summary of trials comparing the effects of supervised vs. unsupervised exercise interventions (continued)

\begin{tabular}{|c|c|c|c|c|c|c|c|c|}
\hline Study & Design/follow-up & Participants & Intervention & Comparator & Outcomes & Number analysed & Results & Conclusion \\
\hline $\begin{array}{l}\text { Türkmen et al. } \\
(2020)^{114}\end{array}$ & $\begin{array}{l}\text { Parallel } \\
\text { Two arms } \\
\text { Follow-up: } \\
6 \text { weeks }\end{array}$ & $\begin{array}{l}\text { Thirty-three patients } \\
\text { randomised (VBR, } n=16 \text {; } \\
\text { physiotherapy, } n=17) ; \\
10 \text { females : } 20 \text { males; } \\
\text { mean age } 51 \text { ( } 8.54 \text { SD) } \\
\text { years of analysed } \\
\text { participants only }\end{array}$ & $\begin{array}{l}\text { Physiotherapist-supervised } \\
\text { rehabilitation group } \\
\text { Two exercise sessions } \\
\text { per week with } \\
\text { physiotherapist as well } \\
\text { as performing same } \\
\text { exercises as VBR } \\
\text { group three sets of } \\
10 \text { repetitions per day } \\
\text { at home for } 6 \text { weeks } \\
\text { (supervised) }\end{array}$ & $\begin{array}{l}\text { VBR group } \\
\text { A video rehabilitation } \\
\text { programme was } \\
\text { recorded by the } \\
\text { physiotherapist with the } \\
\text { participation of a patient. } \\
\text { Exercises performed three } \\
\text { sets of } 10 \text { repetitions per } \\
\text { day for } 6 \text { weeks. Patients } \\
\text { attended two sessions } \\
\text { with the physiotherapist } \\
\text { during the } 6 \text {-week period } \\
\text { to observe 'condition of } \\
\text { the patients, accuracy } \\
\text { of the exercises given, } \\
\text { and the clarity of the } \\
\text { commands..but no } \\
\text { feedback was given to } \\
\text { not interfere with the } \\
\text { effectiveness of the } \\
\text { program'114 (unsupervised) }\end{array}$ & $\begin{array}{l}\text { Rest, activity and night } \\
\text { pain VAS (O-10); } \\
\text { DASH; ASES shoulder } \\
\text { evaluation; SF-12 mental } \\
\text { and physical scales } \\
\text { [0 (worst) to } 100 \text { (best)]; } \\
\text { participant global rating } \\
\text { of change [one (worst) to } \\
\text { five (best) on a Likert } \\
\text { scale]; shoulder ROM }\end{array}$ & $\begin{array}{l}30 \text { patients } \\
\text { analysed ( } n=15 \text { in } \\
\text { each group) }\end{array}$ & $\begin{array}{l}\text { Rest pain VAS } \\
\text { Baseline: VBR mean } 1.13 \\
\text { (SD 1.8), physiotherapy } \\
\text { mean 3.47 (SD 2.74) } \\
\text { 6 weeks: VBR mean 0 } \\
\text { (SD 0), physiotherapy mean } \\
\text { 0.13 (SD 0.35) } \\
\text { Activity pain VAS: } \\
\text { Baseline: VBR mean } 6.47 \\
\text { (SD 1.18), physiotherapy } \\
\text { mean 6.87 (SD 1.72) } \\
\text { 6 weeks: VBR mean } 1.47 \\
\text { (SD 0.99), physiotherapy } \\
\text { mean 1.27 (SD 0.79) } \\
\text { Night pain VAS } \\
\text { Baseline: VBR mean } 5.13 \\
\text { (SD 2.56), physiotherapy } \\
\text { mean 5.6 (SD 2.06) } \\
\text { 6 weeks: VBR mean } 0.27 \\
\text { (SD 0.79), physiotherapy } \\
\text { mean 0.07 (SD 0.25) } \\
\text { DASH } \\
\text { Baseline: VBR mean } 32.68 \\
\text { (SD 14.48), physiotherapy } \\
\text { mean 37.45 (SD 18.25) } \\
6 \text { weeks: VBR mean } 5.39 \\
\text { (SD 5.67), physiotherapy } \\
\text { mean 5.5 (SD 5.12) }\end{array}$ & $\begin{array}{l}\text { Both rehabilitation } \\
\text { programmes were } \\
\text { effective on shoulder } \\
\text { ROM, pain, functionality } \\
\text { and quality of life for the } \\
\text { conservative treatment } \\
\text { of partial rotator cuff } \\
\text { tears. In addition, there } \\
\text { is no statistically significant } \\
\text { difference between the } \\
\text { rehabilitation programmes }\end{array}$ \\
\hline
\end{tabular}


Baseline: VBR mean 64.51 (SD 11.47), physiotherapy mean 54.18 (SD 15.2)

6 weeks: VBR mean 95.3 (SD 4.51), physiotherapy mean 94.4 (SD 5.38)

SF-12 physical

Baseline: VBR 39.54

(SD 6.23), physiotherapy 38.72 (SD 4.77)

6 weeks: VBR 51.64 (SD 3.49), physiotherapy mean 52.04 (SD 3.61)

SF-12 mental

Baseline: VBR mean 33.2

Baseline: $V$,
(SD 4.79), (SD 4.79), physiotherapy

6 weeks: VBR mean 38.06

(SD 3.49)

(SD 3.49), physiotherapy

Global rating of change

Much better: VBR 10/15

(66.7\%), physiotherapy $9 / 15$

(60\%)

Better: VBR 5/15 (33.3\%),

physiotherapy 6/15 (40\%)

ADL, activities of daily living; AROM, active range of shoulder movement; ASES, American Shoulder and Elbow Surgeons Standardized Shoulder Assessment Form; DASH, Disabilities of the Arm, Shoulder and Hand;

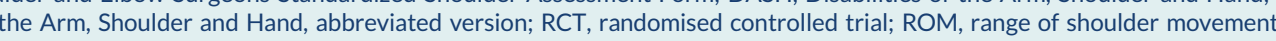

SF-12, Short Form questionnaire-12 items; SF-36, Short Form questionnaire-36 items; SIS, subacromial impingement symptoms: VAS, visual analogue Scale; VBR, video-based rehabilitation. 
TABLE 59 Studies comparing subacromial corticosteroid injection with no injection or placebo injection

\begin{tabular}{|c|c|c|c|c|c|c|c|}
\hline Study & Design/follow-up & Participants & Intervention & Comparator & Outcomes & Number analysed & Results/conclusions \\
\hline $\begin{array}{l}\text { Gialanella and } \\
\text { Prometti } \\
(2011)^{116}\end{array}$ & $\begin{array}{l}\text { Parallel } \\
\text { Three arms } \\
\text { Follow-up: } 3 \\
\text { and } 6 \text { months }\end{array}$ & $\begin{array}{l}\text { Mean age: one CSI - } \\
79 \text { years; no injection- } \\
79 \text { years; two CSIs - } \\
77 \text { years } \\
\text { Sex: } 92 \% \text { female } \\
\text { Population: rotator cuff } \\
\text { tears } \\
\text { DoS: } 5.4\end{array}$ & $\begin{array}{l}\text { (1) Unguided single } \\
\text { CSI }(n=20) \\
\text { Triamcinolone } \\
\text { acetonide }(40 \mathrm{mg})\end{array}$ & $\begin{array}{l}\text { (2) No injection }(n=20) \\
\text { No treatment } \\
\text { (3) Two unguided CSIs } \\
(n=20) \\
\text { Triamcinolone } \\
\text { acetonide }(40 \mathrm{mg}) \text { at } \\
\text { baseline and } 21 \text { days } \\
\text { later }\end{array}$ & $\begin{array}{l}\text { (1) Shoulder pain VAS } \\
\text { score } \\
\text { (2) Shoulder function: } \\
\text { Constant-Murley score }\end{array}$ & $\begin{array}{l}\text { CSI, } n=20 ; \text { no } \\
\text { CSI, } n=20\end{array}$ & $\begin{array}{l}\text { Intra-articular injection } \\
\text { of triamcinolone } \\
\text { improved pain relief at } \\
3 \text { months. There was } \\
\text { no difference between } \\
\text { groups in shoulder pain } \\
\text { at } 6 \text { months } \\
\text { There was no } \\
\text { difference between } \\
\text { groups in shoulder } \\
\text { function }\end{array}$ \\
\hline $\begin{array}{l}\text { Adebajo et al. } \\
(1990)^{117}\end{array}$ & $\begin{array}{l}\text { Parallel } \\
\text { Three arms } \\
\text { Follow-up: } \\
4 \text { weeks }\end{array}$ & $\begin{array}{l}\text { Mean age: CSI - } 51 \text { years; } \\
\text { placebo - } 55 \text { years; } \\
\text { NSAID - } 58 \text { years } \\
\text { Sex: } 47 \% \text { female } \\
\text { Population: rotator cuff } \\
\text { tendinitis } \\
\text { DoS: CSI = 8.6; } \\
\text { placebo = 8.5; } \\
\text { NSAID - } 7.7\end{array}$ & $\begin{array}{l}\text { Unguided CSI }(n=20) \\
1 \mathrm{ml} \text { of } 80 \mathrm{mg} / \mathrm{ml} \\
\text { triamcinolone with } \\
2 \mathrm{ml} \text { of } 0.5 \% \text { lignocaine } \\
\text { plus diclofenac- } \\
\text { matched placebo } \\
\text { tablets (one tablet } \\
\text { three times daily) }\end{array}$ & $\begin{array}{l}\text { (1) Placebo injection } \\
(n=20) \\
3 \mathrm{ml} \text { of } 0.5 \% \text { lignocaine } \\
\text { and placebo diclofenac } \\
\text { tablets (one tablet } \\
\text { three times daily) } \\
\text { (2) Oral diclofenac } \\
(n=20) \\
50-\mathrm{mg} \text { oral diclofenac } \\
\text { tablets three times } \\
\text { daily and subacromial } \\
\text { injection of } 3 \mathrm{ml} \text { of } \\
0.5 \% \text { lignocaine }\end{array}$ & $\begin{array}{l}\text { (1) Shoulder pain VAS } \\
\text { score }(0-10) \\
\text { (2) Shoulder function: } \\
\text { patient scale }(0-3)\end{array}$ & $\begin{array}{l}C S I, n=20 ; \\
\text { placebo, } n=20\end{array}$ & $\begin{array}{l}\text { Triamcinolone injection } \\
\text { improved shoulder pain } \\
\text { at } 4 \text { weeks compared } \\
\text { with placebo injection. } \\
\text { This difference was not } \\
\text { statistically significant }\end{array}$ \\
\hline $\begin{array}{l}\text { Alvarez et al. } \\
(2005)^{118}\end{array}$ & $\begin{array}{l}\text { Parallel } \\
\text { Two arms } \\
\text { Follow-up: } \\
2 \text { weeks, } 6 \text { weeks, } \\
3 \text { months and } \\
6 \text { months }\end{array}$ & $\begin{array}{l}\text { Mean age: CSI - } 50 \text { years; } \\
\text { placebo - } 46 \text { years } \\
\text { Sex: } 47 \% \text { female } \\
\text { Population: chronic } \\
\text { rotator cuff tendinosis } \\
\begin{array}{l}\text { DoS: CSI = } 3.8 \text { (years); } \\
\text { placebo }=2.5 \text { (years) }\end{array}\end{array}$ & $\begin{array}{l}\text { Unguided CSI }(n=31) \\
4 \mathrm{ml} \text { of } 2 \% \text { xylocaine } \\
\text { without adrenaline } \\
\text { combined with } \\
1 \mathrm{ml}(6 \mathrm{mg}) \text { of } \\
\text { betamethasone }\end{array}$ & 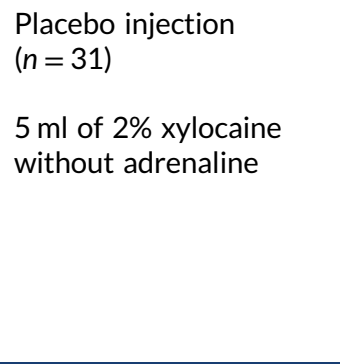 & $\begin{array}{l}\text { (1) Shoulder pain and } \\
\text { function (ASES score) } \\
\text { (2) Shoulder pain VAS } \\
\text { score (0-100) } \\
\text { (3) Disease-specific } \\
\text { quality of life (WORC) }\end{array}$ & $\begin{array}{l}\mathrm{CSI}, n=30 ; \\
\text { placebo, } n=28\end{array}$ & $\begin{array}{l}\text { No statistically } \\
\text { significant difference } \\
\text { between the two } \\
\text { groups for all outcomes } \\
\text { and time intervals }\end{array}$ \\
\hline
\end{tabular}




\begin{tabular}{|c|c|c|c|c|c|c|c|}
\hline Study & Design/follow-up & Participants & Intervention & Comparator & Outcomes & Number analysed & Results/conclusions \\
\hline $\begin{array}{l}\text { Alvarez- } \\
\text { Nemegyei } \\
\text { et al. }(2008)^{119}\end{array}$ & $\begin{array}{l}\text { Parallel } \\
\text { Two arms } \\
\text { Follow-up: } \\
6 \text { months }\end{array}$ & $\begin{array}{l}\text { Mean age: } 53 \text { years } \\
\text { Sex: } 77 \% \text { female } \\
\text { Population: subacromial } \\
\text { impingement syndrome } \\
\text { DoS: CSI = 8.1; } \\
\text { placebo = } 3.1\end{array}$ & $\begin{array}{l}\text { Unguided CSI }(n=27) \\
2 \mathrm{ml} \text { of } \\
\text { methylprednisolone } \\
\text { acetate suspension } \\
\text { plus } 1 \mathrm{ml} \text { of } \\
\text { lidocaine } 1 \%\end{array}$ & $\begin{array}{l}\text { Placebo injection } \\
(n=29) \\
3 \mathrm{ml} \text { of lidocaine } 1 \%\end{array}$ & $\begin{array}{l}\text { (1) Shoulder pain VAS } \\
\text { score }(0-100) \\
\text { (2) Shoulder function } \\
\text { (SDQ score) }\end{array}$ & $\begin{array}{l}\text { CSI, } n=15 \\
\text { placebo, } n=17\end{array}$ & $\begin{array}{l}\text { No differences were } \\
\text { detected between the } \\
\text { study groups in the } \\
\text { change in the range of } \\
\text { shoulder movement at } \\
3 \text { and } 6 \text { months of } \\
\text { follow-up. Subacromial } \\
\text { injection of a mixture } \\
\text { of methylprednisolone } \\
\text { plus lidocaine was not } \\
\text { more effective than } \\
\text { lidocaine only }\end{array}$ \\
\hline $\begin{array}{l}\text { Blair et al. } \\
(1996)^{120}\end{array}$ & $\begin{array}{l}\text { Parallel } \\
\text { Two arms } \\
\text { Follow-up: } \\
\text { CSI - } 33 \text { weeks; } \\
\text { placebo - } \\
28 \text { weeks }\end{array}$ & $\begin{array}{l}\text { Mean age: CSI - } 56 \text { years; } \\
\text { placebo - } 57 \text { years } \\
\text { Sex: } 80 \% \text { female } \\
\text { Population: subacromial } \\
\text { impingement syndrome } \\
\text { DoS: } 8 \text { (combined } \\
\text { average) }\end{array}$ & $\begin{array}{l}\text { Unguided CSI }(n=19) \\
2 \mathrm{ml} \text { of } 40 \mathrm{mg} / \mathrm{ml} \\
\text { triamcinolone } \\
\text { acetonide with } 4 \mathrm{ml} \\
\text { of } 1 \% \text { lidocaine } \\
\text { without adrenaline }\end{array}$ & $\begin{array}{l}\text { Placebo injection } \\
(n=21) \\
6 \mathrm{ml} \text { of } 1 \% \text { lidocaine } \\
\text { without adrenaline }\end{array}$ & $\begin{array}{l}\text { (1) Shoulder pain VAS } \\
\text { scale }(0-4) \\
\text { (2) Shoulder function: } \\
\text { clinician assessed }\end{array}$ & NR & $\begin{array}{l}\text { The mean pain score } \\
\text { after the injection was } \\
1.2 \text { points for the } \\
\text { corticosteroid group } \\
\text { and } 2.0 \text { points for the } \\
\text { control group. This } \\
\text { difference was significant } \\
(p<0.005) \text {. The use of } \\
\text { such injections can } \\
\text { substantially decrease } \\
\text { pain and increase the } \\
\text { range of motion of the } \\
\text { shoulder }\end{array}$ \\
\hline $\begin{array}{l}\text { Holt et al. } \\
(2013)^{7}\end{array}$ & $\begin{array}{l}\text { Parallel; external } \\
\text { pilot RCT } \\
\text { Two arms } \\
\text { Follow-up: } 4 \text { and } \\
12 \text { weeks }\end{array}$ & $\begin{array}{l}\text { Mean age: CSI - } 62 \text { years; } \\
\text { placebo - } 56 \text { years } \\
\text { Sex: } 65 \% \text { female } \\
\text { Population: rotator cuff } \\
\text { tendinopathy or } \\
\text { adhesive capsulitis } \\
\text { DoS: } \mathrm{CSI}=15.9 \\
\text { placebo }=10\end{array}$ & $\begin{array}{l}\text { Unguided CSI }(n=19) \\
40 \text { mg of } \\
\text { methylprednisone } \\
\text { acetate with lidocaine } \\
1 \% \text { in } 1 \mathrm{ml}\end{array}$ & $\begin{array}{l}\text { Placebo injection } \\
(n=21) \\
\text { Lidocaine } 1 \% \text { in } 1 \mathrm{ml}\end{array}$ & $\begin{array}{l}\text { (1) Shoulder function } \\
\text { (OSS) - secondary } \\
\text { outcome }\end{array}$ & & $\begin{array}{l}\text { This pilot trial showed } \\
\text { that it is feasible to } \\
\text { recruit participants } \\
\text { with shoulder pain in } \\
\text { the primary care } \\
\text { setting for a blinded, } \\
\text { randomised trial of } \\
\text { corticosteroid injection. } \\
\text { Clinical outcomes were } \\
\text { not analysed as part of } \\
\text { the pilot trial }\end{array}$ \\
\hline
\end{tabular}


TABLE 59 Studies comparing subacromial corticosteroid injection with no injection or placebo injection (continued)

\begin{tabular}{|c|c|c|c|c|c|c|c|}
\hline Study & Design/follow-up & Participants & Intervention & Comparator & Outcomes & Number analysed & Results/conclusions \\
\hline $\begin{array}{l}\text { Hong et al. } \\
(2011)^{121}\end{array}$ & $\begin{array}{l}\text { Parallel } \\
\text { Three arms } \\
\text { Follow-up: } \\
8 \text { weeks }\end{array}$ & $\begin{array}{l}\text { Mean age: CSI } 40 \mathrm{mg} \text { - } \\
51 \text { years; placebo - } \\
51 \text { years; CSI } 20 \mathrm{mg} \text { - } \\
49 \text { years } \\
\text { Sex: } 59 \% \text { female } \\
\text { Population: periarticular } \\
\text { shoulder disorders } \\
\text { DoS: } \mathrm{CSI} 40 \mathrm{mg}=8.9 ; \\
\text { placebo }=8.6 \text {; } \\
\text { CSI } 20 \mathrm{mg}=13\end{array}$ & $\begin{array}{l}\text { (1) Guided CSI } 40 \mathrm{mg} \\
(n=30) \\
4 \mathrm{ml} \text { of } 40 \mathrm{mg} \\
\text { triamcinolone } \\
\text { acetonide }\end{array}$ & $\begin{array}{l}\text { (2) Guided placebo } \\
(n=30) \\
4 \mathrm{ml} \text { of } 1 \% \text { lidocaine } \\
\text { (3) Guided CSI } 20 \mathrm{mg} \\
(n=30) \\
2 \mathrm{ml} \text { of } 20 \mathrm{mg} \\
\text { triamcinolone acetonide } \\
\text { and } 2 \mathrm{ml} \text { of } 1 \% \text { lidocaine }\end{array}$ & $\begin{array}{l}\text { (1) Shoulder pain VAS } \\
\text { score }(0-10) \\
\text { (2) Shoulder function } \\
\text { (SDQ) }\end{array}$ & $\begin{array}{l}\text { CSI } 40 \mathrm{mg}, n=27 \\
\text { placebo, } n=27\end{array}$ & $\begin{array}{l}\text { In groups } 1 \text { ( } 40 \mathrm{mg} \\
\text { of triamcinolone } \\
\text { acetonide) and } 2 \\
\text { ( } 20 \mathrm{mg} \text { of triamcinolone } \\
\text { acetonide) there were } \\
\text { significant differences } \\
\text { in mean changes post } \\
\text { treatment at weeks } 2,4 \\
\text { and } 8 \text { compared with } \\
\text { pre treatment (paired } \\
t \text {-test, all } p=0.001 \text { ), } \\
\text { whereas in group } 3 \\
\text { (placebo) there was no } \\
\text { significant difference post } \\
\text { treatment at weeks } 2,4 \\
\text { and } 8 \text { compared with pre } \\
\text { treatment (paired } t \text {-test, } \\
p=0.084, p=0.107 \text { and } \\
p=0.113, \text { respectively) }\end{array}$ \\
\hline $\begin{array}{l}\text { Penning et al. } \\
(2012)^{122}\end{array}$ & $\begin{array}{l}\text { Parallel } \\
\text { Three arms } \\
\text { Follow-up: 3, } 6 \\
\text { and } 12 \text { weeks }\end{array}$ & $\begin{array}{l}\text { Mean age: CSI - } 52 \text { years; } \\
\text { placebo - } 54 \text { years; HA - } \\
53 \text { years } \\
\text { Sex: } 53 \% \text { female } \\
\text { Population: subacromial } \\
\text { impingement syndrome } \\
\text { DoS: } 62 \% \text { of CSI group } \\
\text { mean > } 26 \text { weeks; } 71 \% \\
\text { of placebo group mean } \\
>26 \text { weeks }\end{array}$ & $\begin{array}{l}\text { (1) Unguided CSI } \\
(n=53) \\
2 \mathrm{ml} \text { of triamcinolone } \\
\text { acetonide } 10 \mathrm{mg} / \mathrm{ml} \\
\text { with } 8 \mathrm{ml} \text { of lidocaine } \\
1 \%\end{array}$ & $\begin{array}{l}\text { (2) Placebo injection } \\
(n=55) \\
2 \mathrm{ml} \text { of } \mathrm{NaCl} 0.9 \% \text { with } \\
8 \mathrm{ml} \text { of lidocaine } 1 \% \\
\text { (3) } \mathrm{HA} \text { injection }(n=51) \\
2 \mathrm{ml} \text { of } \mathrm{HA} \text { with } 8 \mathrm{ml} \text { of } \\
\text { lidocaine } 1 \%\end{array}$ & $\begin{array}{l}\text { (1) Shoulder pain } \\
\text { and function: } \\
\text { Constant-Murley } \\
\text { score } \\
\text { (2) Shoulder pain VAS } \\
\text { scale } \\
\text { (3) Shoulder function }\end{array}$ & $\begin{array}{l}\text { CSI, } n=45 \\
\text { placebo, } n=48\end{array}$ & $\begin{array}{l}\text { Compared with placebo } \\
\text { injections, corticosteroids } \\
\text { were significantly better } \\
\text { in terms of pain reduction, } \\
\text { but only in the short term } \\
\text { at } 6 \text { weeks }(p=0.006)\end{array}$ \\
\hline
\end{tabular}




\begin{tabular}{|c|c|c|c|c|c|c|c|}
\hline Study & Design/follow-up & Participants & Intervention & Comparator & Outcomes & Number analysed & Results/conclusions \\
\hline $\begin{array}{l}\text { Petri et al. } \\
(1987)^{123}\end{array}$ & $\begin{array}{l}\text { Parallel } \\
\text { Four arms } \\
\text { Follow-up: } \\
4 \text { weeks }\end{array}$ & $\begin{array}{l}\text { Mean age: NR } \\
\text { Sex: } 31 \% \text { female } \\
\text { Population: } \\
\text { supraspinatus } \\
\text { tendinitis } \pm \text { subacromial } \\
\text { bursitis } \\
\text { Dos: } 3.9\end{array}$ & $\begin{array}{l}\text { 1) Unguided CSI } \\
(n=25) \\
1 \mathrm{cc} \text { of } 40 \mathrm{mg} / \mathrm{ml} \\
\text { triamcinolone with } 3 \mathrm{cc} \\
\text { of } 1 \% \text { lidocaine plus } \\
\text { placebo naproxen pill } \\
\text { twice a day for } \\
30 \text { days }\end{array}$ & $\begin{array}{l}\text { (2) Placebo injection } \\
\text { ( } n=25 \text { ) } \\
4 \mathrm{cc} \text { of } 1 \% \text { lidocaine } \\
\text { plus placebo naproxen } \\
\text { pill two times per day } \\
\text { for } 30 \text { days } \\
\text { (3) Unguided CSI plus } \\
\text { NSAID ( } n=25 \text { ) } \\
1 \mathrm{cc} \text { of } 40 \mathrm{mg} / \mathrm{ml} \\
\text { triamcinolone with } 3 \mathrm{cc} \\
\text { of } 1 \% \text { lidocaine plus } \\
\text { naproxen pill (500 mg) } \\
\text { two times per day for } \\
30 \text { days } \\
\text { (4) Placebo injection } \\
\text { plus NSAID ( } n=25 \text { ) } \\
4 \text { cc of } 1 \% \text { lidocaine } \\
\text { plus naproxen (500 mg) } \\
\text { two times per day for } \\
30 \text { days }\end{array}$ & $\begin{array}{l}\text { (1) Shoulder pain } \\
\text { (VAS 0-5) } \\
\text { (2) Shoulder function } \\
\text { (clinician assessed) }\end{array}$ & $\begin{array}{l}\text { CSI, } n=25 \\
\text { placebo, } n=25\end{array}$ & $\begin{array}{l}\text { Both triamcinolone } \\
(p=0.00005) \text { and } \\
\text { naproxen }(p=0.02) \text { are } \\
\text { superior to placebo in } \\
\text { the treatment of the } \\
\text { painful shoulder }\end{array}$ \\
\hline
\end{tabular}


TABLE 59 Studies comparing subacromial corticosteroid injection with no injection or placebo injection (continued)

\begin{tabular}{|c|c|c|c|c|c|c|c|}
\hline Study & Design/follow-up & Participants & Intervention & Comparator & Outcomes & Number analysed & Results/conclusions \\
\hline $\begin{array}{l}\text { Vecchio et al. } \\
(1993)^{124}\end{array}$ & $\begin{array}{l}\text { Parallel } \\
\text { Two arms } \\
\text { Follow-up: } \\
12 \text { weeks }\end{array}$ & $\begin{array}{l}\text { Mean age: CSI - } 56 \text { years; } \\
\text { placebo - } 57 \text { years } \\
\text { Sex: } 60 \% \text { female } \\
\text { Population: acute } \\
\text { rotator cuff tendinitis } \\
\text { DoS: CSI = } 5 \text { weeks; } \\
\text { placebo = } 4 \text { weeks }\end{array}$ & $\begin{array}{l}\text { Unguided CSI }(n=29) \\
40 \mathrm{mg} \text { of } \\
\text { methylprednisolone } \\
\text { plus } 1 \mathrm{ml} \text { of } \\
\text { lignocaine } 1 \%\end{array}$ & 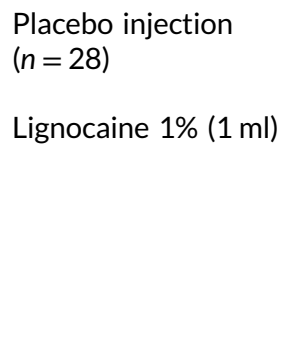 & (1) Shoulder pain & NR & $\begin{array}{l}\text { Although steroid- } \\
\text { treated patients seem } \\
\text { to have improved to a } \\
\text { greater extent than } \\
\text { lignocaine-treated } \\
\text { patients at } 2 \text { weeks, } \\
\text { this was not statistically } \\
\text { significant. This small } \\
\text { improvement was not } \\
\text { sustained by } 12 \text { weeks }\end{array}$ \\
\hline $\begin{array}{l}\text { Withrington } \\
\text { et al. }(1985)^{90}\end{array}$ & $\begin{array}{l}\text { Parallel } \\
\text { Two arms } \\
\text { Follow-up: } \\
8 \text { weeks }\end{array}$ & $\begin{array}{l}\text { Mean age: CSI - } 61 \text { years; } \\
\text { placebo - } 55 \text { years } \\
\text { Sex: } 76 \% \text { female } \\
\text { Population: } \\
\text { supraspinatus tendonitis } \\
\text { DoS: } \mathrm{CSI}=4.1 \text {; } \\
\text { placebo }=4.6\end{array}$ & $\begin{array}{l}\text { Unguided CSI }(n=12) \\
80 \mathrm{mg} \text { of } \\
\text { methylprednisolone } \\
\text { diluted in } 2 \mathrm{ml} \text { of } 2 \% \\
\text { lignocaine }\end{array}$ & $\begin{array}{l}\text { Placebo injection } \\
(n=13) \\
4 \mathrm{ml} \text { of saline }(0.9 \%)\end{array}$ & $\begin{array}{l}\text { (1) Shoulder pain VAS } \\
(0-10)\end{array}$ & $\begin{array}{l}\text { CSI, } n=12 ; \\
\text { placebo, } n=13\end{array}$ & $\begin{array}{l}\text { The VAS score in group } \\
1 \text { (steroid) improved } \\
\text { by a mean of } 2.72 \\
\text { between weeks } 0 \text { and } \\
8 \text {, whereas group } 2 \\
\text { (placebo) showed a } \\
\text { mean improvement } \\
\text { of } 1.16 \text { in the same } \\
\text { period. This difference } \\
\text { was not statistically } \\
\text { significant }(p>0.05)\end{array}$ \\
\hline
\end{tabular}




\section{MEDLINE search strategy}

Medline (Ovid MEDLINE ${ }^{\circledR}$ Epub Ahead of Print, In-Process \& Other Non-Indexed Citations, Ovid MEDLINE ${ }^{\circledR}$ Daily and Ovid MEDLINE ${ }^{\circledR}$ )

Date range searched: 1946 to present.

Date searched: June 2020.

\section{Search strategy}

1. Shoulder Pain/ (4378)

2. Shoulder Impingement Syndrome/ (1655)

3. Rotator Cuff/and Tendinopathy/ (477)

4. Shoulder/and Bursitis/or Tendinopathy/) (438)

5. ((shoulder* or rotator cuff or subacromial or sub-acromial) adj5 (bursitis or impinge* or tendinitis or tendonitis or tendinopathy or pain*)).ti,ab. $(12,483)$

6. 1 or 2 or 3 or 4 or $5(14,765)$

7. (Cortisone/or Glucocorticoids/) and Injections/ (1354)

8. ((subacromial or sub-acromial or corticosteroid* or cortisone or glucocorticoid*) adj5 inject*).ti,ab. (5093)

9. CSI.ti,ab. (3029)

10. 7 or 8 or $9(9227)$

11. 6 and 10 (480)

12. randomized controlled trial.pt. $(477,274)$

13. controlled clinical trial.pt. $(92,948)$

14. (randomized or randomised).ab. $(522,076)$

15. placebo.ab. $(195,900)$

16. drug therapy.fs. $(2,088,506)$

17. randomly.ab. $(306,731)$

18. trial.ab. $(455,979)$

19. groups.ab. $(1,887,811)$

20. 12 or 13 or 14 or 15 or 16 or 17 or 18 or $19(4,404,372)$

21. exp animals/not humans.sh. $(4,554,611)$

22. 20 not $21(3,810,278)$

23. 11 and 22 (320). 




\section{EME HS\&DR \\ HTA \\ PGFAR \\ PHR}

Part of the NIHR Journals Library

www.journalslibrary.nihr.ac.uk

This report presents independent research funded by the National Institute for Health Research (NIHR).

The views expressed are those of the author(s) and not necessarily those of the NHS, the NIHR or the

Department of Health and Social Care 